\title{
Synthesis, Electrochemistry and Optical Properties of Highly Conjugated Alkynyl-Ferrocenes and -Biferrocenes
}

Troy L. R. Bennett, ${ }^{a}$ Luke. A. Wilkinson, ${ }^{\text {, }}{ }^{*}$ Jasmine M. A. Lok, ${ }^{\mathrm{b}}$ Robert C. P. O’Toole, ${ }^{\mathrm{b}}$ Nicholas J. Long $^{\mathrm{a}, *}$

a Department of Chemistry, Imperial College London, MSRH, White City Campus, London, W12 0BZ, UK.

${ }^{\mathrm{b}}$ Department of Chemistry, University of York, Heslington, York, YO10 5DD, UK.

\section{Contents}

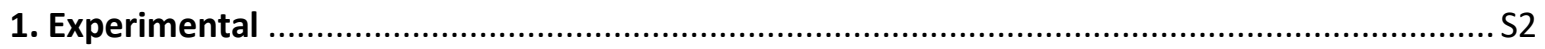

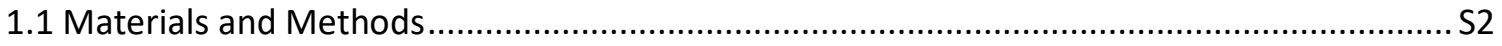

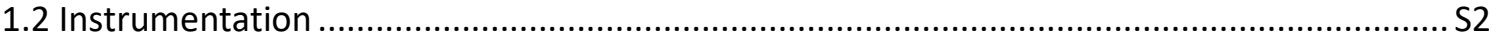

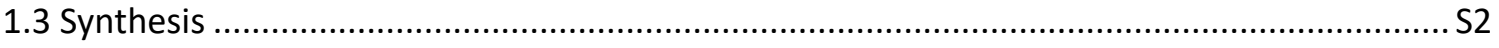

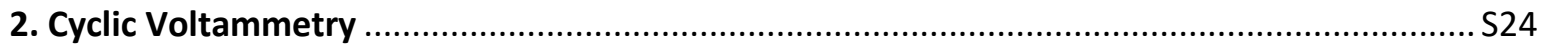

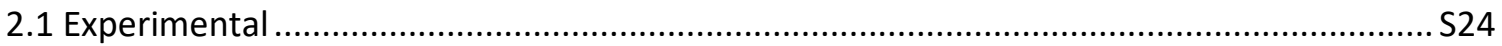

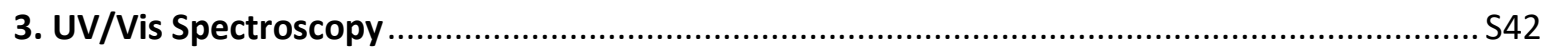

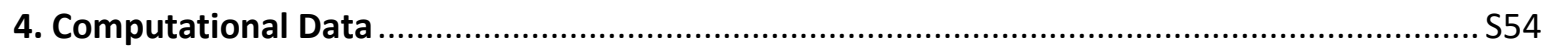

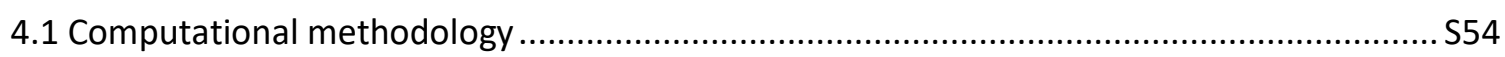

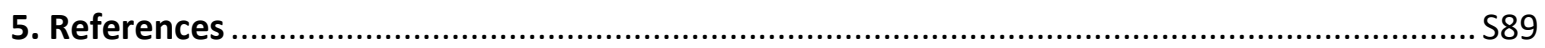




\section{Experimental}

\subsection{Materials and Methods}

$1,1^{\prime}$-Diiodoferrocene and 1,1'"-diiodobiferrocene were synthesised according to a previously published procedure. ${ }^{1}$ 4-Ethynylpyridine, 4-ethynylthioanisole, 3-ethynylpyridine and 3ethynylthioanisole were also synthesised as previously reported. ${ }^{2}$ All other reagents were obtained from commercial sources and used as received. Diisopropylamine was distilled, sparged with nitrogen and stored in an inert atmosphere over activated molecular sieves prior to use. All other reaction solvents were obtained from a Grubbs-type solvent purification system and were sparged with nitrogen prior to use. All reactions were performed under an inert atmosphere using standard Schlenk line techniques and work-ups were performed under air.

\subsection{Instrumentation}

${ }^{1} \mathrm{H}$ and ${ }^{13} \mathrm{C}\left\{{ }^{1} \mathrm{H}\right\}$ NMR spectra were recorded on a Bruker Avance 400 or $500 \mathrm{MHz}$ spectrometer and referenced to the residual solvent peaks of $\mathrm{CDCl}_{3}$ or $\mathrm{CD}_{2} \mathrm{Cl}_{2}$ at 7.26 or 5.32 and 77.2 or $54.0 \mathrm{ppm}$ respectively. ${ }^{13} \mathrm{C}\left\{{ }^{1} \mathrm{H}\right\}$ spectra were fully assigned where possible using $2 \mathrm{D}$ correlation spectroscopy. Coupling constants are measured in $\mathrm{Hz}$. Mass spectrometry analyses were conducted by Lisa Haigh of the Mass Spectrometry Service, Imperial College London. Microanalyses were carried out at the Science Centre, London Metropolitan University, by Stephen Boyer using a Thermo Scientific (Carlo Erba) Flash 2000 Organic Elemental Analyzer, configured for \%CHN. Infrared spectra were recorded on a PerkinElmer Spectrum FT-IR spectrometer. UV/Vis spectroscopy was recorded on an Agilent Cary60 spectrometer.

\subsection{Synthesis}

General procedure for the coupling of terminal alkynes to iodoferrocenes

- specific details regarding molar equivalents and column conditions are reported below.

This synthetic procedure is adapted from a published method. ${ }^{2}$ A Schlenk tube is charged with $1,1^{\prime}$ diiodoferrocene, terminal alkyne, $\mathrm{Cul}(5 \mathrm{~mol} \%)$ and $\mathrm{Pd}\left(\mathrm{P}^{\mathrm{t}} \mathrm{Bu}_{3}\right)_{2}(5 \mathrm{~mol} \%)$ then placed under an inert atmosphere. Anhydrous DIPA is added to the reaction vessel via cannula and the reaction is stirred overnight at room temperature to generate a light orange precipitate. Removal of the solvent in vacuo leads to a dark brown crude material which can be purified by column chromatography. 
1-lodo-1'-(4-(ethynyl)pyridine)ferrocene: (2a)

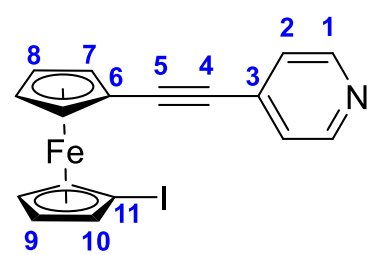

Following the general procedure, 1,1'-diiodoferrocene ( $9.00 \mathrm{~g}, 20.76 \mathrm{mmol})$, Cul $(0.03 \mathrm{~g}, 0.18 \mathrm{mmol})$, 4-ethynylpyridine $(0.36 \mathrm{~g}, 3.47 \mathrm{mmol})$ and $\mathrm{Pd}\left(\mathrm{P}^{\mathrm{t}} \mathrm{Bu}_{3}\right)_{2}(0.09 \mathrm{~g}, 0.18 \mathrm{mmol})$ gave a brown oil which was purified by chromatography on a silica column, eluted with $\mathrm{n}$-hexane/DCM $(1: 0 \rightarrow 4: 1 \mathrm{v} / \mathrm{v})$. The product presented as an orange solid (0.90 g, $2.19 \mathrm{mmol}, 63 \%)$.

${ }^{1} \mathrm{H} \mathrm{NMR}\left(\mathrm{CDCl}_{3}, 298 \mathrm{~K}, 400 \mathrm{MHz}\right): \delta_{\mathrm{H}}=8.57\left(\mathrm{dd},{ }^{3} \mathrm{~J}_{\mathrm{H}-\mathrm{H}}=4.4,{ }^{4} J_{\mathrm{H}-\mathrm{H}}=1.0 \mathrm{~Hz}, 2 \mathrm{H}, \mathrm{H2}\right), 7.36\left(\mathrm{dd},{ }^{3} \int_{\mathrm{H}-\mathrm{H}}=4.4\right.$, $\left.{ }^{4} \mathrm{~J}_{\mathrm{H}-\mathrm{H}}=1.0 \mathrm{~Hz}, 2 \mathrm{H}, H 1\right), 4.51\left(\mathrm{t},{ }^{3} \mathrm{~J}_{\mathrm{H}-\mathrm{H}}=2.0 \mathrm{~Hz}, 2 \mathrm{H}, H 8\right), 4.46\left(\mathrm{t},{ }^{3} \mathrm{~J}_{\mathrm{H}-\mathrm{H}}=2.0 \mathrm{~Hz}, 2 \mathrm{H}, H 9\right), 4.33\left(\mathrm{t},{ }^{3} \mathrm{~J}_{\mathrm{H}-\mathrm{H}}=2.0\right.$ $\mathrm{Hz}, 2 \mathrm{H}, H 7), 4.25\left(\mathrm{t},{ }^{3} \mathrm{~J}_{\mathrm{H}-\mathrm{H}}=2.0 \mathrm{~Hz}, 2 \mathrm{H}, H 10\right) \mathrm{ppm} ;{ }^{13} \mathrm{C}\left\{{ }^{1} \mathrm{H}\right\} \mathrm{NMR}(\mathrm{CDCl}, 298 \mathrm{~K}, 100 \mathrm{MHz}): \delta_{\mathrm{C}}=149.5(\mathrm{C} 1)$, 132.0 (C3), 125.4 (C2), 92.7 (C5), 84.5 (C4), 76.5 (C9), 74.4 (C10), 72.5 (C8), 70.9 (C7), 66.0 (C6), 41.3 (C11) ppm; IR: 2205 (-C $\equiv C-) \mathrm{cm}^{-1}$; MS ES+: calcd. for $\mathrm{C}_{17} \mathrm{H}_{13} \mathrm{FeNI}$ [M]+ 413.9442; found. 413.9456; EA calcd. C 49.44, H 2.93, N 3.39; found C 49.27, H 2.93, N 3.18.

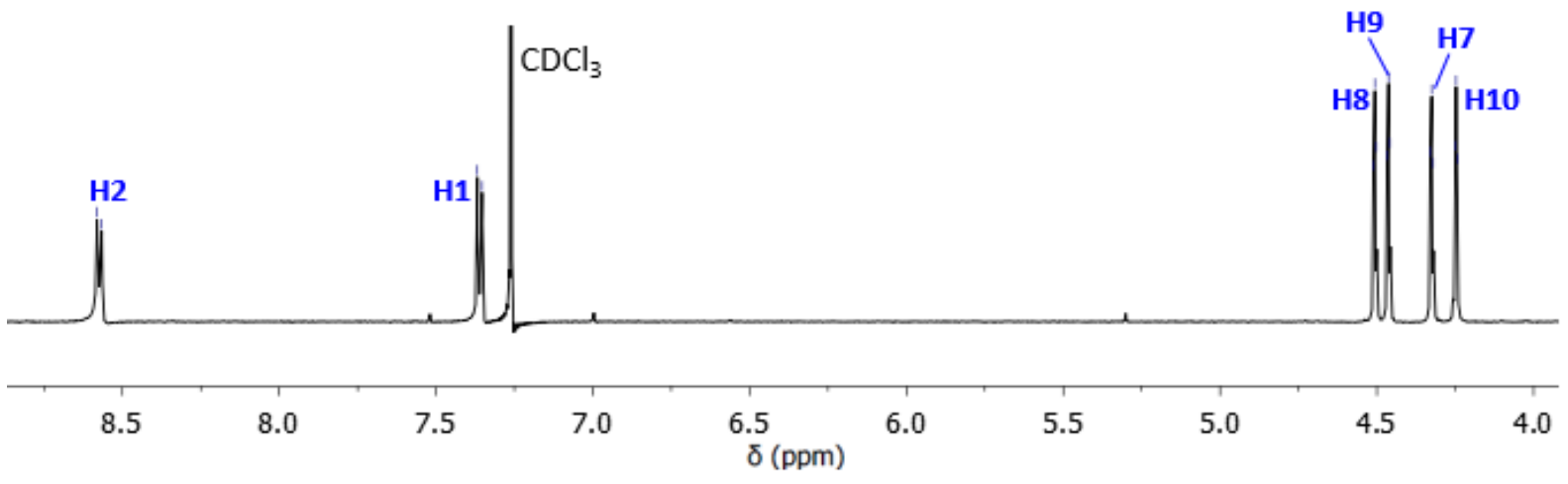

Figure S1: The ${ }^{1} \mathrm{H}$ NMR spectrum of $2 \mathrm{a}$ in $\mathrm{CDCl}_{3}$

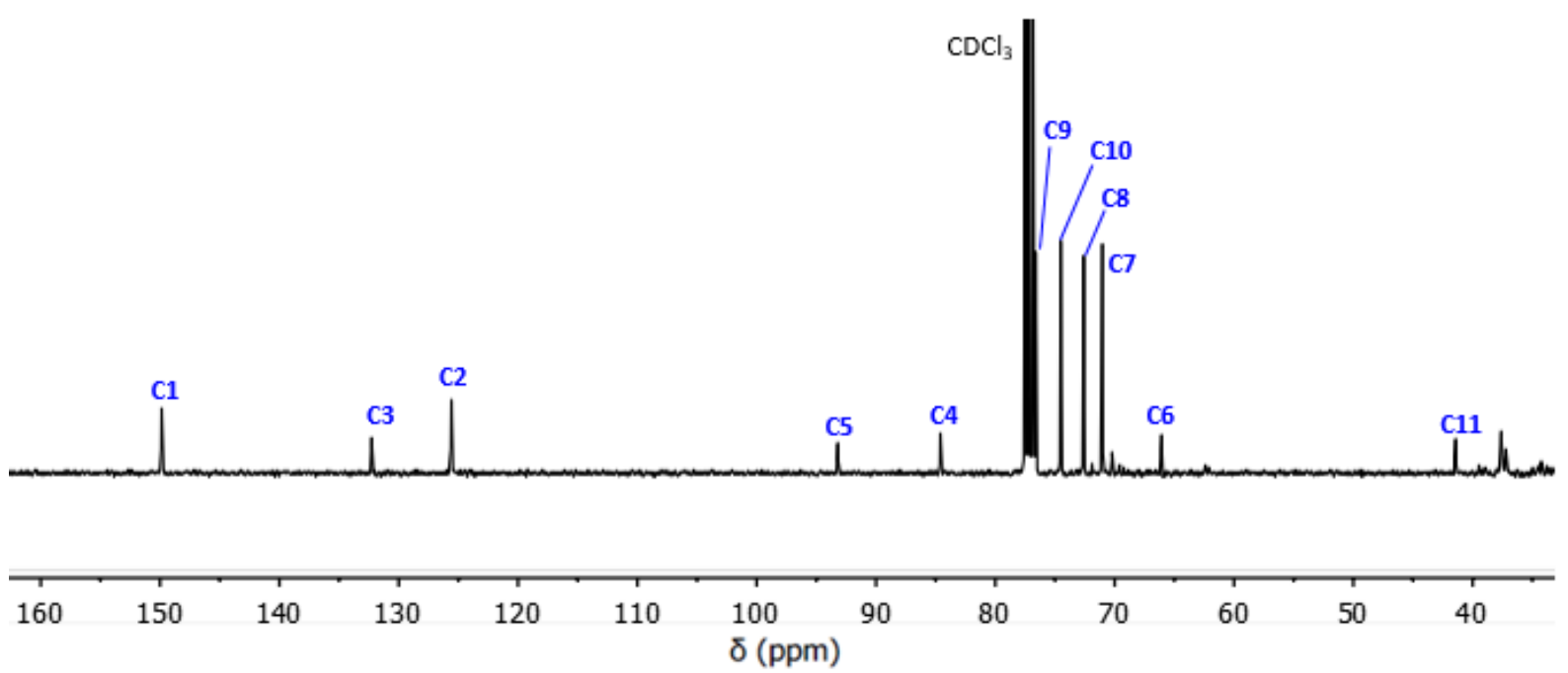

Figure S2: The ${ }^{13} \mathrm{C}\left\{{ }^{1} \mathrm{H}\right\}$ NMR spectrum of $\mathbf{2} \mathbf{a}$ in $\mathrm{CDCl}_{3}$ 
1-lodo-1'-(4-(ethynyl)thioanisole)ferrocene: $(2 \mathrm{c})^{2}$

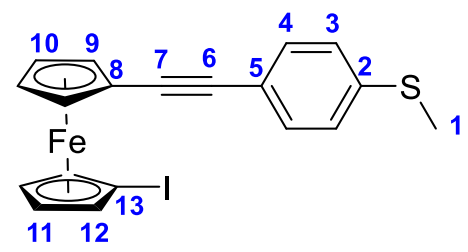

Following the general procedure, 1,1'-diiodoferrocene $(5.90 \mathrm{~g}, 13.48 \mathrm{mmol})$, Cul ( $0.03 \mathrm{~g}, 0.14 \mathrm{mmol})$, 4-ethynylthioanisole $(0.40 \mathrm{~g}, 2.70 \mathrm{mmol})$ and $\mathrm{Pd}\left(\mathrm{P}^{\mathrm{t}} \mathrm{Bu}_{3}\right)_{2}(0.07 \mathrm{~g}, 0.14 \mathrm{mmol})$ yielded a brown oil which was purified by chromatography on a silica column, eluted with $n$-hexane/DCM (1:0 $\rightarrow$ 4:1 v/v) giving the product as an orange solid ( $0.70 \mathrm{~g}, 1.16 \mathrm{mmol}, 43 \%)$.

${ }^{1} \mathrm{H}$ NMR $\left(\mathrm{CDCl}_{3}, 298 \mathrm{~K}, 400 \mathrm{MHz}\right): \delta_{\mathrm{H}}=7.42\left(\mathrm{dd},{ }^{3} \mathrm{~J}_{\mathrm{H}-\mathrm{H}}=6.8,{ }^{4} \mathrm{~J}_{\mathrm{H}-\mathrm{H}}=2.0 \mathrm{~Hz}, 2 \mathrm{H}, H 4\right), 7.19\left(\mathrm{dd},{ }^{3} \mathrm{~J}_{\mathrm{H}-\mathrm{H}}=6.8\right.$, $\left.{ }^{4} J_{\mathrm{H}-\mathrm{H}}=2.0 \mathrm{~Hz}, 2 \mathrm{H}, H 3\right), 4.46\left(\mathrm{t},{ }^{3} \int_{\mathrm{H}-\mathrm{H}}=2.0 \mathrm{~Hz}, 2 \mathrm{H}, \mathrm{H10}\right), 4.44\left(\mathrm{t},{ }^{3} \mathrm{~J}_{\mathrm{H}-\mathrm{H}}=2.0 \mathrm{~Hz}, 2 \mathrm{H}, H 11\right), 4.25\left(\mathrm{t},{ }^{3} \mathrm{~J}_{\mathrm{H}-\mathrm{H}}=2.0\right.$ $\mathrm{Hz}, 2 \mathrm{H}, \mathrm{H} 12), 4.22\left(\mathrm{t},{ }^{3} \mathrm{~J}_{\mathrm{H}-\mathrm{H}}=2.0 \mathrm{~Hz}, 2 \mathrm{H}, H 9\right), 2.49(\mathrm{~s}, 3 \mathrm{H}, \mathrm{H1}) \mathrm{ppm} ;{ }^{13} \mathrm{C}\left\{{ }^{1} \mathrm{H}\right\} \mathrm{NMR}\left(\mathrm{CDCl}_{3}, 298 \mathrm{~K}, 100 \mathrm{MHz}\right)$ : $\delta_{c}=138.7(C 5), 131.8$ (C4), 126.0 (C3), 120.2 (C2), 87 (C7), 86 (C6), 76.4 (C11), 74.0 (C12), 72.0 (C10), 70.9 (C9), 67.7 (C8), 41.3 (C13), 15.5 (C1) ppm; IR: 2206 (-C=C-) cm ${ }^{-1}$; MS ES+: calcd. for $\mathrm{C}_{19} \mathrm{H}_{15} \mathrm{FeSI}[\mathrm{M}]+$ 457.9289; found. 457.9296; EA calcd. C 49.81, H 3.30; found C 50.72, H 3.54.

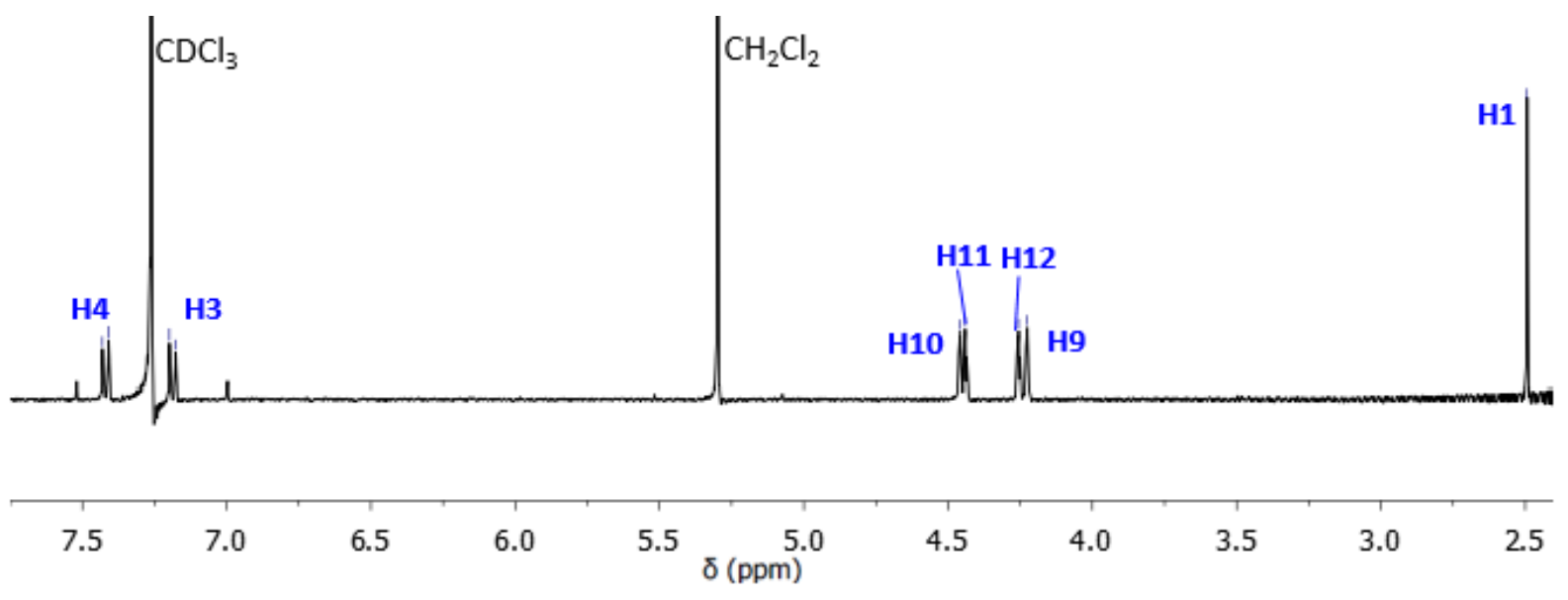

Figure S3: The ${ }^{1} \mathrm{H}$ NMR spectrum of $2 \mathrm{c}$ in $\mathrm{CDCl}_{3}$

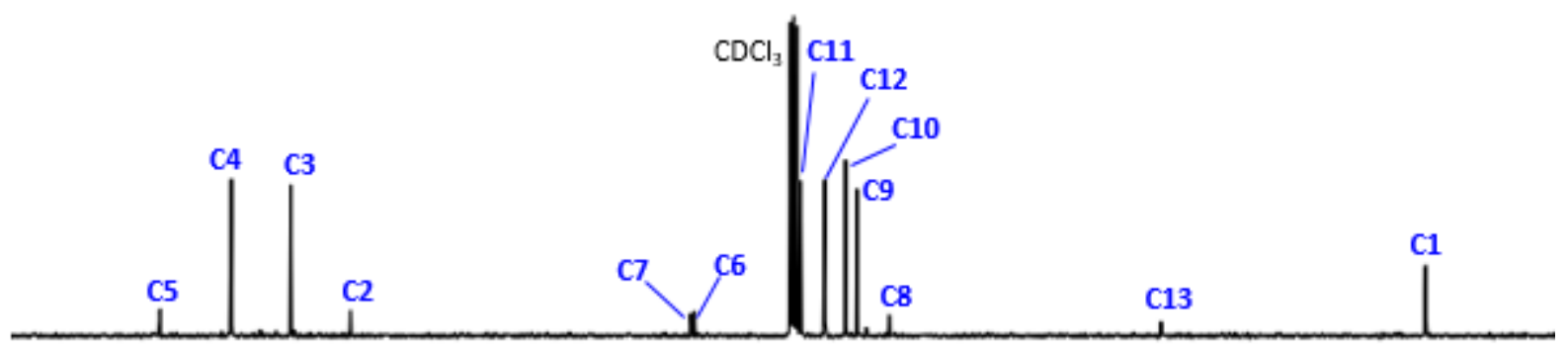

\begin{tabular}{|c|c|c|c|c|c|c|c|c|c|c|c|c|c|c|}
\hline 150 & 140 & 130 & 120 & 110 & 100 & 90 & 80 & 70 & 60 & 50 & 40 & 30 & 20 & 10 \\
\hline
\end{tabular}

Figure S4: The ${ }^{13} \mathrm{C}\left\{{ }^{1} \mathrm{H}\right\}$ NMR spectrum of $\mathbf{2} \mathbf{c}$ in $\mathrm{CDCl}_{3}$ 
1-lodo-1'-(3-(ethynyl)thioanisole)ferrocene: (2d) ${ }^{2}$

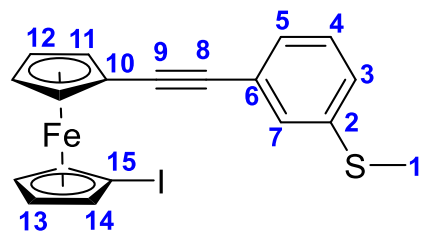

Following the general procedure, 1,1'-diiodoferrocene $(7.09 \mathrm{~g}, 16.20 \mathrm{mmol})$, Cul $(0.04 \mathrm{~g}, 0.20 \mathrm{mmol})$, 3-ethynylthioanisole $(0.60 \mathrm{~g}, 4.05 \mathrm{mmol})$ and $\mathrm{Pd}\left(\mathrm{P}^{\mathrm{t}} \mathrm{Bu}_{3}\right)_{2}(0.10 \mathrm{~g}, 0.20 \mathrm{mmol})$ gave a brown oil which was purified by chromatography on a silica column, eluted with $n$-hexane/DCM (1:0 $\rightarrow$ 4:1 v/v) to give the product as an orange solid (1.29 $\mathrm{g}, 11.34 \mathrm{mmol}, 70 \%)$.

${ }^{1} \mathrm{H}$ NMR $\left(\mathrm{CD}_{2} \mathrm{Cl}_{2}, 298 \mathrm{~K}, 400 \mathrm{MHz}\right): \delta_{\mathrm{H}}=7.41(\mathrm{~s}, 1 \mathrm{H}, \mathrm{H7}), 7.33-7.19(\mathrm{~m}, 3 \mathrm{H}, \mathrm{H3} / \mathrm{H} 4 / H 5), 4.51-4.45(\mathrm{~m}$, $4 \mathrm{H}, H 12 / H 13), 4.30-4.24(\mathrm{~m}, 4 \mathrm{H}, H 11 / H 14), 2.53(\mathrm{~s}, 3 \mathrm{H}, H 1) \mathrm{ppm} ;{ }^{13} \mathrm{C}\left\{{ }^{1} \mathrm{H}\right\} \mathrm{NMR}\left(\mathrm{CDCl}_{3}, 298 \mathrm{~K}, 100 \mathrm{MHz}\right)$ : $\delta_{C}=138.8(C 2), 129.0(C 3 / C 4 / C 5), 128.6$ (C3/C4/C5), 128.0 (C3/C4/C5), 126.0 (C7), 124.5 (C6), 87.5 (C9), 86.5 (C8), 76.4 (C13), 74.1 (C14), 72.1 (C12), 70.9 (C11), 67.4 (C10), 41.2 (C15), 15.8 (C1) ppm; IR:

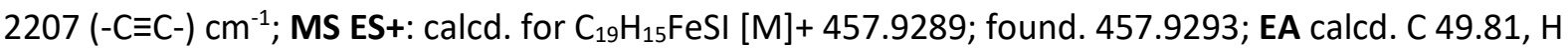
3.30; found C 50.41, H 3.19 .

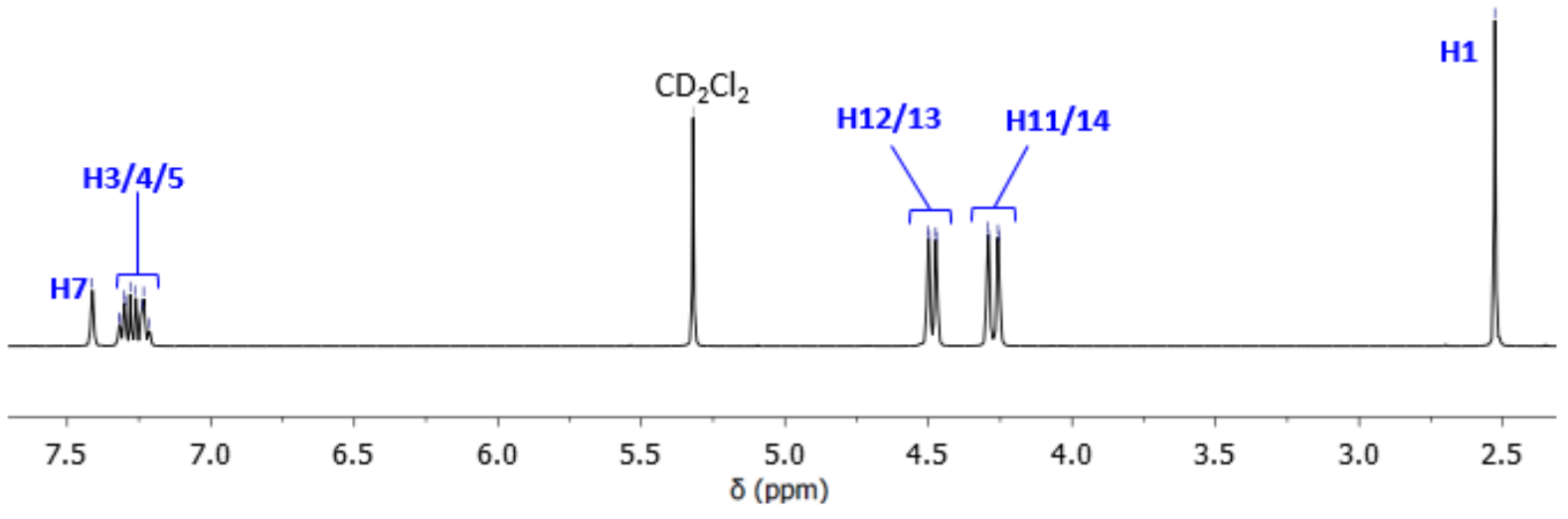

Figure S5: The ${ }^{1} \mathrm{H}$ NMR spectrum of $\mathbf{2 d}$ in $\mathrm{CD}_{2} \mathrm{Cl}_{2}$

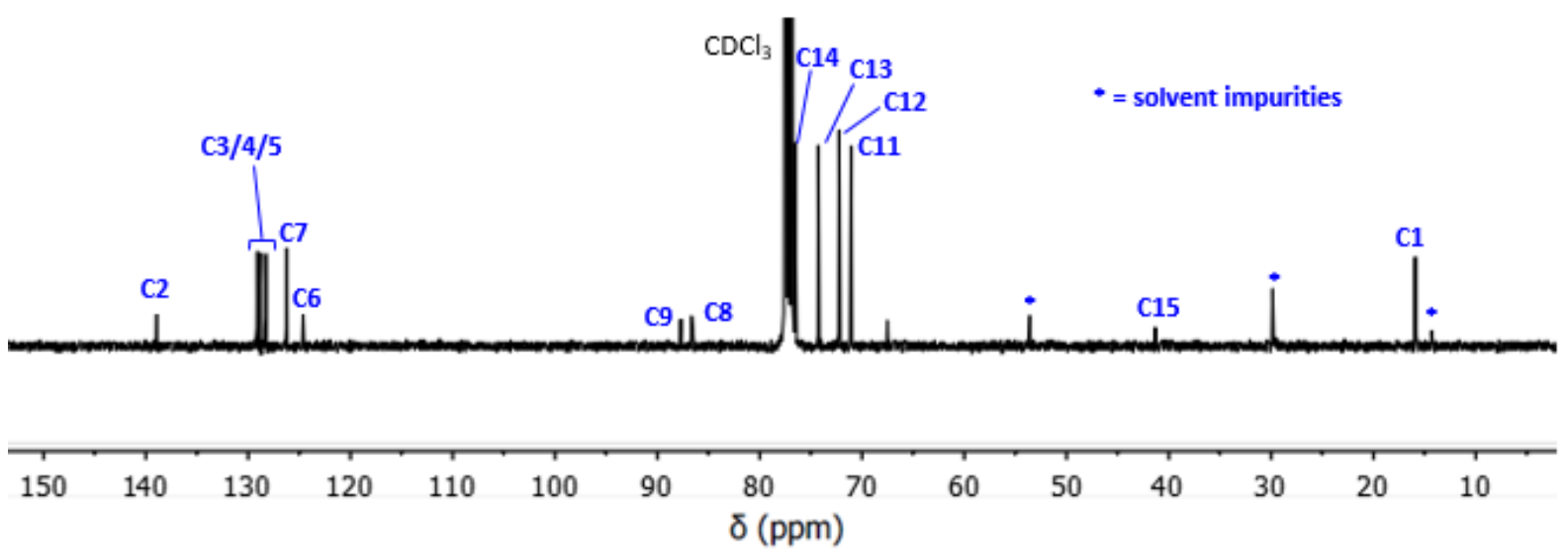

Figure S6: The ${ }^{13} \mathrm{C}\left\{{ }^{1} \mathrm{H}\right\}$ NMR spectrum of $\mathbf{2} \mathbf{d}$ in $\mathrm{CDCl}_{3}$ 
1,1'-Di(4-(ethynyl)pyridine)ferrocene: (3aa) ${ }^{3}$

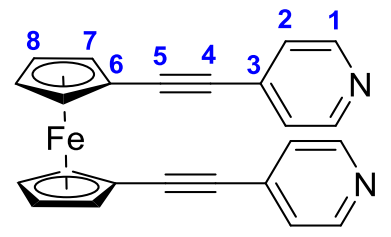

Following the general procedure, 1,1'-diiodoferrocene $(0.25 \mathrm{~g}, 0.57 \mathrm{mmol})$, Cul $(0.006 \mathrm{~g}, 0.029 \mathrm{mmol})$, $\mathrm{Pd}\left(\mathrm{P}^{\mathrm{t}} \mathrm{Bu}_{3}\right)_{2}(0.015 \mathrm{~g}, 0.029 \mathrm{mmol})$ and 4-ethynylpyridine hydrochloride $(0.175 \mathrm{~g}, 1.25 \mathrm{mmol})$ gave a black solid which was purified by chromatography on an alumina (V) column, eluted with $\mathrm{n}$ hexane/DCM (1:0 $\rightarrow 0: 1 \mathrm{v} / \mathrm{v})$ to give the product as an orange solid $(0.09 \mathrm{~g}, 0.22 \mathrm{mmol}, 70 \%)$.

${ }^{1} \mathrm{H}$ NMR $\left(\mathrm{CDCl}_{3}, 298 \mathrm{~K}, 400 \mathrm{MHz}\right): \delta_{\mathrm{H}} 8.49\left(\mathrm{dd},{ }^{3} \mathrm{~J}_{\mathrm{H}-\mathrm{H}}=4.4,{ }^{4} J_{\mathrm{H}-\mathrm{H}}=2 \mathrm{~Hz}, 4 \mathrm{H}, \mathrm{H} 2\right), 7.21\left(\mathrm{dd},{ }^{3} \mathrm{~J}_{\mathrm{H}-\mathrm{H}}=4.4,{ }^{4} \mathrm{~J}_{\mathrm{H}-\mathrm{H}}\right.$ $=2 \mathrm{~Hz}, 4 \mathrm{H}, H 1), 4.59\left(\mathrm{t},{ }^{3} \mathrm{~J}_{\mathrm{H}-\mathrm{H}}=2.0 \mathrm{~Hz}, 4 \mathrm{H}, H 8\right), 4.39\left(\mathrm{t},{ }^{3} \mathrm{H}_{\mathrm{H}-\mathrm{H}}=2.0 \mathrm{~Hz}, 4 \mathrm{H}, H 7\right) \mathrm{ppm} ;{ }^{13} \mathrm{C}\left\{{ }^{1} \mathrm{H}\right\} \mathrm{NMR}\left(\mathrm{CDCl}_{3}\right.$, $298 \mathrm{~K}, 100 \mathrm{MHz}$ ): $\delta_{\mathrm{c}} 149.5$ (C2), 132.0 (C3), 125.3 (C1), 92.5 (C4), 84.5 (C5), 73.5 (C8), 71.5 (C7), 66.0 (C6) ppm; IR: 2207 (-C $\equiv C-) \mathrm{cm}^{-1}$; MS ES+: calcd. for $\mathrm{C}_{24} \mathrm{H}_{17} \mathrm{FeN}_{2}$ [M]+ 389.0741; found. 389.0745; EA calcd. C 74.25, H 4.15, N 7.22; found C 72.92, H 4.45, N 7.01.

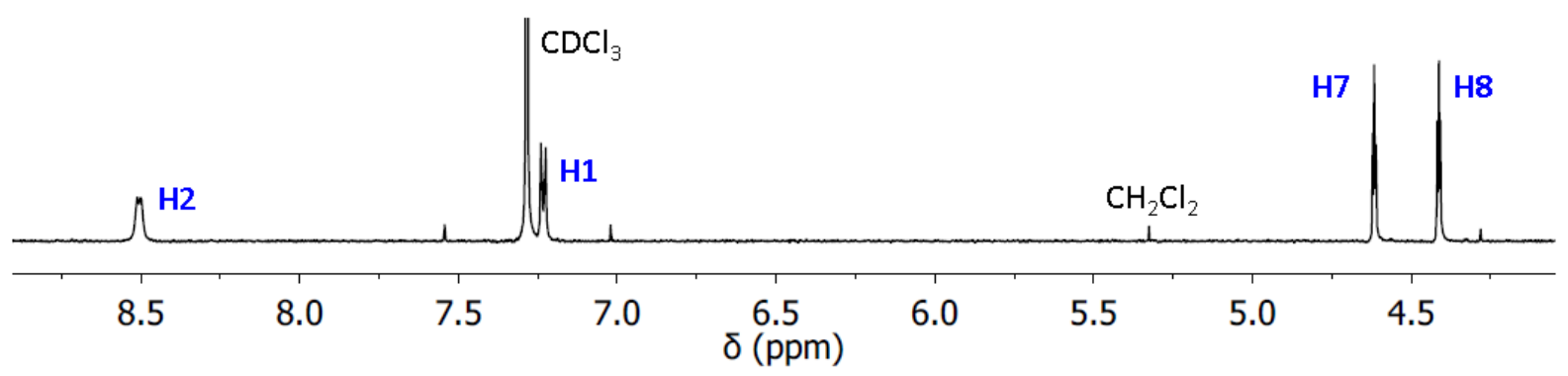

Figure S7: The ${ }^{1} \mathrm{H}$ NMR spectrum of 3 aa in $\mathrm{CDCl}_{3}$

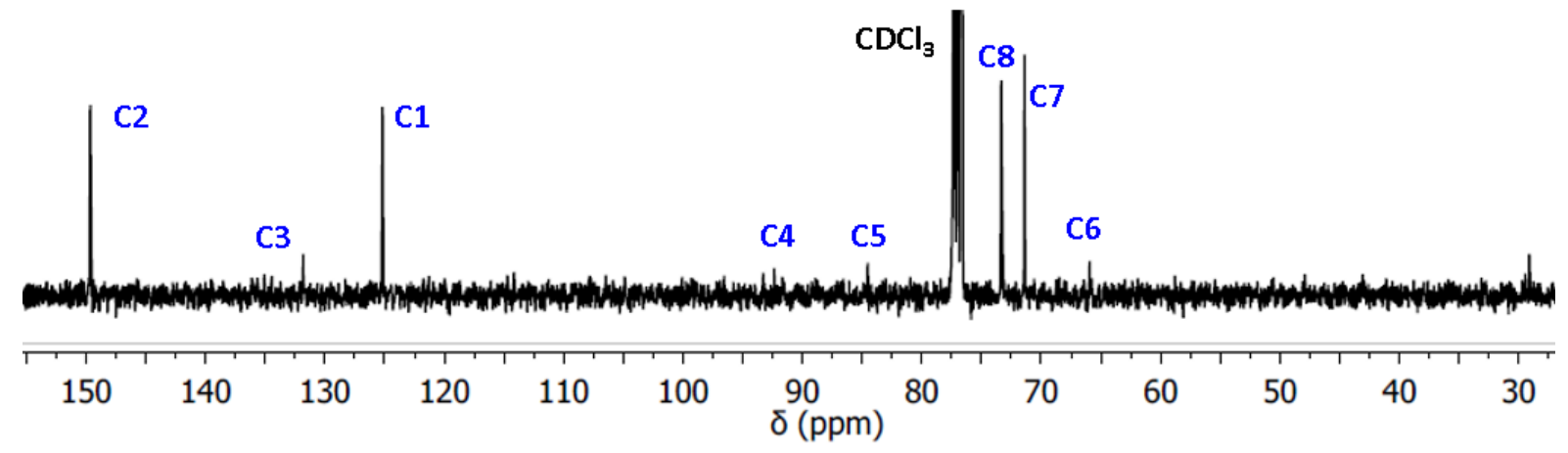

Figure S8: The ${ }^{13} \mathrm{C}\left\{{ }^{1} \mathrm{H}\right\}$ NMR spectrum of 3aa in $\mathrm{CDCl}_{3}$ 
1-(4-(Ethynyl)pyridine)-1'-(3-(ethynyl)pyridine)ferrocene: (3ab)

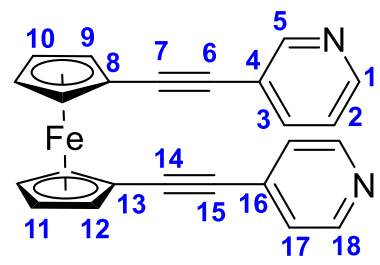

Following the general procedure, $(2 \mathrm{a})(0.30 \mathrm{~g}, 0.73 \mathrm{mmol})$, Cul $(0.01 \mathrm{~g}, 0.03 \mathrm{mmol}), \mathrm{Pd}\left(\mathrm{Pt}^{\mathrm{t}} \mathrm{Bu}_{3}\right)_{2}(0.02 \mathrm{~g}$, $0.04 \mathrm{mmol})$ and 3-ethynylpyridine $(0.15 \mathrm{~g}, 1.46 \mathrm{mmol})$ provided a black solid which was purified by chromatography on an alumina $(\mathrm{V})$ column, eluted with $\mathrm{n}$-hexane/DCM $(1: 0 \rightarrow 1: 1 \mathrm{v} / \mathrm{v})$ to give the product as an orange solid $(0.17 \mathrm{~g}, 0.45 \mathrm{mmol}, 61 \%)$.

${ }^{1} \mathrm{H}$ NMR $\left(\mathrm{CD}_{2} \mathrm{Cl}_{2}, 298 \mathrm{~K}, 400 \mathrm{MHz}\right): \delta_{\mathrm{H}} 8.57(\mathrm{~s}, 1 \mathrm{H}, \mathrm{H5}), 8.45-8.38(\mathrm{~m}, 3 \mathrm{H}, H 1, H 17), 7.58\left(\mathrm{dt},{ }^{3} \mathrm{~J}_{\mathrm{H}-\mathrm{H}}=8.4\right.$, $\left.{ }^{4} \mathrm{~J}_{\mathrm{H}-\mathrm{H}}=0.8 \mathrm{~Hz}, 1 \mathrm{H}, \mathrm{H3}\right), 7.17\left(\mathrm{dd},{ }^{3} \mathrm{~J}_{\mathrm{H}-\mathrm{H}}=4.8,{ }^{4} \mathrm{~J}_{\mathrm{H}-\mathrm{H}}=2.0 \mathrm{~Hz}, 2 \mathrm{H}, \mathrm{H1} 18\right), 7.12\left(\mathrm{ddd},{ }^{3} \mathrm{~J}_{\mathrm{H}-\mathrm{H}}=8.8,{ }^{3} \mathrm{~J}_{\mathrm{H}-\mathrm{H}}=7.6,{ }^{5} \mathrm{~J}_{\mathrm{H}-}\right.$ $\mathrm{H}=0.4 \mathrm{~Hz}, 1 \mathrm{H} . \mathrm{H2}$ ), 4.59 (pseudo-t, ${ }^{3} \mathrm{~J}_{\mathrm{H}-\mathrm{H}}=2.0 \mathrm{~Hz}, 2 \mathrm{H}, \mathrm{H10}$ ), $4.57\left(\mathrm{t},{ }^{3} \mathrm{~J}_{\mathrm{H}-\mathrm{H}}=2.0 \mathrm{~Hz}, 2 \mathrm{H}, H 11\right.$ ), 4.39 (pseudo$\left.\mathrm{t},{ }^{3} \mathrm{~J}_{\mathrm{H}-\mathrm{H}}=2.0 \mathrm{~Hz}, 2 \mathrm{H}, \mathrm{H9}\right), 4.36\left(\mathrm{t},{ }^{3} \mathrm{~J}_{\mathrm{H}-\mathrm{H}}=2.0 \mathrm{~Hz}, 2 \mathrm{H}, \mathrm{H12}\right) \mathrm{ppm} ;{ }^{13} \mathrm{C}\left\{{ }^{1} \mathrm{H}\right\} \mathrm{NMR}\left(\mathrm{CD}_{2} \mathrm{Cl}_{2}, 298 \mathrm{~K}, 100 \mathrm{MHz}\right)$ :

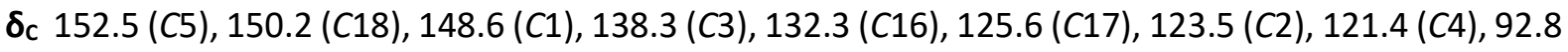
(C15), 90.6 (C7), 85.0 (C14), 84.2 (C6), 73.9 (C10), 73.7 (C11), 71.9 (C9), 71.6 (C12), 67.5 (C13), 66.6 (C8) ppm; IR: 2208 (-C $\equiv C-) \mathrm{cm}^{-1}$; MS ES+: calcd. for $\mathrm{C}_{24} \mathrm{H}_{17} \mathrm{FeN}_{2}$ [M]+ 389.0741; found. 389.0750; EA calcd. C 74.25, H 4.15, N 7.22; found C 73.94, H 4.16, N 6.89.

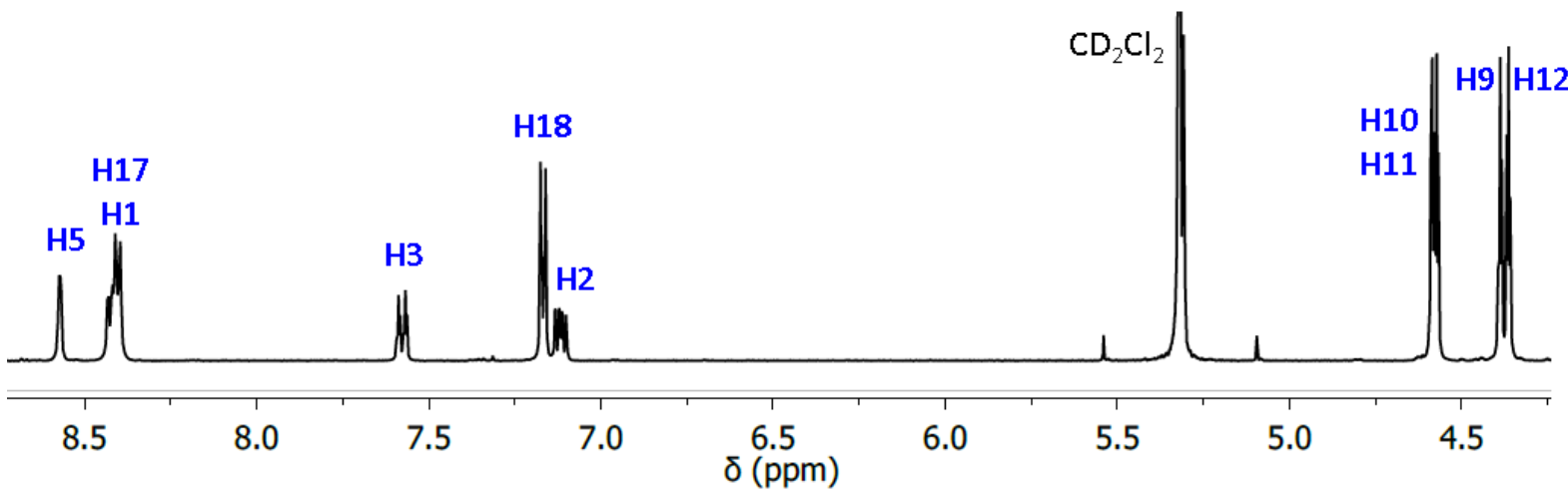

Figure S9: The ${ }^{1} \mathrm{H}$ NMR spectrum of $3 a b$ in $\mathrm{CD}_{2} \mathrm{Cl}_{2}$

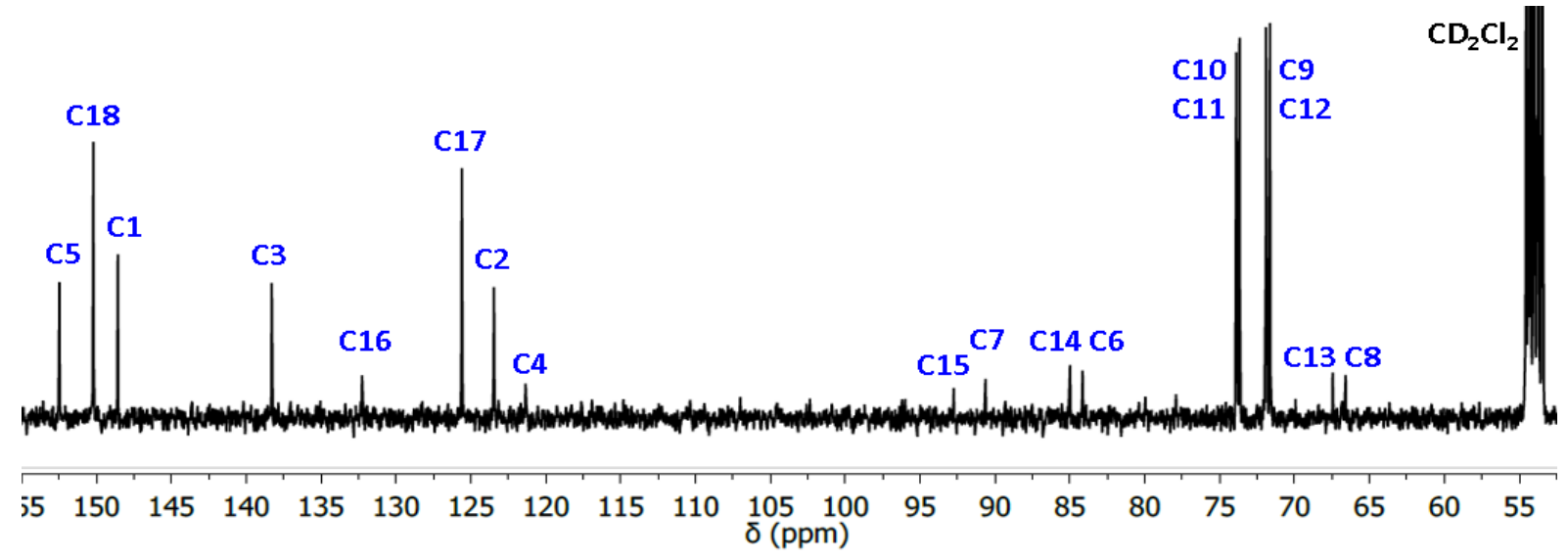

Figure S10: The ${ }^{13} \mathrm{C}\left\{{ }^{1} \mathrm{H}\right\}$ NMR spectrum of $3 a b$ in $\mathrm{CD}_{2} \mathrm{Cl}_{2}$ 
1-(4-(Ethynyl)thioanisole)-1'-(4-(ethynyl)pyridine)ferrocene: (3ac)

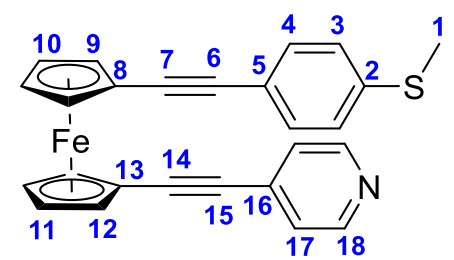

Following the general procedure, (2c) $(0.25 \mathrm{~g}, 0.49 \mathrm{mmol}), \mathrm{Cul}(0.02 \mathrm{mg}, 0.04 \mathrm{mmol})$, 4-ethynylpyridine $(0.07 \mathrm{mg}, 0.68 \mathrm{mmol})$ and $\mathrm{Pd}\left(\mathrm{P}^{\mathrm{t}} \mathrm{Bu}\right)_{2}(0.02 \mathrm{~g}, 0.04 \mathrm{mmol})$ yielded a brown solid which was purified by chromatography on a silica column, eluted with $n$-hexane/DCM $(1: 0 \rightarrow 0: 1 \mathrm{v} / \mathrm{v})$. The product was then washed with $\mathrm{n}$-hexane $(3 \times 100 \mathrm{~mL}$ ) before being dissolved in DCM, filtered through celite and then filtered through an alumina (V) plug which gave the product as an orange solid $(0.05 \mathrm{~g}, 0.17 \mathrm{mmol}$, $35 \%)$.

${ }^{1} \mathrm{H}$ NMR $\left(\mathrm{CDCl}_{3}, 298 \mathrm{~K}, 400 \mathrm{MHz}\right): \delta_{\mathrm{H}}=8.48(\mathrm{br} \mathrm{s}, 2 \mathrm{H}, H 18), 7.24\left(\mathrm{~d},{ }^{3} \mathrm{H}_{\mathrm{H}-\mathrm{H}}=8.4 \mathrm{~Hz}, 2 \mathrm{H}, H 4\right), 7.18(\mathrm{br}-\mathrm{d}$, ${ }^{3} J_{\mathrm{H}-\mathrm{H}}=4.0 \mathrm{~Hz}, 2 \mathrm{H}, \mathrm{H17}$ ), $7.09\left(\mathrm{~d},{ }^{3} \mathrm{~J}_{\mathrm{H}-\mathrm{H}}=8.4 \mathrm{~Hz}, 2 \mathrm{H}, H 3\right), 4.58$ (pseudo-t, ${ }^{3} \int_{\mathrm{H}-\mathrm{H}}=2.0 \mathrm{~Hz}, 2 \mathrm{H}, \mathrm{H} 11$ ), 4.55 (pseudo-t, ${ }^{3} \mathrm{~J}_{\mathrm{H}-\mathrm{H}}=2.0 \mathrm{~Hz}, 2 \mathrm{H}, \mathrm{H10}$ ), 4.37 (pseudo-t, ${ }^{3} \mathrm{H}_{\mathrm{H}-\mathrm{H}}=2.0 \mathrm{~Hz}, 2 \mathrm{H}, \mathrm{H} 12$ ), 4.32 (pseudo-t, ${ }^{3} \int_{\mathrm{H}-\mathrm{H}}=2.0$ $\mathrm{Hz}, 2 \mathrm{H}, H 9), 2.50(\mathrm{~s}, 3 \mathrm{H}, H 1) \mathrm{ppm} .{ }^{13} \mathrm{C}\left\{{ }^{1} \mathrm{H}\right\} \mathrm{NMR}\left(\mathrm{CDCl}_{3}, 298 \mathrm{~K}, 100 \mathrm{MHz}\right): \delta_{\mathrm{c}}=149.6(C 17), 138.9(\mathrm{C})$, 132.1 (C16), 131.7 (C4), $126.0(C 3), 125.5$ (C18), 120.1 (C2), 92.9 (C15), 86.9 and 86.8 (C6 and C7), 84.6 (C14), 73.4 (C11), 73.0 (C10), 71.4 (C12), 70.9 (C9), 67.9 (C8), 65.9 (C13), 15.6 (C1) ppm; IR: 2216 (-C=C) $\mathrm{cm}^{-1}$; MS ES+: calcd. for $\mathrm{C}_{26} \mathrm{H}_{20} \mathrm{FeNS}$ [M]+ 434.0666; found. 434.0669; EA calcd. C 72.06, H 4.42, N 3.23; found C 73.01, H 4.43, N 3.99.

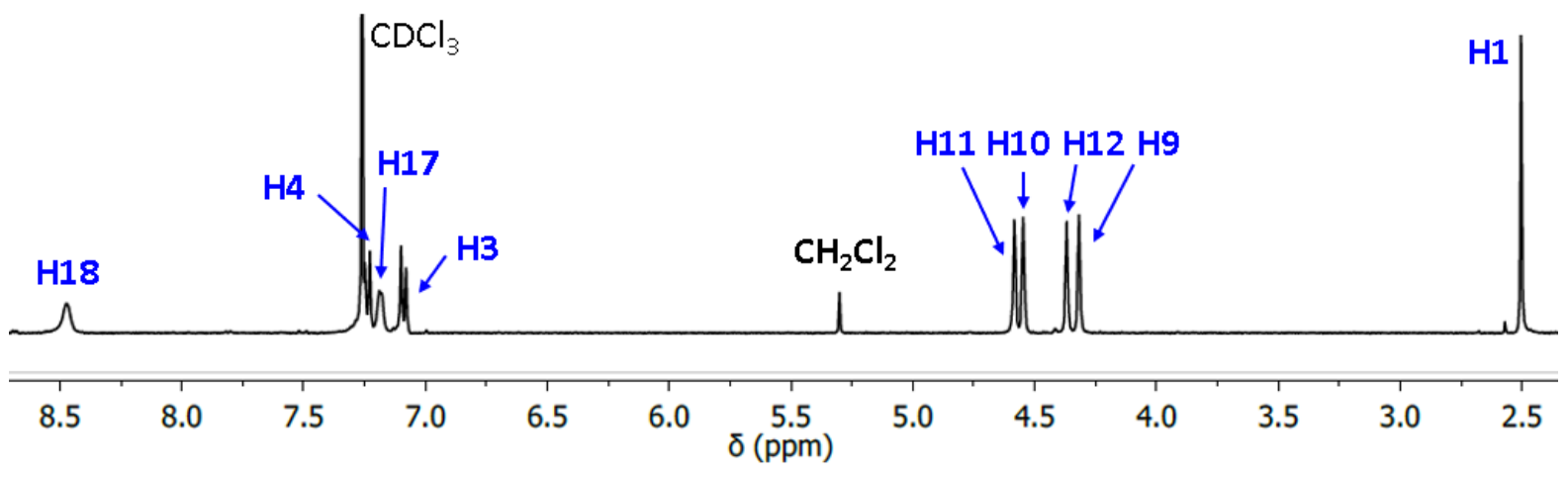

Figure S11: The ${ }^{1} \mathrm{H}$ NMR spectrum of $3 a \mathrm{ac}$ in $\mathrm{CDCl}_{3}$

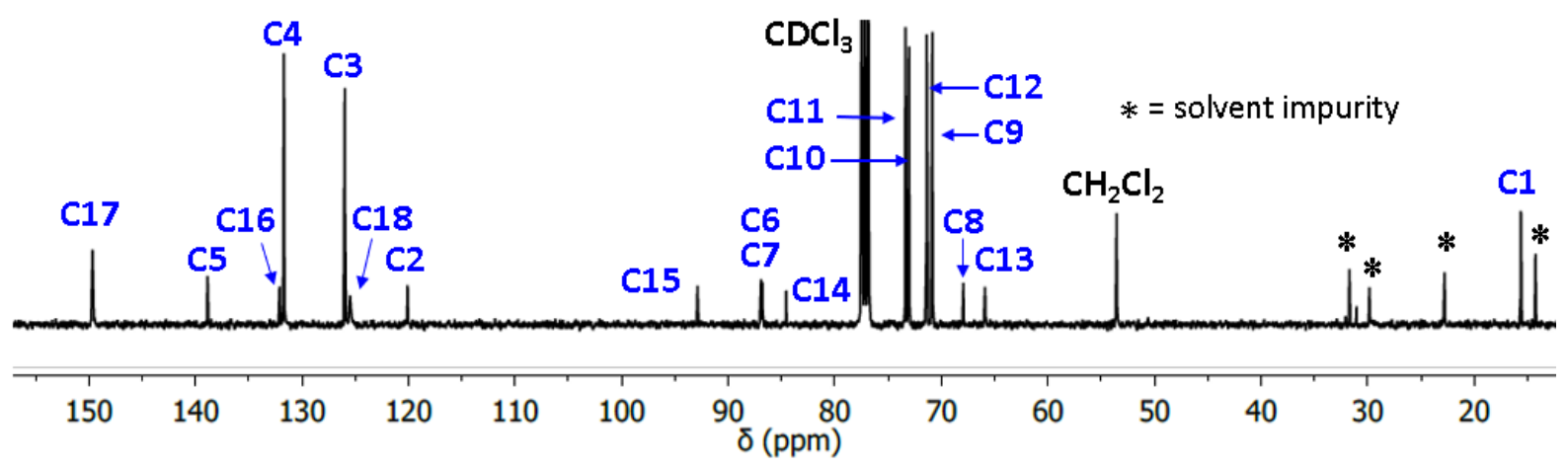

Figure S12: The ${ }^{13} \mathrm{C}\left\{{ }^{1} \mathrm{H}\right\}$ NMR spectrum of 3 ac in $\mathrm{CDCl}_{3}$ 
1-(3-(Ethynyl)thioanisole)-1'-(4-(ethynyl)pyridine)ferrocene: (3ad)

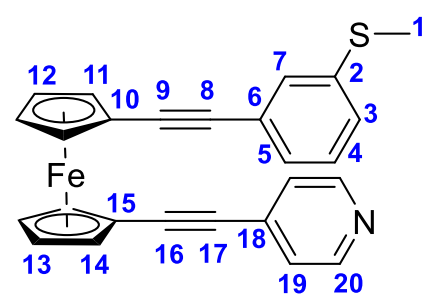

Following the general procedure, $(2 \mathrm{~d})(0.30 \mathrm{~g}, 0.73 \mathrm{mmol})$, Cul $(0.01 \mathrm{~g}, 0.03 \mathrm{mmol}), 3-$ ethynylthioanisole $(0.13 \mathrm{~g}, 0.87 \mathrm{mmol})$ and $\mathrm{Pd}\left(\mathrm{P}^{\mathrm{t}} \mathrm{Bu}_{3}\right)_{2}(0.02 \mathrm{~g}, 0.04 \mathrm{mmol})$ formed a black solid which was purified by chromatography on an alumina (V) column, eluted with n-hexane/DCM (1:0 $\rightarrow$ 4:6 $\mathrm{v} / \mathrm{v}$ ) to give the product as an orange solid $(0.15 \mathrm{~g}, 0.35 \mathrm{mmol}, 48 \%)$.

${ }^{1} \mathrm{H}$ NMR $\left(\mathrm{CDCl}_{3}, 298 \mathrm{~K}, 400 \mathrm{MHz}\right): \delta_{\mathrm{H}}=8.42(\mathrm{br} \mathrm{s}, 2 \mathrm{H}, \mathrm{H19}), 7.28(\mathrm{~m}, 1 \mathrm{H}, \mathrm{H} 5), 7.24(\mathrm{br}, 2 \mathrm{H}, \mathrm{H} 20) 7.16$ $\left(\mathrm{m}, 3 \mathrm{H}, H 3 / 4 / 7\right.$ ), 4.57 (pseudo-t, ${ }^{3}{ }_{\mathrm{H}-\mathrm{H}}=2.0 \mathrm{~Hz}, 2 \mathrm{H}, H 13$ ), 4.55 (pseudo-t, ${ }^{3} \mathrm{~J}_{\mathrm{H}-\mathrm{H}}=2.0 \mathrm{~Hz}, 2 \mathrm{H}, H 12$ ), 4.38 (pseudo-t, ${ }^{3} J_{\mathrm{H}-\mathrm{H}}=2.0 \mathrm{~Hz}, 2 \mathrm{H}, H 14$ ), 4.34 (pseudo-t, ${ }^{3} \mathrm{~J}_{\mathrm{H}-\mathrm{H}}=2.0 \mathrm{~Hz}, 2 \mathrm{H}, H 11$ ), $2.45(\mathrm{~s}, 3 \mathrm{H}, H 1) \mathrm{ppm} ;{ }^{13} \mathrm{C}\left\{{ }^{1} \mathrm{H}\right\}$ NMR $\left(\mathrm{CDCl}_{3}, 298 \mathrm{~K}, 100 \mathrm{MHz}\right): \delta_{\mathrm{c}}=149.6(C 19), 138.9(\mathrm{C2}), 132.0(\mathrm{C18}), 129.0(\mathrm{C} 5), 128.7 / 128.0(\mathrm{C} 4 / 6)$, 126.1 (C20), 126.0 (C3), 124.4 (C7), 92.7 (C17), 87.2 (C9), 86.8 (C8), 84.7 (C16), 73.4 (C12), 73.2 (C13),

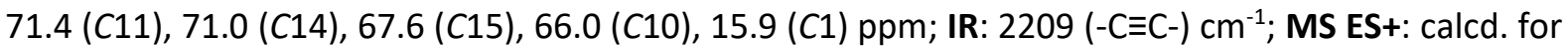
$\mathrm{C}_{26} \mathrm{H}_{10} \mathrm{FeNS}[\mathrm{M}]+434.0666$; found. 434.0681; EA calcd. C 72.06, H 4.42, N 3.23; found C 71.97, H 5.15, N 3.14 .

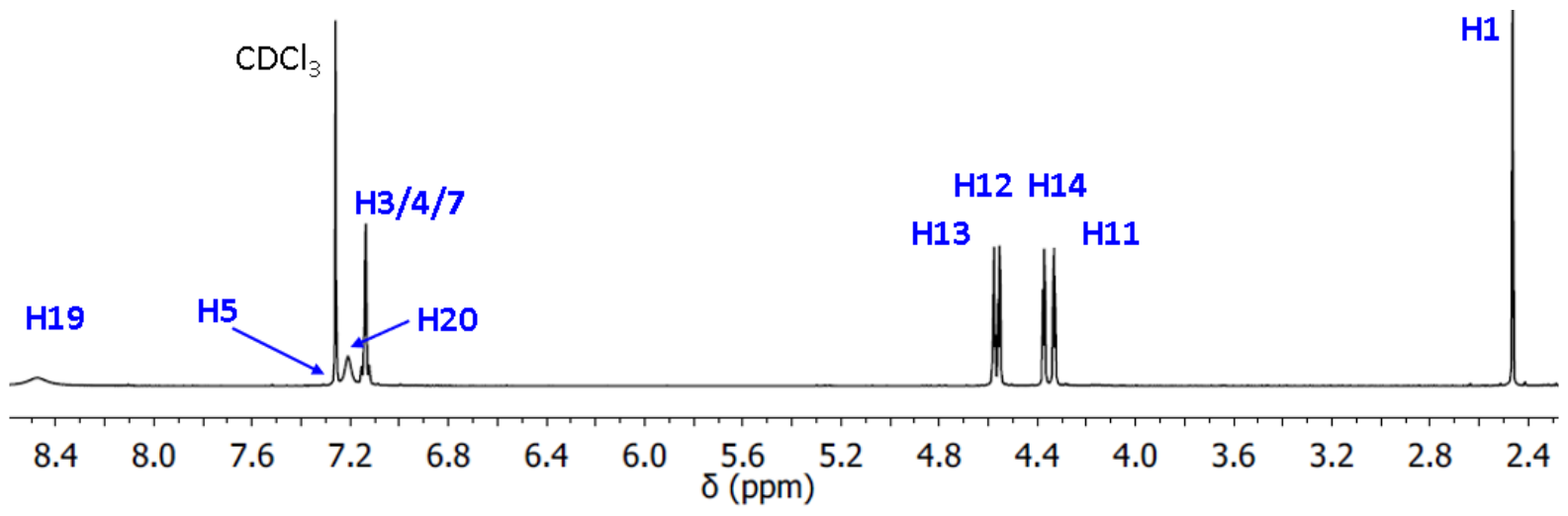

Figure S13: ${ }^{1} \mathrm{H}$ NMR spectrum of 3 ad in $\mathrm{CDCl}_{3}$

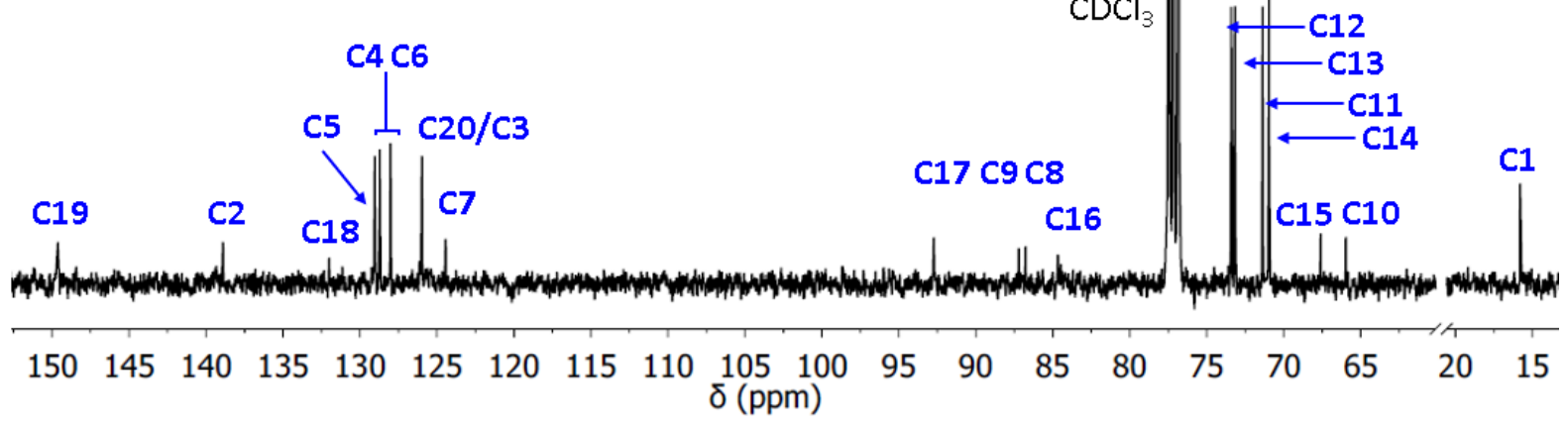

Figure $\mathrm{S} 14:{ }^{13} \mathrm{C}\left\{{ }^{1} \mathrm{H}\right\}$ NMR spectrum of 3 ad in $\mathrm{CDCl}_{3}$ 
1,1'-Di(3-(ethynyl)pyridine)ferrocene: (3bb) ${ }^{4}$

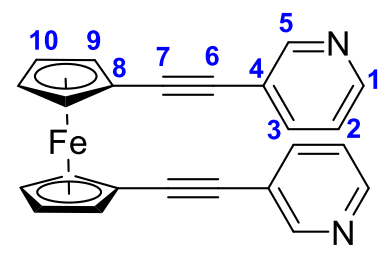

Following the general procedure, 1,1'-diiodoferrocene ( $1.15 \mathrm{~g}, 2.65 \mathrm{mmol})$, Cul $(0.04 \mathrm{~g}, 0.14 \mathrm{mmol}), 3$ ethynylpyridine $(0.60 \mathrm{~g}, 5.83 \mathrm{mmol})$ and $\mathrm{Pd}\left(\mathrm{P}^{\mathrm{t} B \mathrm{Bu}_{3}}\right)_{2}(0.07 \mathrm{~g}, 0.14 \mathrm{mmol})$ yielded a brown powder which was purified by chromatography on a silica column, eluted with $n$-hexane/DCM/Acetone (1:0:0 $\rightarrow$ $0: 1: 1 \mathrm{v} / \mathrm{v} / \mathrm{v})$. The fractions containing the product were then washed with distilled water $(3 \times 100 \mathrm{~mL})$ and brine $(100 \mathrm{~mL})$ to yield the product as an orange solid $(0.76 \mathrm{~g}, 1.31 \mathrm{mmol}, 45 \%)$.

${ }^{1} \mathrm{H}$ NMR $\left(\mathrm{CDCl}_{3}, 298 \mathrm{~K}, 400 \mathrm{MHz}\right): \delta_{\mathrm{H}}=8.65(\mathrm{br} \mathrm{s}, 2 \mathrm{H}, \mathrm{H5}), 8.49\left(\mathrm{~d},{ }^{3} \mathrm{~J}_{\mathrm{H}-\mathrm{H}}=4.0 \mathrm{~Hz}, 2 \mathrm{H}, H 1\right), 7.65\left(\mathrm{dt},{ }^{3} \mathrm{~J}_{\mathrm{H}-\mathrm{H}}\right.$ $\left.=8.4,{ }^{4} J_{\mathrm{H}-\mathrm{H}}=2.0 \mathrm{~Hz}, 2 \mathrm{H}, H 3\right), 7.17\left(\mathrm{dd},{ }^{3} \mathrm{~J}_{\mathrm{H}-\mathrm{H}}=8.0,{ }^{4} \mathrm{~J}_{\mathrm{H}-\mathrm{H}}=2.0 \mathrm{~Hz}, 2 \mathrm{H}, \mathrm{H2}\right.$ ), 4.60 (pseudo-t, ${ }^{3} \int_{\mathrm{H}-\mathrm{H}}=2.0 \mathrm{~Hz}$, $4 \mathrm{H}, \mathrm{H10}), 4.39$ (pseudo-t, $\left.{ }^{3} \mathrm{~J}_{\mathrm{H}-\mathrm{H}}=2.0 \mathrm{~Hz}, 4 \mathrm{H}, \mathrm{H9}\right) \mathrm{ppm} ;{ }^{13} \mathrm{C}\left\{{ }^{1} \mathrm{H}\right\} \mathrm{NMR}\left(\mathrm{CDCl}_{3}, 298 \mathrm{~K}, 100 \mathrm{MHz}\right): \delta_{\mathrm{C}}=152.0$ (C5), 147.9 (C1), 138.0 (C3), 123.0 (C2), 120.9 (C4), 90.5 (C7), 83.6 (C6), 73.1 (C10), 71.1 (C9), 66.6 (C8) ppm; IR: 2206 (-C三C-) cm cm $^{-1}$ MS ES+: calcd. for $\mathrm{C}_{24} \mathrm{H}_{17} \mathrm{FeN}_{2}$ [M]+ 389.0741; found. 389.0749; EA calcd. C 74.25, H 4.15, N 7.22; found C 74.09, H 4.25, N 7.06.

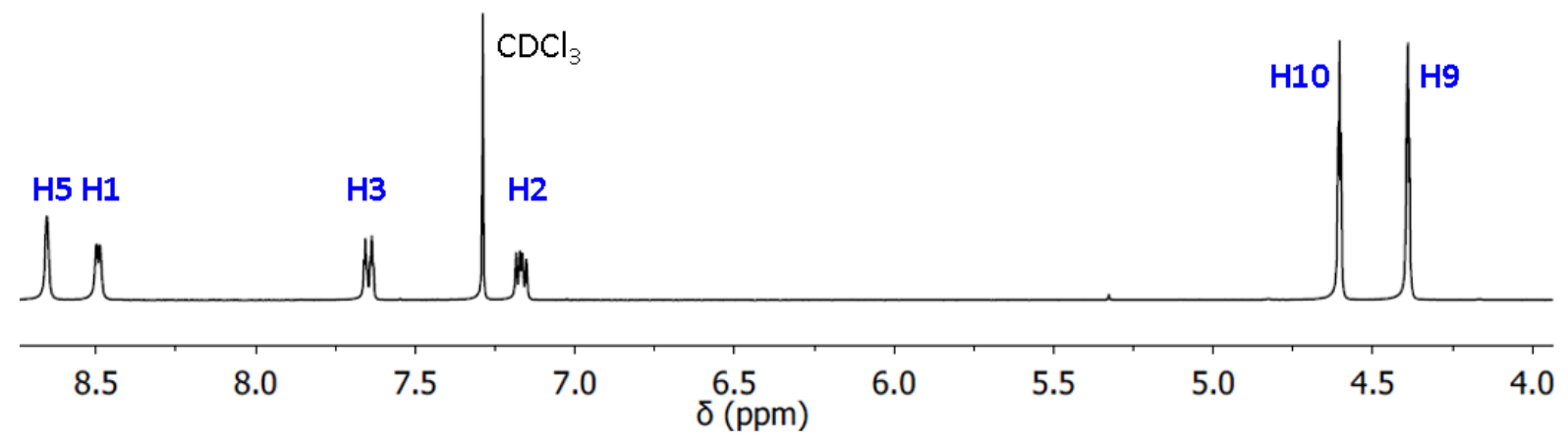

Figure S15: The ${ }^{1} \mathrm{H}$ NMR spectrum of $\mathbf{3 b b}$ in $\mathrm{CDCl}_{3}$

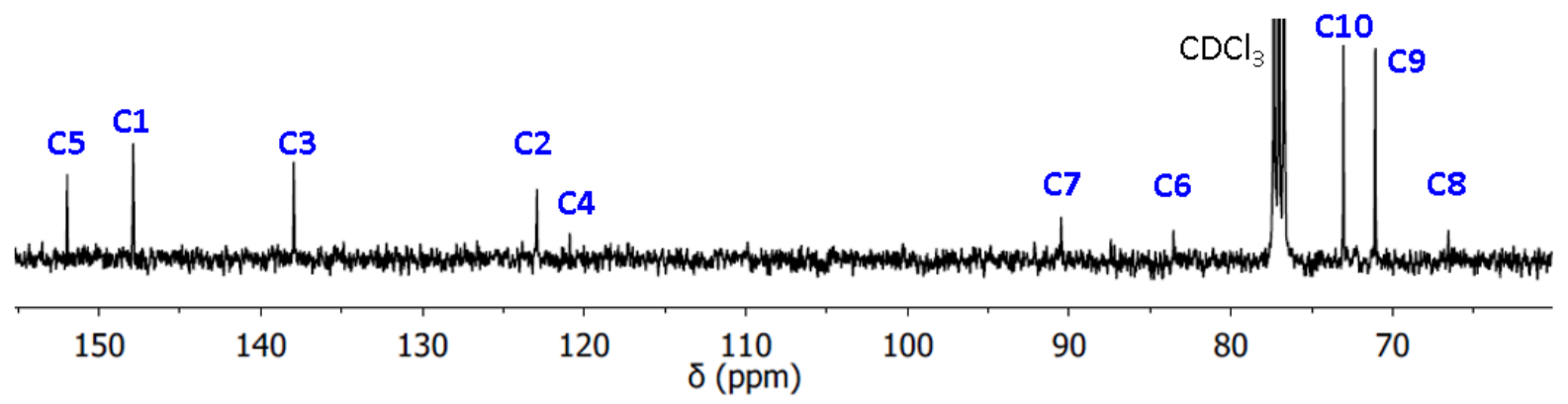

Figure S16: The ${ }^{13} \mathrm{C}\left\{{ }^{1} \mathrm{H}\right\}$ NMR spectrum of $\mathbf{3 b b}$ in $\mathrm{CDCl}_{3}$ 
1-(4-(Ethynyl)thioanisole)-1'-(3-(ethynyl)pyridine)ferrocene: (3bc)

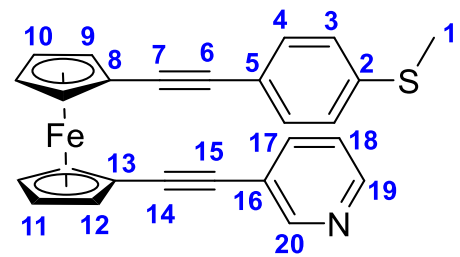

Following the general procedure, (2c) $(0.250 \mathrm{~g}, 0.550 \mathrm{mmol})$, Cul $(0.01 \mathrm{~g}, 0.03 \mathrm{mmol})$, 3ethynylpyridine $(0.07 \mathrm{~g}, 0.66 \mathrm{mmol})$ and $\mathrm{Pd}\left(\mathrm{P}^{\mathrm{t}} \mathrm{Bu}_{3}\right)_{2}(0.01 \mathrm{~g}, 0.03 \mathrm{mmol})$ gave a black solid which was purified by chromatography on an alumina $(\mathrm{V})$ column, eluted with $\mathrm{n}$-hexane/DCM $(1: 0 \rightarrow 1: 1 \mathrm{v} / \mathrm{v})$ to give the product as an orange solid $(0.17 \mathrm{~g}, 0.40 \mathrm{mmol}, 73 \%)$.

${ }^{1} \mathrm{H}$ NMR $\left(\mathrm{CDCl}_{3}, 298 \mathrm{~K}, 400 \mathrm{MHz}\right): \delta_{\mathrm{H}}=8.67(\mathrm{br}, 1 \mathrm{H}, \mathrm{H} 20), 8.48(\mathrm{br}, 1 \mathrm{H}, H 19), 7.60\left(\mathrm{dt},{ }^{3} \int_{\mathrm{H}-\mathrm{H}}=8.0,{ }^{4} J_{\mathrm{H}-\mathrm{H}}=\right.$ $0.8 \mathrm{~Hz}, 1 \mathrm{H}, H 17), 7.29\left(\mathrm{~d}, 3^{3} \mathrm{H}_{\mathrm{H}}=8.0,2 \mathrm{H}, H 4\right), 7.13(\mathrm{br}, 1 \mathrm{H}, \mathrm{H1} 18), 7.08\left(\mathrm{~d}, 3_{\mathrm{H}-\mathrm{H}}=8.0,2 \mathrm{H}, H 3\right), 4.56$ (pseudo-t, ${ }^{3} J_{\mathrm{H}-\mathrm{H}}=2.0 \mathrm{~Hz}, 2 \mathrm{H}, \mathrm{H11}$ ), 4.54 (pseudo-t, ${ }^{3} J_{\mathrm{H}-\mathrm{H}}=2.0 \mathrm{~Hz}, 2 \mathrm{H}, \mathrm{H} 10$ ), 4.35 (pseudo-t, ${ }^{3} \int_{\mathrm{H}-\mathrm{H}}=2.0$ $\mathrm{Hz}, 2 \mathrm{H}, \mathrm{H} 12), 4.32\left(\mathrm{t},{ }^{3} \mathrm{~J}_{\mathrm{H}-\mathrm{H}}=2.0 \mathrm{~Hz}, 2 \mathrm{H}, H 9\right), 2.48(\mathrm{~s}, 3 \mathrm{H}, \mathrm{H1}) \mathrm{ppm} ;{ }^{13} \mathrm{C}\left\{{ }^{1} \mathrm{H}\right\} \mathrm{NMR}\left(\mathrm{CDCl}_{3}, 298 \mathrm{~K}, 100 \mathrm{MHz}\right)$ : $\delta_{\mathrm{C}}=152.0(C 20), 147.7(C 19), 138.5(C 5), 138.0(C 17), 131.6$ (C4), $126.0(C 3), 123.2$ (C18), 122.2 (C16), 120.0 (C2), 90.8 (C14), 86.8 (C7), 86.7 (C6), 83.5 (C15), 73.0 (C10), 72.9 (C11), $71.0(C 12), 70.8(C 9)$, 67.6 (C8), 66.4 (C13), 15.5 (C1) ppm; IR: 2209 (-C=C-) cm ${ }^{-1}$; MS ES+: calcd. for $\mathrm{C}_{26} \mathrm{H}_{10} \mathrm{FeNS}$ [M]+ 434.0666; found. 434.0658; EA calcd. C 72.06, H 4.42, N 3.23; found C 71.45, H 4.46, N 3.62.

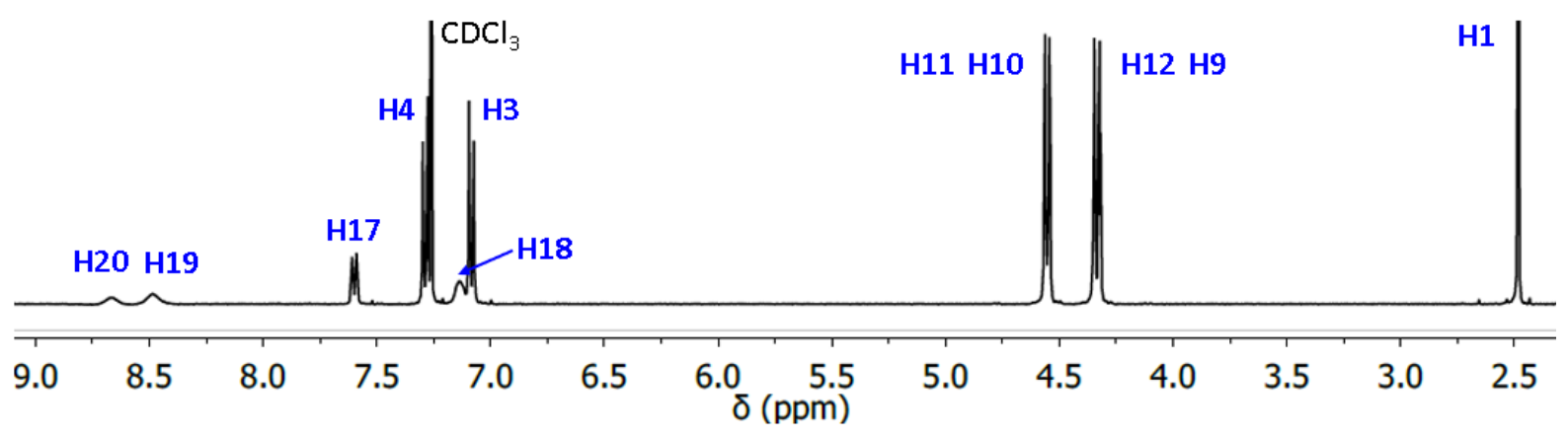

Figure S17: The ${ }^{1} \mathrm{H}$ NMR spectrum of $3 \mathbf{b c}$ in $\mathrm{CDCl}_{3}$

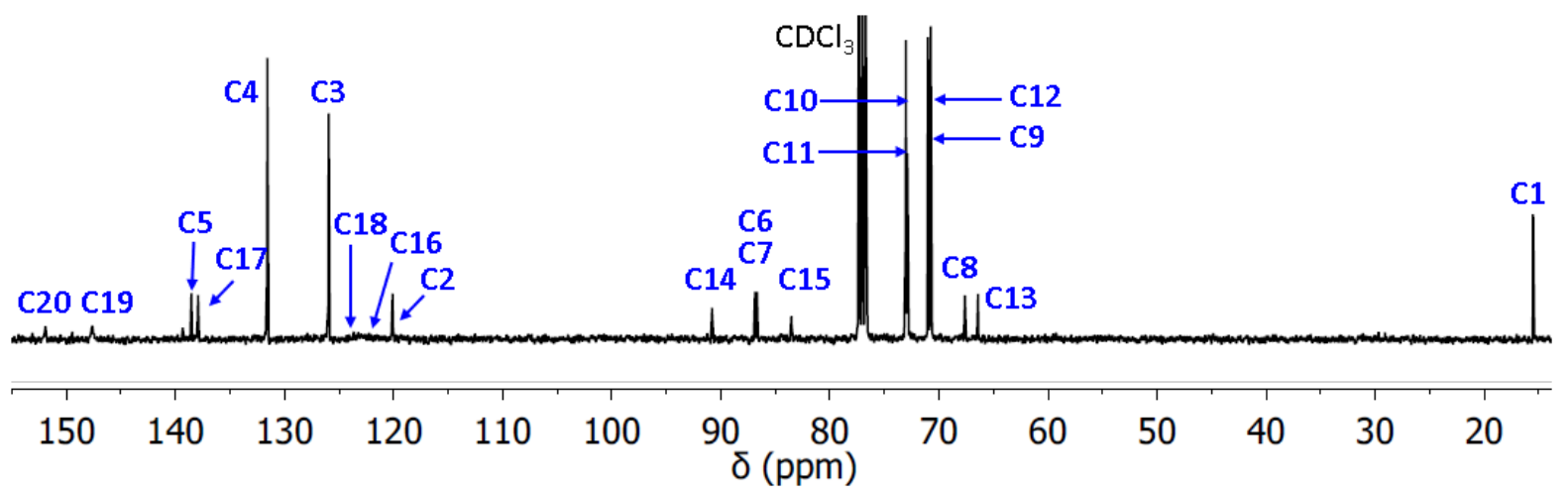

Figure S18: The ${ }^{13} \mathrm{C}\left\{{ }^{1} \mathrm{H}\right\}$ NMR spectrum of $\mathbf{3 b c}$ in $\mathrm{CDCl}_{3}$ 
1-(3-(Ethynyl)thioanisole)-1'-(3-(ethynyl)pyridine)ferrocene: (3bd)

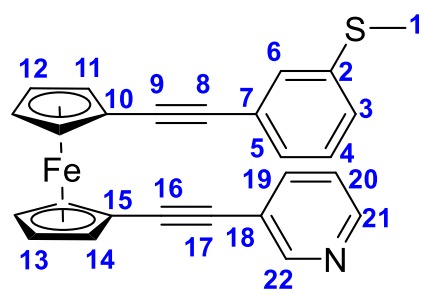

Following the aforementioned general procedure, (2d) $(0.97 \mathrm{~g}, 2.14 \mathrm{mmol})$, Cul $(0.05 \mathrm{~g}, 0.25 \mathrm{mmol})$, 3-ethynylpyridine $(0.09 \mathrm{~g}, 0.89 \mathrm{mmol})$ and $\mathrm{Pd}\left(\mathrm{P}^{\mathrm{t}} \mathrm{Bu}_{3}\right)_{2}(0.02 \mathrm{~g}, 0.25 \mathrm{mmol})$ gave a black oil which was purified by chromatography on a silica column, eluted with $n$-hexane/DCM $(1: 0 \rightarrow 1: 4 \mathrm{v} / \mathrm{v})$ to give the product as an orange solid $(0.29 \mathrm{~g}, 0.68 \mathrm{mmol}, 76 \%)$.

${ }^{1} \mathrm{H}_{\mathrm{NMR}}\left(\mathrm{CDCl}_{3}, 298 \mathrm{~K}, 400 \mathrm{MHz}\right): \delta_{\mathrm{H}}=8.60(\mathrm{br} \mathrm{s}, 1 \mathrm{H}, \mathrm{H19} / 20), 7.61\left(\mathrm{dt},{ }^{3} \mathrm{~J}_{\mathrm{H}-\mathrm{H}}=8.0,{ }^{4} \mathrm{~J}_{\mathrm{H}-\mathrm{H}}=0.8 \mathrm{~Hz}, 1 \mathrm{H}\right.$, $H 22), 7.27(\mathrm{~s}, 1 \mathrm{H}, H 6), 7.18-7.11(\mathrm{~m}, 4 \mathrm{H}, H 21 / 3 / 4 / 5), 4.58(\mathrm{~m}, 4 \mathrm{H}, H 12 / 13), 4.38$ (pseudo-t, ${ }^{3} \mathrm{~J}_{\mathrm{H}-\mathrm{H}}=2.0$ $\mathrm{Hz}, 2 \mathrm{H}, \mathrm{H11}$ ), 4.36 (pseudo-t, $\left.{ }^{3} \mathrm{H}_{\mathrm{H}-\mathrm{H}}=2.0 \mathrm{~Hz}, 2 \mathrm{H}, \mathrm{H} 14\right), 2.48(\mathrm{~s}, 3 \mathrm{H}, \mathrm{H1}) \mathrm{ppm} ;{ }^{13} \mathrm{C}\left\{{ }^{1} \mathrm{H}\right\} \mathrm{NMR}\left(\mathrm{CDCl}_{3}, 298 \mathrm{~K}\right.$, $100 \mathrm{MHz}$ ): $\delta_{\mathrm{C}}=152.0(C 22), 147.9$ (C21), $138.9(C 7), 138.0(C 18), 129.0,128.7,128.1(C 3 / 4 / 5), 126.1$ (C6), 124.5 (C2), 90.9 (C16), 87.5 (C9), 86.6 (C8), 83.7 (C17), 73.273 .1 (C12/13), 71.2, 71.0 (C11/14), 67.4 (C15), 66.5 (C10), 15.8 (C1) ppm; IR: 2209 (-C $\equiv C-) \mathrm{cm}^{-1}$; MS ES+: calcd. for $\mathrm{C}_{26} \mathrm{H}_{19} \mathrm{FeNS}$ [M]+ 434.0666; found. 434.0668; EA calcd. C 72.06, H 4.42, N 3.23; found C 71.85, H 4.49, N 3.30.

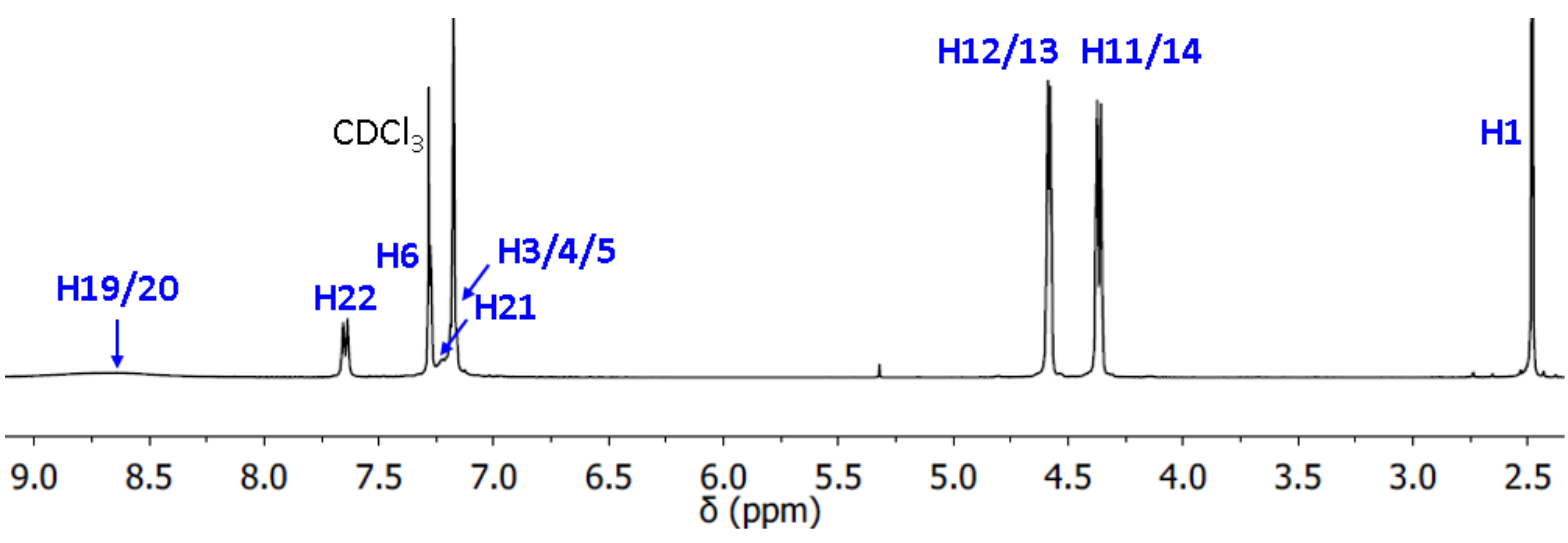

Figure S19: ${ }^{1} \mathrm{H}$ NMR spectrum of $3 \mathrm{bd}$ in $\mathrm{CDCl}_{3}$

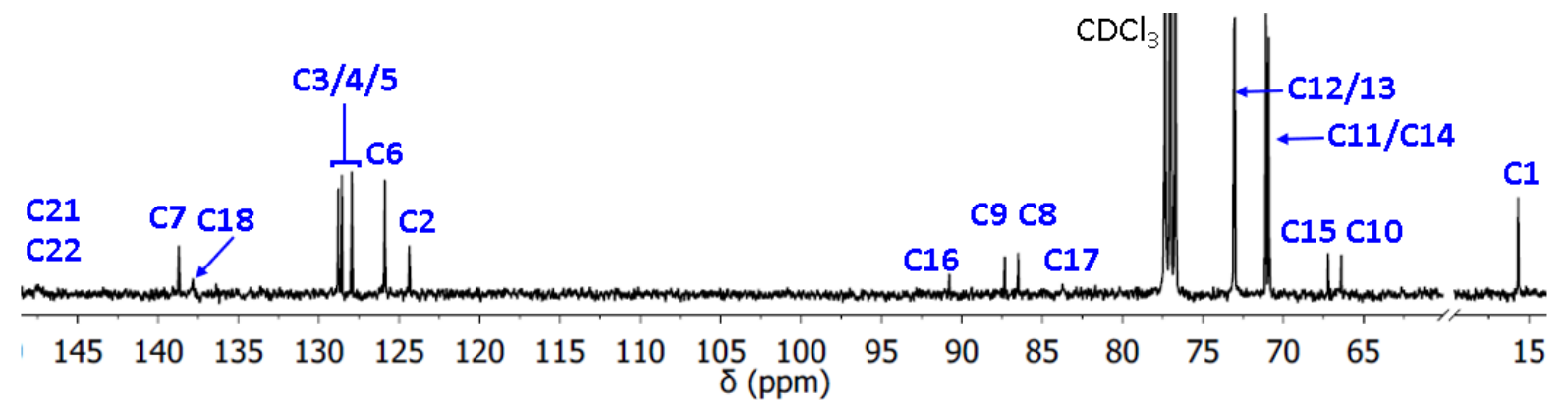

Figure S20: ${ }^{13} \mathrm{C}\left\{{ }^{1} \mathrm{H}\right\}$ NMR spectrum of $\mathbf{3 b d}$ in $\mathrm{CDCl}_{3}$ 
1,1'-Di(4-(ethynyl)thioanisole)ferrocene: (3cc) ${ }^{5}$

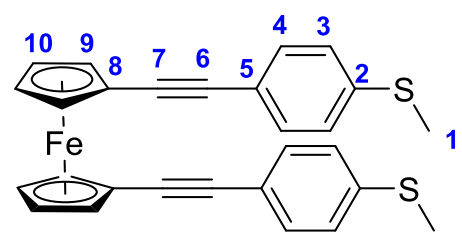

Following the general procedure, 1,1'-diiodoferrocene $(0.25 \mathrm{~g}, 0.57 \mathrm{mmol})$, Cul $(0.01 \mathrm{~g}, 0.057 \mathrm{mmol})$, 4-ethynylthioanisole $(0.30 \mathrm{~g}, 2.0 \mathrm{mmol})$ and $\mathrm{Pd}\left(\mathrm{P}^{\mathrm{t}} \mathrm{Bu}_{3}\right)_{2}(0.03 \mathrm{~g}, 0.057 \mathrm{mmol})$ provided a brown oil which was purified by chromatography on a silica column, eluted with $n$-hexane/DCM $(1: 0 \rightarrow 3: 2 \mathrm{v} / \mathrm{v})$ to give the product as an orange solid $(0.18 \mathrm{~g}, 0.37 \mathrm{mmol}, 65 \%)$.

${ }^{1} \mathrm{H}$ NMR $\left(\mathrm{CDCl}_{3}, 298 \mathrm{~K}, 400 \mathrm{MHz}\right): \delta_{\mathrm{H}}=7.32(\mathrm{~m}, 4 \mathrm{H}, \mathrm{H4}), 7.12(\mathrm{~m}, 4 \mathrm{H}, \mathrm{H3}), 4.56$ (pseudo-t, ${ }^{3} \mathrm{~J}_{\mathrm{H}-\mathrm{H}}=2.0 \mathrm{~Hz}$, $4 \mathrm{H}, \mathrm{H10}$ ), 4.33 (pseudo-t, $\left.{ }^{3} \mathrm{~J}_{\mathrm{H}-\mathrm{H}}=2.0 \mathrm{~Hz}, 4 \mathrm{H}, \mathrm{H9}\right), 2.50(\mathrm{~s}, 6 \mathrm{H}, \mathrm{H1}) \mathrm{ppm} ;{ }^{13} \mathrm{C}\left\{{ }^{1} \mathrm{H}\right\} \mathrm{NMR}\left(\mathrm{CDCl}_{3}, 298 \mathrm{~K}, 100\right.$ $\mathrm{MHz}$ : $\delta_{\mathrm{c}}=138.4(C 5), 131.7(C 4), 125.8(C 3), 120.1$ (C2), 87.1 (C7), 86.5 (C6), 72.8 (C10), 70.7 (C9), 67.4 (C8), 15.5 (C1) ppm; IR: 2208 (-CEC-) cm-1; MS ES+: calcd. for $\mathrm{C}_{28} \mathrm{H}_{22} \mathrm{FeS}_{2}[\mathrm{M}]+478.0512$; found. 478.0522. EA for $\mathrm{C}_{28} \mathrm{H}_{22} \mathrm{FeS}_{2} . \mathrm{H}_{2} \mathrm{O}$ calcd. C 70.29, $\mathrm{H}$ 4.63; found $\mathrm{C} 69.75, \mathrm{H} 4.73$.

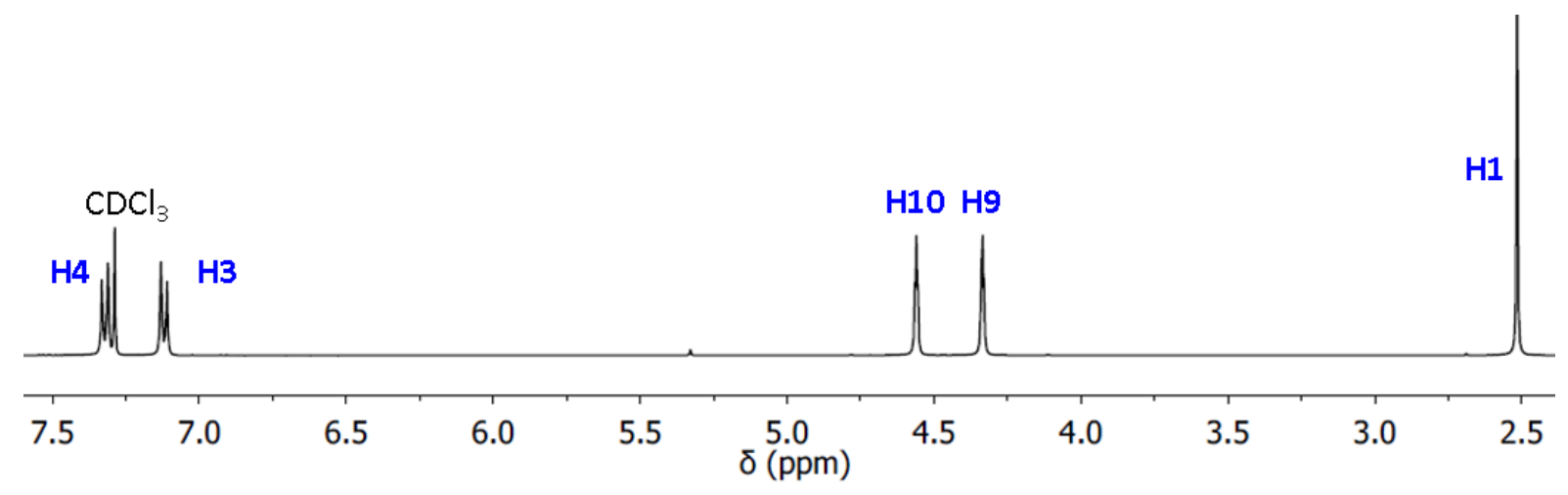

Figure S21: The ${ }^{1} \mathrm{H}$ NMR spectrum of $3 \mathrm{cc}$ in $\mathrm{CDCl}_{3}$

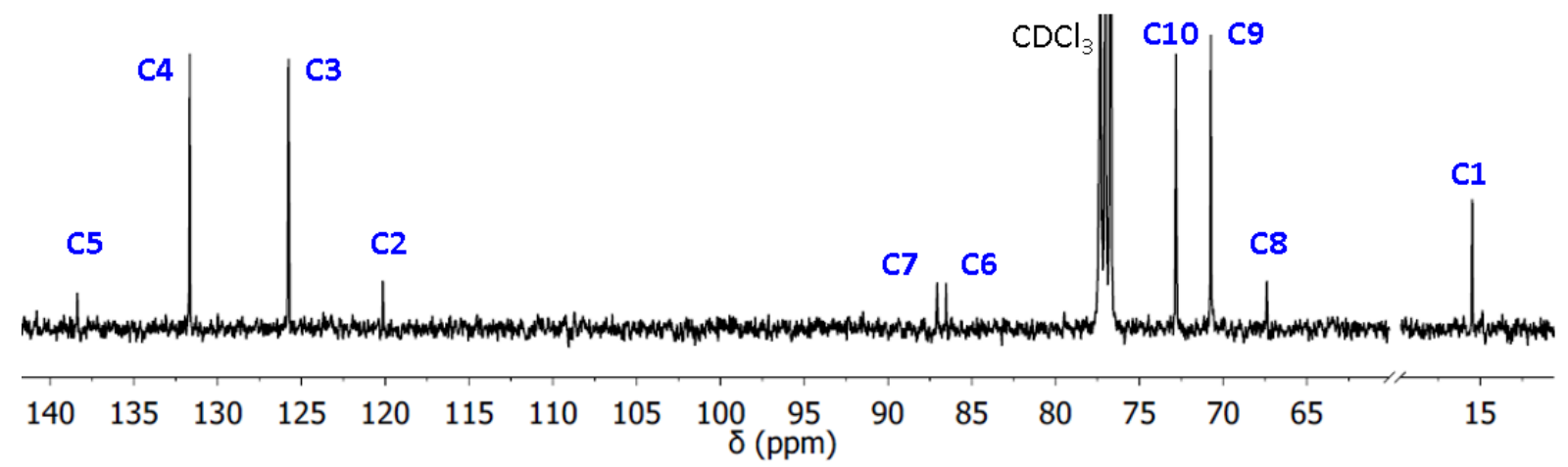

Figure S22: The ${ }^{13} \mathrm{C}\left\{{ }^{1} \mathrm{H}\right\}$ NMR spectrum of $\mathbf{3 c c}$ in $\mathrm{CDCl}_{3}$ 
1-(4-(Ethynyl)thioanisole)-1'-(3-(ethynyl)thioanisole)ferrocene: (3cd)

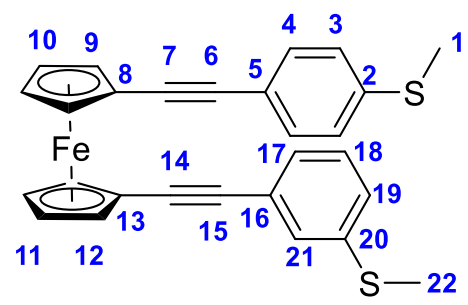

Following the general procedure, (2d) $(0.97 \mathrm{~g}, 2.14 \mathrm{mmol})$, Cul $(0.05 \mathrm{~g}, 0.24 \mathrm{mmol})$, 4ethynylthioanisole $(0.13 \mathrm{~g}, 0.89 \mathrm{mmol})$ and $\mathrm{Pd}\left(\mathrm{P}^{\mathrm{t}} \mathrm{Bu}_{3}\right)_{2}(0.02 \mathrm{~g}, 0.24 \mathrm{mmol})$ were added and the solution was left to stir overnight to form an orange precipitate. The solvent was removed in vacuo to give a black oil which was purified by chromatography on a silica column, eluted with n-hexane/DCM (1:0 $\rightarrow$ $3: 2 \mathrm{v} / \mathrm{v}$ ) to give the product as an orange solid $(0.35 \mathrm{~g}, 0.72 \mathrm{mmol}, 81 \%)$.

${ }^{1} \mathrm{H}$ NMR $\left(\mathrm{CDCl}_{3}, 298 \mathrm{~K}, 400 \mathrm{MHz}\right): \delta_{\mathrm{H}}=7.30\left(\mathrm{~d},{ }^{3} \mathrm{~J}_{\mathrm{H}-\mathrm{H}}=8.0 \mathrm{~Hz}, 2 \mathrm{H}, \mathrm{H4}\right), 7.28(\mathrm{~m}, 1 \mathrm{H}, \mathrm{H} 21), 7.16-7.14(\mathrm{~m}$, $3 \mathrm{H}, H 17-19), 7.10\left(\mathrm{~d},{ }^{3} \mathrm{~J}_{\mathrm{H}-\mathrm{H}}=8.0 \mathrm{~Hz}, 2 \mathrm{H}, H 3\right), 4.53(\mathrm{~m}, 4 \mathrm{H}, H 10 / 11), 4.31(\mathrm{~m}, 4 \mathrm{H}, H 9 / 12), 2.48(\mathrm{~s}, 3 \mathrm{H}$, H22), 2.45 (s, 3H, H1) ppm; ${ }^{13} \mathrm{C}\left\{{ }^{1} \mathrm{H}\right\}$ NMR $\left(\mathrm{CDCl}_{3}, 298 \mathrm{~K}, 100 \mathrm{MHz}\right): \delta_{\mathrm{c}}=138.8(\mathrm{C} 16), 138.6(\mathrm{C} 5), 131.8$ (C4), 129.0/128.7/128.2 (C17-19), 126.0 (C3), 125.5 (C21), 124.7 (C20), 120.3 (C2), 87.8 (C14), 87.2 (C7), 86.7 (C6), 86.5 (C15), 73.2/73.1 (C10/C11), 71.0/70.9 (C9/C12), 67.6 (C8), 67.2 (C13), 15.8 (C22), 15.7 (C1) ppm; IR: 2217 (-C=C-) $\mathrm{cm}^{-1}$; MS ES+: calcd. for $\mathrm{C}_{28} \mathrm{H}_{22} \mathrm{FeS}_{2}[\mathrm{M}]+478.0512$; found. 478.0496; EA calcd. C 70.29, H 4.63; found C 70.31, H 4.64.

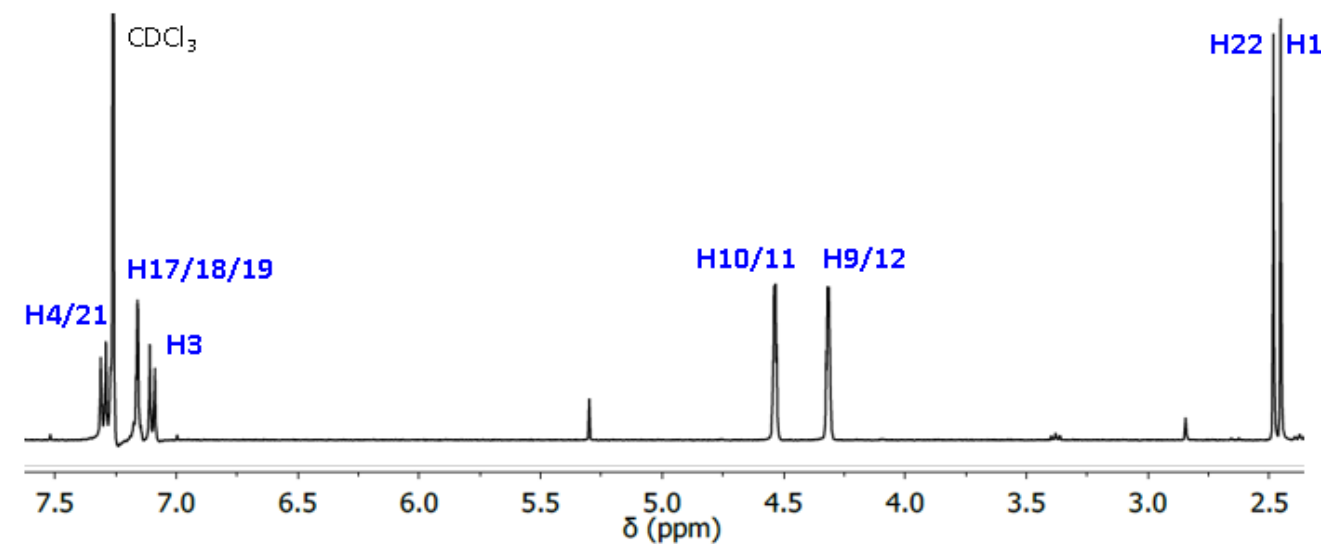

Figure S23: ${ }^{1} \mathrm{H}$ NMR spectrum of $3 \mathrm{~cd}$ in $\mathrm{CDCl}_{3}$

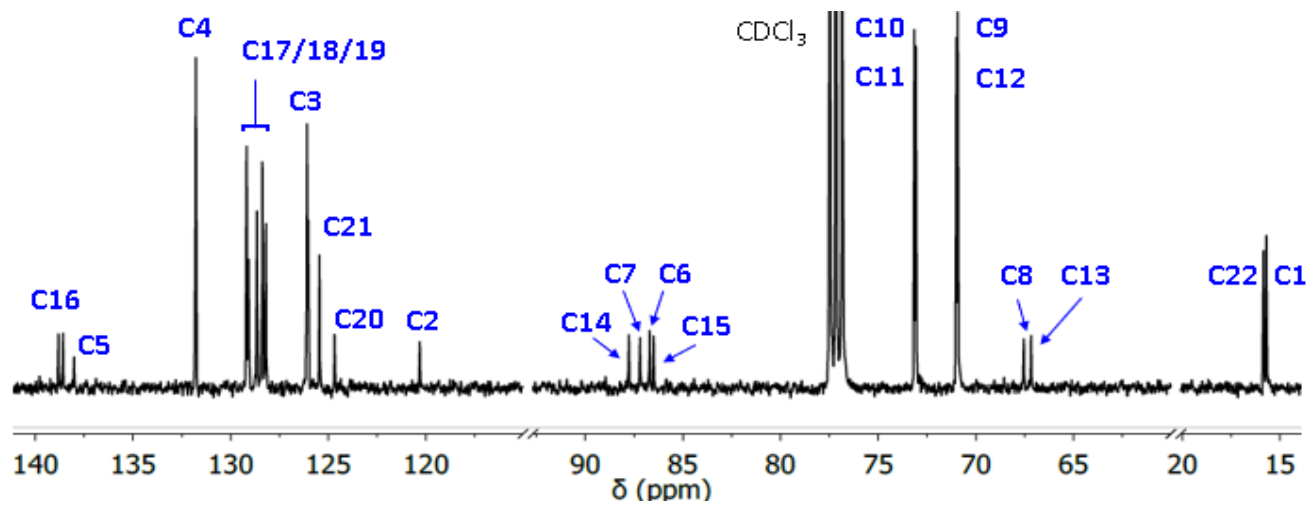

Figure S24: ${ }^{13} \mathrm{C}\left\{{ }^{1} \mathrm{H}\right\}$ NMR spectrum of $3 \mathrm{~cd}$ in $\mathrm{CDCl}_{3}$ 
1,1'-Di(3-(ethynyl)thioanisole)ferrocene: (3dd) $)^{2}$

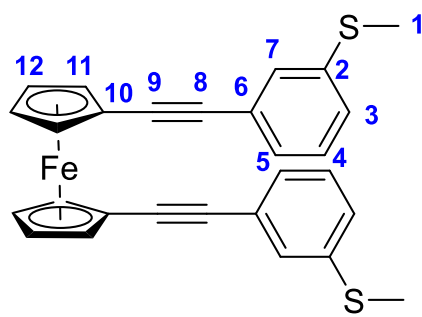

Following the general procedure, 1,1'-diiodoferrocene $(0.13 \mathrm{~g}, 0.31 \mathrm{mmol})$, Cul $(0.01 \mathrm{~g}, 0.02 \mathrm{mmol}), 3-$ ethynylthioanisole $(0.10 \mathrm{~g}, 0.68 \mathrm{mmol})$ and $\mathrm{Pd}\left(\mathrm{P}^{\mathrm{t}} \mathrm{Bu}_{3}\right)_{2}(0.01 \mathrm{~g}, 0.02 \mathrm{mmol})$ gave a brown oil which was purified by chromatography on a silica column, eluted with $n$-hexane/DCM $(1: 0 \rightarrow 3: 2 \mathrm{v} / \mathrm{v})$ to give the product as an orange solid ( $0.08 \mathrm{~g}, 0.17 \mathrm{mmol}, 62 \%)$.

${ }^{1} \mathrm{H}$ NMR $\left(\mathrm{CDCl}_{3}, 298 \mathrm{~K}, 400 \mathrm{MHz}\right): \delta_{\mathrm{H}}=7.30(\mathrm{br} \mathrm{s}, 2 \mathrm{H}), 7.22-7.16(\mathrm{~m}, 6 \mathrm{H}), 4.59\left(\mathrm{t},{ }^{3} \mathrm{~J}_{\mathrm{H}-\mathrm{H}}=2.0 \mathrm{~Hz}, 4 \mathrm{H}\right), 4.39$ $\left(\mathrm{t},{ }^{3} \mathrm{~J}_{\mathrm{H}-\mathrm{H}}=2.0 \mathrm{~Hz}, 4 \mathrm{H}\right), 2.49(\mathrm{~s}, 6 \mathrm{H}) \mathrm{ppm} ;{ }^{13} \mathrm{C}\left\{{ }^{1} \mathrm{H}\right\}$ NMR $\left(\mathrm{CDCl}_{3}, 298 \mathrm{~K}, 100 \mathrm{MHz}\right): \delta_{\mathrm{c}}=130.0(\mathrm{C} 6)$, 128.7/128.2/126.1 (C3-5), 124.6 (C7),118.4 (C2), 87.7 (C9), 86.5 (C8), 73.2 (C12), 71.1 (C11), 67.1 (C10), 15.9 (C1) ppm; IR: 2208 (-C $\equiv C-) \mathrm{cm}^{-1}$; MS ES+: calcd. for $\mathrm{C}_{28} \mathrm{H}_{22} \mathrm{FeS}_{2}$ [M]+ 478.0512; found. 478.0517; EA calcd. C 70.29, H 4.63; found C 70.15, H 4.53.

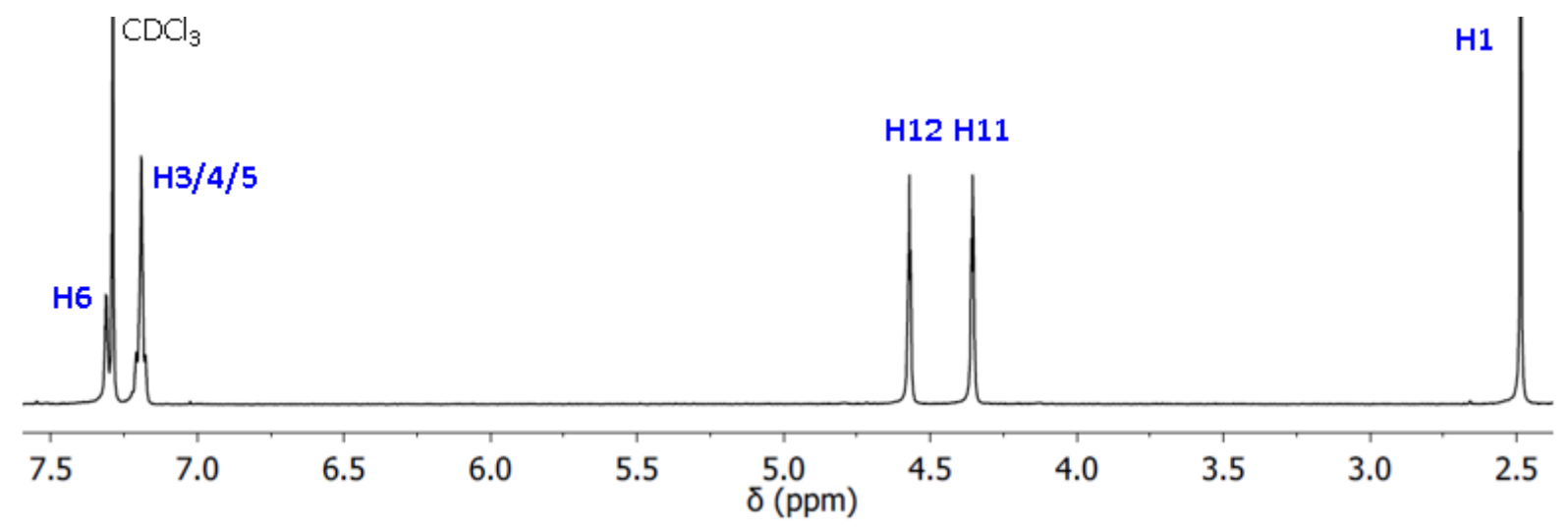

Figure S25: ${ }^{1} \mathrm{H}$ NMR spectrum of $3 \mathrm{dd}$ in $\mathrm{CDCl}_{3}$

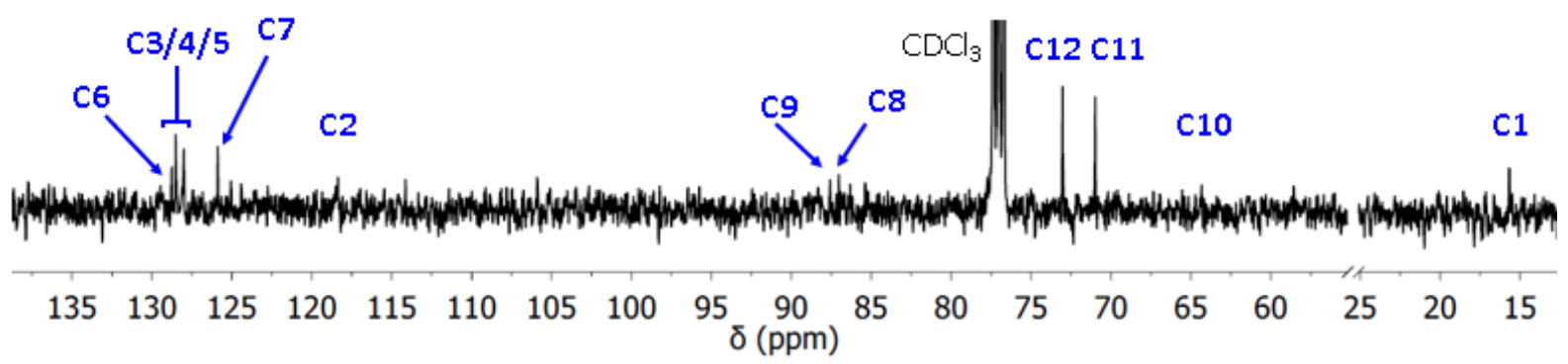

Figure S26: ${ }^{13} \mathrm{C}\left\{{ }^{1} \mathrm{H}\right\} \mathrm{NMR}$ spectrum of $\mathbf{3 d d}$ in $\mathrm{CDCl}_{3}$ 
(4-(Ethynyl)pyridine)ferrocene: (4a) ${ }^{3}$

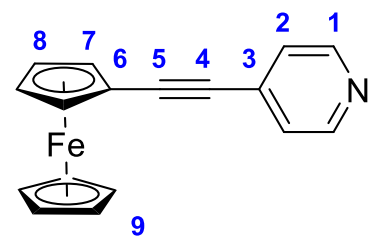

1-(Ethynyl)ferrocene $(0.50 \mathrm{~g}, 2.38 \mathrm{mmol})$, Cul $(0.02 \mathrm{~g}, 0.12 \mathrm{mmol}), \mathrm{Pd}\left(\mathrm{P}^{\mathrm{t}} \mathrm{Bu}_{3}\right)_{2}(0.06 \mathrm{~g}, 0.12 \mathrm{mmol})$ and 4-bromopyridine hydrochloride (1.46 g, $7.14 \mathrm{mmol})$, were dissolved in DIPA and stirred overnight to produce a brown precipitate. Removal of the solvent in vacuo gave a brown residue which was purified by recrystallization using boiling hexane to give the product as a deep red solid $(0.51 \mathrm{~g}, 1.73 \mathrm{mmol}, 73$ $\%)$.

${ }^{1} \mathrm{H}$ NMR $\left(\mathrm{CDCl}_{3}, 298 \mathrm{~K}, 400 \mathrm{MHz}\right): \delta_{\mathrm{H}}=8.59\left(\mathrm{dd},{ }^{3} \mathrm{~J}_{\mathrm{H}-\mathrm{H}}=4.4,{ }^{4} \mathrm{~J}_{\mathrm{H}-\mathrm{H}}=1.6 \mathrm{~Hz}, 2 \mathrm{H}, H 1\right), 7.35\left(\mathrm{dd},{ }^{3} \mathrm{H}_{\mathrm{H}-\mathrm{H}}=4.4\right.$, ${ }^{4} \mathrm{~J}_{\mathrm{H}-\mathrm{H}}=1.6 \mathrm{~Hz}, 2 \mathrm{H}, \mathrm{H2}$ ), 4.57 (pseudo-t, $\left.{ }^{3} \mathrm{~J}_{\mathrm{H}-\mathrm{H}}=2.0 \mathrm{~Hz}, 4 \mathrm{H}, H 7\right), 4.33\left(\mathrm{t},{ }^{3} \mathrm{~J}_{\mathrm{H}-\mathrm{H}}=2.0 \mathrm{~Hz}, 4 \mathrm{H}, \mathrm{H8}\right), 4.28(\mathrm{~s}, 5 \mathrm{H}$, H9) ppm; $\left.{ }^{13} \mathrm{C}^{1}{ }^{1} \mathrm{H}\right\}$ NMR (CDCl$\left., 298 \mathrm{~K}, 100 \mathrm{MHz}\right): \delta_{\mathrm{c}}=149.5$ (C1), 132.0 (C3), 125.5 (C2), 94.0 (C5), 83.5 (C4), 72.0 (C8), 70.0 (C9), 69.5 (C7), 63.5 (C6) ppm; IR: 2210 (-C=C-) cm-1; MS ES+: calcd. for $\mathrm{C}_{28} \mathrm{H}_{22} \mathrm{FeS}_{2}$ [M]+ 288.0476; found. 288.0471; EA calcd. C 71.11, H 4.56, N 4.88; found C 70.48, H 4.65, N 4.89.

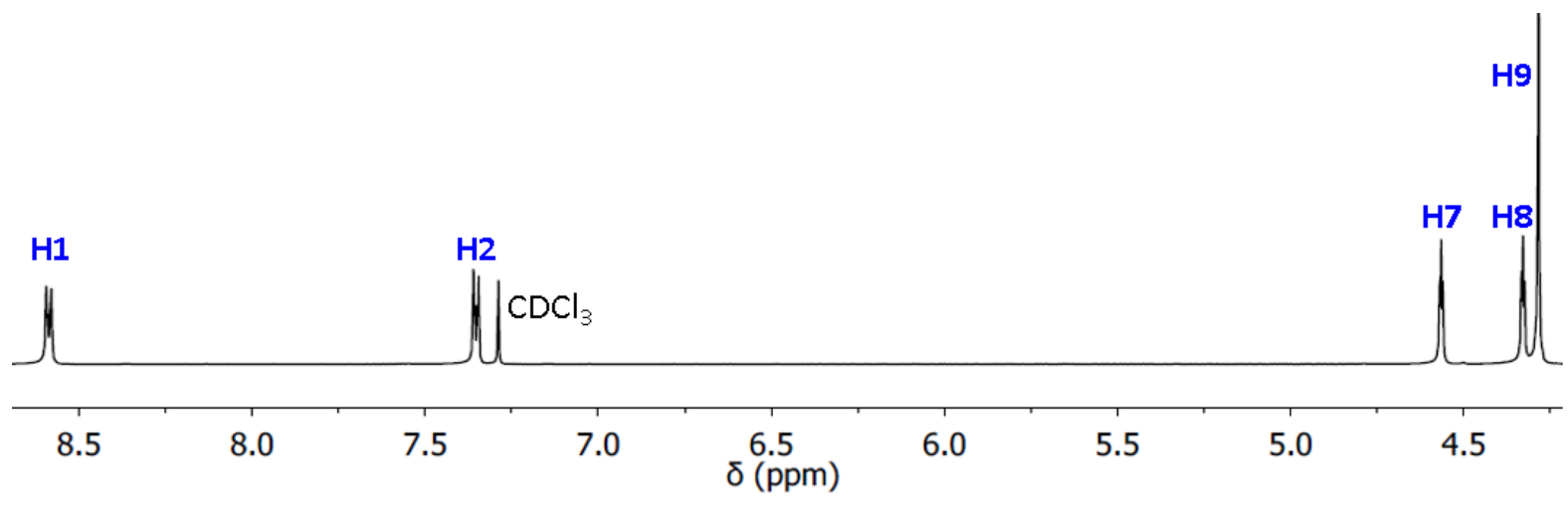

Figure S27: The ${ }^{1} \mathrm{H}$ NMR spectrum of 4 a in $\mathrm{CDCl}_{3}$

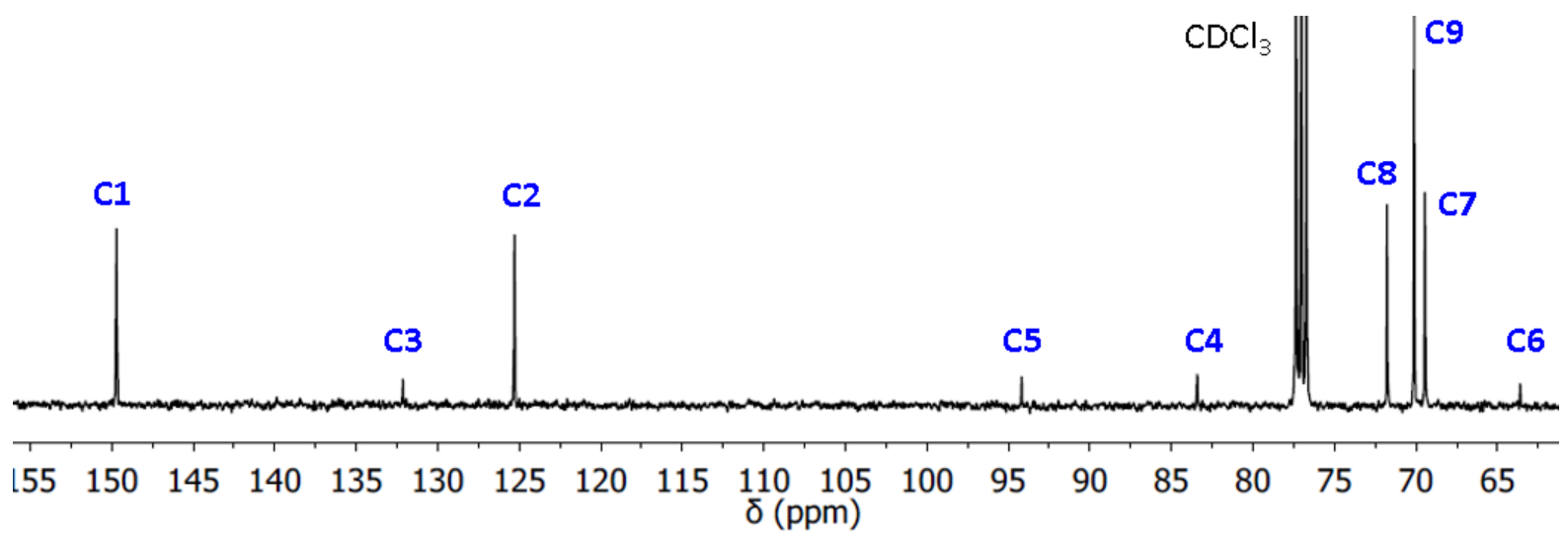

Figure S28: The ${ }^{13} \mathrm{C}\left\{{ }^{1} \mathrm{H}\right\}$ NMR spectrum of 4 a in $\mathrm{CDCl}_{3}$ 
(4-(Ethynyl)thioanisole)ferrocene: (4c) ${ }^{6}$

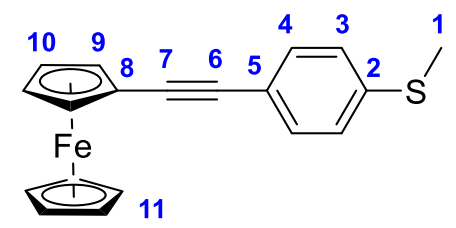

1-(Ethynyl)ferrocene $(0.25 \mathrm{~g}, 1.19 \mathrm{mmol})$, 4-bromothioanisole $(0.305 \mathrm{~g}, 1.5 \mathrm{mmol})$, Cul $(0.014 \mathrm{~g}, 0.075$

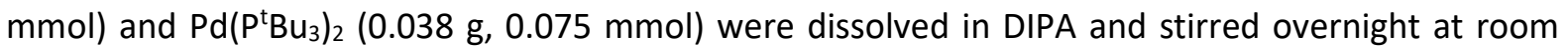
temperature to produce a tan precipitate. Removal of the solvent in vacuo gave a brown residue which was purified by chromatography on a silica column, eluted with petroleum spirit $\left(40-60^{\circ} \mathrm{C}\right) / \mathrm{DCM}(1: 1)$ to give the product as a yellow solid $(0.34 \mathrm{~g}, 1.02 \mathrm{mmol}, 86 \%)$.

${ }^{1} \mathrm{H}$ NMR $\left(\mathrm{CDCl}_{3}, 298 \mathrm{~K}, 400 \mathrm{MHz}\right): \delta_{\mathrm{H}}=7.42(\mathrm{~m}, 2 \mathrm{H}, \mathrm{H4}), 7.21(\mathrm{~m}, 2 \mathrm{H}, \mathrm{H3}), 4.52$ (pseudo-t, ${ }^{3} \mathrm{~J}_{\mathrm{H}-\mathrm{H}}=2.0 \mathrm{~Hz}$, $2 \mathrm{H}, H 9), 4.27(\mathrm{~s}, 5 \mathrm{H}, H 11), 4.26(\mathrm{~m}, 2 \mathrm{H}, H 10), 2.52(\mathrm{~s}, 3 \mathrm{H}, H 1) \mathrm{ppm} ;{ }^{13} \mathrm{C}\left\{{ }^{1} \mathrm{H}\right\} \mathrm{NMR}\left(\mathrm{CDCl}_{3}, 298 \mathrm{~K}, 100\right.$ $\mathrm{MHz}$ ): $\delta_{\mathrm{C}}=138.4(C 5), 131.7(C 4), 126.0(C 3), 120.3$ (C2), $88.4(C 7), 85.5$ (C6), 71.4 (C10), 70.0 (C11), 68.8 (C9), 65.3 (C8), 15.6 (C1) ppm; IR: 2210 (-C $\equiv C-)$ cm$^{-1}$; MS ES+: calcd. for $\mathrm{C}_{19} \mathrm{H}_{16} \mathrm{FeS}[\mathrm{M}]+332.0322$; found. 332.0314; EA calcd. C 68.69, H 4.85; found C 68.77, H 5.01.

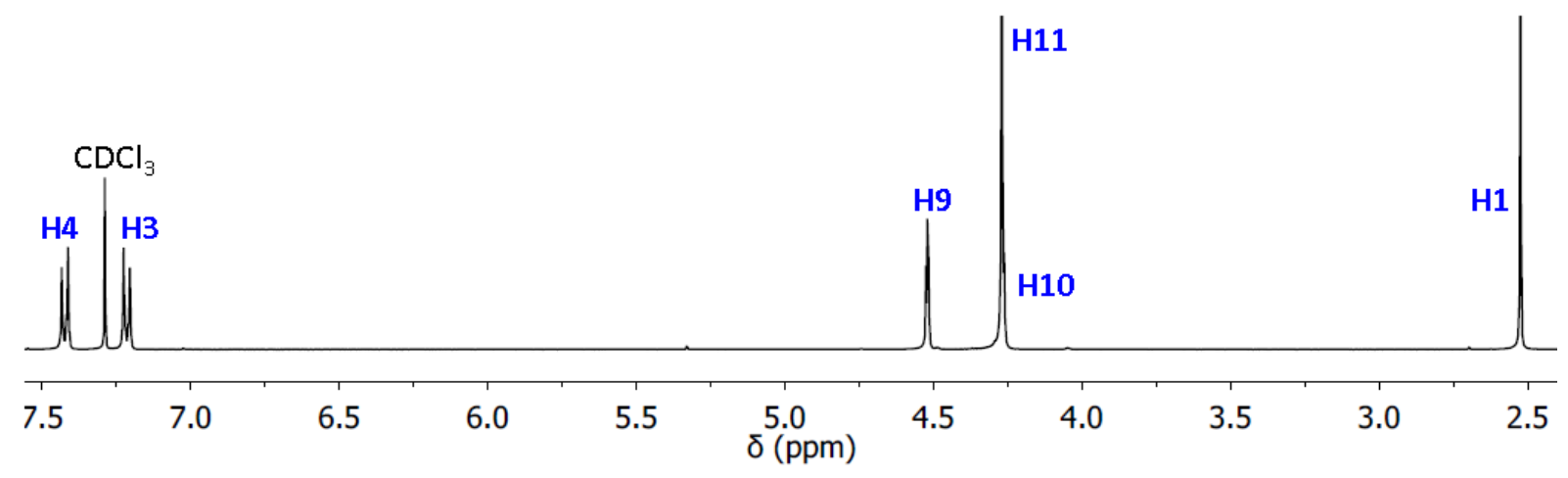

Figure S29: The ${ }^{1} \mathrm{H}$ NMR spectrum of $4 \mathrm{c}$ in $\mathrm{CDCl}_{3}$

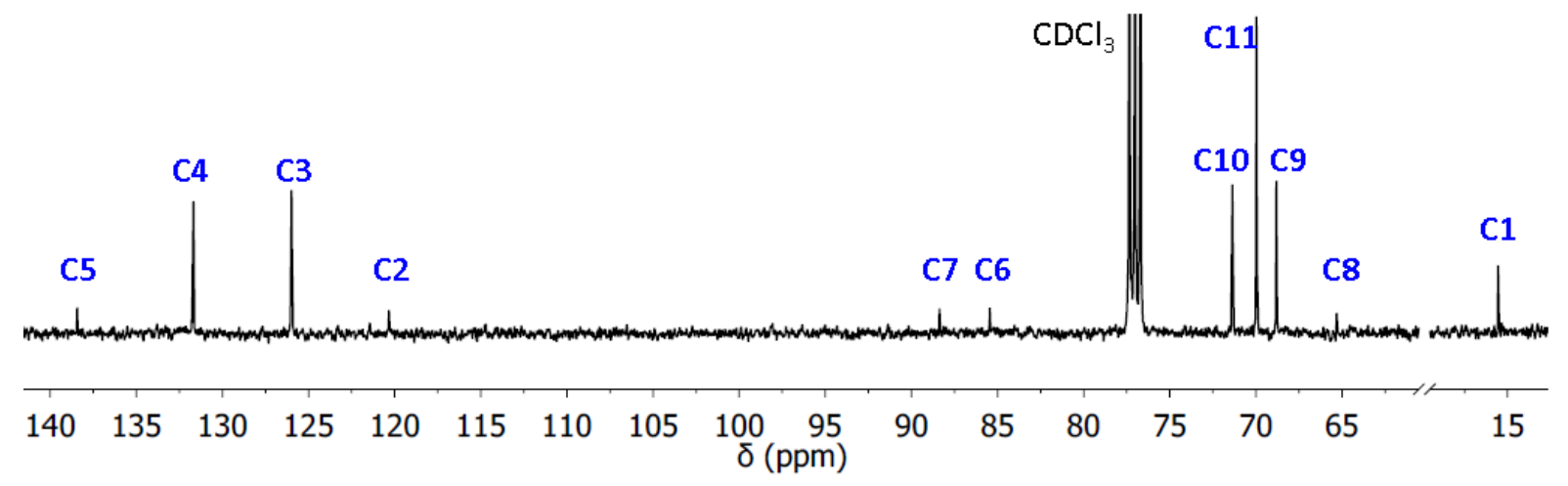

Figure S30: The ${ }^{13} \mathrm{C}\left\{{ }^{1} \mathrm{H}\right\}$ NMR spectrum of $4 \mathrm{c}$ in $\mathrm{CDCl}_{3}$ 
1-lodo-1'"-(4-(ethynyl)pyridine)biferrocene: (6a)

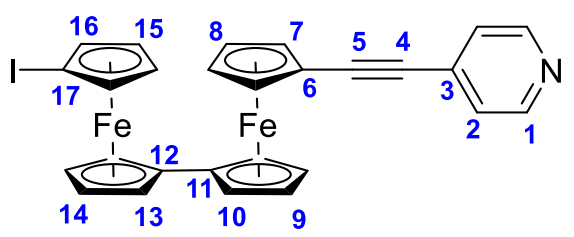

Following the general procedure, 1,1'"-diiodobiferrocene (1.78 g, $2.86 \mathrm{mmol}), \mathrm{Cul}(0.01 \mathrm{~g}, 0.07 \mathrm{mmol})$, 4-ethynylpyridine hydrochloride $(0.10 \mathrm{~g}, 0.72 \mathrm{mmol})$ and $\mathrm{Pd}\left(\mathrm{P}^{\mathrm{t} B \mathrm{Bu}_{3}}\right)_{2}(0.04 \mathrm{~g}, 0.07 \mathrm{mmol})$ gave a brown solid which was purified by chromatography on an alumina $\mathrm{V}$ column, eluted with $\mathrm{n}$-hexane/ethyl acetate $(1: 0 \rightarrow 7: 3 \mathrm{v} / \mathrm{v})$ to give the product as a red solid $(0.12 \mathrm{~g}, 0.20 \mathrm{mmol}, 28 \%)$.

${ }^{1} \mathrm{H} \mathrm{NMR}\left(\mathrm{CDCl}_{3}, 298 \mathrm{~K}, 400 \mathrm{MHz}\right): \delta_{\mathrm{H}}=8.56(\mathrm{br} \mathrm{s}, 2 \mathrm{H}, \mathrm{H} 2), 7.25\left(\mathrm{dd},{ }^{3} \mathrm{~J}_{\mathrm{H}-\mathrm{H}}=4.4,{ }^{4} J_{\mathrm{H}-\mathrm{H}}=1.6 \mathrm{~Hz}, 2 \mathrm{H}, \mathrm{H} 1\right)$, 4.46 (pseudo-t, ${ }^{3} J_{\mathrm{H}-\mathrm{H}}=2.0 \mathrm{~Hz}, 2 \mathrm{H}, \mathrm{H10}$ ), 4.32 (pseudo-t, ${ }^{3} \mathrm{~J}_{\mathrm{H}-\mathrm{H}}=2.0 \mathrm{~Hz}, 2 \mathrm{H}, H 8$ ), 4.31 (pseudo-t, ${ }^{3} \mathrm{~J}_{\mathrm{H}-\mathrm{H}}=$ $2.0 \mathrm{~Hz}, 2 \mathrm{H}, H 14$ ), 4.30 (pseudo-t, ${ }^{3} \mathrm{~J}_{\mathrm{H}-\mathrm{H}}=2.0 \mathrm{~Hz}, 2 \mathrm{H}, H 9$ ), 4.15 (pseudo-t, ${ }^{3} \mathrm{~J}_{\mathrm{H}-\mathrm{H}}=2.0 \mathrm{~Hz}, 4 \mathrm{H}, H 7 / \mathrm{H} 13$ ), 4.14 (pseudo-t, ${ }^{3} \mathrm{~J}_{\mathrm{H}-\mathrm{H}}=2.0 \mathrm{~Hz}, 2 \mathrm{H}, H 15$ ), 3.97 (pseudo-t, $\left.{ }^{3} \mathrm{~J}_{\mathrm{H}-\mathrm{H}}=2.0 \mathrm{~Hz}, 2 \mathrm{H}, \mathrm{H} 16\right)$ ppm; ${ }^{13} \mathrm{C}\left\{{ }^{1} \mathrm{H}\right\} \mathrm{NMR}$ $\left(\mathrm{CDCl}_{3}, 298 \mathrm{~K}, 100 \mathrm{MHz}\right): \delta_{\mathrm{C}}=149.6(C 2), 132.3(C 3), 125.3(C 1), 94.0(C 5), 84.7(C 11), 84.2(C 13), 83.8$ (C4), 75.8 (C15), 73.0 (C8), 70.9 (C7/C13), 70.5 (C7/C13), 70.0 (C16), 69.8 (C9/C14), 69.5 (C9/C14), 68.3 (C10), 64.6 (C6), 40.8 (C17) ppm; IR: 2209 (-C $\equiv \mathrm{C}-) \mathrm{cm}^{-1}$; MS ES+: calcd. for $\mathrm{C}_{27} \mathrm{H}_{21} \mathrm{Fe}_{2} \mathrm{NI}$ [M]+ 597.9418; found. 597.9423.

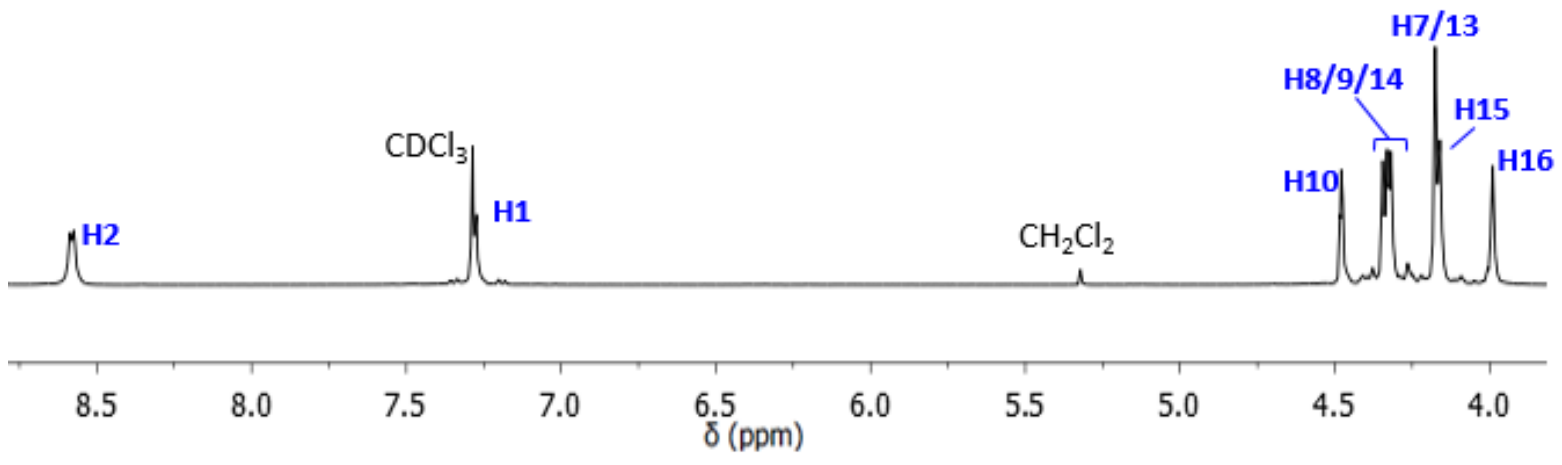

Figure S31: The ${ }^{1} \mathrm{H}$ NMR spectrum of 6 a in $\mathrm{CDCl}_{3}$
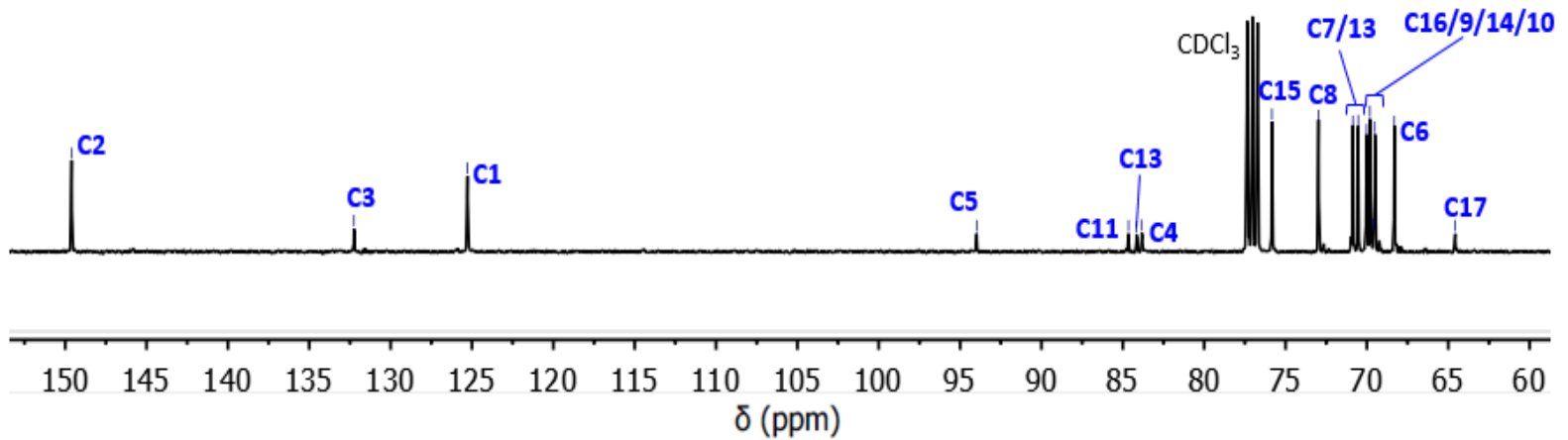

Figure S32: The ${ }^{13} \mathrm{C}\left\{{ }^{1} \mathrm{H}\right\}$ NMR spectrum of 6 a in $\mathrm{CDCl}_{3}$ 
1-lodo-1"'-(4-(ethynyl)thioanisole)biferrocene: (6c)

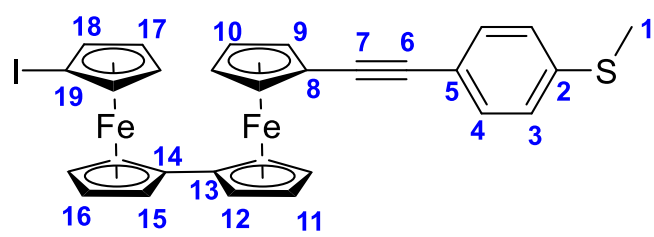

Following the general procedure, 1,1'"'-diiodobiferrocene $(1.68 \mathrm{~g}, 2.68 \mathrm{mmol})$, Cul $(0.01 \mathrm{~g}, 0.07 \mathrm{mmol})$, 4-ethynylthioanisole $(0.10 \mathrm{~g}, 0.67 \mathrm{mmol})$ and $\mathrm{Pd}\left(\mathrm{P}^{\mathrm{t}} \mathrm{Bu}_{3}\right)_{2}(0.03 \mathrm{~g}, 0.07 \mathrm{mmol})$ gave a brown solid which was purified by chromatography on a silica column, eluted with $\mathrm{n}$-hexane/DCM (1:0 $\rightarrow$ 7:3 v/v) to give the product as an orange solid $(0.15 \mathrm{~g}, 0.23 \mathrm{mmol}, 30 \%)$.

${ }^{1} \mathrm{H}$ NMR $\left(\mathrm{CDCl}_{3}, 298 \mathrm{~K}, 400 \mathrm{MHz}\right): \delta_{\mathrm{H}}=7.36\left(\mathrm{~d},{ }^{3} \mathrm{~J}_{\mathrm{H}-\mathrm{H}}=8.4 \mathrm{~Hz}, 2 \mathrm{H}, \mathrm{H3}\right), 7.19\left(\mathrm{~d},{ }^{3} \mathrm{~J}_{\mathrm{H}-\mathrm{H}}=8.4 \mathrm{~Hz}, 2 \mathrm{H}, \mathrm{H4}\right)$, 4.43 (pseudo-t, ${ }^{3} J_{\mathrm{H}-\mathrm{H}}=2.0 \mathrm{~Hz}, 2 \mathrm{H}, H 11$ ), 4.34 (pseudo-t, ${ }^{3} \mathrm{~J}_{\mathrm{H}-\mathrm{H}}=2.0 \mathrm{~Hz}, 2 \mathrm{H}, H 16$ ), 4.28 (pseudo-t, ${ }^{3} \mathrm{~J}_{\mathrm{H}-\mathrm{H}}=$ $2.0 \mathrm{~Hz}, 2 \mathrm{H}, H 12$ ), 4.26 (pseudo-t, ${ }^{3} \mathrm{~J}_{\mathrm{H}-\mathrm{H}}=2.0 \mathrm{~Hz}, 2 \mathrm{H}, H 10$ ), 4.17 (pseudo-t, ${ }^{3} \mathrm{~J}_{\mathrm{H}-\mathrm{H}}=2.0 \mathrm{~Hz}, 2 \mathrm{H}, H 15$ ), 4.15 (pseudo-t, ${ }^{3} \mathrm{~J}_{\mathrm{H}-\mathrm{H}}=2.0 \mathrm{~Hz}, 2 \mathrm{H}, \mathrm{H17}$ ), 4.08 (pseudo-t, ${ }^{3} \mathrm{~J}_{\mathrm{H}-\mathrm{H}}=2.0 \mathrm{~Hz}, 2 \mathrm{H}, H 9$ ), 3.97 (pseudo-t, ${ }^{3} \mathrm{~J}_{\mathrm{H}-\mathrm{H}}=2.0 \mathrm{~Hz}$, $2 \mathrm{H}, H 18), 2.51(\mathrm{~s}, 3 \mathrm{H}, \mathrm{H1}) \mathrm{ppm} ;{ }^{13} \mathrm{C}\left\{{ }^{1} \mathrm{H}\right\} \mathrm{NMR}\left(\mathrm{CDCl}_{3}, 298 \mathrm{~K}, 100 \mathrm{MHz}\right): \delta_{\mathrm{c}}=138.4(\mathrm{C2}), 131.8(\mathrm{C3}), 126.1$ (C4), 120.6 (C5), 88.3 (C7), 85.9 (C6), 84.9 (C14), 84.3 (C13), 75.9 (C17), 72.8 (C10), 71.0 (C15), 70.2

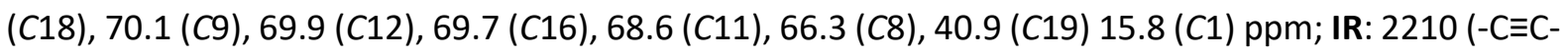
) $\mathrm{cm}^{-1}$; MS ES+: calcd. for $\mathrm{C}_{29} \mathrm{H}_{24} \mathrm{Fe}_{2} \mathrm{SI}[\mathrm{M}]+642.9337$; found. 642.9327.

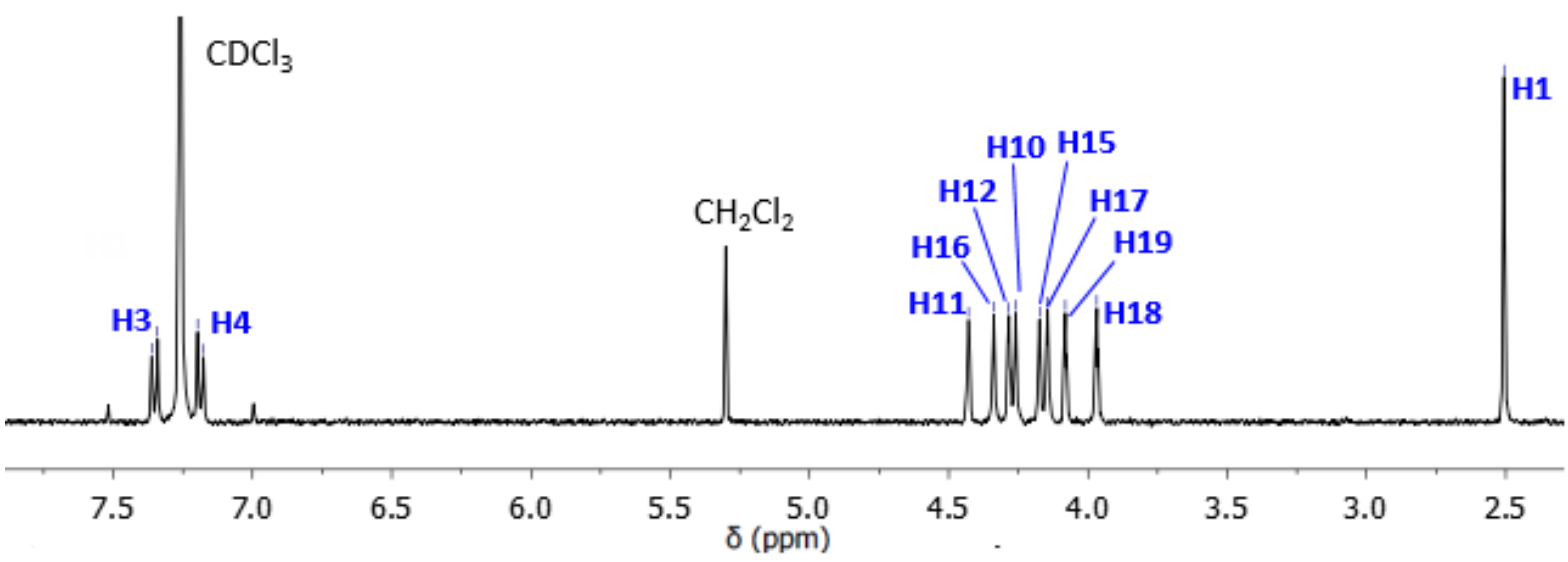

Figure S33: The ${ }^{1} \mathrm{H}$ NMR spectrum of $6 \mathrm{c}$ in $\mathrm{CDCl}_{3}$

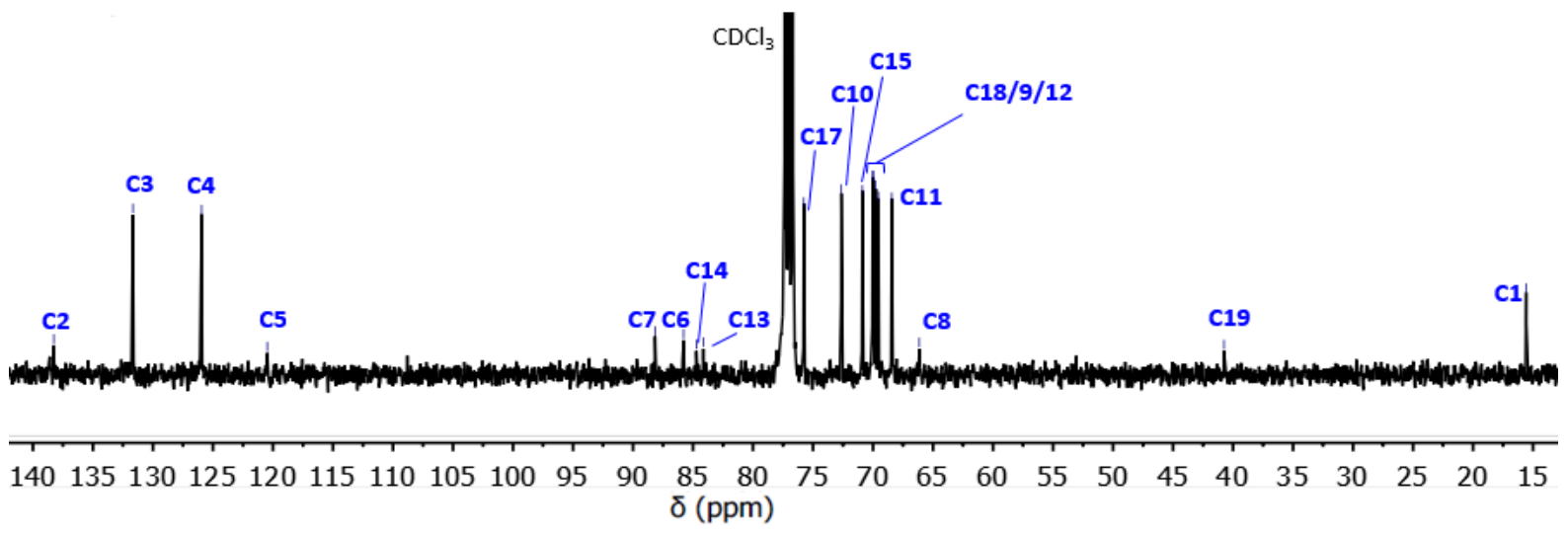

Figure S34: The ${ }^{13} \mathrm{C}\left\{{ }^{1} \mathrm{H}\right\}$ NMR spectrum of $6 \mathrm{c}$ in $\mathrm{CDCl}_{3}$ 
1,1'"-Di(4-(ethynyl)pyridine)biferrocene: (7aa)

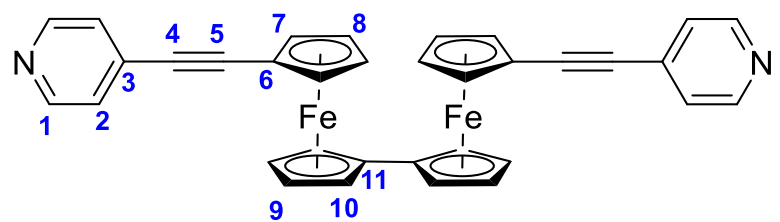

Following the general procedure, 1,1'"-diiodobiferrocene $(0.20 \mathrm{~g}, 0.32 \mathrm{mmol}), \mathrm{Cul}(0.01 \mathrm{~g}, 0.03 \mathrm{mmol})$, 4-ethynylpyridine hydrochloride $(0.18 \mathrm{~g}, 1.28 \mathrm{mmol})$ and $\mathrm{Pd}\left(\mathrm{P}^{\mathrm{t}} \mathrm{Bu}_{3}\right)_{2}(0.02 \mathrm{~g}, 0.03 \mathrm{mmol})$ gave a brown solid which was purified by chromatography on an alumina $V$ column, eluted with $n$-hexane/ethyl acetate $(1: 0 \rightarrow 0: 1 \mathrm{v} / \mathrm{v})$ to give the product as an orange solid $(0.11 \mathrm{~g}, 0.19 \mathrm{mmol}, 59 \%)$.

${ }^{1} \mathrm{H}$ NMR $\left(\mathrm{CDCl}_{3}, 298 \mathrm{~K}, 400 \mathrm{MHz}\right): \delta_{\mathrm{H}}=8.54\left(\mathrm{~d},{ }^{3} \mathrm{~J}_{\mathrm{H}-\mathrm{H}}=5.2 \mathrm{~Hz}, 4 \mathrm{H}, \mathrm{H2}\right), 7.22\left(\mathrm{~d},{ }^{3} \mathrm{~J}_{\mathrm{H}-\mathrm{H}}=5.2 \mathrm{~Hz}, 4 \mathrm{H}, \mathrm{H} 1\right)$, 4.41 (pseudo-t, ${ }^{3} \mathrm{H}_{\mathrm{H}-\mathrm{H}}=2.0 \mathrm{~Hz}, 4 \mathrm{H}, \mathrm{H10}$ ), 4.30 (pseudo-t, $\left.{ }^{3} \mathrm{~J}_{\mathrm{H}-\mathrm{H}}=2.0 \mathrm{~Hz}, 4 \mathrm{H}, H 8\right), 4.20$ (pseudo-t, ${ }^{3} \mathrm{~J}_{\mathrm{H}-\mathrm{H}}=$ $2.0 \mathrm{~Hz}, 4 \mathrm{H}, H 9$ ), 4.13 (pseudo-t, $\left.{ }^{3} \mathrm{H}_{\mathrm{H}-\mathrm{H}}=2.0 \mathrm{~Hz}, 4 \mathrm{H}, H 7\right) \mathrm{ppm} ;{ }^{13} \mathrm{C}\left\{{ }^{1} \mathrm{H}\right\} \mathrm{NMR}\left(\mathrm{CDCl}_{3}, 298 \mathrm{~K}, 100 \mathrm{MHz}\right): \delta_{\mathrm{c}}=$ 149.7 (C2), 132.4 (C3), 125.4 (C1), 94.1 (C5), 84.4 (C11), 84.0 (C4), 73.1 (C8), 70.6 (C7), 69.8 (C9), 68.2 (C10), 64.8 (C6) ppm; IR: 2213 (-C $\equiv C-) \mathrm{cm}^{-1}$; MS ES+: calcd. for $\mathrm{C}_{34} \mathrm{H}_{25} \mathrm{Fe}_{2} \mathrm{~N}_{2}$ [M]+ 573.0717; found. 573.0711 .

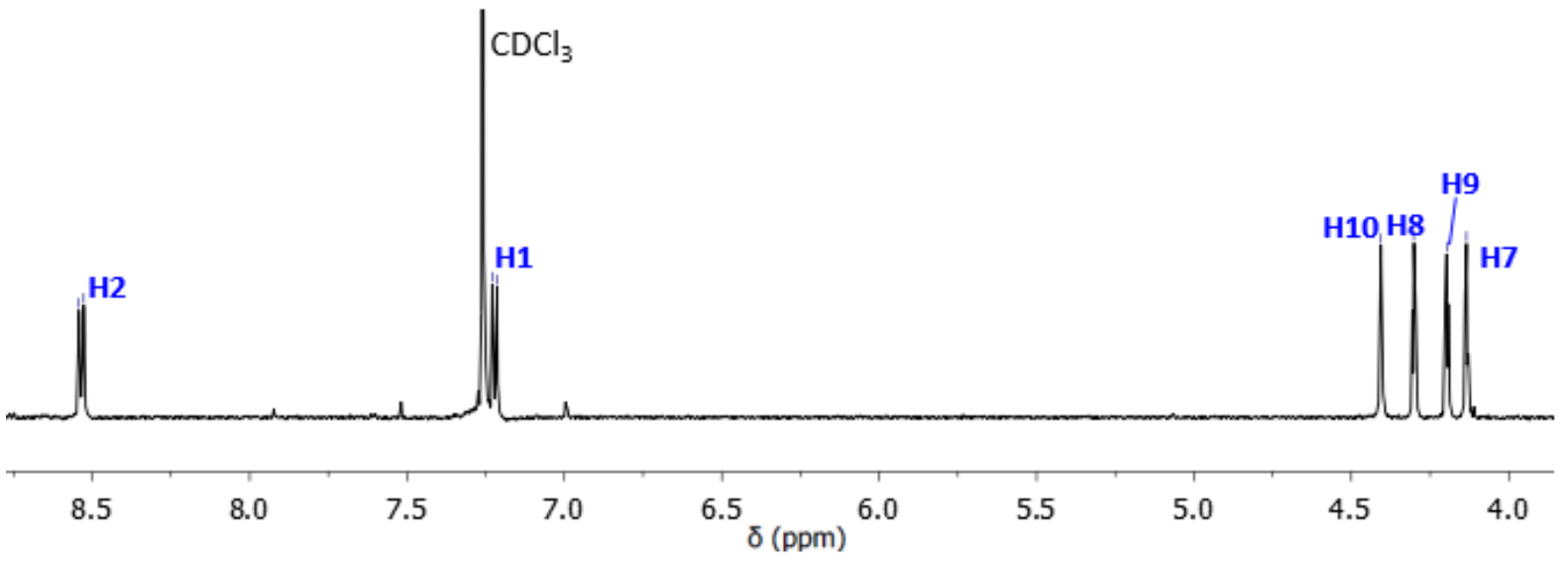

Figure S35: The ${ }^{1} \mathrm{H}$ NMR spectrum of 7 aa in $\mathrm{CDCl}_{3}$

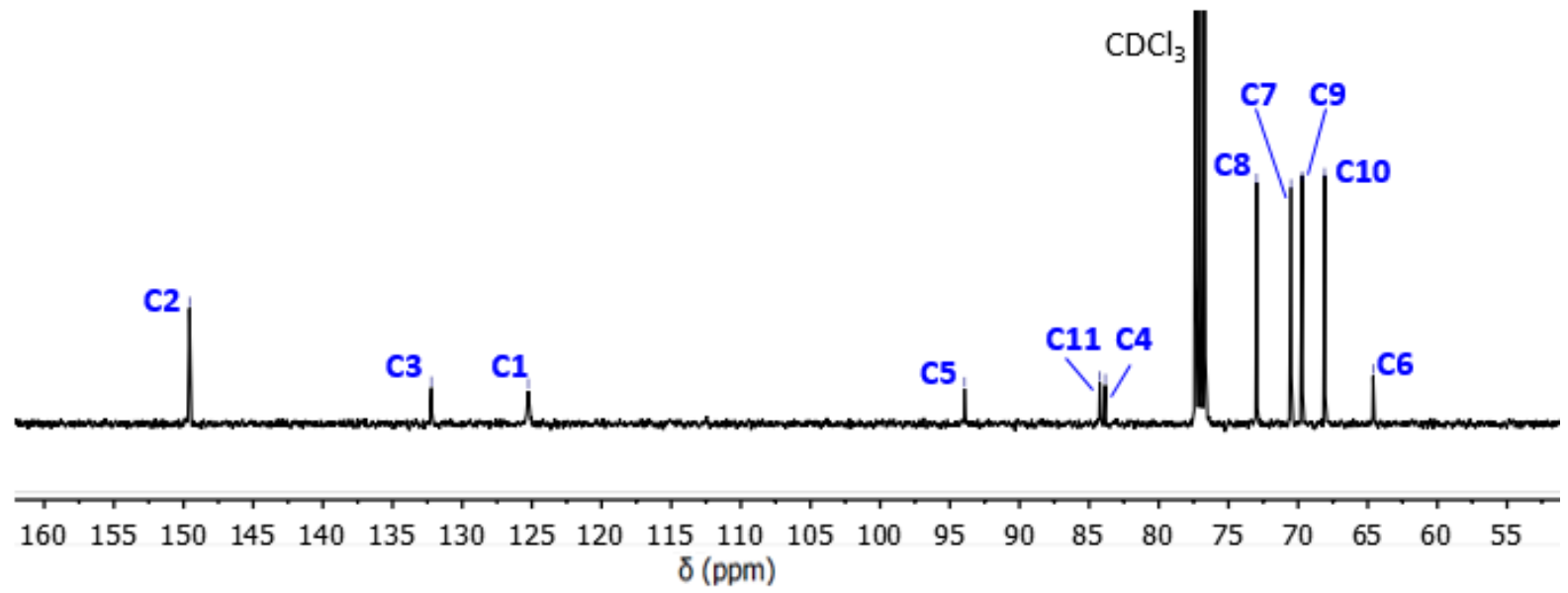

Figure S36: The ${ }^{13} \mathrm{C}\left\{{ }^{1} \mathrm{H}\right\}$ NMR spectrum of 7aa in $\mathrm{CDCl}_{3}$ 
1-(4-(Ethynyl)pyridine)-1"'-(3-(ethynyl)pyridine)biferrocene: (7ab)

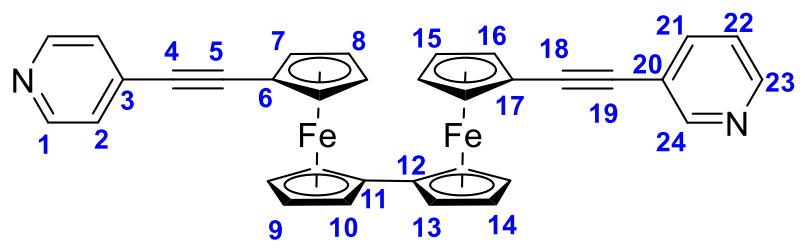

Following the general procedure, $(6 \mathrm{a})(0.10 \mathrm{~g}, 0.17 \mathrm{mmol}), \mathrm{Cul}(0.01 \mathrm{~g}, 0.02 \mathrm{mmol})$, 3-ethynylpyridine $(0.04 \mathrm{~g}, 0.33 \mathrm{mmol})$ and $\mathrm{Pd}\left(\mathrm{P}^{\mathrm{t}} \mathrm{Bu}_{3}\right)_{2}(0.01 \mathrm{~g}, 0.02 \mathrm{mmol})$ gave a brown solid which was purified by chromatography on an alumina $V$ column, eluted with $n$-hexane/ethyl acetate $(1: 0 \rightarrow 0: 1 \mathrm{v} / \mathrm{v})$ to give the product as an orange solid $(0.04 \mathrm{~g}, 0.07 \mathrm{mmol}, 44 \%)$.

${ }^{1} \mathrm{H}$ NMR $\left(\mathrm{CDCl}_{3}, 298 \mathrm{~K}, 400 \mathrm{MHz}\right): \delta_{\mathrm{H}}=8.63(\mathrm{~s}, 1 \mathrm{H}, \mathrm{H} 24), 8.54\left(\mathrm{~d},{ }^{3} \mathrm{~J}_{\mathrm{H}-\mathrm{H}}=4.4 \mathrm{~Hz}, 2 \mathrm{H}, \mathrm{H} 2\right), 8.52\left(\mathrm{~d},{ }^{3} \mathrm{H}_{\mathrm{H}-\mathrm{H}}=\right.$ $4.0 \mathrm{~Hz}, 1 \mathrm{H}, H 23), 7.65\left(\mathrm{~d},{ }^{3} J_{\mathrm{H}-\mathrm{H}}=8.4 \mathrm{~Hz}, 1 \mathrm{H}, \mathrm{H} 21\right), 7.24-7.20(\mathrm{~m}, 3 \mathrm{H}, \mathrm{H} 1 / \mathrm{H} 22), 4.42$ (pseudo-t, ${ }^{3} \mathrm{~J}_{\mathrm{H}-\mathrm{H}}=2.0$ $\mathrm{Hz}, 2 \mathrm{H}, H 10 / H 14$ ), 4.40 (pseudo-t, ${ }^{3} J_{\mathrm{H}-\mathrm{H}}=2.0 \mathrm{~Hz}, 2 \mathrm{H}, H 10 / H 14$ ), 4.30 (pseudo-t, ${ }^{3} \mathrm{H}_{\mathrm{H}-\mathrm{H}}=2.0 \mathrm{~Hz}, 2 \mathrm{H}, H 7$ ), 4.28 (pseudo-t, ${ }^{3} \mathrm{~J}_{\mathrm{H}-\mathrm{H}}=2.0 \mathrm{~Hz}, 2 \mathrm{H}, H 15$ ), 4.20 (pseudo-t, ${ }^{3} \mathrm{~J}_{\mathrm{H}-\mathrm{H}}=2.0 \mathrm{~Hz}, 4 \mathrm{H}, H 9 / H 13$ ), 4.13 (pseudo-t, ${ }^{3} \mathrm{~J}_{\mathrm{H}-}$ $\mathrm{H}=2.0 \mathrm{~Hz}, 2 \mathrm{H}, \mathrm{H8}$ ), 4.11 (pseudo-t, $\left.{ }^{3} \mathrm{~J}_{\mathrm{H}-\mathrm{H}}=2.0 \mathrm{~Hz}, 2 \mathrm{H}, \mathrm{H16}\right) \mathrm{ppm} ;{ }^{13} \mathrm{C}\left\{{ }^{1} \mathrm{H}\right\}$ NMR $\left(\mathrm{CDCl}_{3}, 298 \mathrm{~K}, 100 \mathrm{MHz}\right)$ : $\delta_{c}=152.1$ (C24), 149.7 (C1), 147.9 (C23), 138.1 (C21), 132.4 (C3), 125.4 (C2), 123.1 (C22), 121.4 (C20), 94.2 (C5), 92.0 (C18), 84.5 (C11/C12), 84.2 (C11/C12), 83.9 (C4), 82.9 (C19), 73.1 (C7/C15), 72.9 (C7/C15), 70.7 (C8), 70.3 (C16), 69.8 (C9/C13), 69.8 (C9/C13), 68.3 (C10/C14), 68.2 (C10/C14), 65.4 (C17), 64.7 (C6) ppm; IR: 2205 (-CEC-) cm-1; MS ES+: calcd. for $\mathrm{C}_{34} \mathrm{H}_{25} \mathrm{Fe}_{2} \mathrm{~N}_{2}$ [M]+ 573.0717; found. 573.0703 .

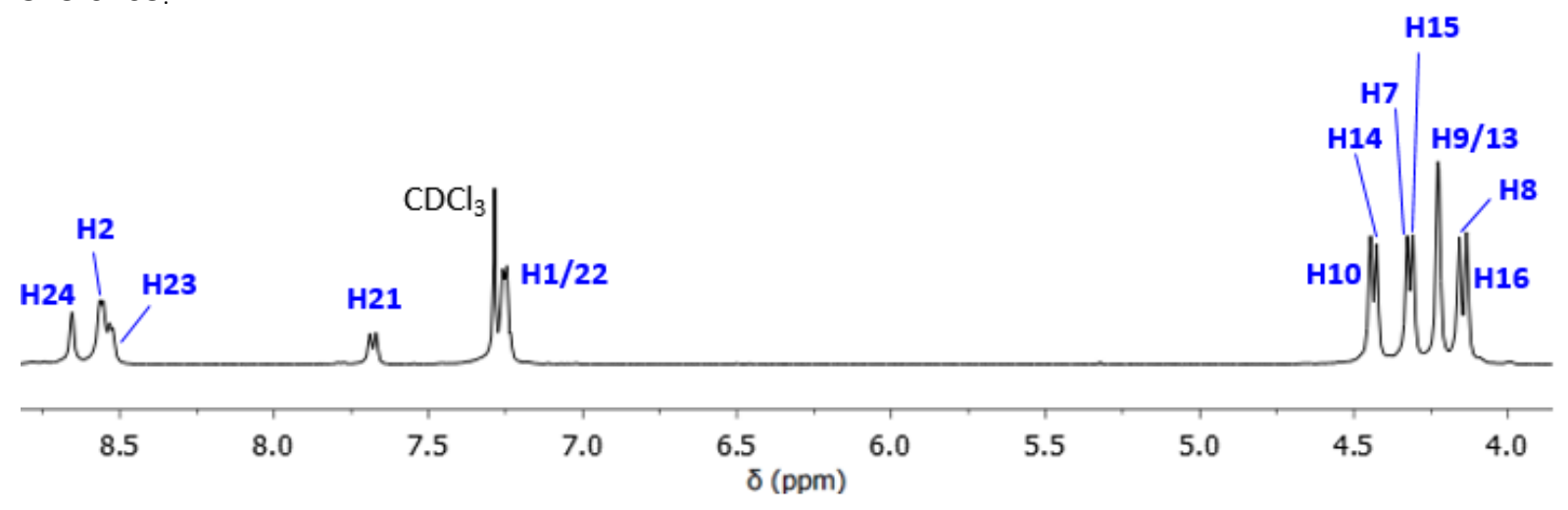

Figure S37: The ${ }^{1} \mathrm{H}$ NMR spectrum of $7 \mathrm{ab}$ in $\mathrm{CDCl}_{3}$

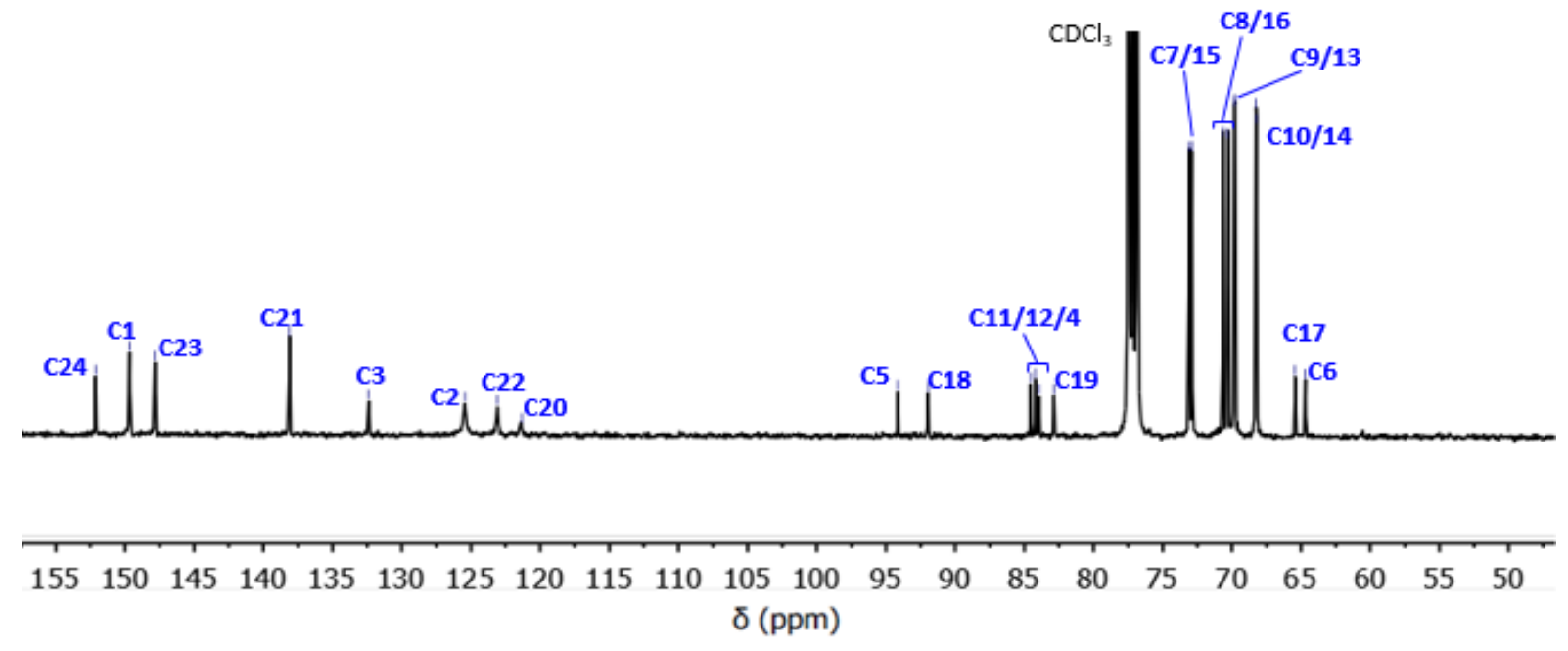

Figure S38: The ${ }^{13} \mathrm{C}\left\{{ }^{1} \mathrm{H}\right\}$ NMR spectrum of $7 \mathrm{ab}$ in $\mathrm{CDCl}_{3}$ 
1-(4-(Ethyny))pyridine)-1"'-(4-(ethynyl)thioanisole)biferrocene: (7ac)

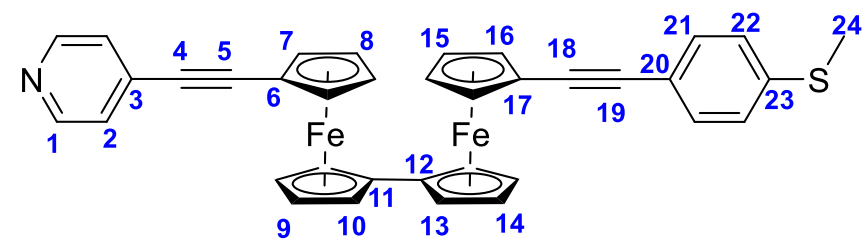

Following the general procedure, $(6 \mathrm{a})(0.05 \mathrm{~g}, 0.08 \mathrm{mmol})$, Cul $(0.01 \mathrm{~g}, 0.01 \mathrm{mmol})$, 4ethynylthioanisole $(0.02 \mathrm{~g}, 0.16 \mathrm{mmol})$ and $\mathrm{Pd}\left(\mathrm{P}^{\mathrm{t}} \mathrm{Bu}_{3}\right)_{2}(0.01 \mathrm{~g}, 0.01 \mathrm{mmol})$ gave a brown solid which was purified by chromatography on an alumina $V$ column, eluted with $n$-hexane/ethyl acetate $(1: 0 \rightarrow$ $7: 3 \mathrm{v} / \mathrm{v})$ to give the product as an orange solid $(0.04 \mathrm{~g}, 0.06 \mathrm{mmol}, 73 \%)$.

${ }^{1} \mathrm{H}$ NMR $\left(\mathrm{CDCl}_{3}, 298 \mathrm{~K}, 400 \mathrm{MHz}\right): \delta_{\mathrm{H}}=8.54(\mathrm{br} \mathrm{s}, 2 \mathrm{H}, \mathrm{H2}), 7.32\left(\mathrm{~d},{ }^{3} \mathrm{~J}_{\mathrm{H}-\mathrm{H}}=8.4 \mathrm{~Hz}, 2 \mathrm{H}, \mathrm{H} 21\right), 7.23\left(\mathrm{~d},{ }^{3} \mathrm{~J}_{\mathrm{H}-}\right.$ $\mathrm{H}=5.4 \mathrm{~Hz}, 2 \mathrm{H}, H 1), 7.17\left(\mathrm{~d},{ }^{3} J_{\mathrm{H}-\mathrm{H}}=8.4 \mathrm{~Hz}, 2 \mathrm{H}, H 22\right), 4.44$ (pseudo-t, ${ }^{3} \mathrm{~J}_{\mathrm{H}-\mathrm{H}}=2.0 \mathrm{~Hz}, 2 \mathrm{H}, H 14$ ), 4.38 (pseudo-t, ${ }^{3} \mathrm{H}_{\mathrm{H}-\mathrm{H}}=2.0 \mathrm{~Hz}, 2 \mathrm{H}, H 10$ ), 4.31 (pseudo-t, ${ }^{3} \mathrm{~J}_{\mathrm{H}-\mathrm{H}}=2.0 \mathrm{~Hz}, 2 \mathrm{H}, \mathrm{H8}$ ), 4.24 (pseudo-t, ${ }^{3} \mathrm{~J}_{\mathrm{H}-\mathrm{H}}=2.0 \mathrm{~Hz}$, $2 \mathrm{H}, H 9$ ), 4.23 (pseudo-t, ${ }^{3} \mathrm{~J}_{\mathrm{H}-\mathrm{H}}=2.0 \mathrm{~Hz}, 2 \mathrm{H}, \mathrm{H15}$ ), 4.20 (pseudo-t, ${ }^{3} \mathrm{~J}_{\mathrm{H}-\mathrm{H}}=2.0 \mathrm{~Hz}, 2 \mathrm{H}, H 13$ ), 4.14 (pseudo$\mathrm{t},{ }^{3} \mathrm{~J}_{\mathrm{H}-\mathrm{H}}=2.0 \mathrm{~Hz}, 2 \mathrm{H}, \mathrm{H7}$ ), 4.07 (pseudo-t, ${ }^{3} \mathrm{~J}_{\mathrm{H}-\mathrm{H}}=2.0 \mathrm{~Hz}, 2 \mathrm{H}, \mathrm{H16}$ ), $2.50(\mathrm{~s}, 3 \mathrm{H}, \mathrm{H} 24) \mathrm{ppm} ;{ }^{13} \mathrm{C}\left\{{ }^{1} \mathrm{H}\right\} \mathrm{NMR}$ $\left(\mathrm{CDCl}_{3}, 298 \mathrm{~K}, 100 \mathrm{MHz}\right): \delta_{\mathrm{c}}=149.6(C 2), 138.4(C 23), 132.4$ (C3), $131.8(C 21), 126.0$ (C22), 125.7 (C1), 120.6 (C20), 94.2 (C5), 88.3 (C18), 85.9 (C19), 84.9 (C4), 83.9 (C11/12), 83.9 (C11/12), 73.1 (C8), 72.8 (C9), 70.7 (C7), 70.1 (C16), 69.9 (C13/C15), 69.8 (C13/C15), 68.4 (C10/C14), 68.3 (C10/C14), 66.3 (C17), 64.6 (C6), 15.7 (C24) ppm; IR: 2207 (-C $\equiv C$-) $\mathrm{cm}^{-1}$; MS ES+: calcd. for $\mathrm{C}_{38} \mathrm{H}_{28} \mathrm{Fe}_{2} \mathrm{NS}$ [M]+ 618.0641; found. 618.0650 .

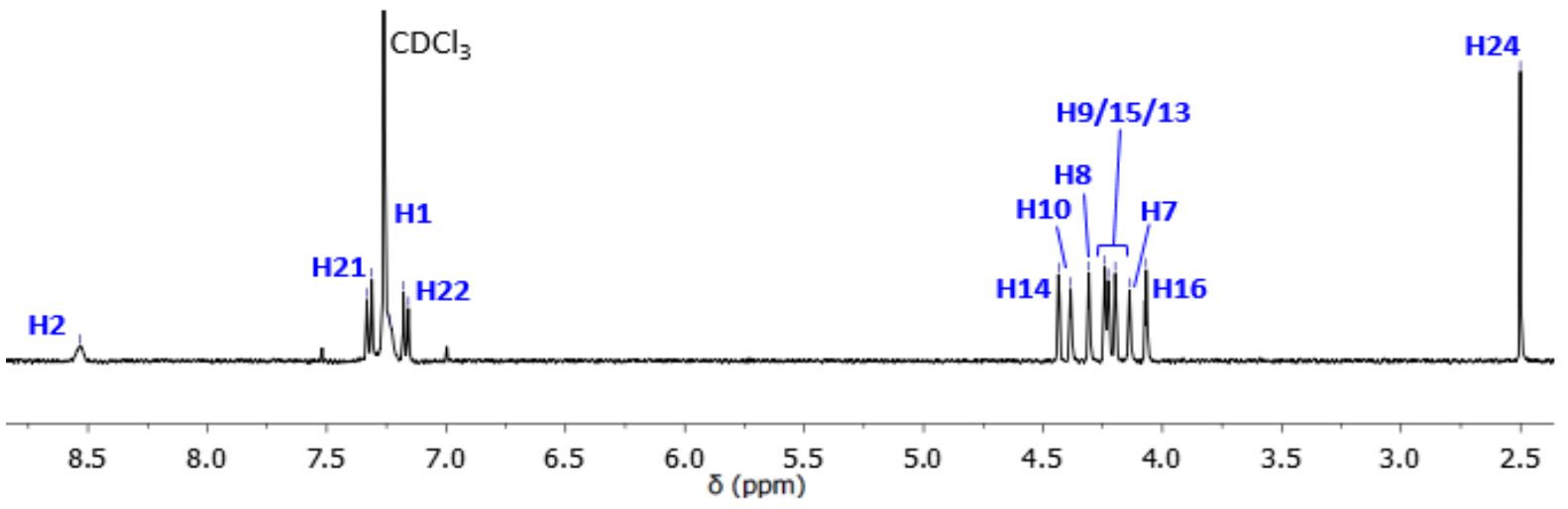

Figure S39: The ${ }^{1} \mathrm{H}$ NMR spectrum of $7 \mathrm{ac}$ in $\mathrm{CDCl}_{3}$

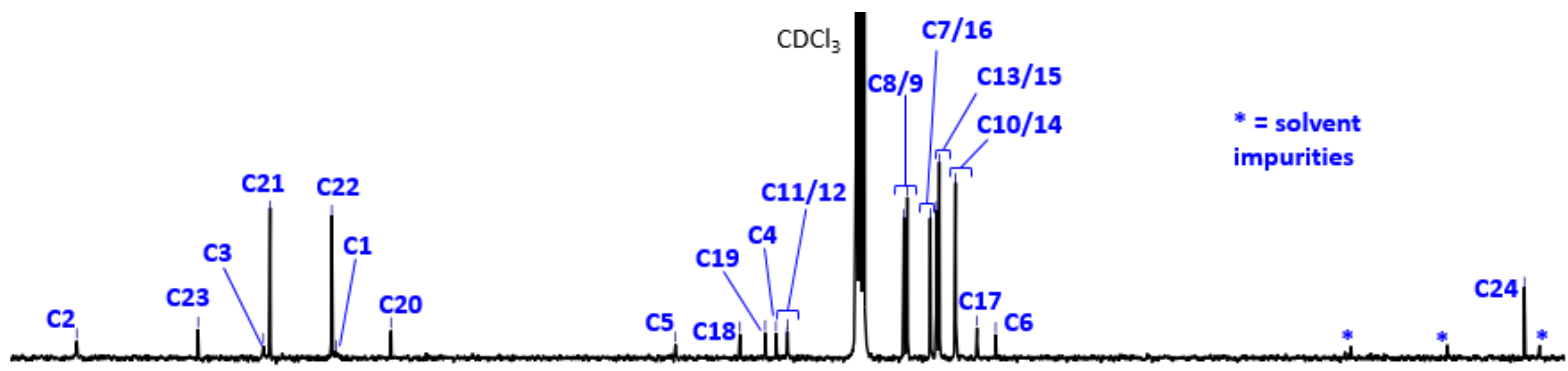

\begin{tabular}{|c|c|c|c|c|c|c|c|c|c|c|c|c|c|}
\hline 150 & 140 & 130 & 120 & 110 & 100 & 90 & 80 & 70 & 60 & 50 & 40 & 30 & 20 \\
\hline
\end{tabular}

Figure S40: The ${ }^{13} \mathrm{C}\left\{{ }^{1} \mathrm{H}\right\}$ NMR spectrum of $7 \mathrm{ac}$ in $\mathrm{CDCl}_{3}$ 
1,1'"-Di(4-(ethyny)thioanisole)biferrocene: (7cc) $)^{2}$

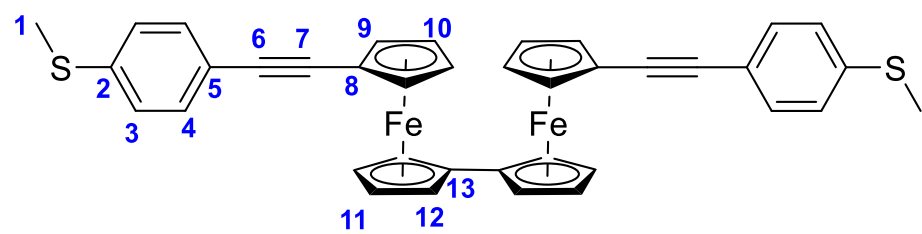

Following the general procedure, 1,1'"-diiodobiferrocene $(0.10 \mathrm{~g}, 0.16 \mathrm{mmol}), \mathrm{Cul}(0.01 \mathrm{~g}, 0.01 \mathrm{mmol})$, 4-ethynylthioanisole $(0.05 \mathrm{~g}, 0.32 \mathrm{mmol})$ and $\mathrm{Pd}\left(\mathrm{P}^{\mathrm{t}} \mathrm{Bu}_{3}\right)_{2}(0.01 \mathrm{~g}, 0.01 \mathrm{mmol})$ gave a brown solid which was purified by chromatography on a silica column, eluted with $n$-hexane/DCM (1:0 $\rightarrow$ 1:1 v/v) to give the product as an orange solid (0.04 g, $0.06 \mathrm{mmol}, 37 \%)$.

${ }^{1} \mathrm{H}$ NMR $\left(\mathrm{CDCl}_{3}, 298 \mathrm{~K}, 400 \mathrm{MHz}\right): \delta_{\mathrm{H}}=7.31\left(\mathrm{~d},{ }^{3} \int_{\mathrm{H}-\mathrm{H}}=4.4 \mathrm{~Hz}, 4 \mathrm{H}, \mathrm{H4}\right), 7.17\left(\mathrm{~d},{ }^{3} \mathrm{~J}_{\mathrm{H}-\mathrm{H}}=4.4 \mathrm{~Hz}, 4 \mathrm{H}, \mathrm{H3}\right)$, 4.41 (pseudo-t, ${ }^{3} \mathrm{~J}_{\mathrm{H}-\mathrm{H}}=2.0 \mathrm{~Hz}, 4 \mathrm{H}, \mathrm{H11}$ ), 4.25 (pseudo-t, ${ }^{3} \mathrm{~J}_{\mathrm{H}-\mathrm{H}}=2.0 \mathrm{~Hz}, 4 \mathrm{H}, \mathrm{H10}$ ), 4.22 (pseudo-t, ${ }^{3} \mathrm{~J}_{\mathrm{H}-\mathrm{H}}=$ $2.0 \mathrm{~Hz}, 4 \mathrm{H}, \mathrm{H12}$ ), 4.07 (pseudo-t, ${ }^{3} \mathrm{H}_{\mathrm{H}-\mathrm{H}}=2.0 \mathrm{~Hz}, 4 \mathrm{H}, \mathrm{H9}$ ), $2.50(\mathrm{~s}, 6 \mathrm{H}, \mathrm{H1}) \mathrm{ppm} ;{ }^{13} \mathrm{C}\left\{{ }^{1} \mathrm{H}\right\} \mathrm{NMR}\left(\mathrm{CDCl}_{3}, 298\right.$ $\mathrm{K}, 100 \mathrm{MHz}$ ): $\delta_{\mathrm{c}}=138.3$ (C2), 131.8 (C4), 126.1 (C3), 120.6 (C5), 88.4 (C7), 85.9 (C6), 84.4 (C13), 72.8 (C10), 70.1 (C9), 69.8 (C12), 68.5 (C11), 66.2 (C8), 15.7 (C1) ppm; IR: 2209 (-C=C-) cm-1; MS ES+: calcd. for $\mathrm{C}_{38} \mathrm{H}_{31} \mathrm{Fe}_{2} \mathrm{~S}_{2}[\mathrm{M}]+663.0561$; found. 663.0548 .

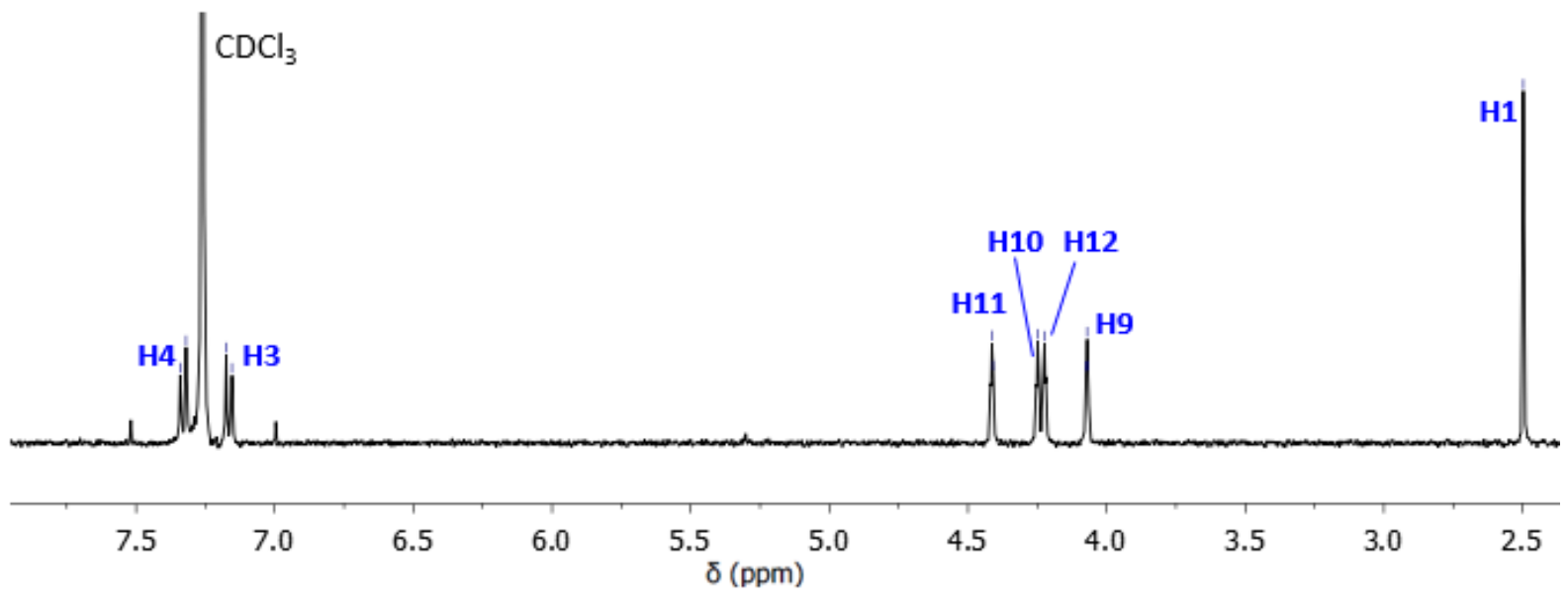

Figure S41: The ${ }^{1} \mathrm{H}$ NMR spectrum of $7 \mathrm{cc}$ in $\mathrm{CDCl}_{3}$

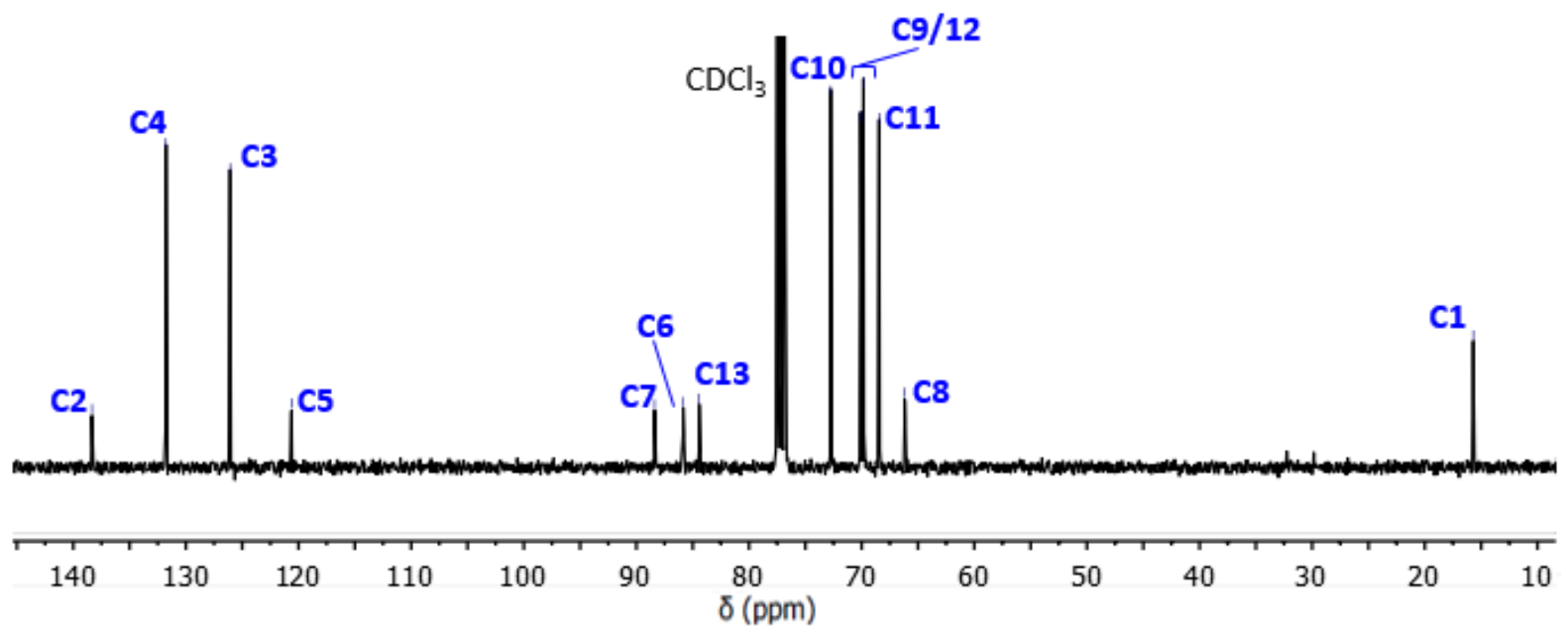

Figure S42: The ${ }^{13} \mathrm{C}\left\{{ }^{1} \mathrm{H}\right\}$ NMR spectrum of $7 \mathrm{cc}$ in $\mathrm{CDCl}_{3}$ 


\section{Cyclic Voltammetry}

\subsection{Experimental}

Cyclic voltammograms of compounds in solution were recorded under an atmosphere of nitrogen in $\mathrm{CH}_{2} \mathrm{Cl}_{2} / 0.1 \mathrm{M}\left[\mathrm{nBu}_{4}\right]\left[\mathrm{PF}_{6}\right]$ on a Gamry reference 600TM (Gamry Instruments, Warminter, PA, USA) potentiostat with a glassy carbon disc as the working electrode (diameter $=2.5 \mathrm{~mm}$ ), and Pt-wire as reference and counter electrodes. Analyte solutions were between 0.1-1 mM. Potentials are reported relative to $\left[\mathrm{Cp}_{2} \mathrm{Fe}\right]^{+} /\left[\mathrm{Cp}_{2} \mathrm{Fe}\right]$, measured against internal $\left[\mathrm{Cp} *{ }_{2} \mathrm{Fe}\right]+/\left[\mathrm{Cp} *{ }_{2} \mathrm{Fe}\right]$ references.
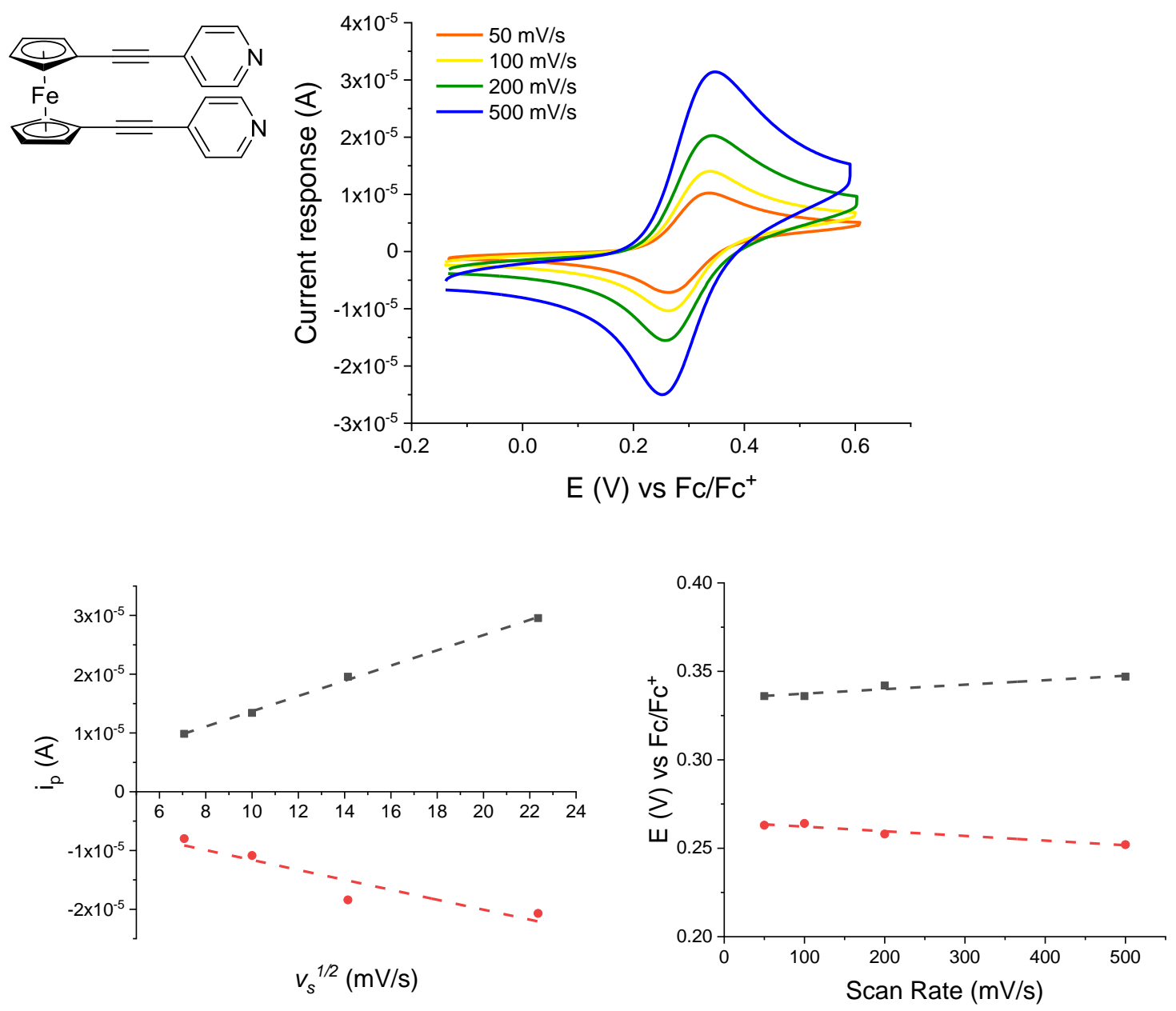

Figure S43: Scan rate dependent cyclic voltammetry of 3aa (top), with linear fits to compare the scan rates to their respective peak currents (bottom left) and the peak potentials (bottom right), structure inset. 

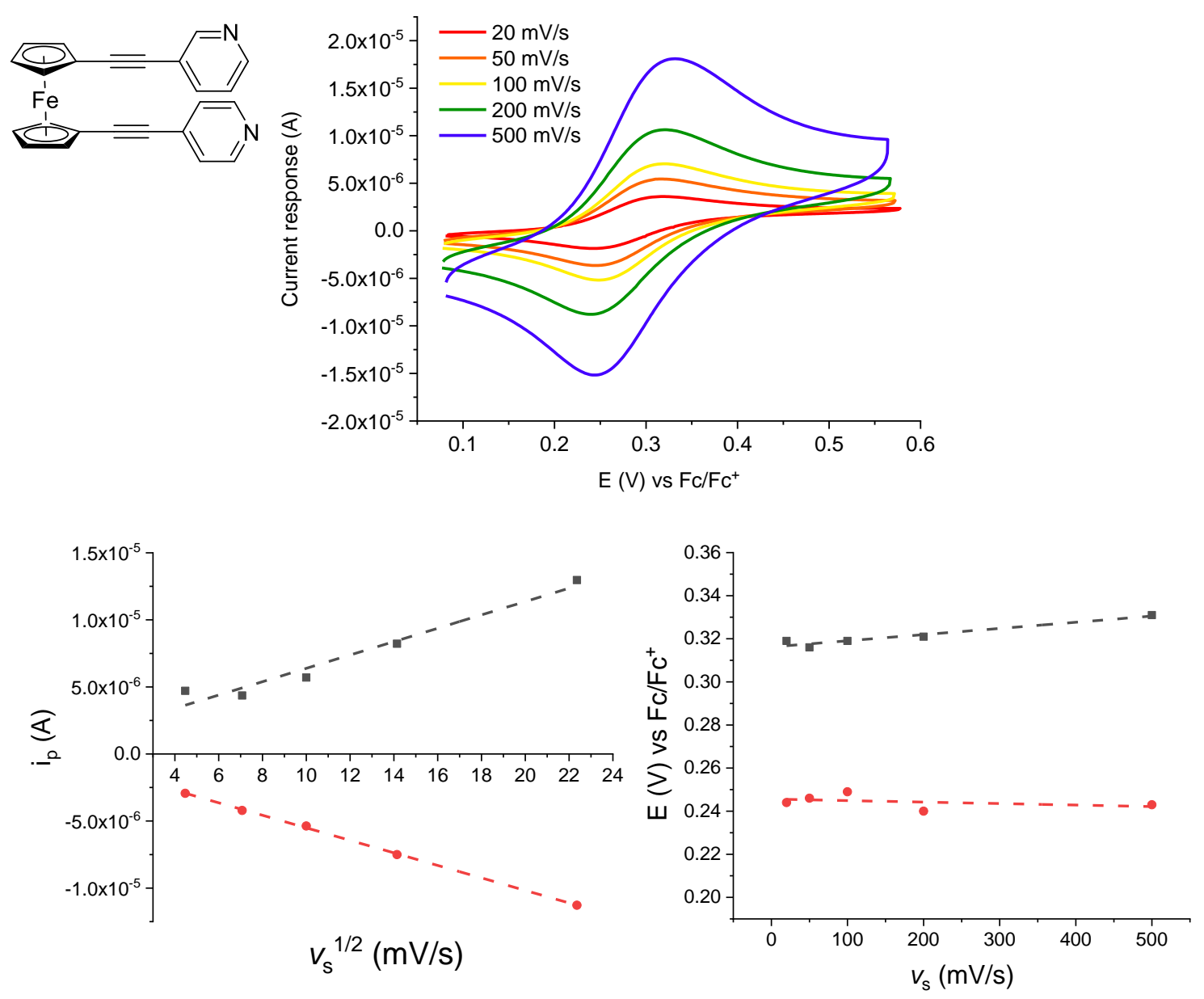

Figure S44: Scan rate dependent cyclic voltammetry of $\mathbf{3 a b}$ (top), with linear fits to compare the scan rates to their respective peak currents (bottom left) and the peak potentials (bottom right), structure inset. 

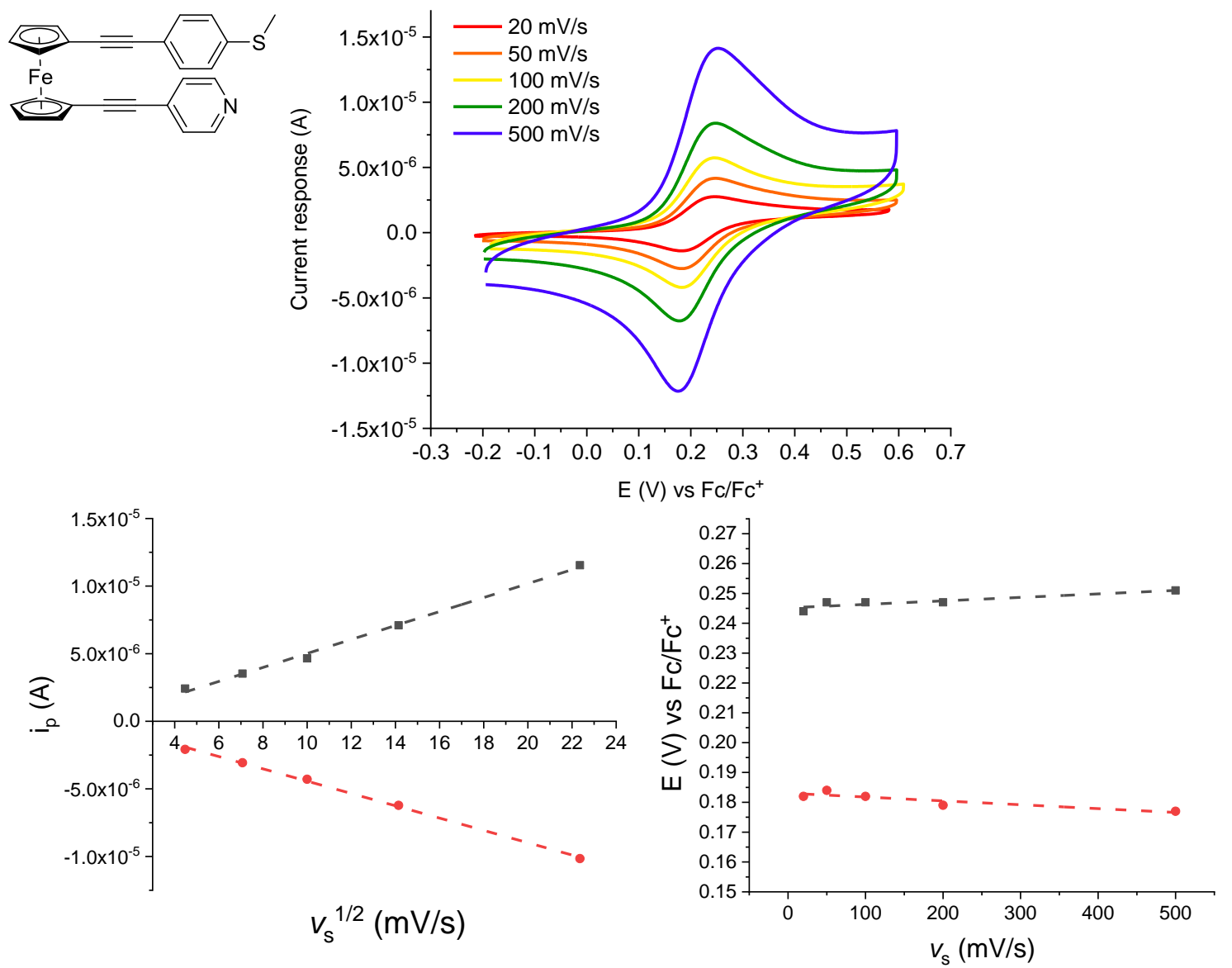

Figure S45: Scan rate dependent cyclic voltammetry of 3ac (top), with linear fits to compare the scan rates to their respective peak currents (bottom left) and the peak potentials (bottom right), structure inset. 

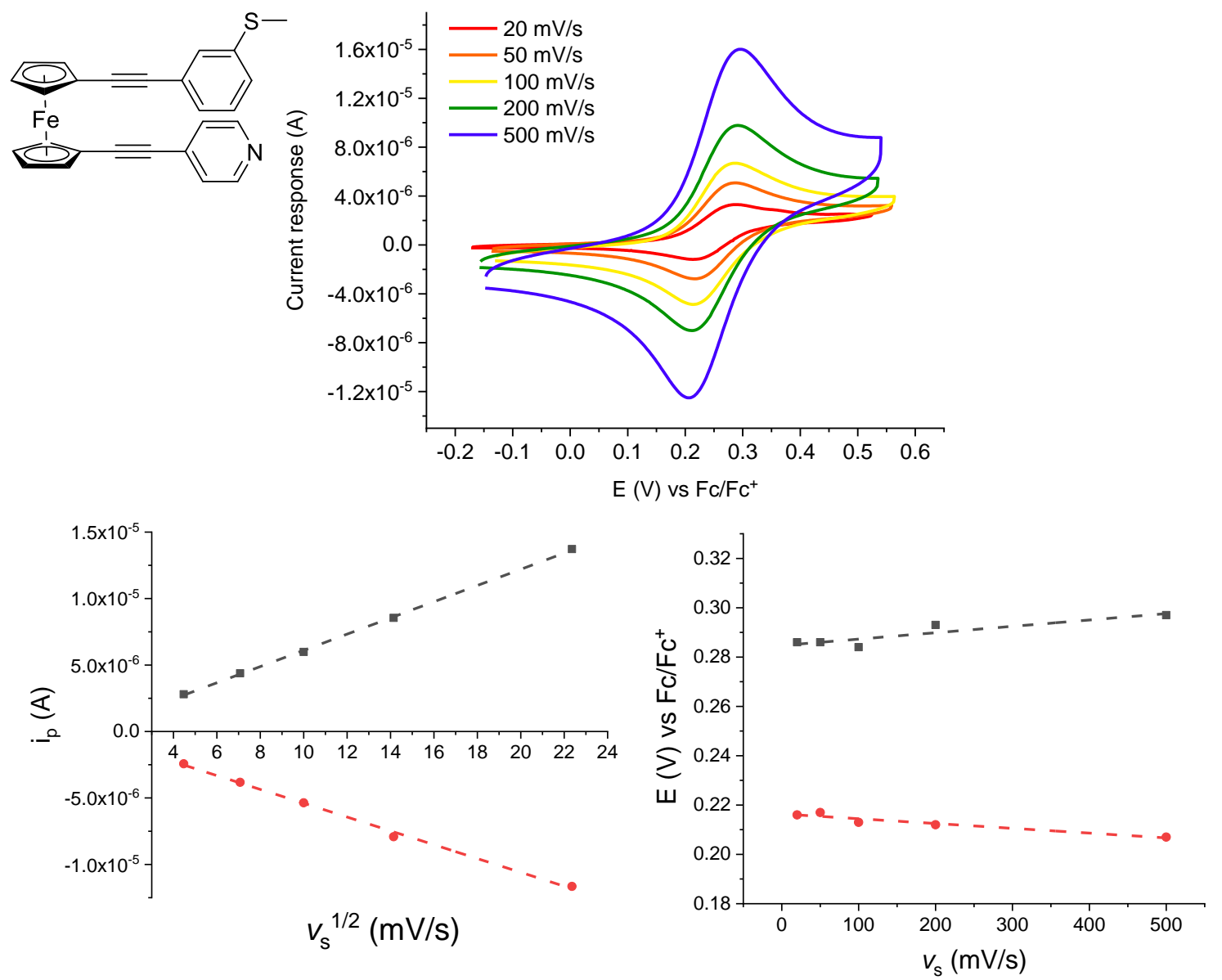

Figure S46: Scan rate dependent cyclic voltammetry of 3ad (top), with linear fits to compare the scan rates to their respective peak currents (bottom left) and the peak potentials (bottom right), structure inset. 

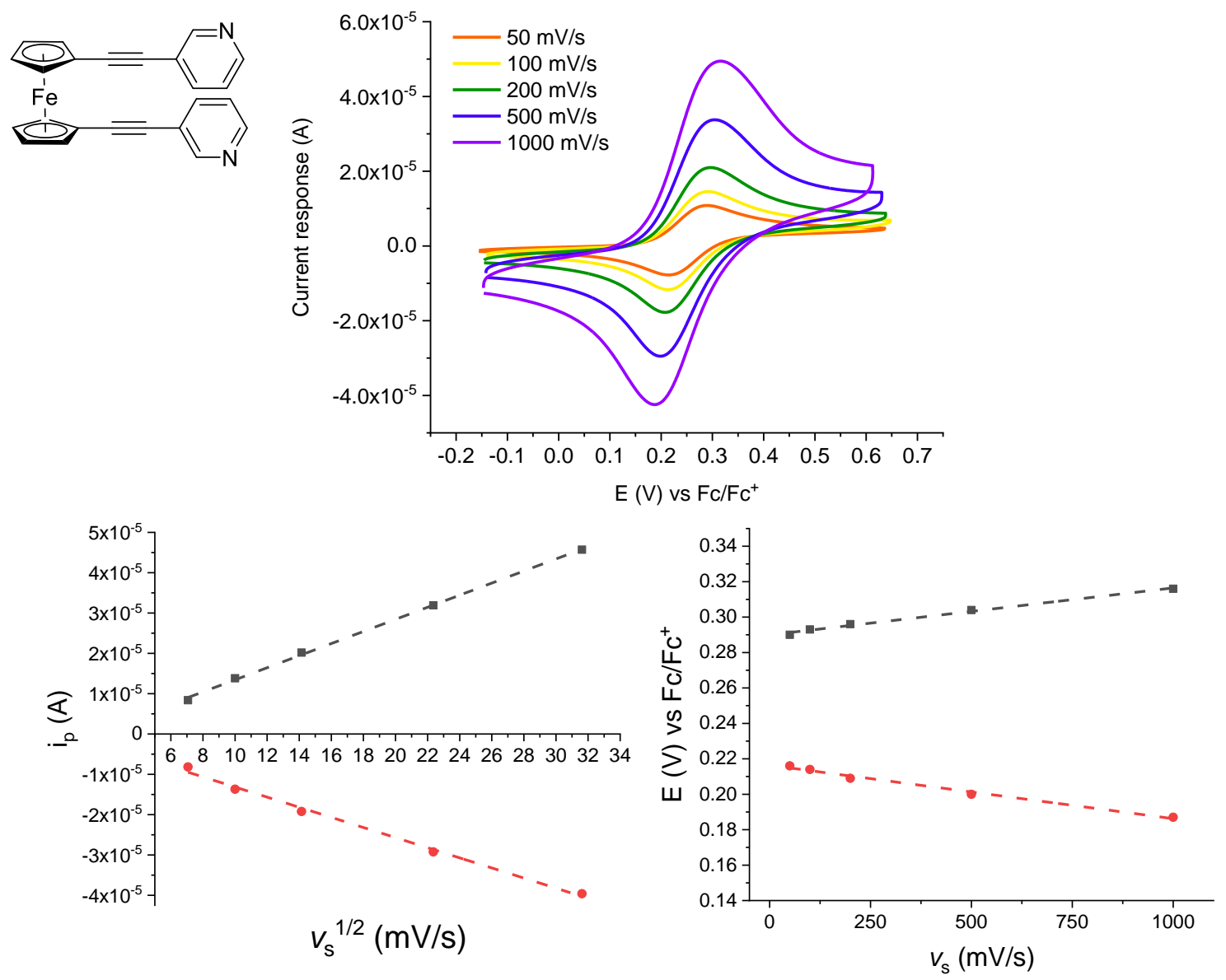

Figure S47: Scan rate dependent cyclic voltammetry of $\mathbf{3 b b}$ (top), with linear fits to compare the scan rates to their respective peak currents (bottom left) and the peak potentials (bottom right), structure inset. 

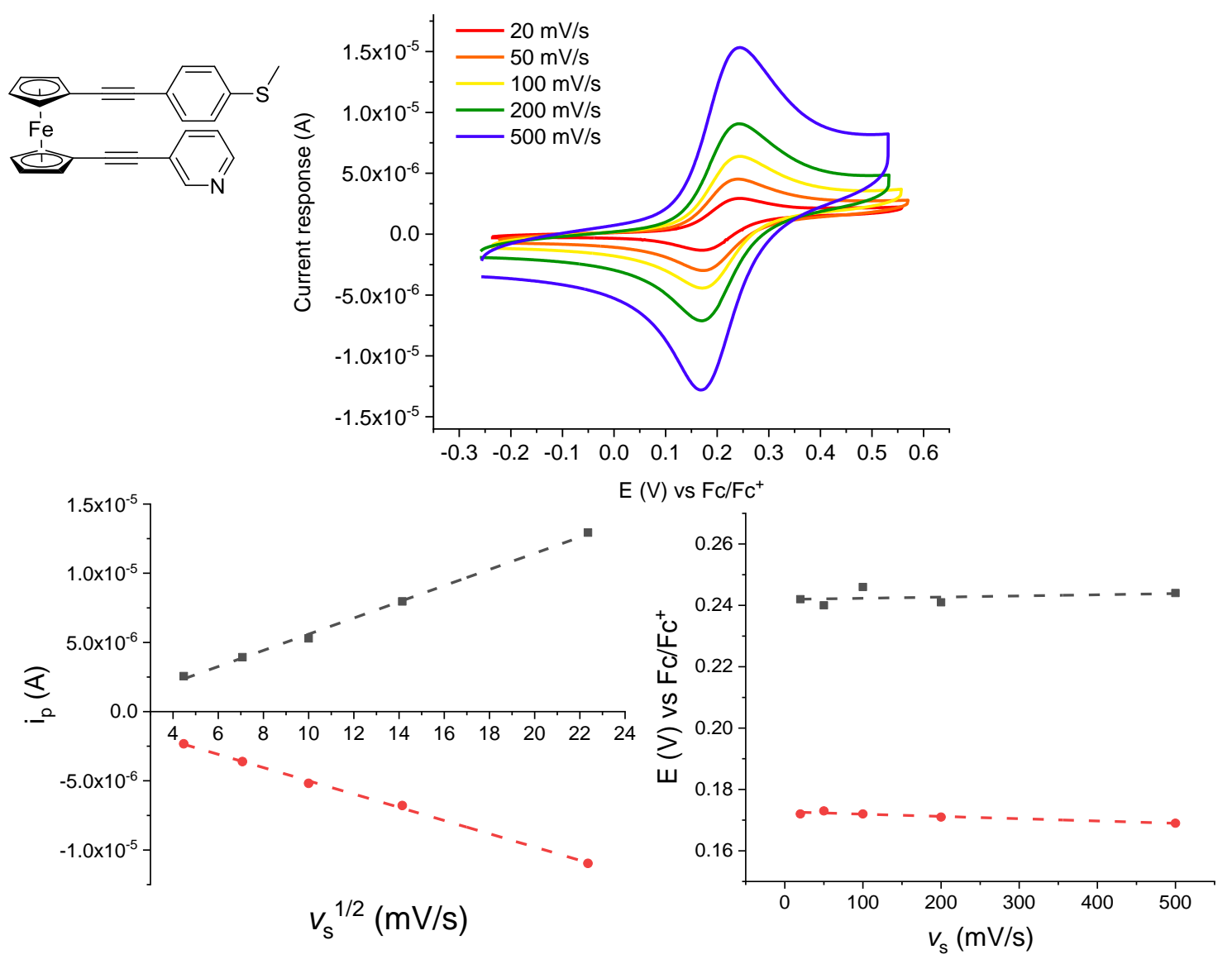

Figure S48: Scan rate dependent cyclic voltammetry of $\mathbf{3 b c}$ (top), with linear fits to compare the scan rates to their respective peak currents (bottom left) and the peak potentials (bottom right), structure inset. 

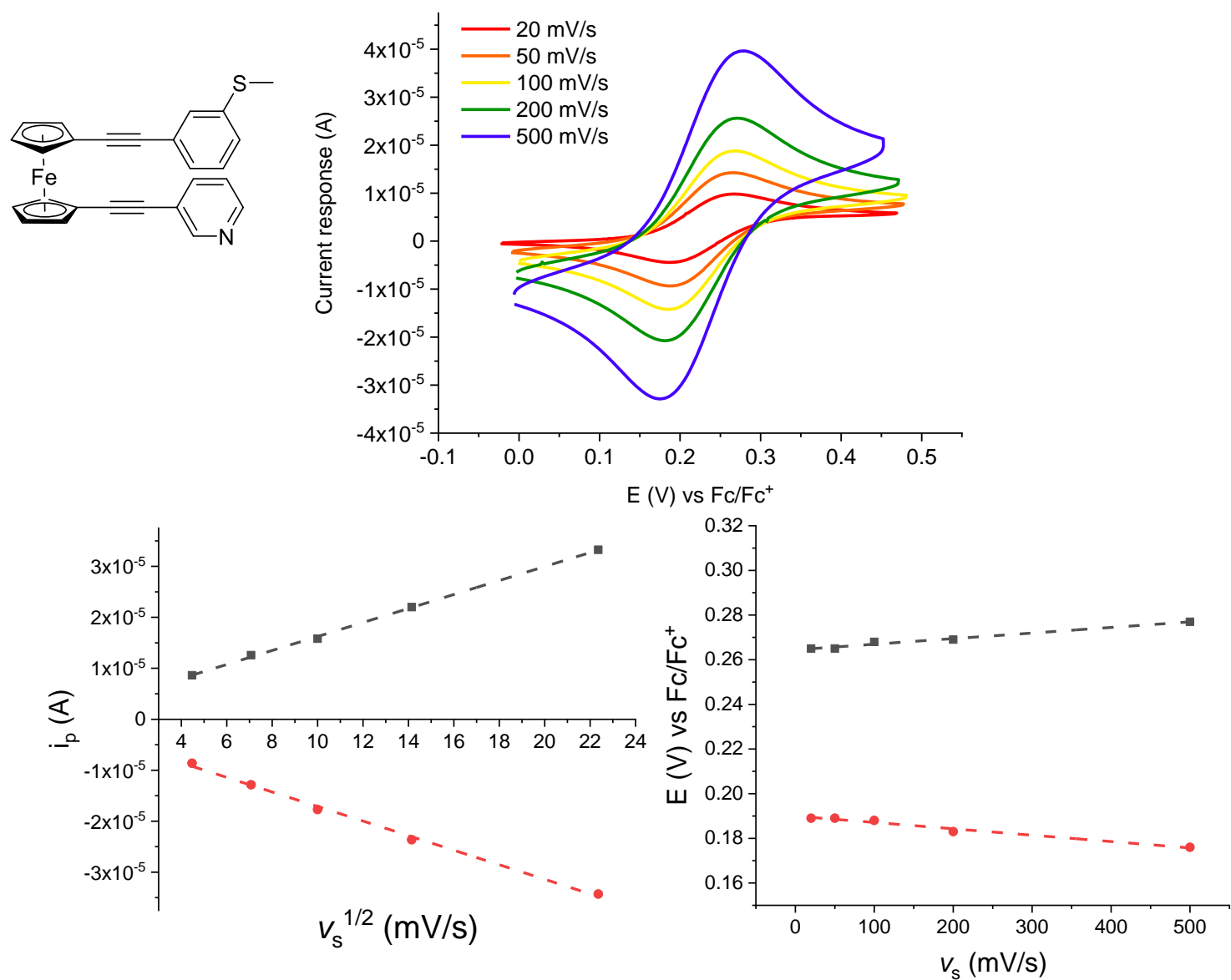

Figure S49: Scan rate dependent cyclic voltammetry of 3bd (top), with linear fits to compare the scan rates to their respective peak currents (bottom left) and the peak potentials (bottom right), structure inset. 

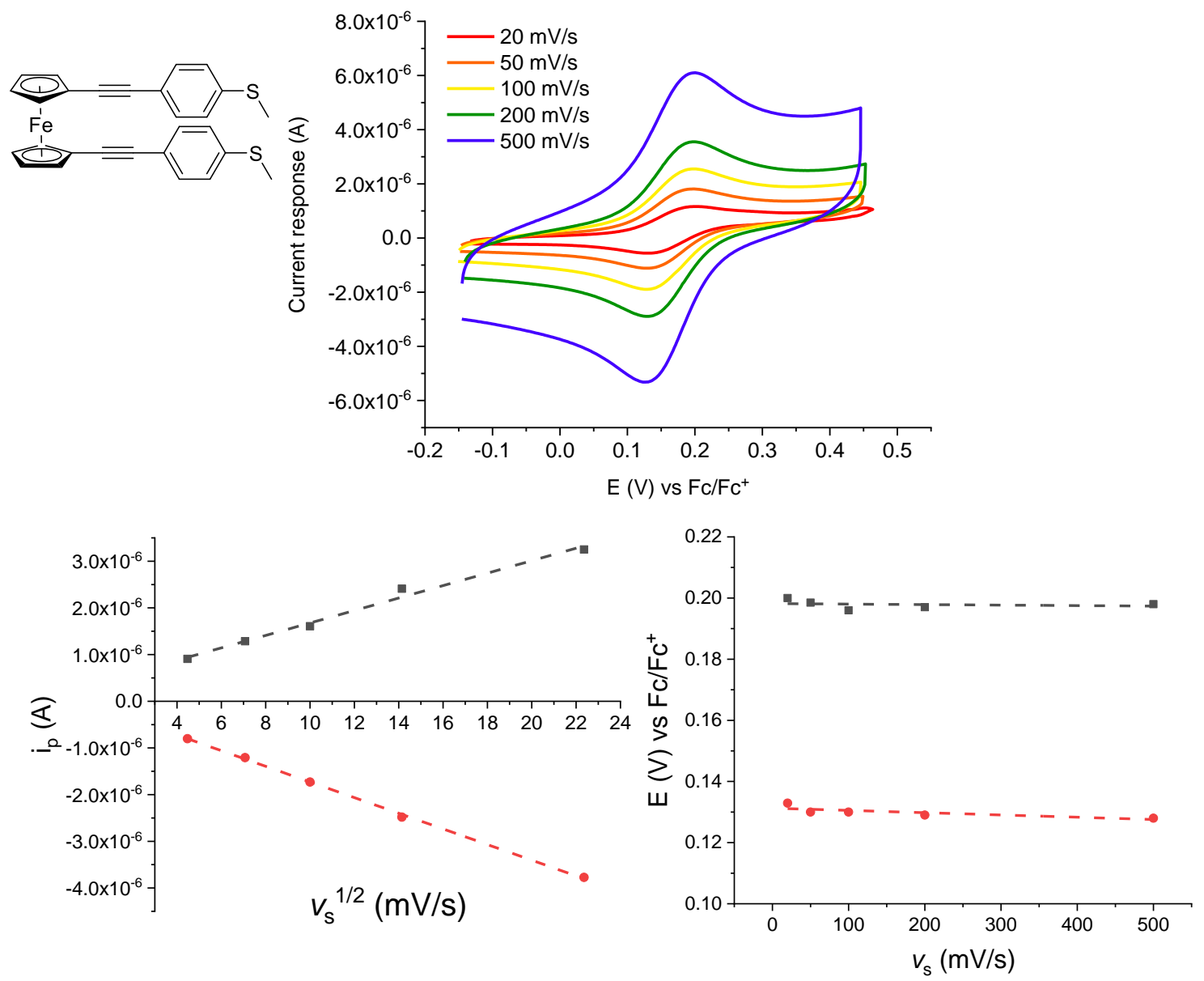

Figure S50: Scan rate dependent cyclic voltammetry of 3cc (top), with linear fits to compare the scan rates to their respective peak currents (bottom left) and the peak potentials (bottom right), structure inset. 

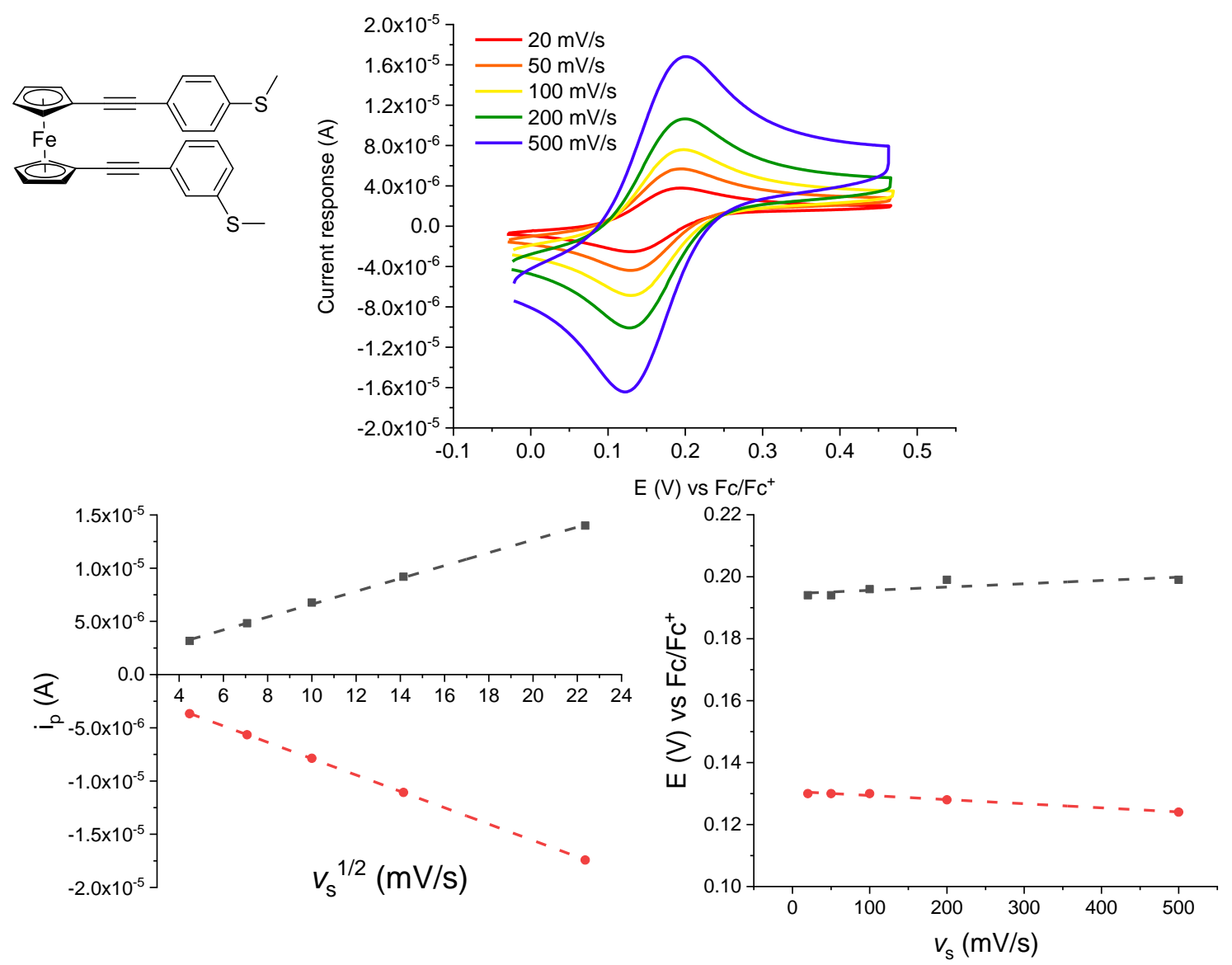

Figure S51: Scan rate dependent cyclic voltammetry of $\mathbf{3 c d}$ (top), with linear fits to compare the scan rates to their respective peak currents (bottom left) and the peak potentials (bottom right), structure inset. 

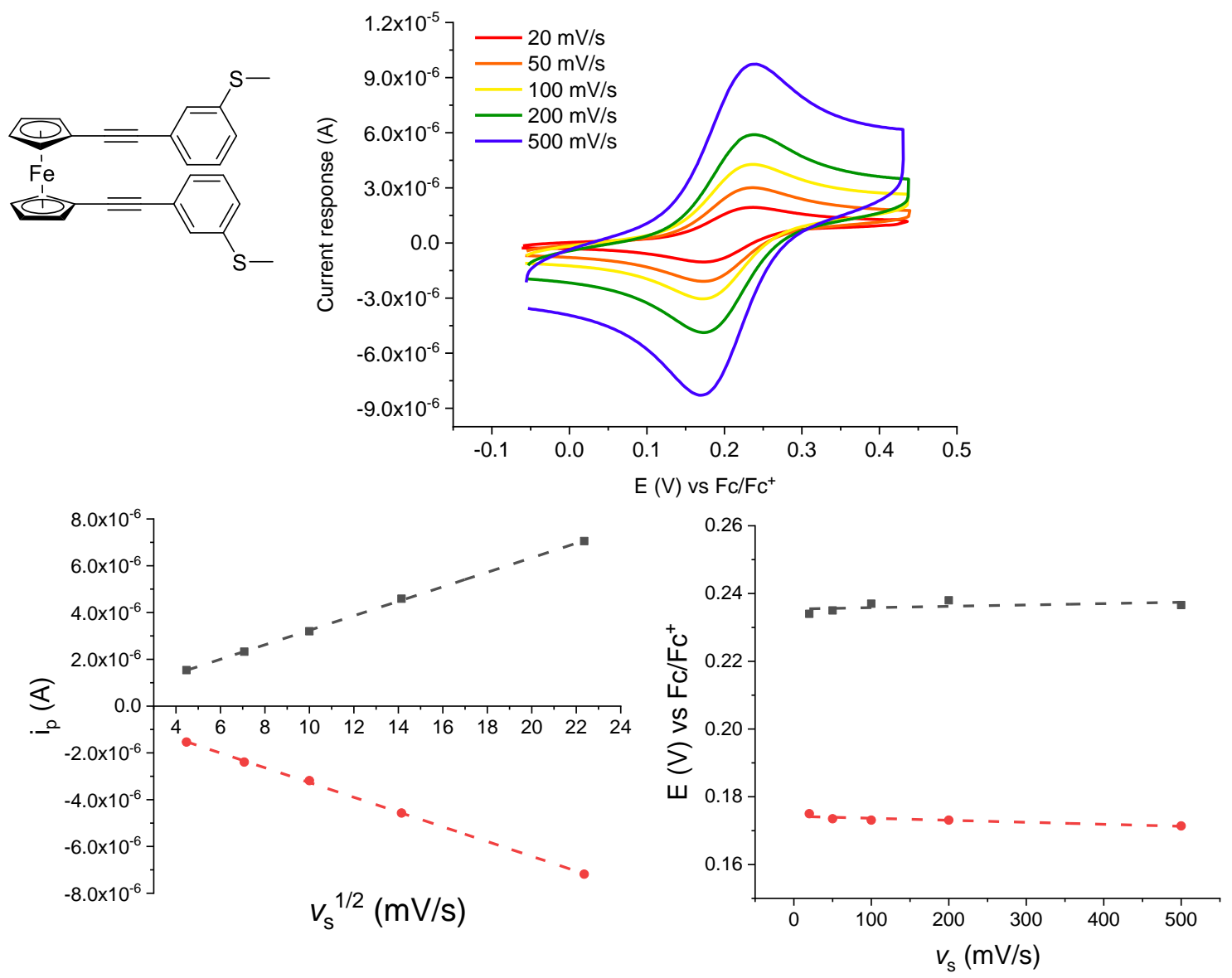

Figure S52: Scan rate dependent cyclic voltammetry of 3dd (top), with linear fits to compare the scan rates to their respective peak currents (bottom left) and the peak potentials (bottom right), structure inset. 

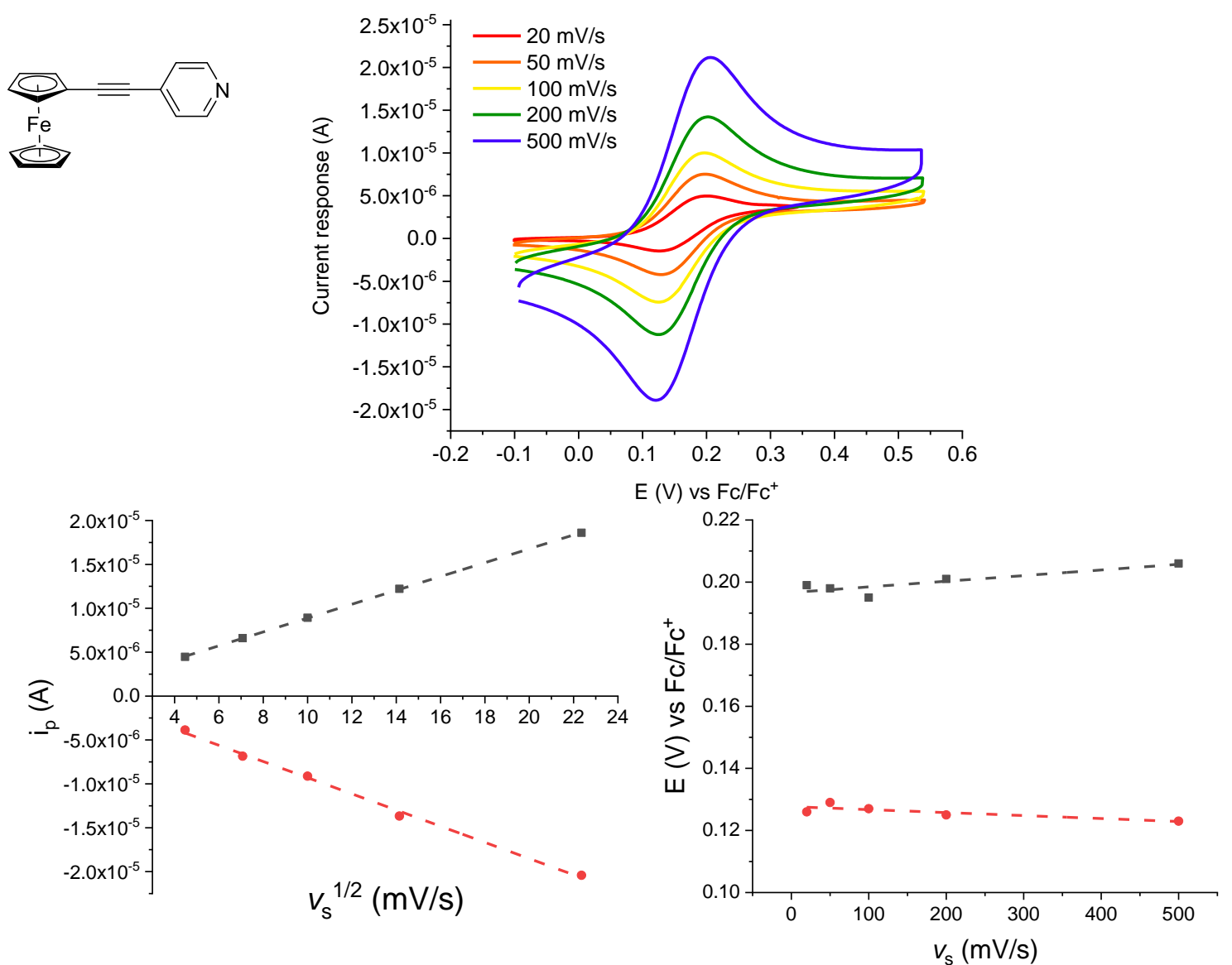

Figure S53: Scan rate dependent cyclic voltammetry of 4a (top), with linear fits to compare the scan rates to their respective peak currents (bottom left) and the peak potentials (bottom right), structure inset. 

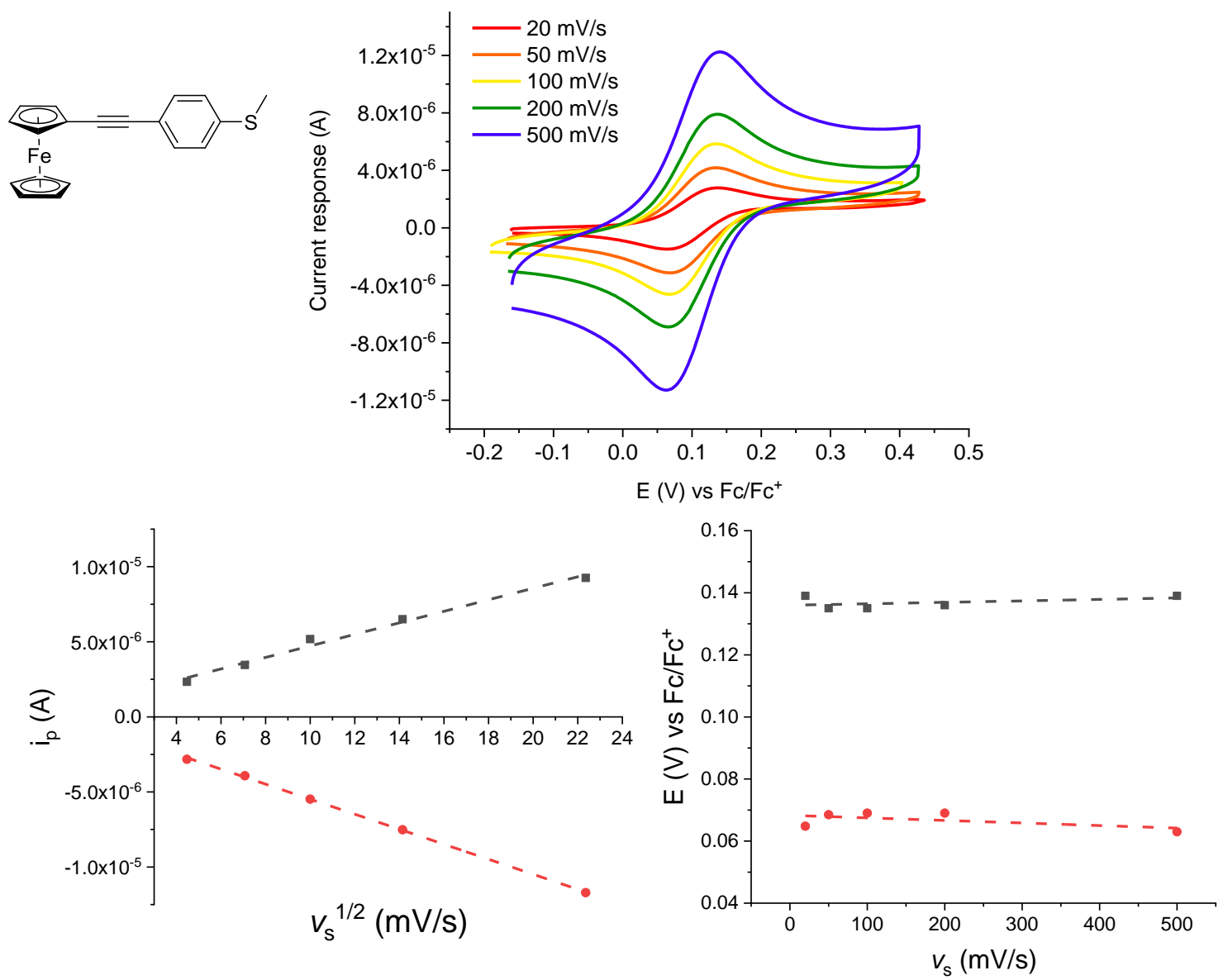

Figure S54: Scan rate dependent cyclic voltammetry of $\mathbf{4 c}$ (top), with linear fits to compare the scan rates to their respective peak currents (bottom left) and the peak potentials (bottom right), structure inset. 

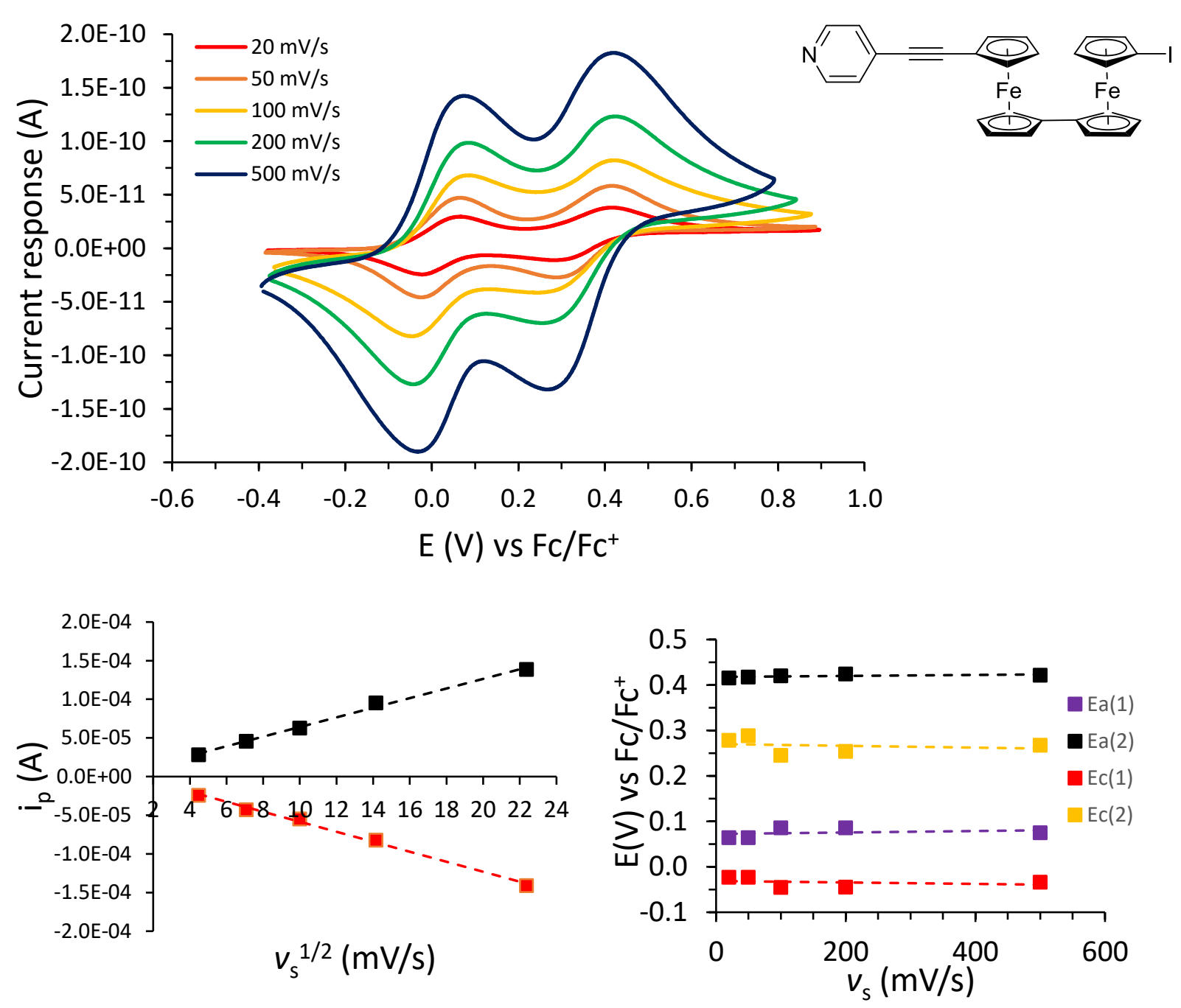

Figure S55: Scan rate dependent cyclic voltammetry of 6 a (top), with linear fits to compare the scan rates to their respective peak currents (bottom left) and the peak potentials (bottom right), structure inset. 

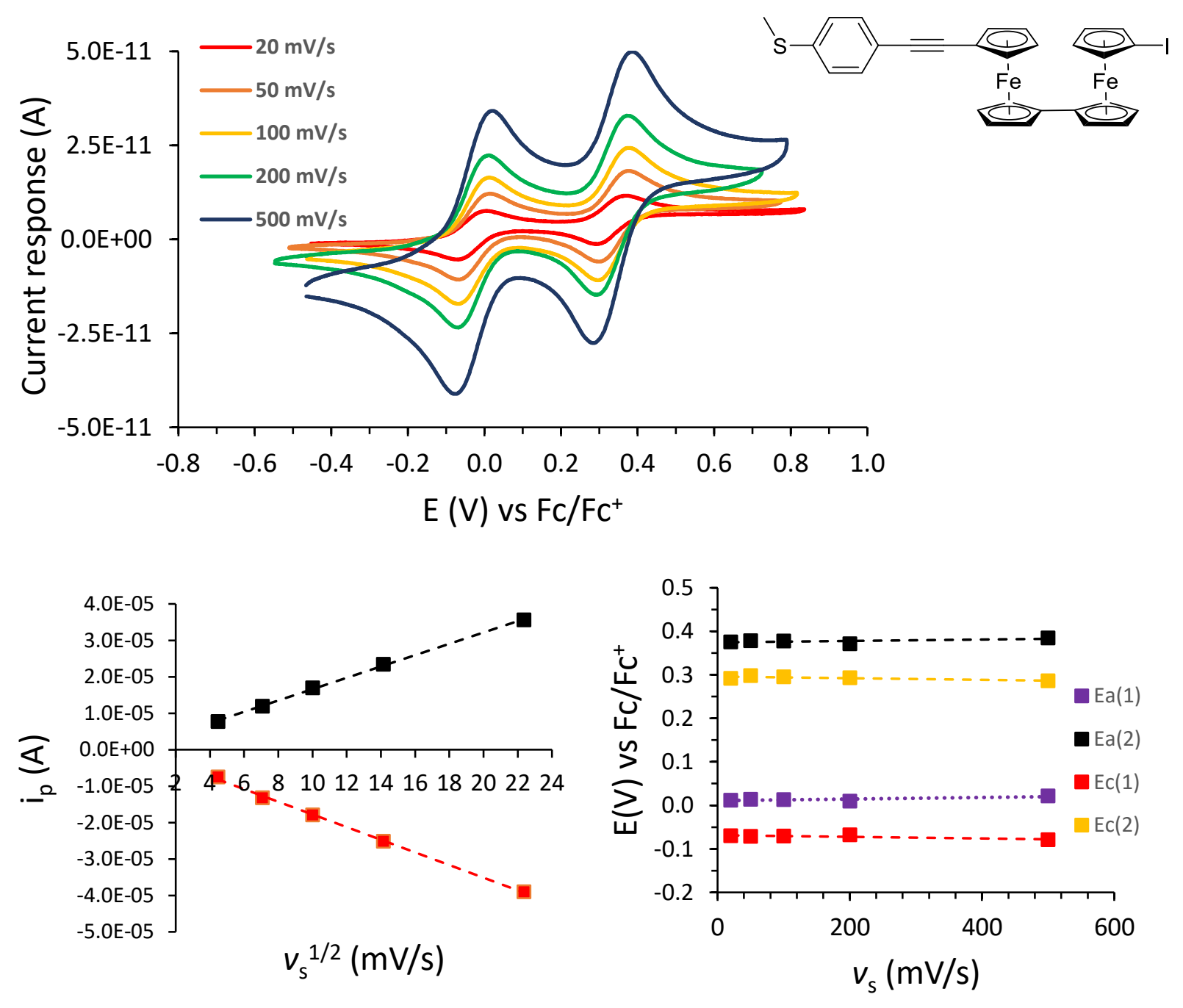

Figure S56: Scan rate dependent cyclic voltammetry of $\mathbf{6 c}$ (top), with linear fits to compare the scan rates to their respective peak currents (bottom left) and the peak potentials (bottom right), structure inset. 


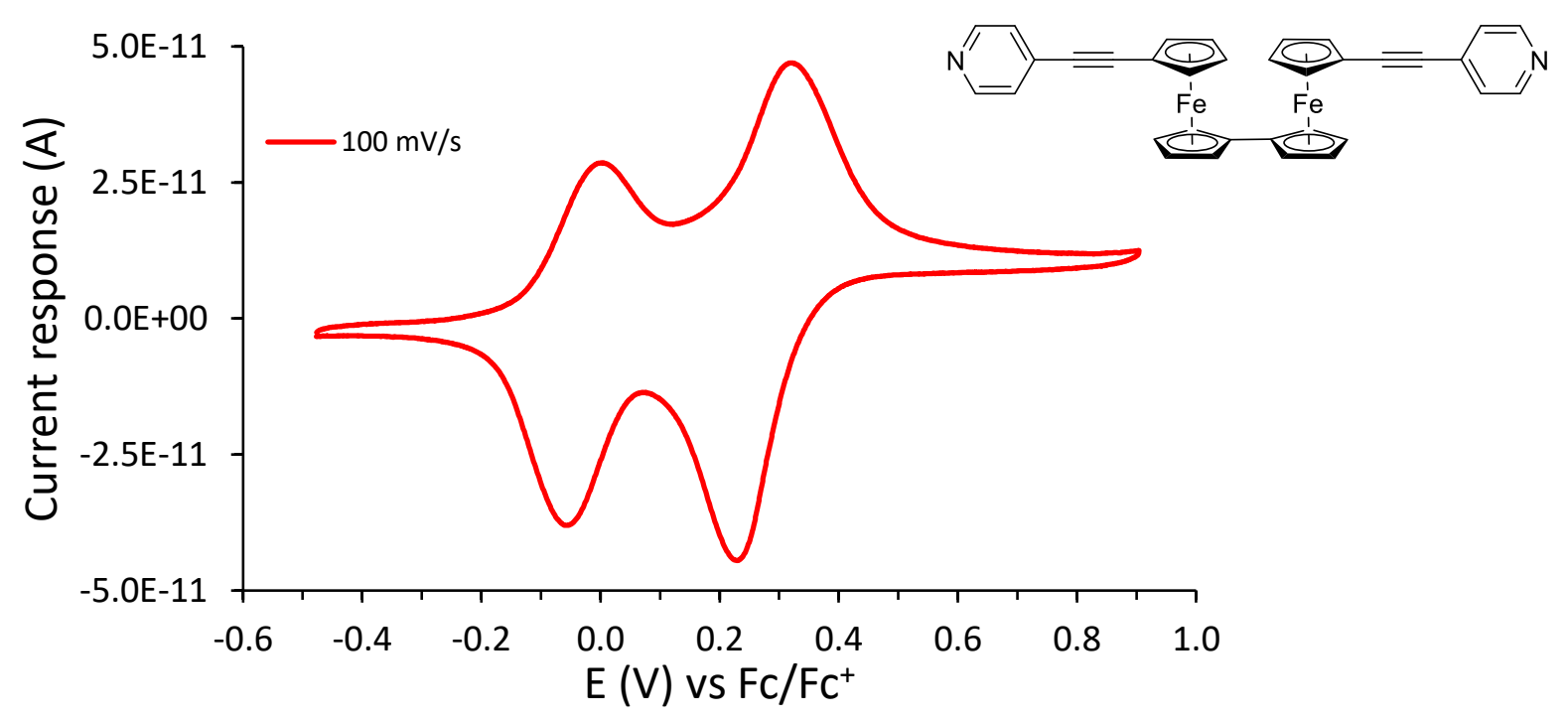

Figure S57: Cyclic voltammetry collected at $100 \mathrm{mV} / \mathrm{s}$ for compound 7aa. A full scan rate dependency was not obtained for this system as it showed significant instability under the conditions we used.

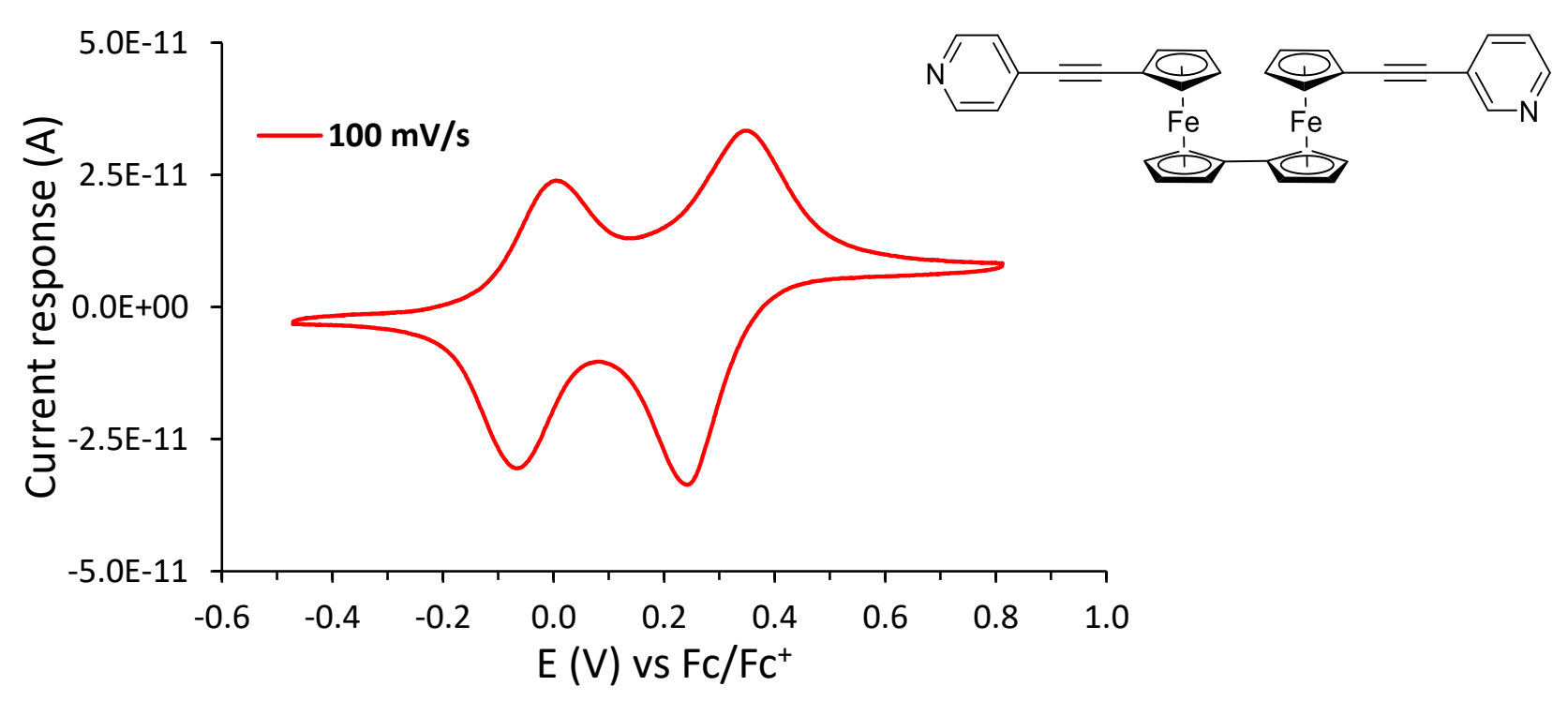

Figure S58: Cyclic voltammetry collected at $100 \mathrm{mV} / \mathrm{s}$ for compound 7ab. A full scan rate dependency was not obtained for this system as it showed significant instability under the conditions we used. 

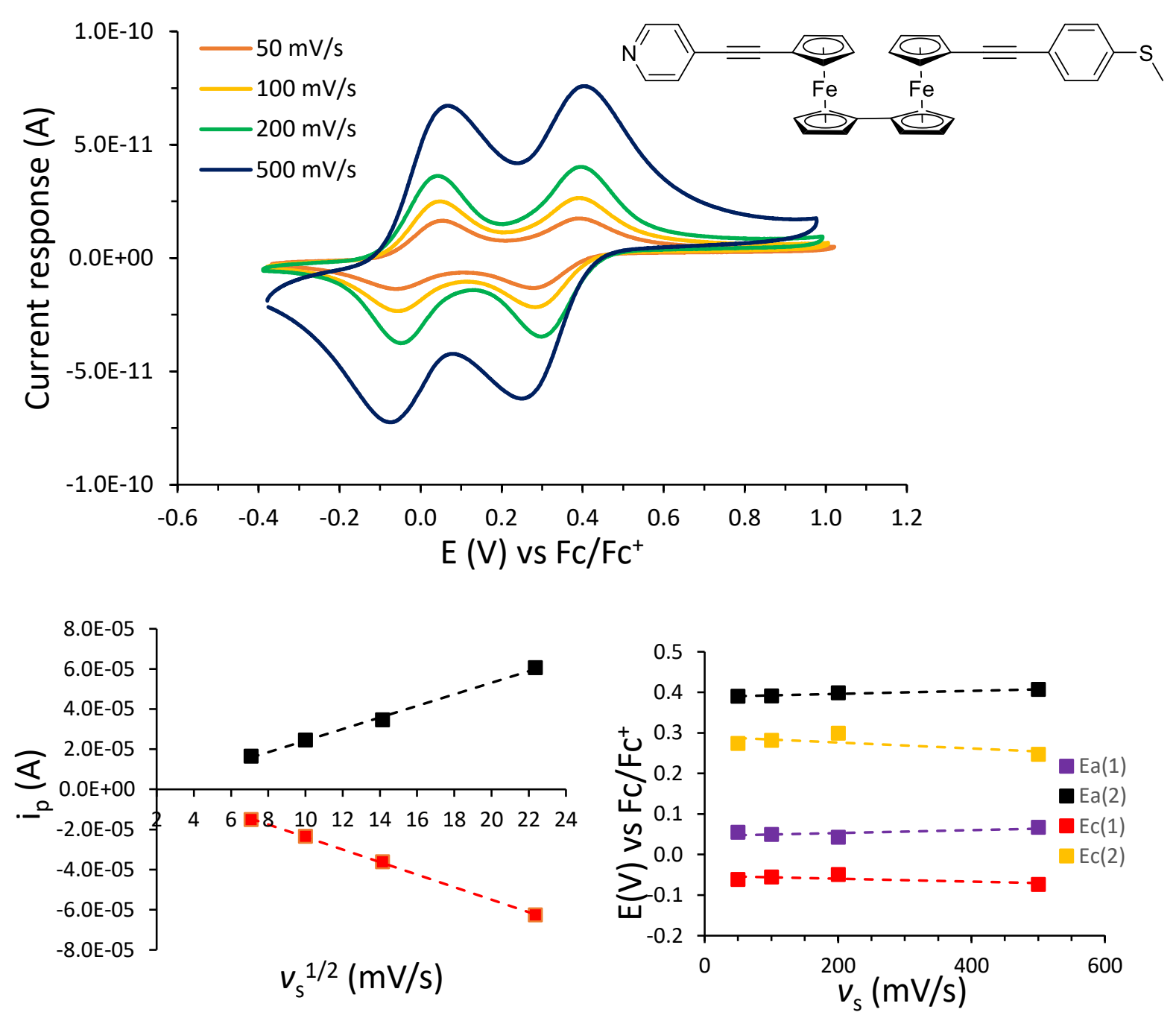

Figure S59: Scan rate dependent cyclic voltammetry of 7ac (top), with linear fits to compare the scan rates to their respective peak currents (bottom left) and the peak potentials (bottom right), structure inset. 

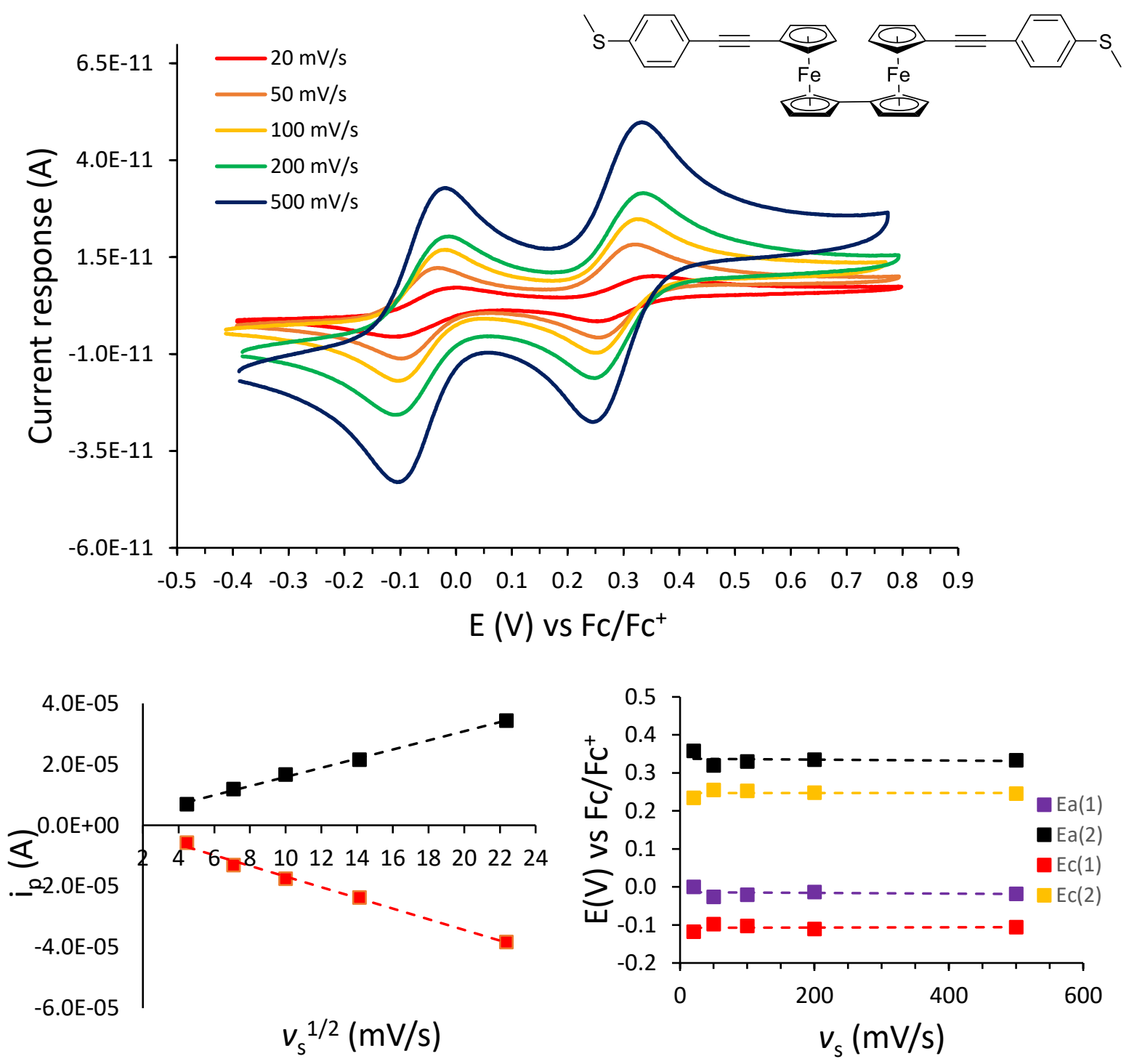

Figure S60: Scan rate dependent cyclic voltammetry of 7cc (top), with linear fits to compare the scan rates to their respective peak currents (bottom left) and the peak potentials (bottom right), structure inset. 


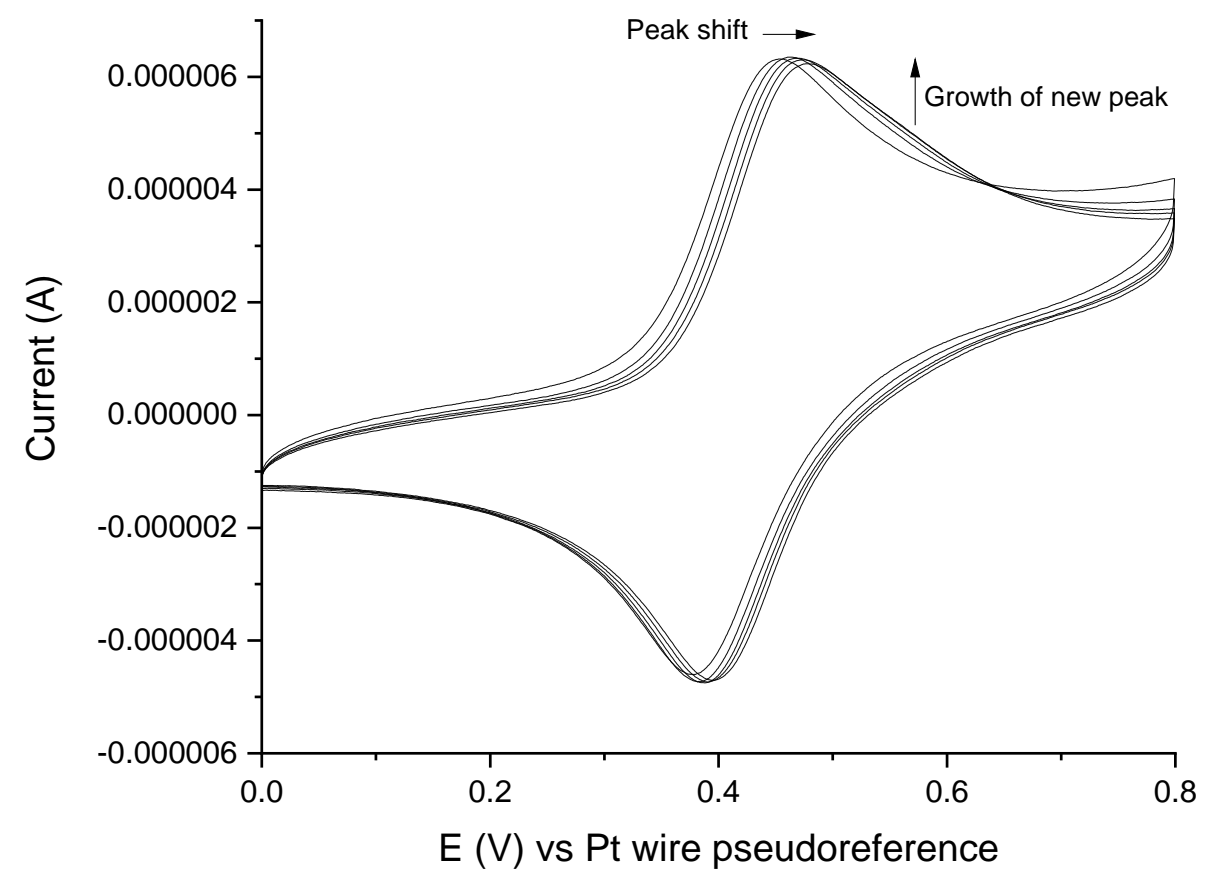

Figure S61: The CV of compound 3ac over 17 cycles (some traces omitted for clarity) showing a clear change in the redox profile of the molecule with sequential scans. 


\section{UV/Vis Spectroscopy}

Table S1: Peak positions obtained from UV/Vis spectroscopy of compounds $\mathbf{3 x x}, \mathbf{4 x}, \mathbf{6 x}$ and $\mathbf{7 x x}$. All data were recorded at room temperature in $\mathrm{CH}_{2} \mathrm{Cl}_{2}$ using a quartz cell with a path length of 1 $\mathrm{cm}$. For each compound, the mass of sample and the volume of solvent were both recorded and used to determine extinction coefficients through use of the Beer-Lambert law. ${ }^{7}$ Noise in the spectra within the high energy region $(<235 \mathrm{~nm})$ is related to the UV-cutoff of our solvent.

\begin{tabular}{|c|c|}
\hline Compound & $\lambda_{\max } / \mathrm{nm}\left(\varepsilon / \mathrm{M}^{-1} \mathrm{~cm}^{-1}\right)$ \\
\hline 3aa & 250 sh (23490), 261 (25855), 286 (20715), 311 (19681), 367 sh (3680), 455 (1144) \\
\hline $3 a b$ & 261 (28771), 286 (24181), 312 (22584), 367 sh (3608), 455 (1167) \\
\hline 3ac & $\begin{array}{c}260 \text { (20393), } 286 \text { sh (26634), } 297 \text { (27629), } 304 \text { (26573), } 325 \text { (24941), } 376 \text { sh (1870), } \\
456 \text { (887) }\end{array}$ \\
\hline 3ad & 259 (34159), 289 sh (18740), 309 (19655), 368 sh (2540), 456 (952) \\
\hline $3 \mathbf{b b}$ & 259 (34348), 287 sh (28687), 309 (27825), 364 sh (3444), 454 (1131) \\
\hline $3 b c$ & $264 \operatorname{sh}(21239), 293$ (26308), 314 (25405), 454 (861) \\
\hline 3bd & 257 (35155), 289 sh (20202), 308 (21015), 368 sh (2116), 454 (885) \\
\hline $3 c c$ & 280 sh (31941), 304 (38705), 324 sh (34752), 452 (1570) \\
\hline $3 \mathrm{~cd}$ & 259 (28172), 296 (27258), 316 (26560), 365 sh (1998), 451 (830) \\
\hline 3dd & 256 (41138), 307 (19837), 364 sh (1760), 450 (643) \\
\hline $4 a$ & 244 (17512), 261 sh (16494), 295 sh (14263), 305 (16024), 359 (2596), 460 (787) \\
\hline 4c & 274 sh (23581), 298 sh (31401), 312 (31965), 355 sh (2750), 445 (885) \\
\hline $6 a$ & 256 sh (30784), 309 (24310), 362 sh (4986), 456 (1991) \\
\hline $6 c$ & 288 sh (31441), 311 (33688), 367 sh (5687), 455 (1721) \\
\hline 7 aa & $247 \operatorname{sh}(26500), 260$ (27401), 296 sh (22112), 310 (23669), 367 sh (6163), 460 (2338) \\
\hline $7 a b$ & 254 (36721), 292 sh (29928), 308 (31182), 367 sh (6565), 460 (2356) \\
\hline $7 a c$ & $256 \operatorname{sh}(28040), 288 \operatorname{sh}(33086), 310(35766), 364 \operatorname{sh}(6546), 456$ (2459) \\
\hline $7 c c$ & $292 \operatorname{sh}(47466), 314$ (51250), $362 \operatorname{sh}(7828), 454$ (2256) \\
\hline
\end{tabular}


Supporting Information

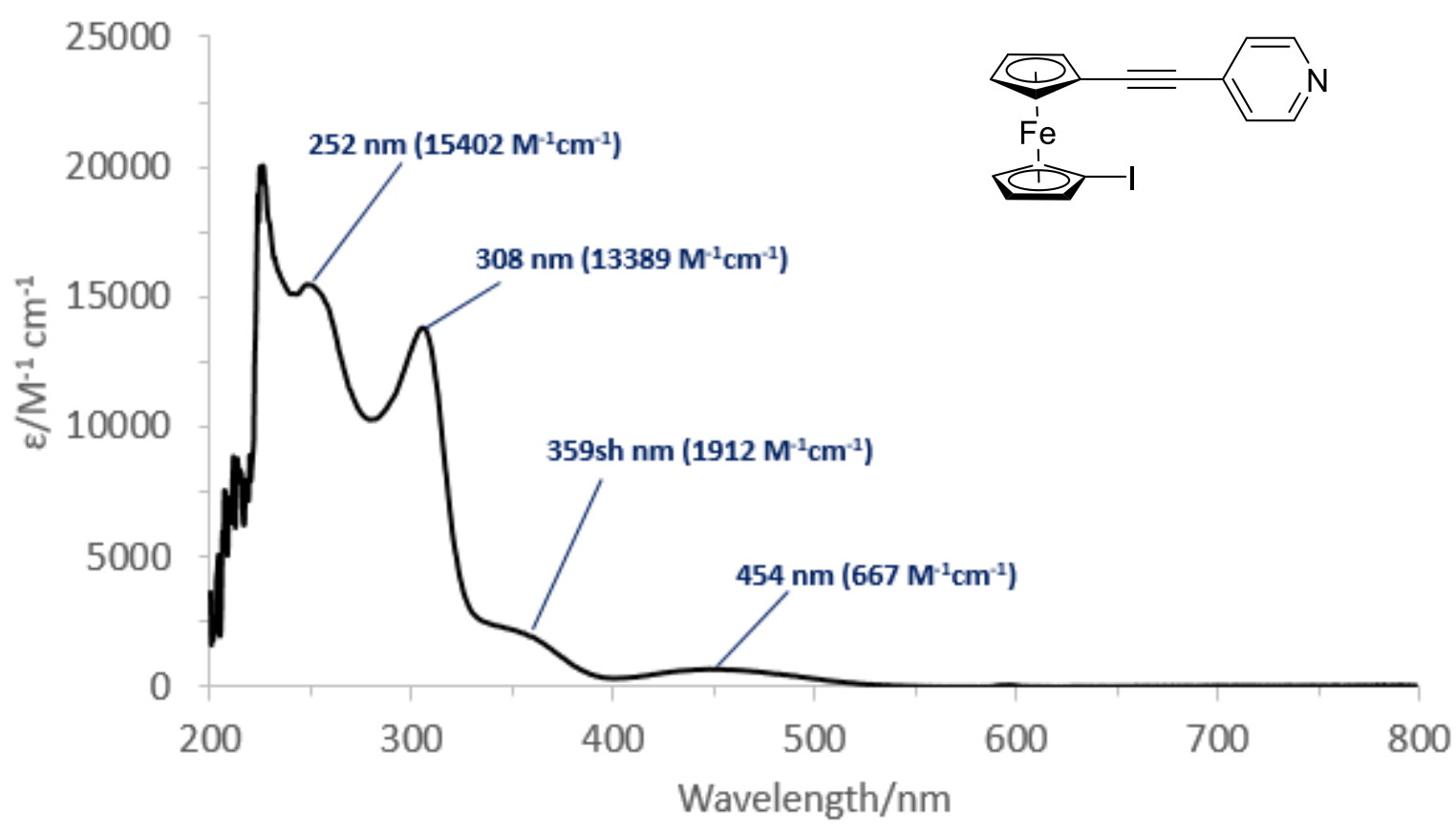

Figure S62: Annotated UV/Vis spectrum of compound 2a, structure inset.

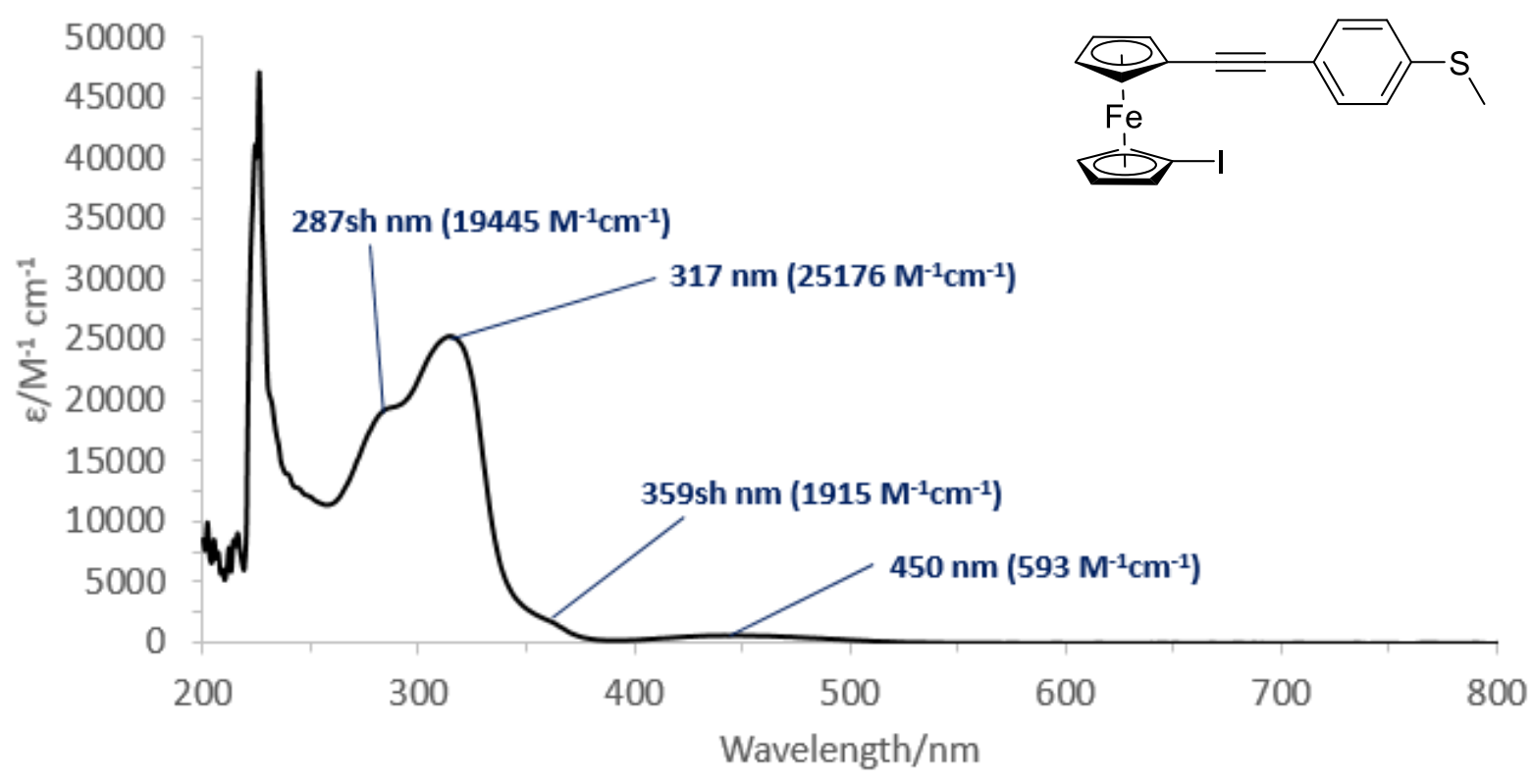

Figure S63: Annotated UV/Vis spectrum of compound 2c, structure inset. 


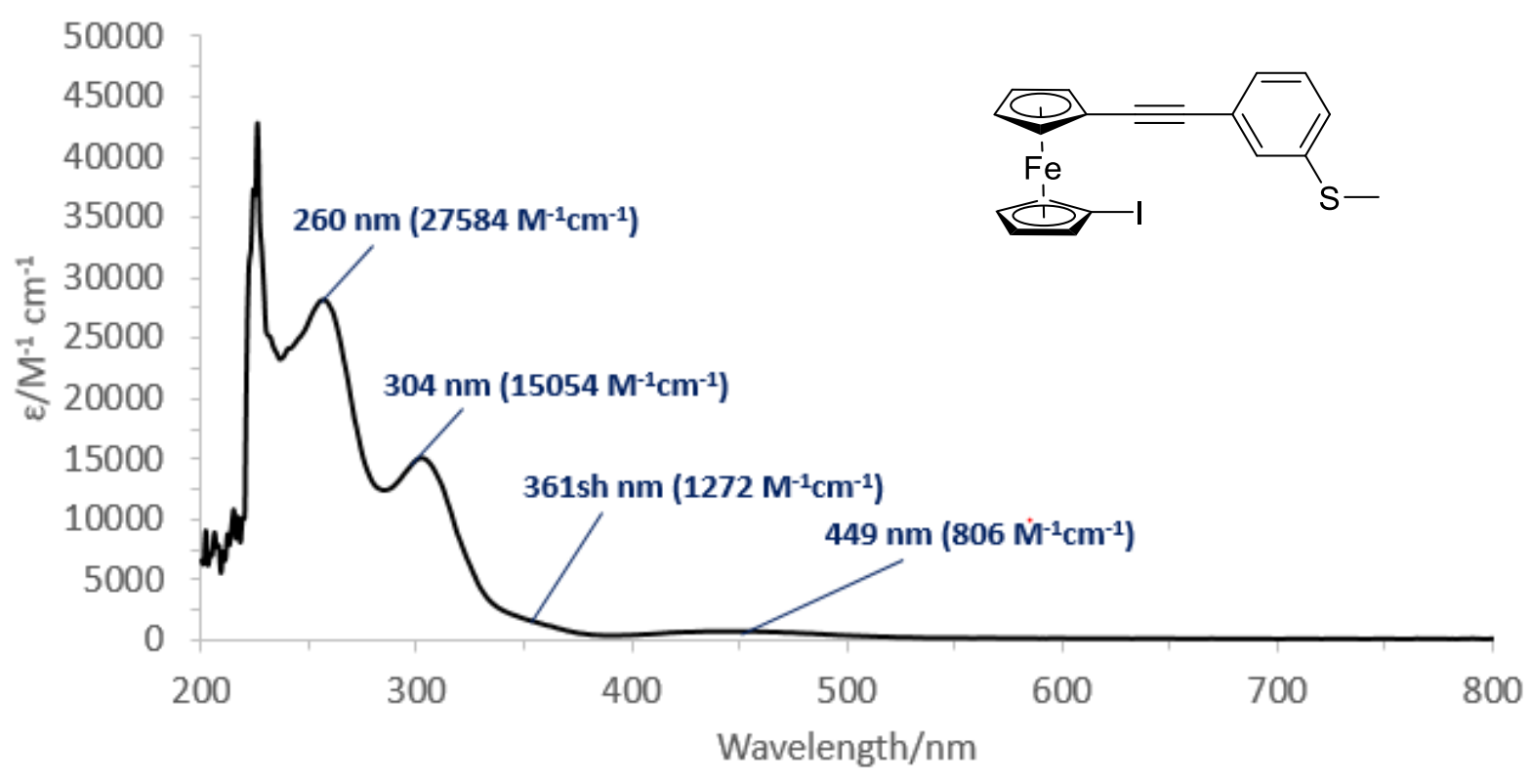

Figure S64: Annotated UV/Vis spectrum of compound 2d, structure inset.

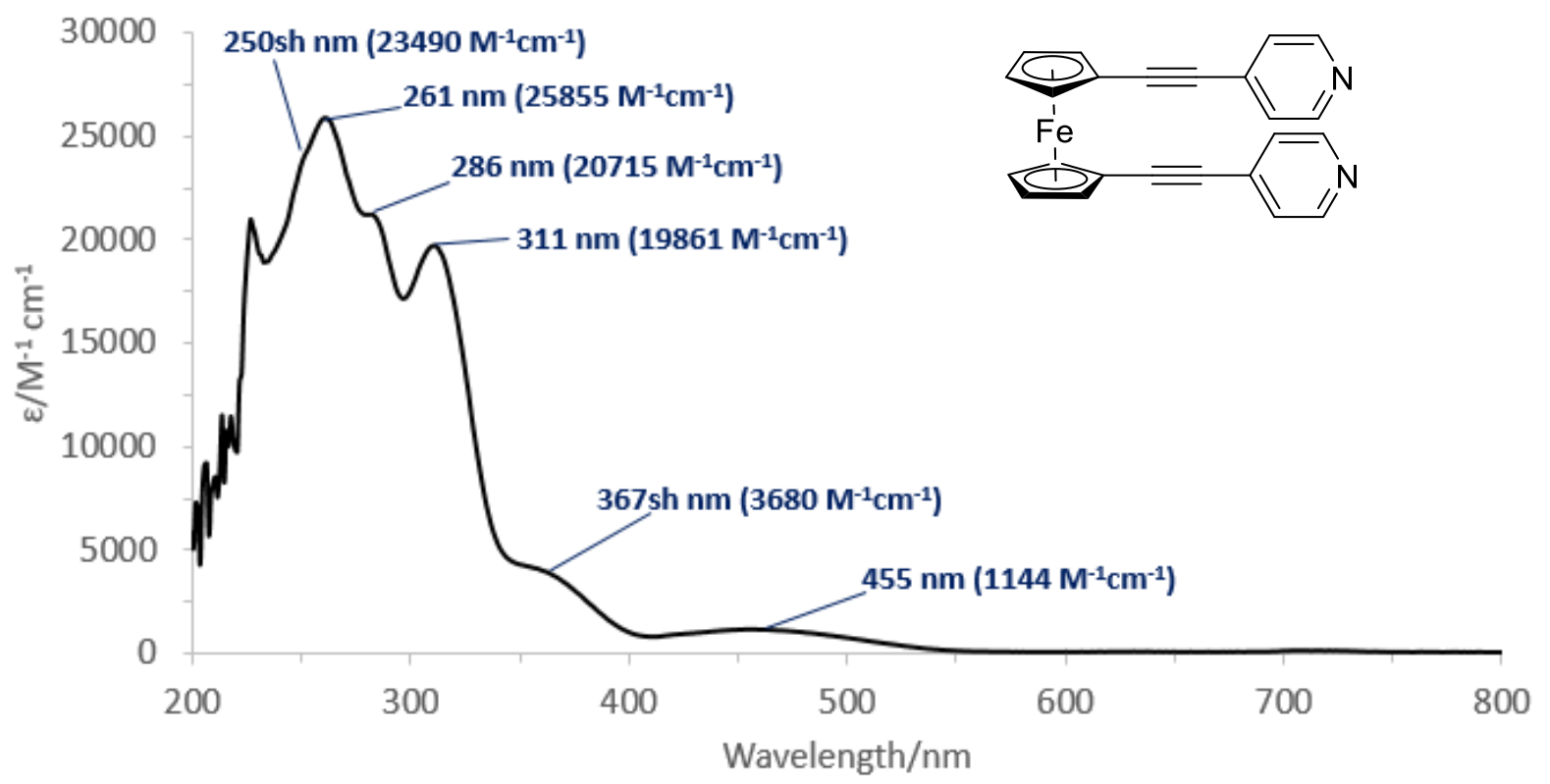

Figure S65: Annotated UV/Vis spectrum of compound 3aa, structure inset. 


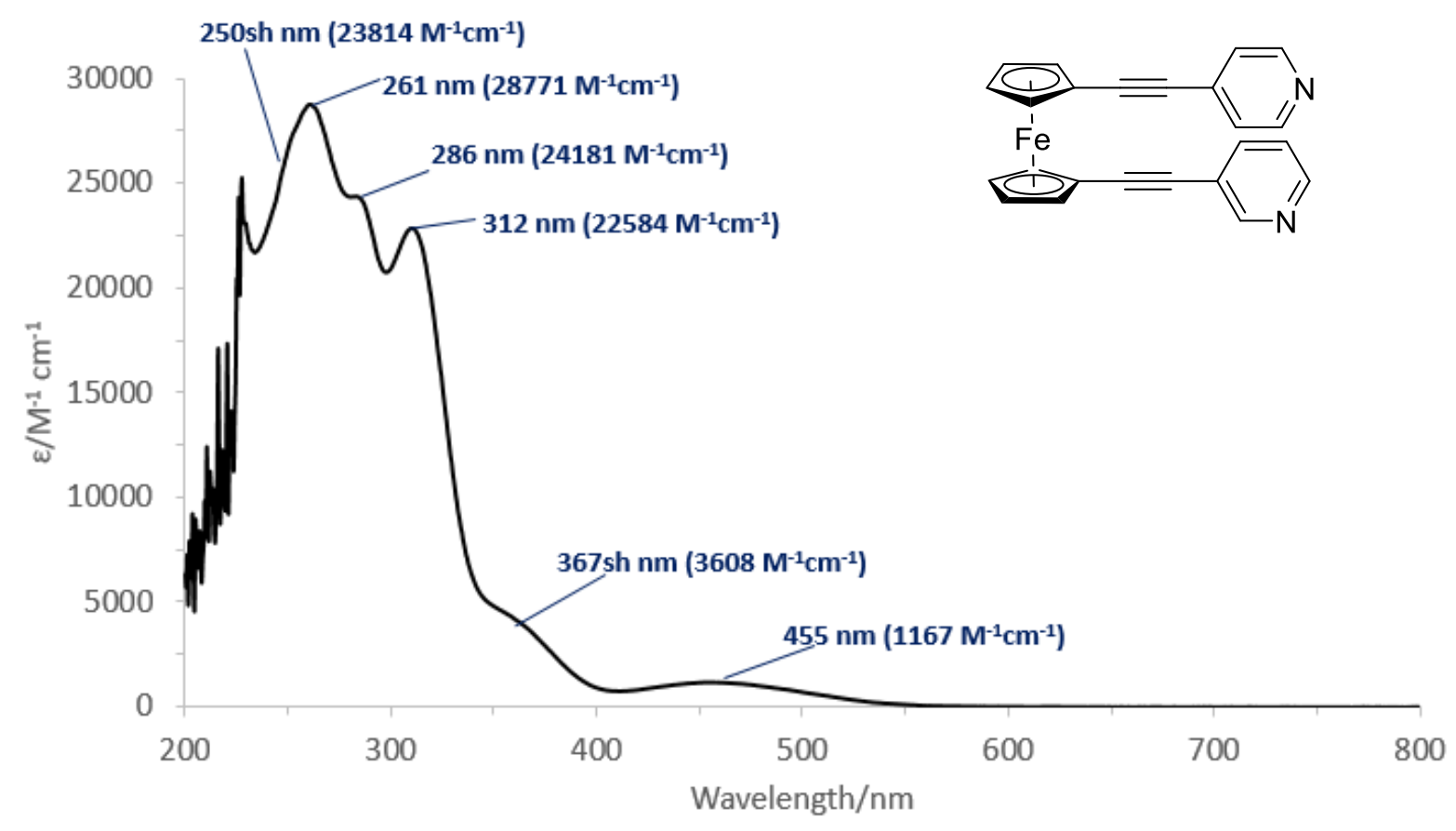

Figure S66: Annotated UV/Vis spectrum of compound 3ab, structure inset.

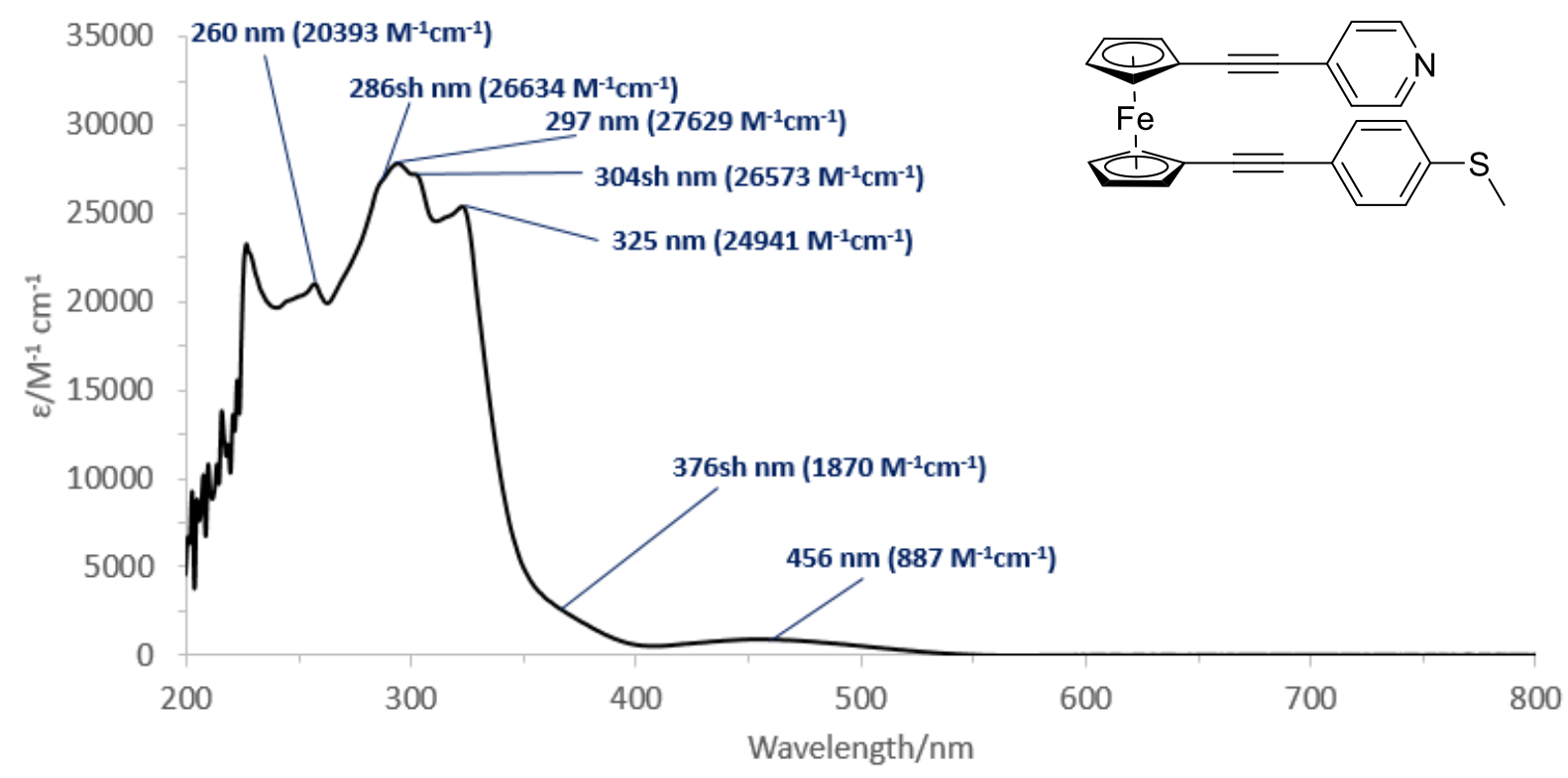

Figure S67: Annotated UV/Vis spectrum of compound 3ac, structure inset. 


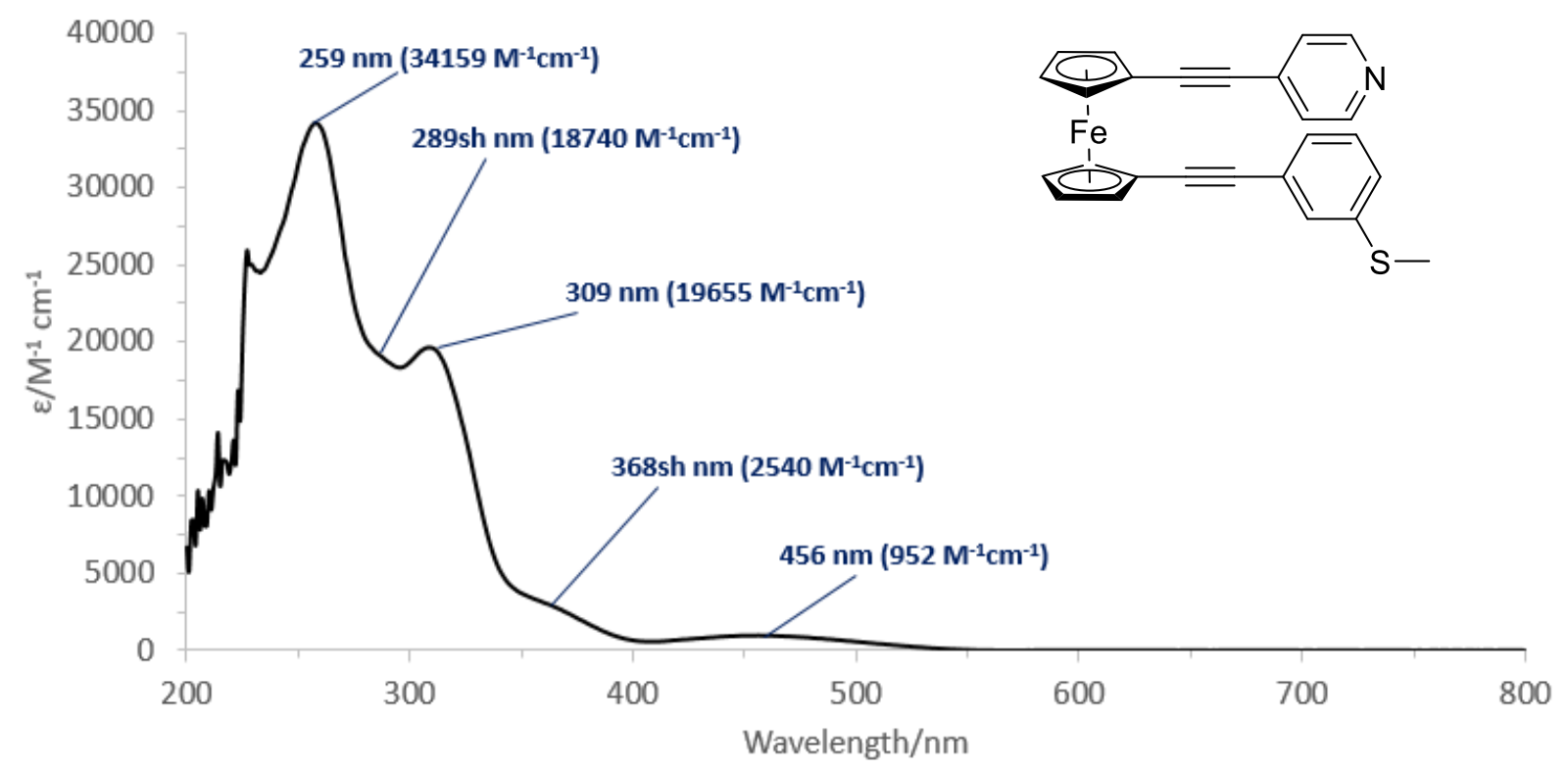

Figure S68: Annotated UV/Vis spectrum of compound 3ad, structure inset.

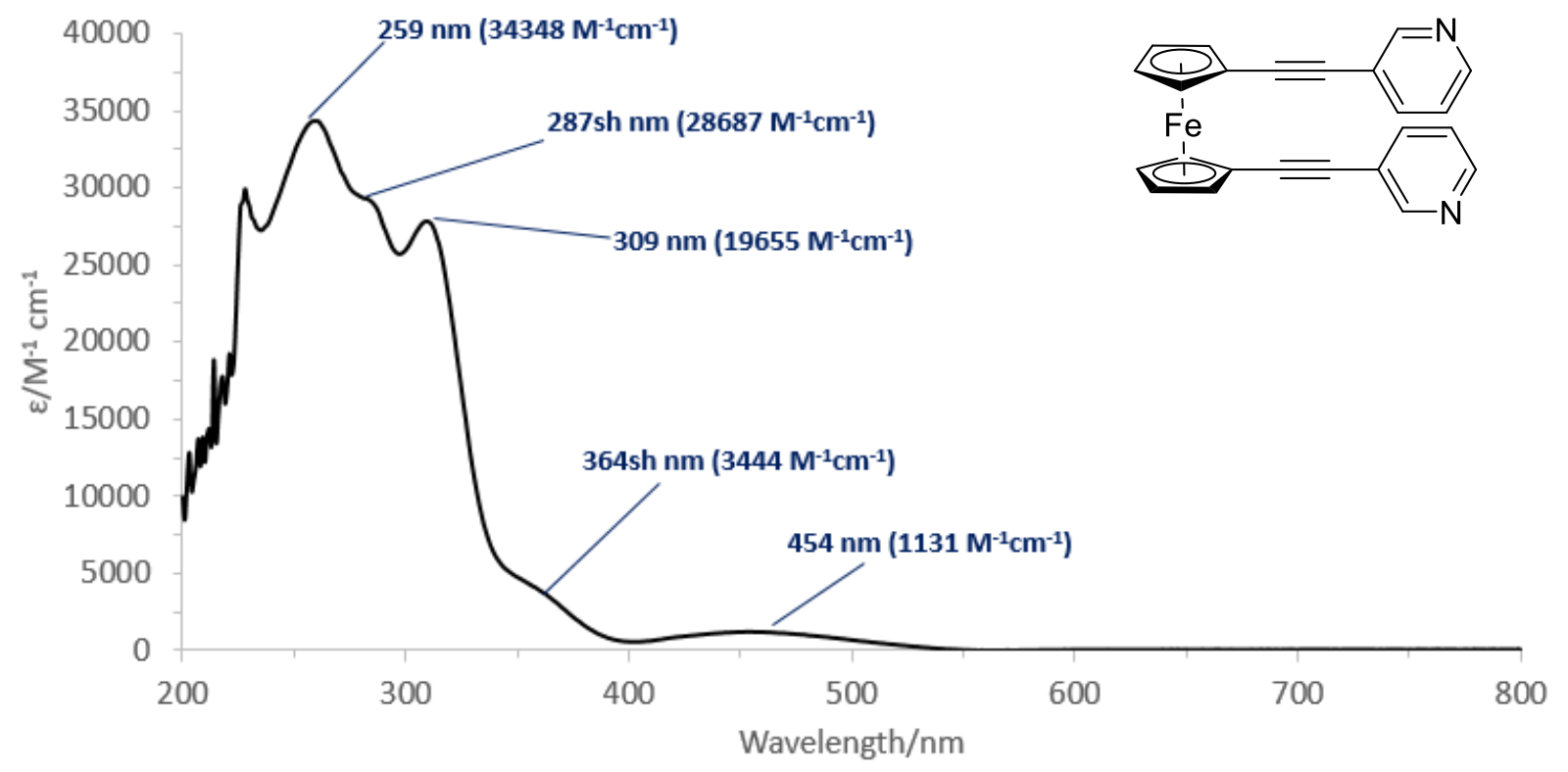

Figure S69: Annotated UV/Vis spectrum of compound $\mathbf{3 b b}$, structure inset. 
Supporting Information

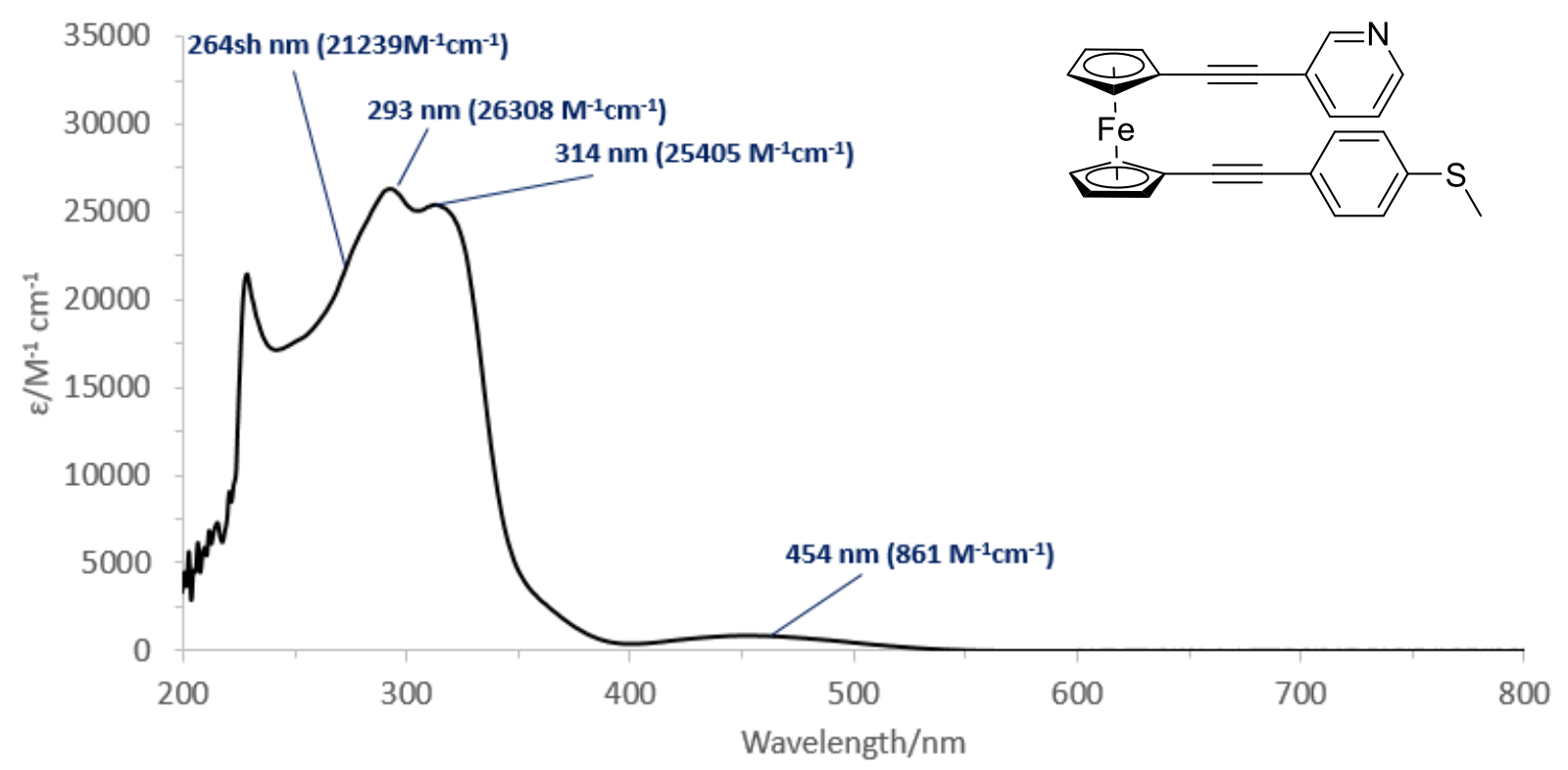

Figure S70: Annotated UV/Vis spectrum of compound 3bc, structure inset.

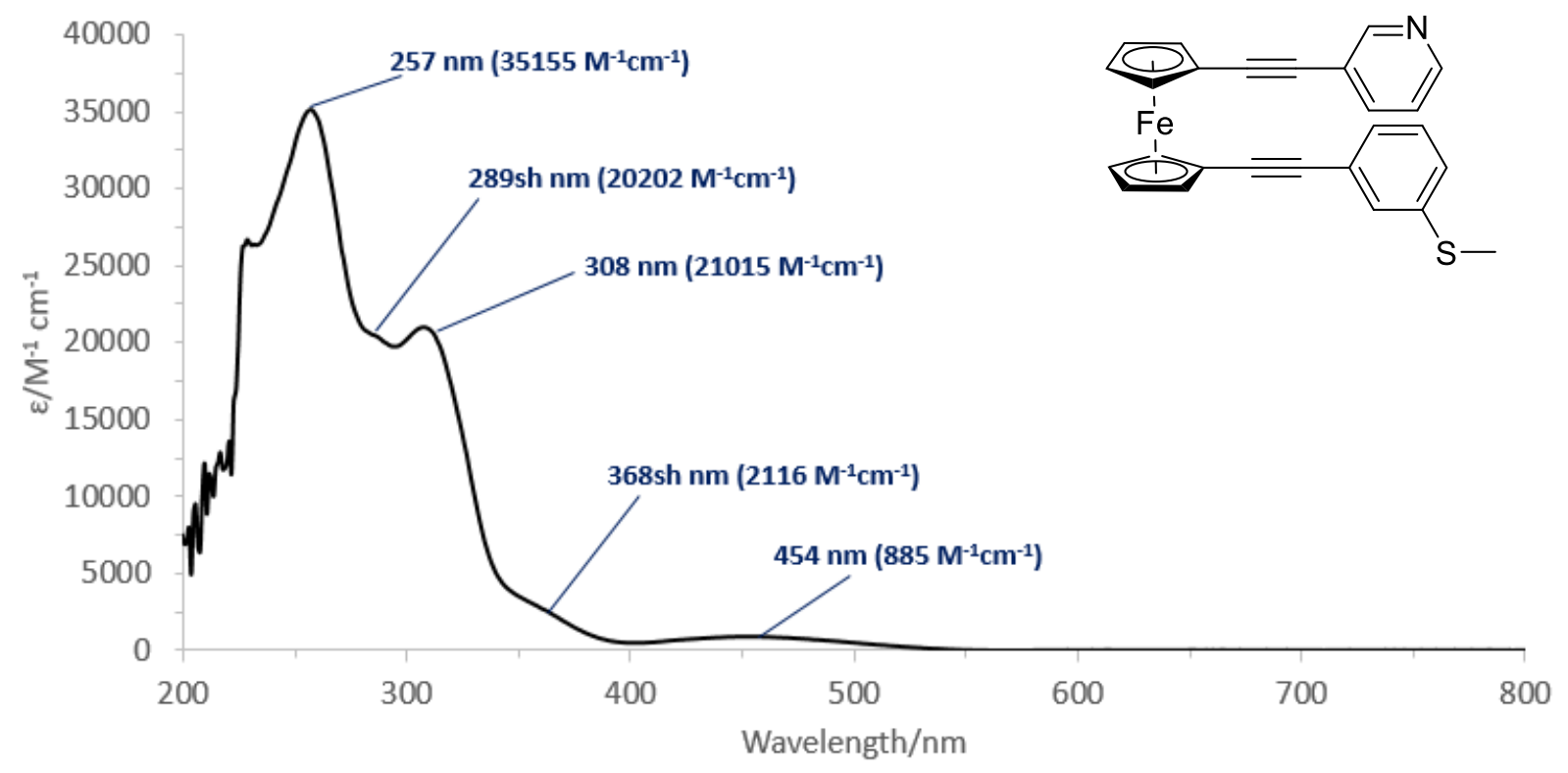

Figure S71: Annotated UV/Vis spectrum of compound 3bd, structure inset. 
Supporting Information

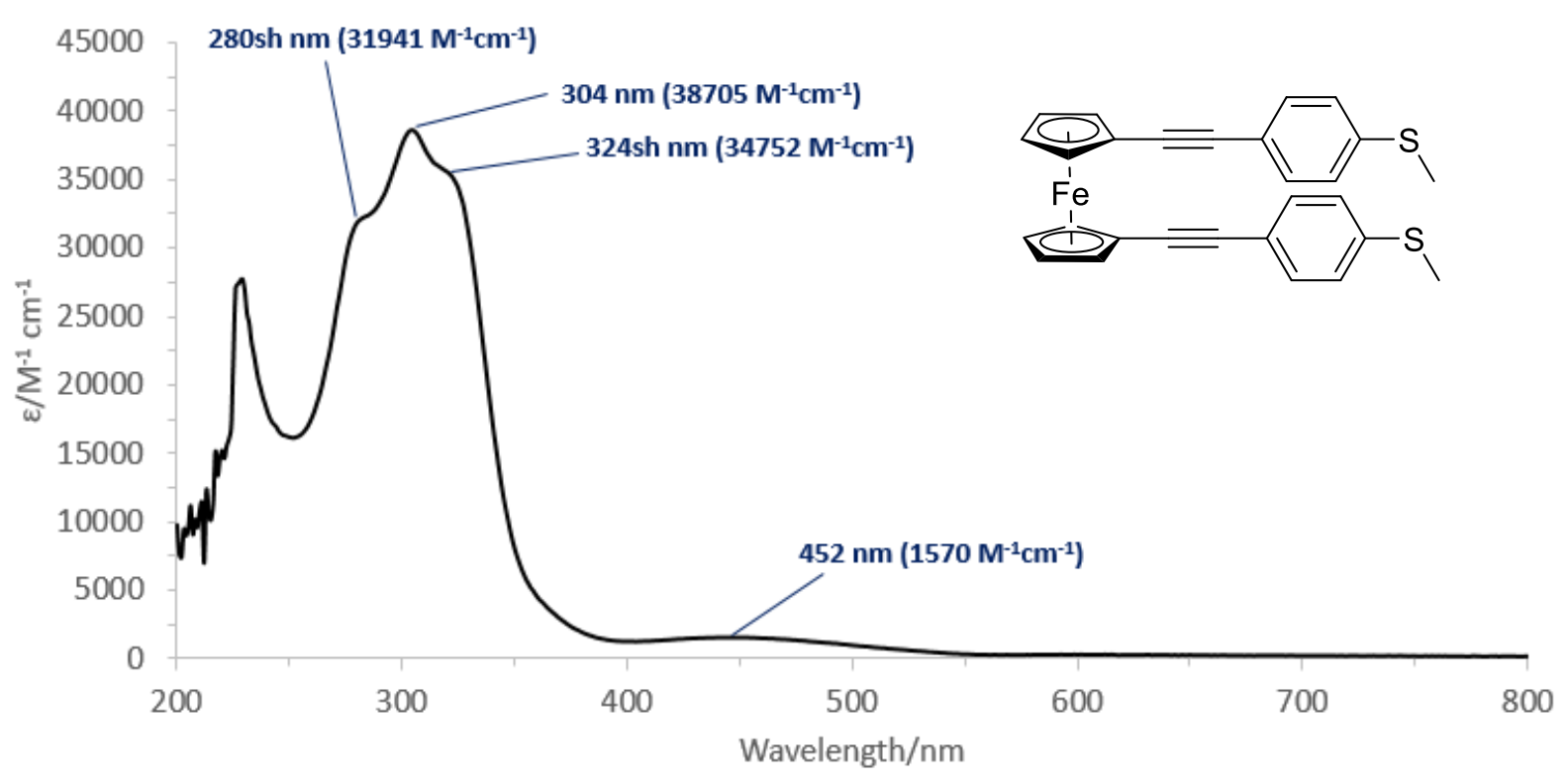

Figure S72: Annotated UV/Vis spectrum of compound 3cc, structure inset.

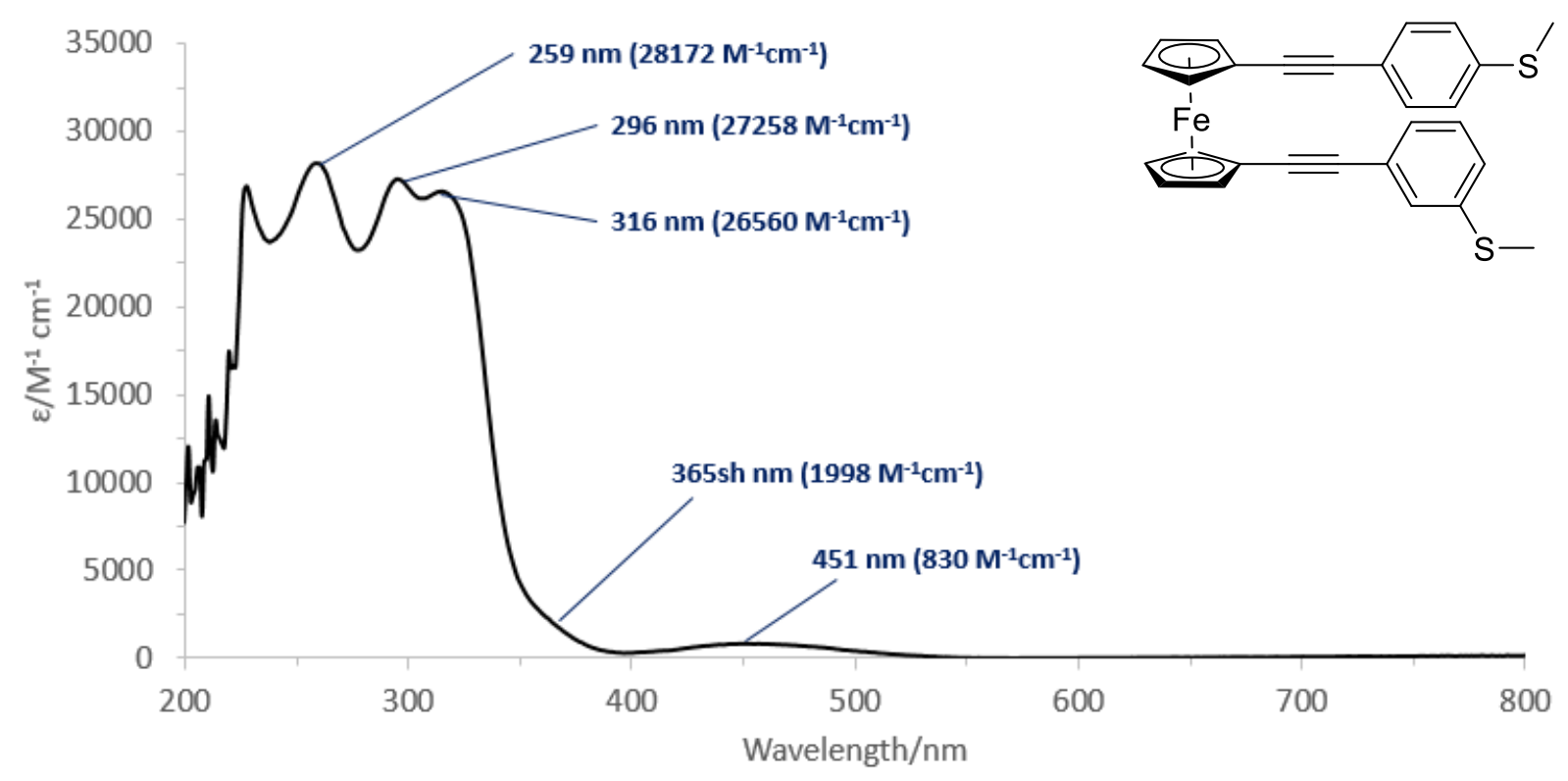

Figure S73: Annotated UV/Vis spectrum of compound 3cd, structure inset. 


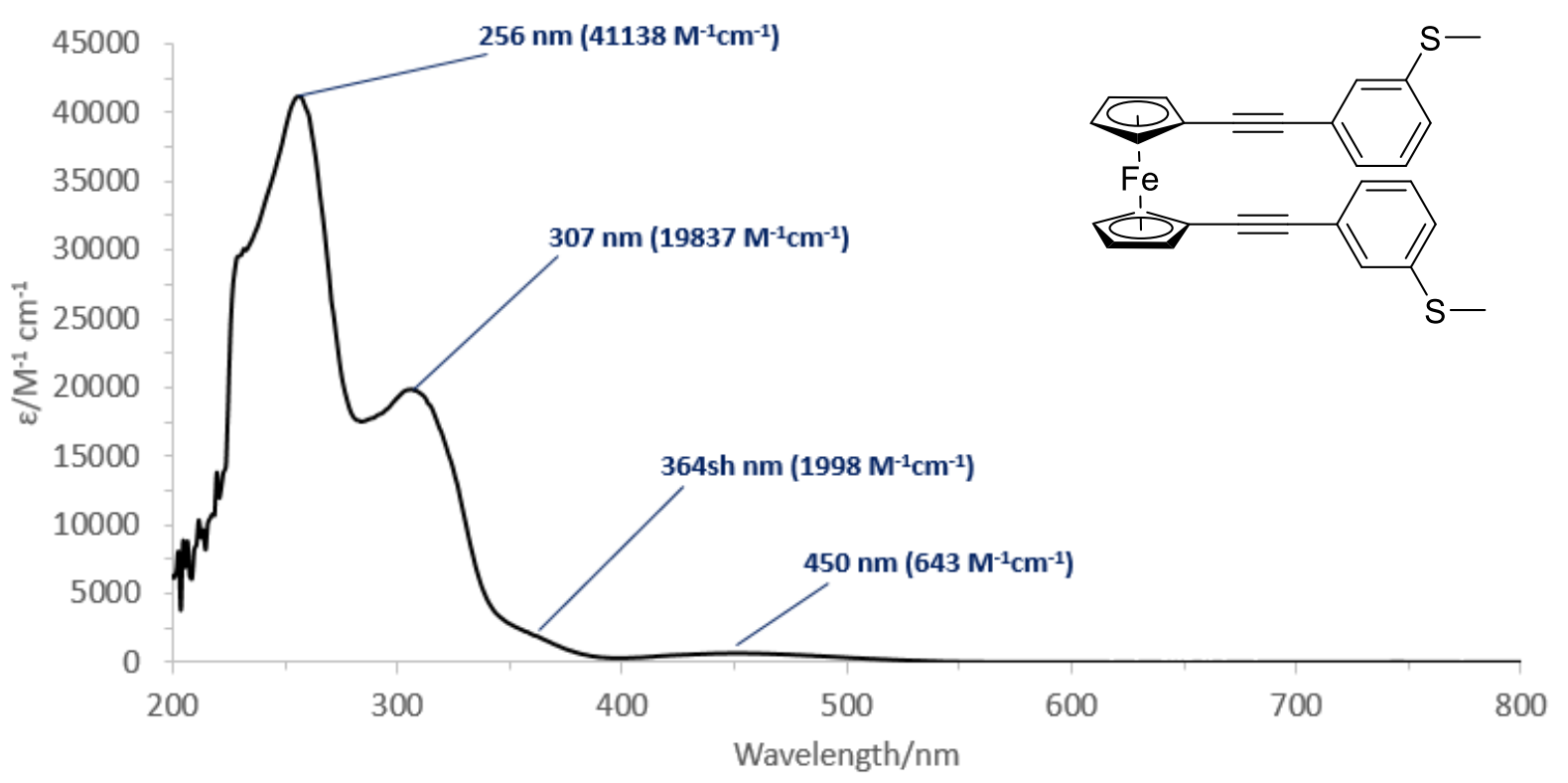

Figure S74: Annotated UV/Vis spectrum of compound 3dd, structure inset.

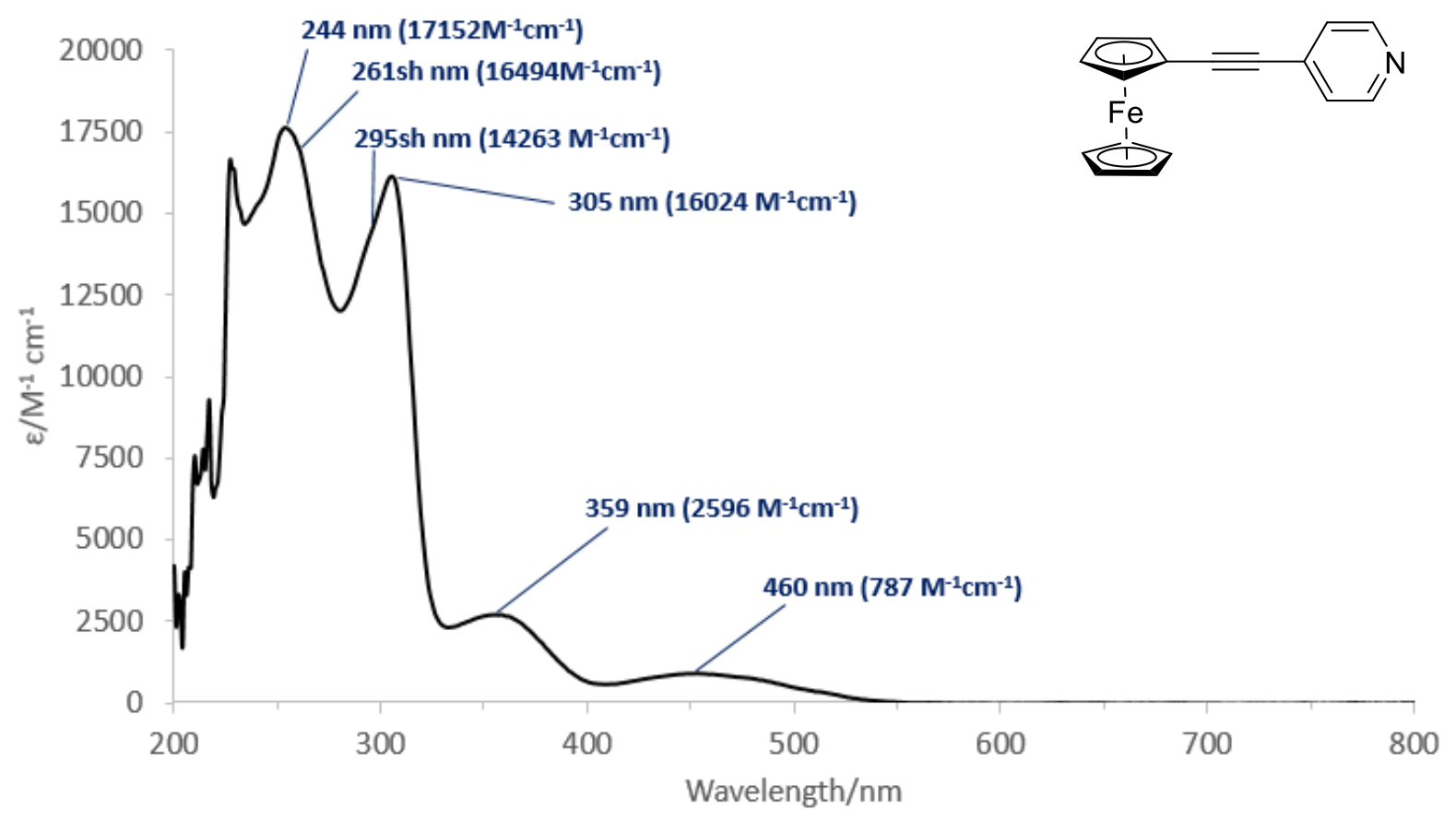

Figure S75: Annotated UV/Vis spectrum of compound 4a, structure inset. 


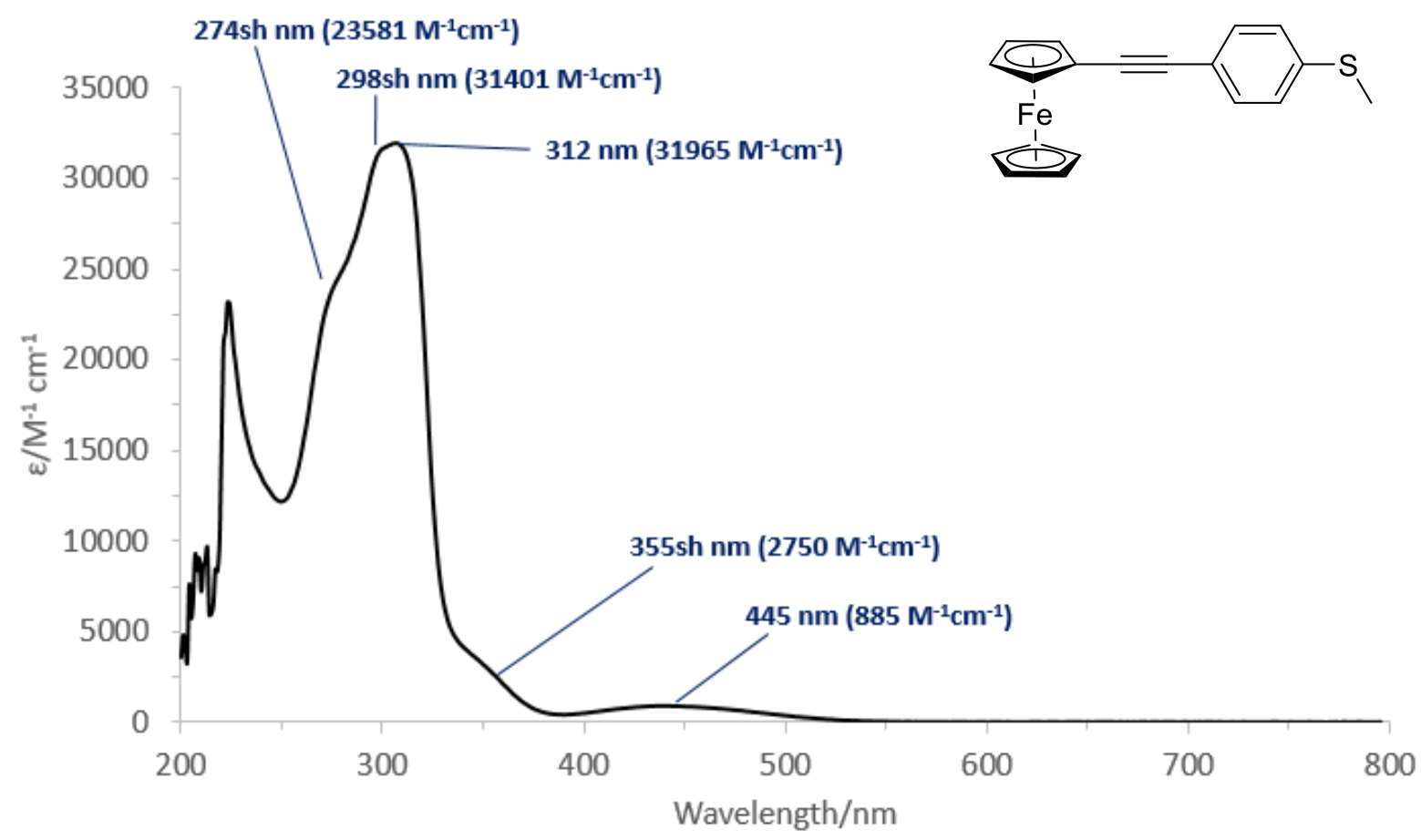

Figure S76: Annotated UV/Vis spectrum of compound 4c, structure inset.

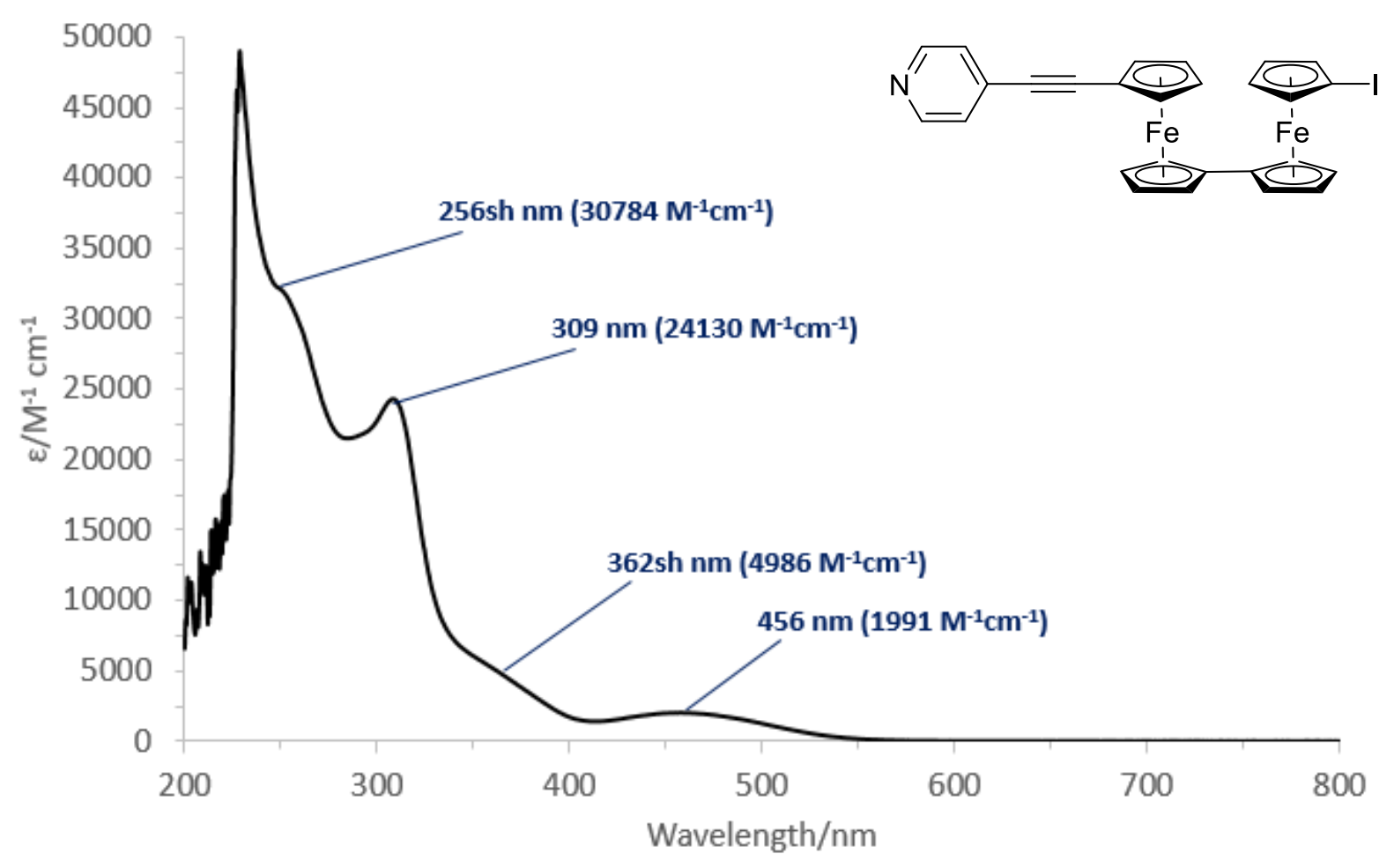

Figure S77: Annotated UV/Vis spectrum of compound $\mathbf{6 a}$, structure inset. 
Supporting Information

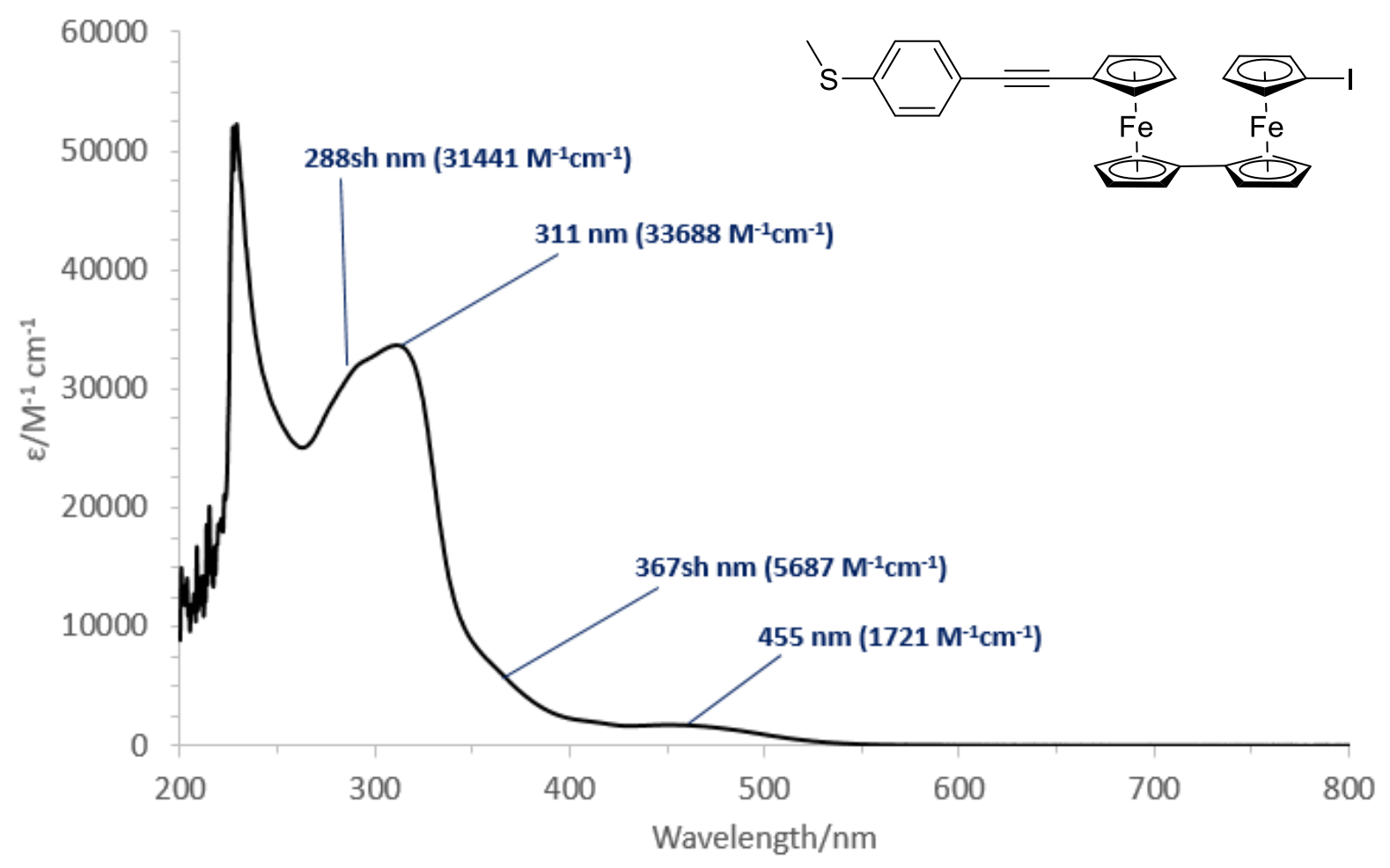

Figure S78: Annotated UV/Vis spectrum of compound $\mathbf{6 c}$, structure inset.

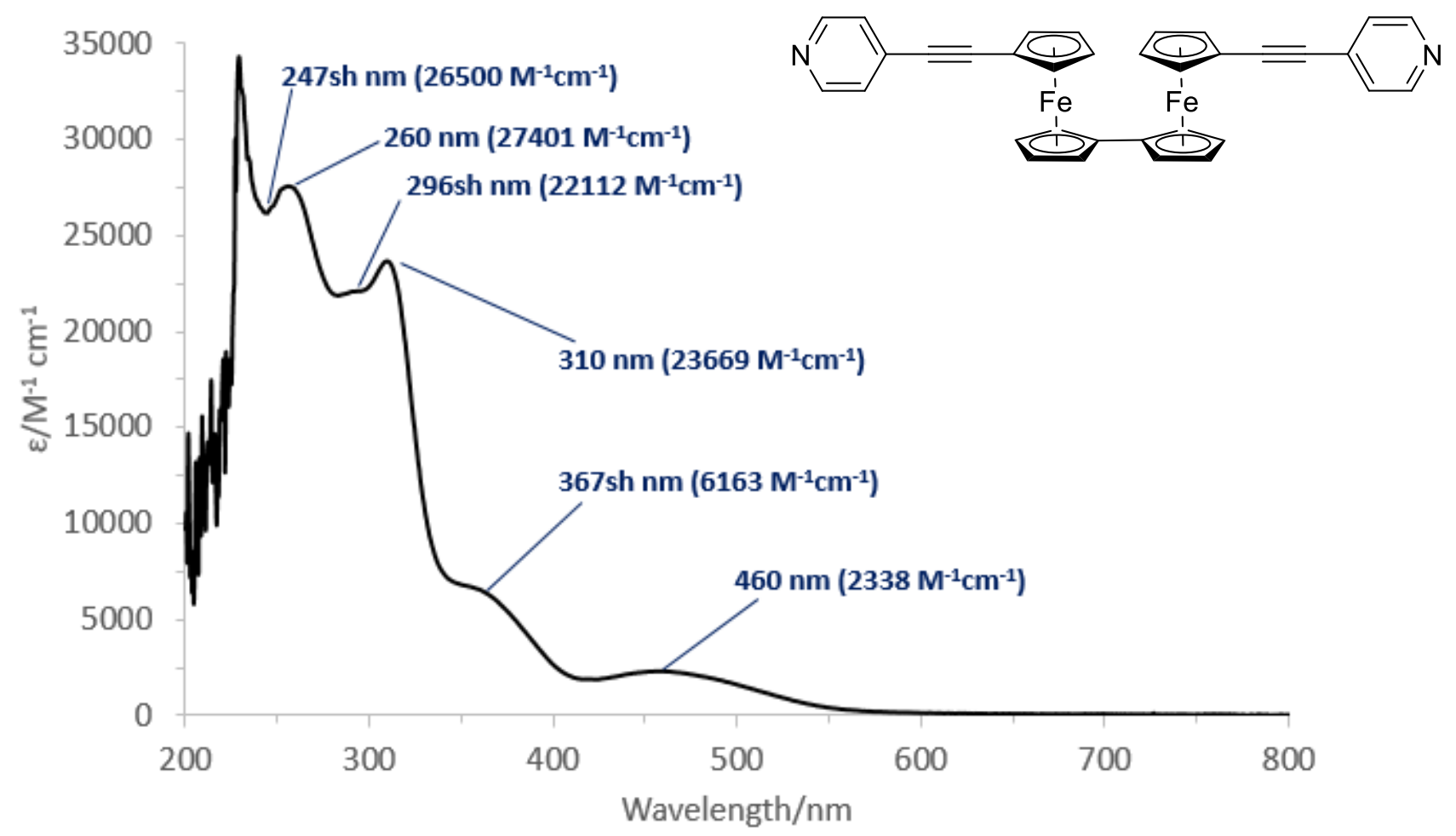

Figure S79: Annotated UV/Vis spectrum of compound 7aa, structure inset. 
Supporting Information

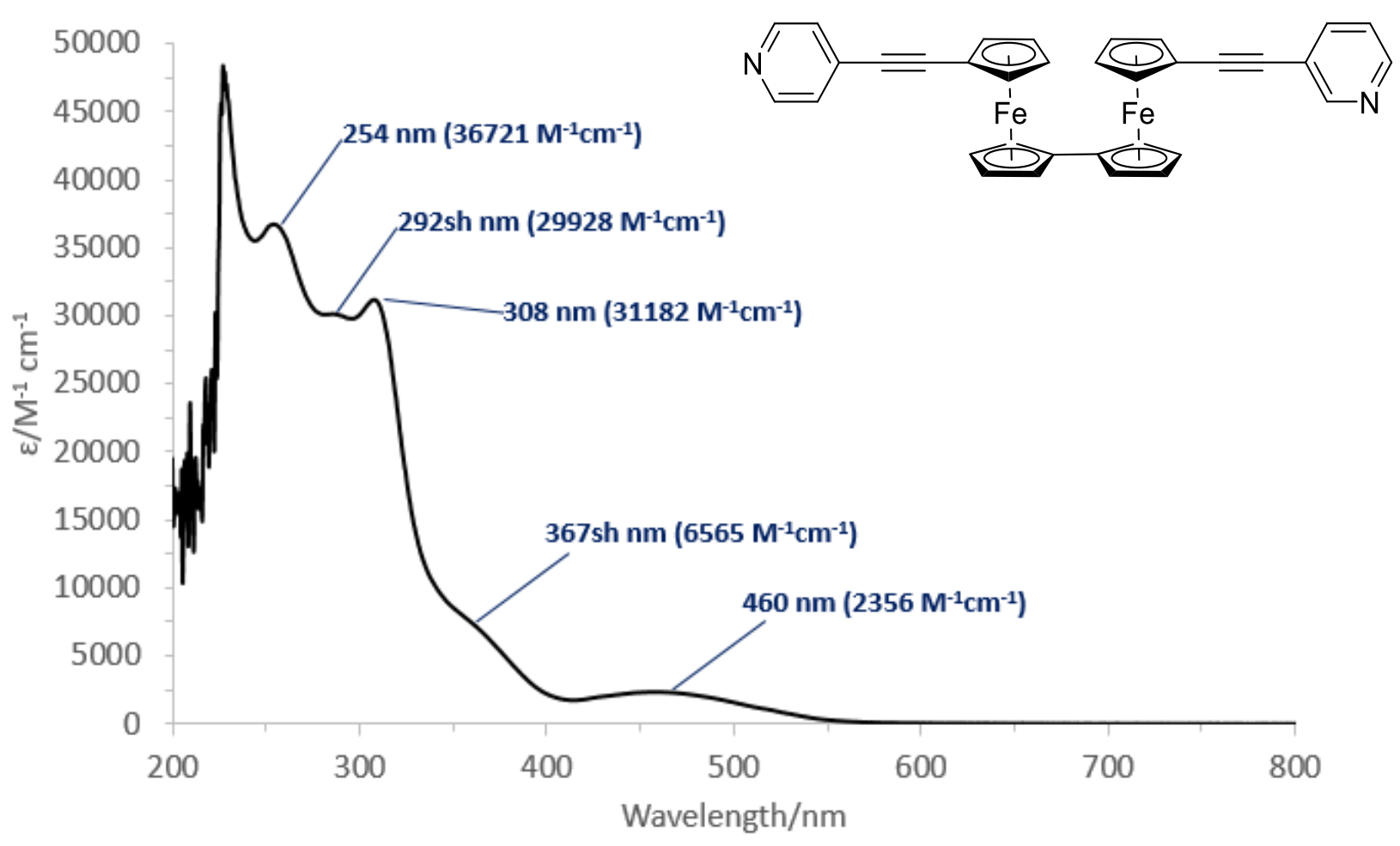

Figure S80: Annotated UV/Vis spectrum of compound 7ab, structure inset.

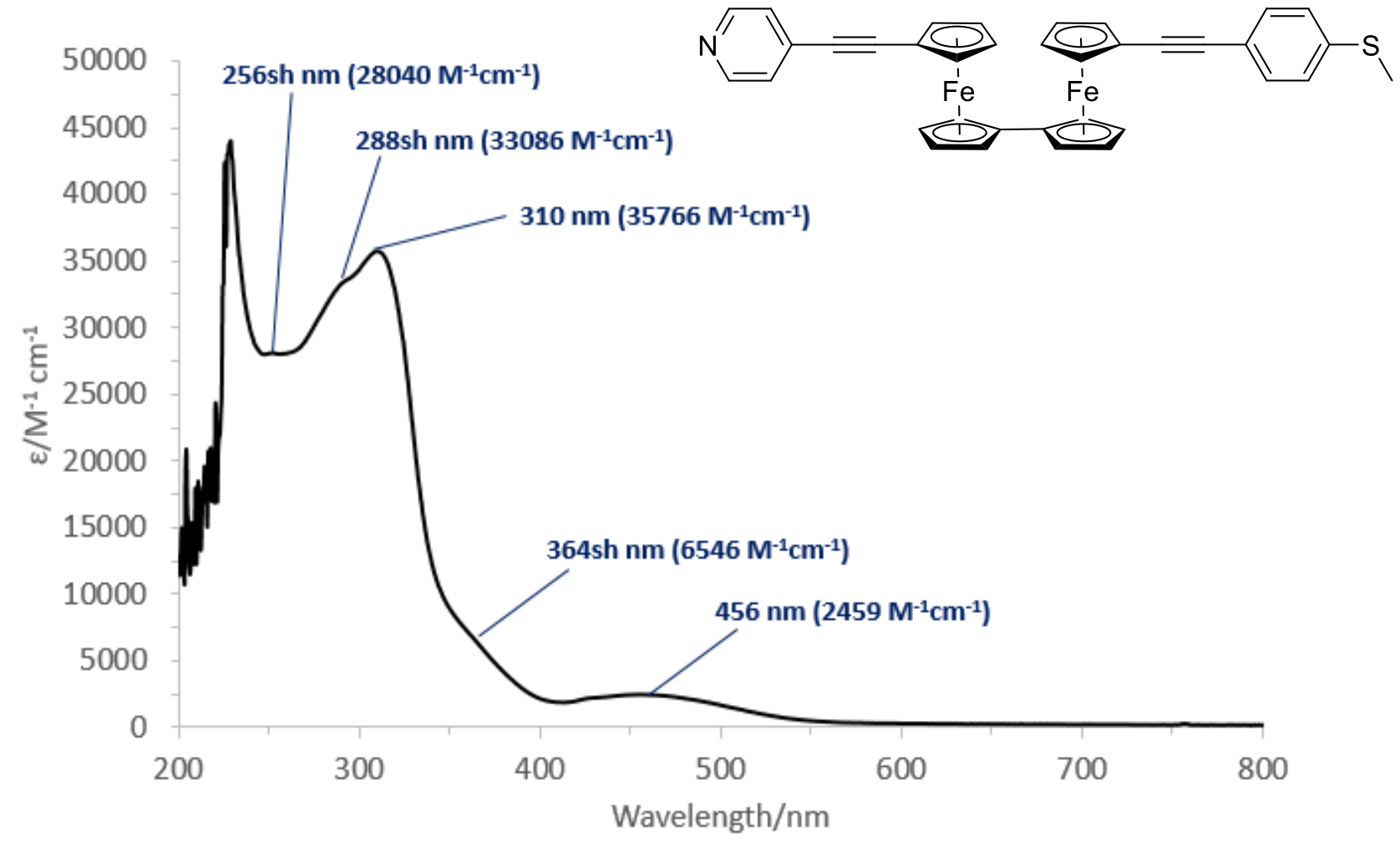

Figure S81: Annotated UV/Vis spectrum of compound 7ac, structure inset. 
Supporting Information

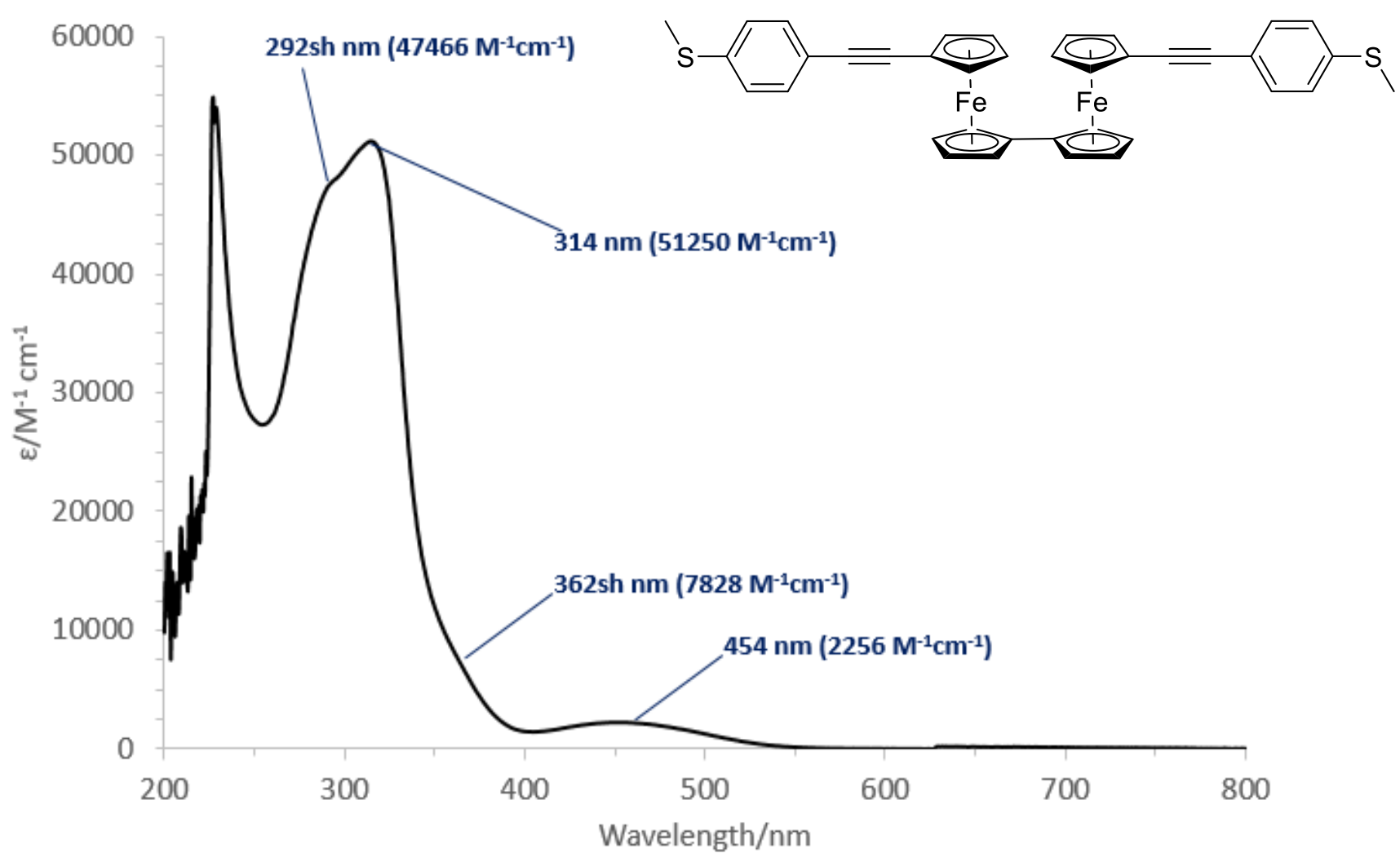

Figure S82: Annotated UV/Vis spectrum of compound 7cc, structure inset. 


\section{Computational Data}

\subsection{Computational methodology}

All calculations were performed using the Gaussian 16 suite ${ }^{8}$ with images generated in GaussView 6.0. ${ }^{9}$

Geometry optimisation of all compounds was performed in the gas phase using the B3LYP functional and the $6-311+G(d, p)$ basis set. Stationary points were confirmed by a lack of imaginary vibrations in the simulated infrared spectrum.

TD-DFT calculations were performed using a dichloromethane conductor-like solvent polarisation continuum model (CPCM) with the PBE1 functional and $6-31+G(d, p)$ basis set. 


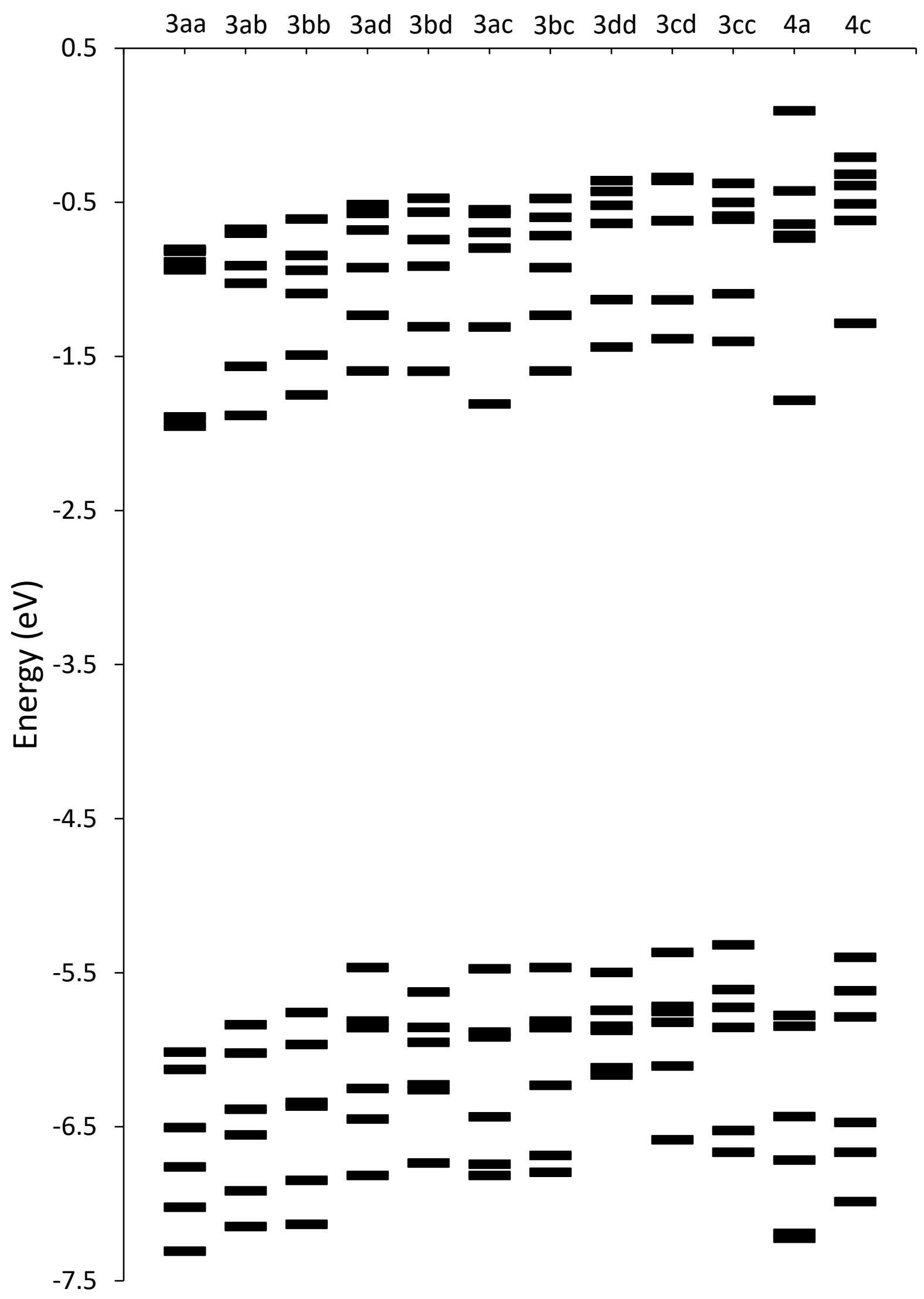

Figure S83: Energy level diagram for the frontier orbitals (HOMO-5 to LUMO-5) for $\mathbf{3 x x}$ and $\mathbf{4 x}$. 


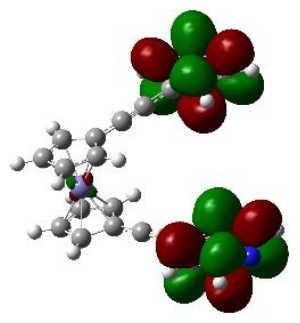

LUMO+5

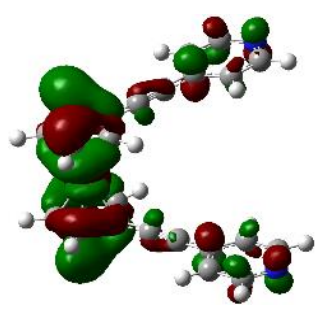

LUMO+2

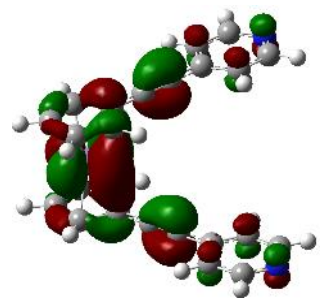

HOMO

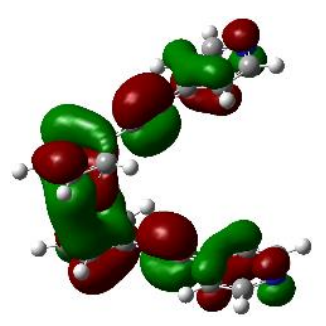

HOMO-3

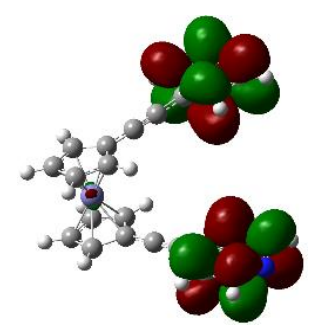

LUMO+4

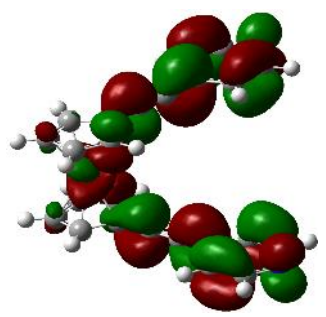

LUMO+1

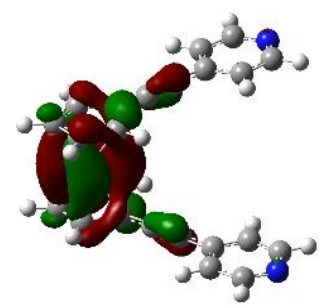

HOMO-1

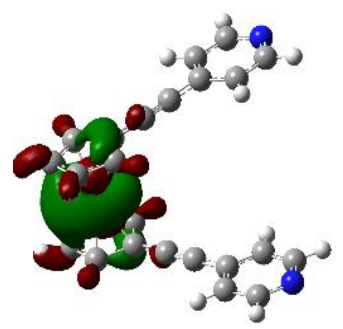

HOMO-4

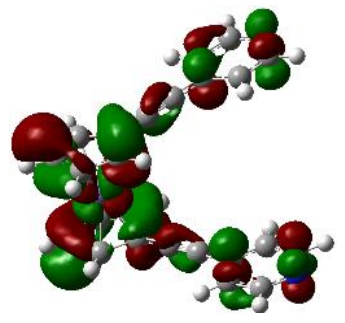

LUMO+3

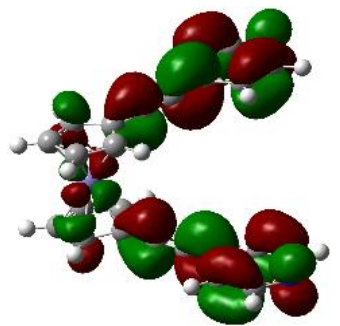

LUMO

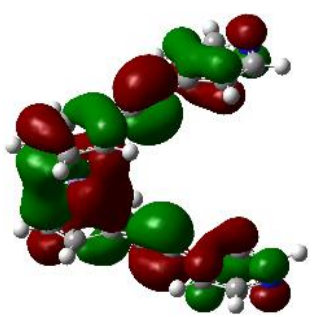

HOMO-2

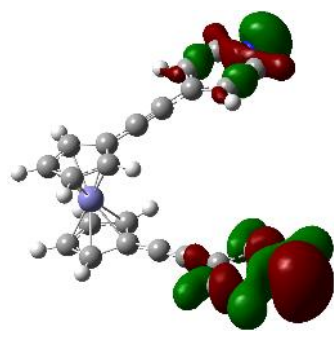

HOMO-5

Figure S84: Calculated iso-surfaces of HOMO-5 to LUMO-5 for compound 3aa

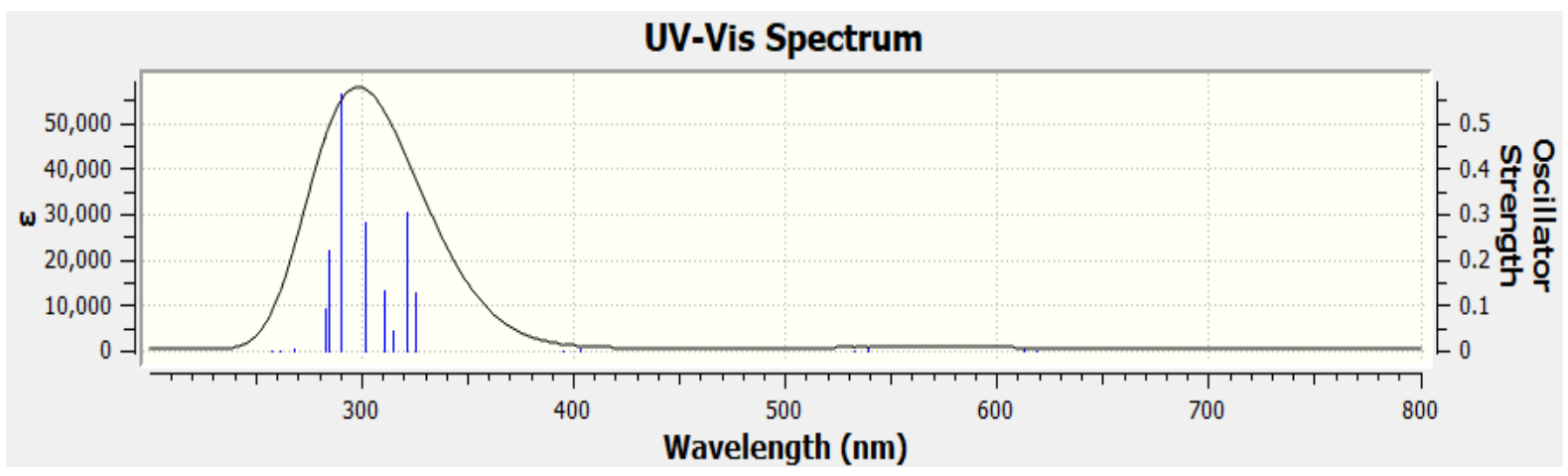

Figure S85: Calculated UV/Vis spectrum for compound 3aa 
Table S2: Assignment of transitions observed in the calculated UV/Vis spectra of compound 3aa

\begin{tabular}{|c|c|c|c|}
\hline $\begin{array}{c}\text { Wavelength } \\
(\mathrm{nm})\end{array}$ & $\begin{array}{l}\text { Oscillator } \\
\text { Strength } \\
\text { (f) }\end{array}$ & Assignment & $\begin{array}{l}\text { Excited } \\
\text { State }\end{array}$ \\
\hline 633.35 & 0.0039 & $\begin{array}{l}\mathrm{H}-2 \rightarrow \mathrm{L}(3.53 \%), \mathrm{H}-2 \rightarrow \mathrm{L}+5(5.50 \%) \\
\mathrm{H}-1 \rightarrow \mathrm{L}+4(18.36 \%), \mathrm{H} \rightarrow \mathrm{L}(27.77 \%) \\
H \rightarrow L+1(4.07 \%), H \rightarrow L+5(30.87 \%)\end{array}$ & 1 \\
\hline 404.83 & 0.0015 & $\begin{array}{l}H-4 \rightarrow L(16.62 \%), H-4 \rightarrow L+1(2.42 \%), \\
H-4 \rightarrow L+5(18.69 \%), H-2 \rightarrow L(4.49 \%), \\
H-2 \rightarrow L+5(3.54 \%), H-1 \rightarrow L+4 \\
(33.69 \%), H \rightarrow L(10.01 \%), H \rightarrow L+5 \\
(2.74 \%)\end{array}$ & 5 \\
\hline 383.53 & 0.0002 & $\begin{array}{l}H-4 \rightarrow \mathrm{L}+4(53.85 \%), H-1 \rightarrow \mathrm{L} \\
(12.20 \%), \mathrm{H}-1 \rightarrow \mathrm{L}+5(3.29 \%), \mathrm{H} \rightarrow \mathrm{L}+4 \\
(24.09 \%)\end{array}$ & 6 \\
\hline 335.05 & 0.0489 & $\begin{array}{l}\mathrm{H}-2 \rightarrow \mathrm{L}(3.77 \%), \mathrm{H}-1 \rightarrow \mathrm{L}(9.74 \%), \mathrm{H}- \\
1 \rightarrow \mathrm{L}+1(33.14 \%), \mathrm{H} \rightarrow \mathrm{L}(19.59 \%) \\
H \rightarrow L+5(19.55 \%)\end{array}$ & 7 \\
\hline 333.75 & 0.0771 & $\begin{array}{l}H-2 \rightarrow L(6.51 \%), H-1 \rightarrow L(8.42 \%), H- \\
1 \rightarrow L+1(37.49 \%), H \rightarrow L(19.34 \%) \\
H \rightarrow L+1(15.68 \%), H \rightarrow L+5(2.26 \%)\end{array}$ & 8 \\
\hline 319.27 & 0.1698 & $\begin{array}{l}H-3 \rightarrow L(12.31 \%), H-2 \rightarrow L(21.92 \%), \\
H \rightarrow L(9.22 \%), H \rightarrow L+1(42.37 \%) \\
H \rightarrow L+5(5.29 \%)\end{array}$ & 9 \\
\hline 295.95 & 0.0637 & $\begin{array}{l}\mathrm{H}-3 \rightarrow \mathrm{L}(9.45 \%), \mathrm{H}-2 \rightarrow \mathrm{L}(51.10 \%), \mathrm{H}- \\
2 \rightarrow \mathrm{L}+1(4.33 \%), \mathrm{H}-1 \rightarrow \mathrm{L}(3.16 \%), \mathrm{H} \rightarrow \\
\mathrm{L}(4.88 \%), \mathrm{H} \rightarrow \mathrm{L}+5(15.93 \%)\end{array}$ & 10 \\
\hline 293.16 & 0.0526 & $\begin{array}{l}\mathrm{H}-2 \rightarrow \mathrm{L}(3.45 \%), \mathrm{H}-1 \rightarrow \mathrm{L}(28.82 \%), \mathrm{H}- \\
1 \rightarrow \mathrm{L}+2(13.87 \%), \mathrm{H}-1 \rightarrow \mathrm{L}+5 \\
(38.48 \%)\end{array}$ & 11 \\
\hline 288.12 & 0.3829 & $\begin{array}{l}H-4 \rightarrow L(3.71 \%), H-3 \rightarrow L(55.70 \%), H- \\
2 \rightarrow L+1(5.69 \%), H \rightarrow L(2.51 \%) \\
H \rightarrow L+1(7.23 \%), H \rightarrow L+2(3.77 \%) \\
H \rightarrow L+5(13.21 \%)\end{array}$ & 12 \\
\hline 281.92 & 0.8789 & $\begin{array}{l}H-3 \rightarrow L(10.63 \%), H-2 \rightarrow L+1 \\
(75.24 \%), H \rightarrow L+5(3.69 \%)\end{array}$ & 13 \\
\hline 273.94 & 0.0747 & $\begin{array}{l}\mathrm{H}-3 \rightarrow \mathrm{L}+2(8.70 \%), \mathrm{H}-2 \rightarrow \mathrm{L}+2 \\
(6.63 \%), \mathrm{H} \rightarrow \mathrm{L}+2(71.91 \%), \mathrm{H} \rightarrow \mathrm{L}+5 \\
(3.69 \%)\end{array}$ & 14 \\
\hline
\end{tabular}




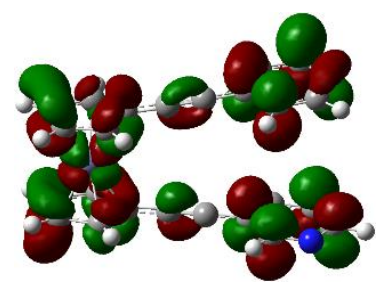

LUMO+5

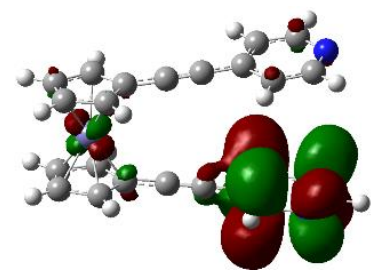

LUMO+2

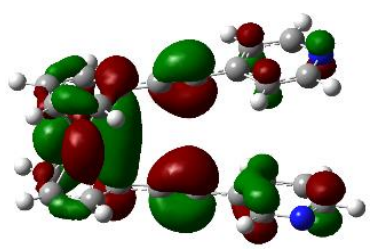

HOMO

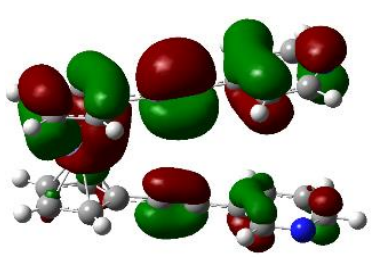

HOMO-3

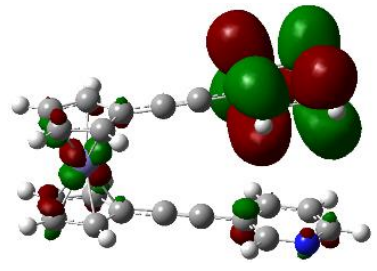

LUMO+4

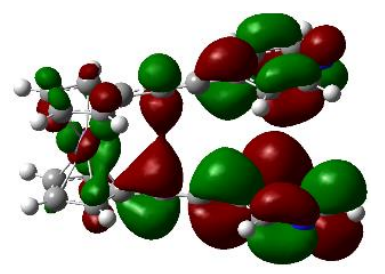

LUMO+1

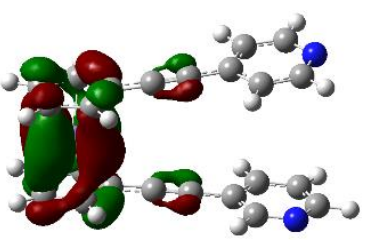

HOMO-1

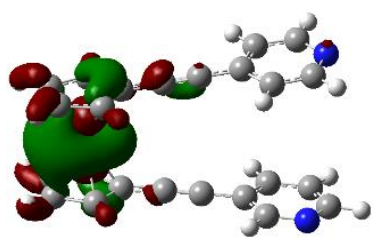

HOMO-4

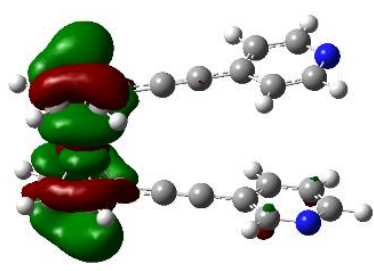

LUMO+3

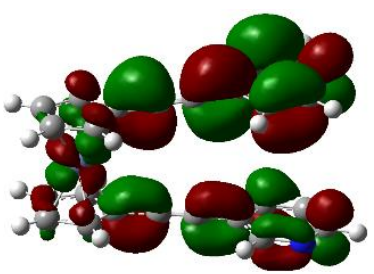

LUMO

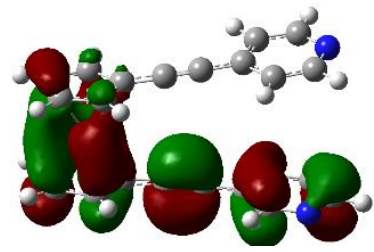

HOMO-2

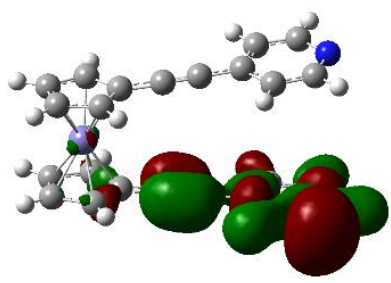

HOMO-5

Figure S86: Calculated iso-surfaces of HOMO-5 to LUMO-5 for compound 3ab

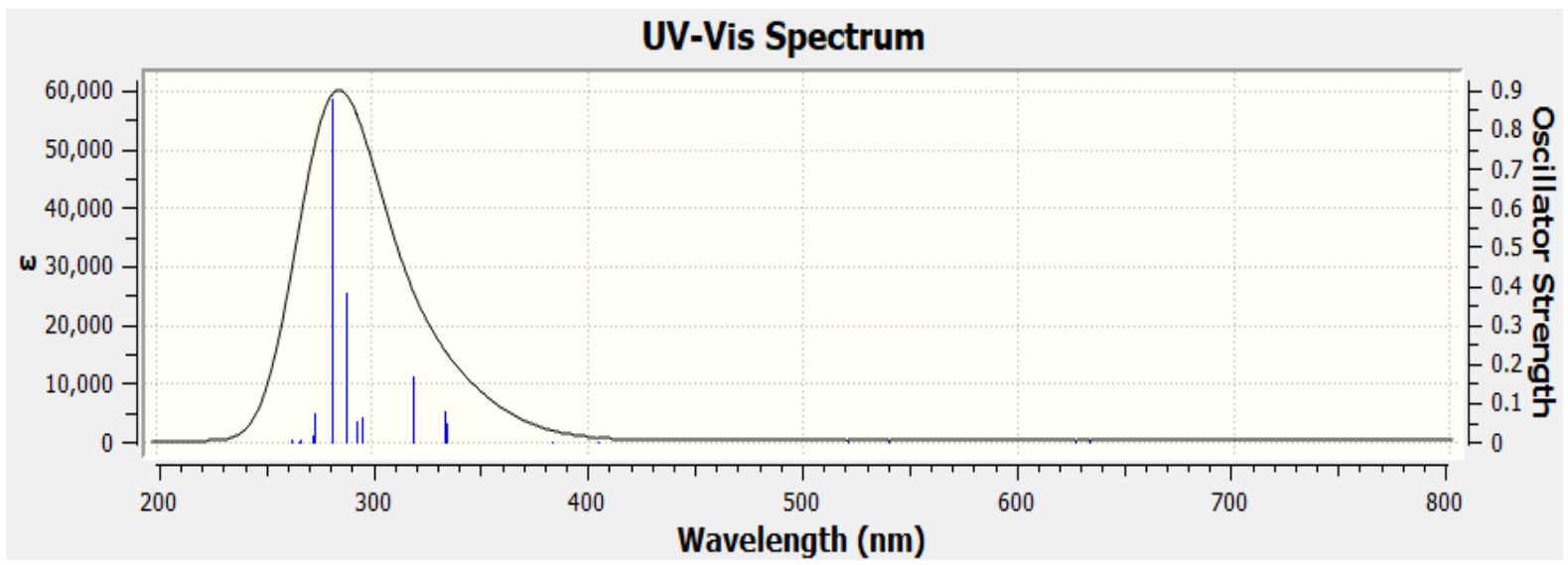

Figure S87: Calculated UV/Vis spectrum for compound 3ab 
Table S3: Assignment of transitions observed in the calculated UV/Vis spectra of compound 3ab

\begin{tabular}{|c|c|c|c|}
\hline $\begin{array}{l}\text { Wavelength } \\
\qquad(\mathrm{nm})\end{array}$ & $\begin{array}{l}\text { Oscillator } \\
\text { Strength } \\
\text { (f) }\end{array}$ & Assignment & $\begin{array}{l}\text { Excited } \\
\text { State }\end{array}$ \\
\hline 633.35 & 0.0039 & $\begin{array}{l}H-2 \rightarrow L(3.53 \%), H-2 \rightarrow L+5(5.50 \%), \\
H-1 \rightarrow L+4(18.36 \%), H \rightarrow L(27.77 \%) \\
H \rightarrow L+1(4.07 \%), H \rightarrow L+5 \text { (30.87\%) }\end{array}$ & 1 \\
\hline 404.83 & 0.0015 & $\begin{array}{l}H-4 \rightarrow L(16.62 \%), H-4 \rightarrow L+1(2.42 \%), \\
H-4 \rightarrow L+5(18.69 \%), H-2 \rightarrow L(4.49 \%), \\
H-2 \rightarrow L+5(3.54 \%), H-1 \rightarrow L+4 \\
(33.69 \%), H \rightarrow L(10.01 \%), H \rightarrow L+5 \\
(2.74 \%)\end{array}$ & 5 \\
\hline 383.53 & 0.0002 & $\begin{array}{l}H-4 \rightarrow L+4(53.85 \%), H-1 \rightarrow L \\
(12.20 \%), H-1 \rightarrow L+5(3.29 \%), H \rightarrow L+4 \\
(24.09 \%)\end{array}$ & 6 \\
\hline 335.05 & 0.0489 & $\begin{array}{l}\mathrm{H}-2 \rightarrow \mathrm{L}(3.77 \%), \mathrm{H}-1 \rightarrow \mathrm{L}(9.74 \%), \mathrm{H}- \\
1 \rightarrow \mathrm{L}+1(33.14 \%), \mathrm{H} \rightarrow \mathrm{L}(19.59 \%), \\
H \rightarrow L+5(19.55 \%)\end{array}$ & 7 \\
\hline 333.75 & 0.0771 & $\begin{array}{l}\mathrm{H}-2 \rightarrow \mathrm{L}(6.51 \%), \mathrm{H}-1 \rightarrow \mathrm{L}(8.42 \%), \mathrm{H}- \\
1 \rightarrow \mathrm{L}+1(37.49 \%), H \rightarrow \mathrm{L}(19.34 \%) \\
H \rightarrow L+1(15.68 \%), H \rightarrow L+5(2.26 \%)\end{array}$ & 8 \\
\hline 319.27 & 0.1698 & $\begin{array}{l}H-3 \rightarrow L(12.31 \%), H-2 \rightarrow L(21.92 \%), \\
H \rightarrow L(9.22 \%), H \rightarrow L+1(42.37 \%) \\
H \rightarrow L+5(5.29 \%)\end{array}$ & 9 \\
\hline 295.95 & 0.0637 & $\begin{array}{l}\mathrm{H}-3 \rightarrow \mathrm{L}(9.45 \%), \mathrm{H}-2 \rightarrow \mathrm{L}(51.10 \%), \mathrm{H}- \\
2 \rightarrow \mathrm{L}+1(4.33 \%), \mathrm{H}-1 \rightarrow \mathrm{L}(3.16 \%), \mathrm{H} \rightarrow \\
\mathrm{L}(4.88 \%), \mathrm{H} \rightarrow \mathrm{L}+5(15.93 \%)\end{array}$ & 10 \\
\hline 293.16 & 0.0526 & $\begin{array}{l}\mathrm{H}-2 \rightarrow \mathrm{L}(3.45 \%), \mathrm{H}-1 \rightarrow \mathrm{L}(28.82 \%), \mathrm{H}- \\
1 \rightarrow \mathrm{L}+2(13.87 \%), \mathrm{H}-1 \rightarrow \mathrm{L}+5 \\
(38.48 \%)\end{array}$ & 11 \\
\hline 288.12 & 0.3829 & $\begin{array}{l}H-4 \rightarrow L(3.71 \%), H-3 \rightarrow L(55.70 \%), H- \\
2 \rightarrow L+1(5.69 \%), H \rightarrow L(2.51 \%) \\
H \rightarrow L+1(7.23 \%), H \rightarrow L+2(3.77 \%) \\
H \rightarrow L+5(13.21 \%)\end{array}$ & 12 \\
\hline 281.92 & 0.8789 & $\begin{array}{l}\mathrm{H}-3 \rightarrow \mathrm{L}(10.63 \%), \mathrm{H}-2 \rightarrow \mathrm{L}+1 \\
(75.24 \%), \mathrm{H} \rightarrow \mathrm{L}+5(3.69 \%)\end{array}$ & 13 \\
\hline 273.94 & 0.0747 & $\begin{array}{l}H-3 \rightarrow L+2(8.70 \%), H-2 \rightarrow L+2 \\
(6.63 \%), H \rightarrow L+2(71.91 \%), H \rightarrow L+5 \\
(3.69 \%)\end{array}$ & 14 \\
\hline
\end{tabular}




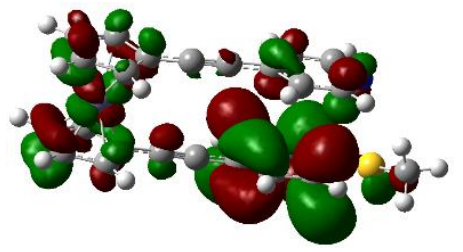

LUMO+5

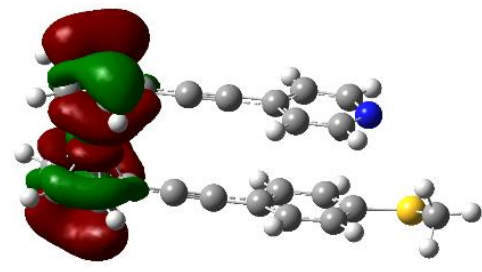

LUMO+2

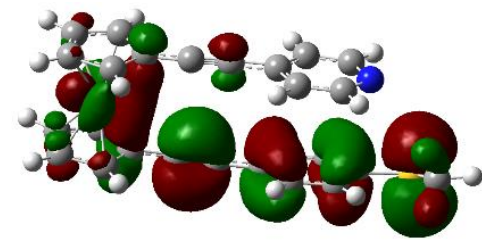

HOMO

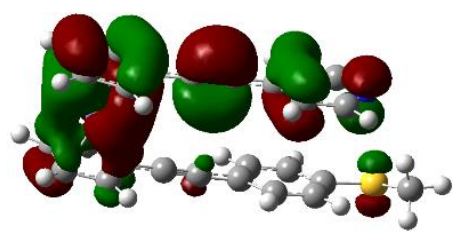

HOMO-3

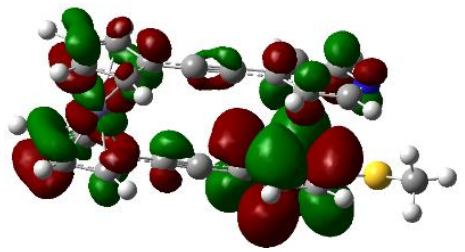

LUMO+4

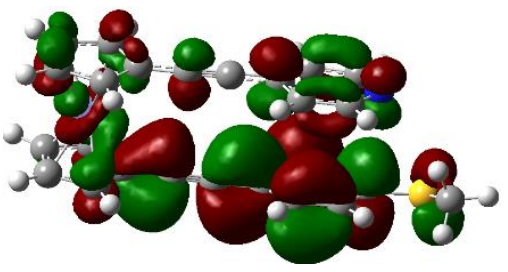

LUMO+1

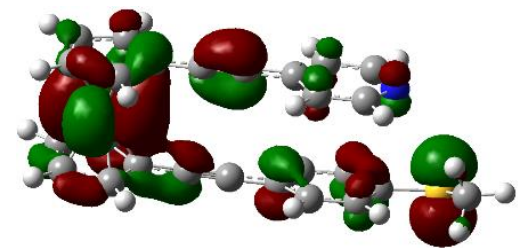

HOMO-1

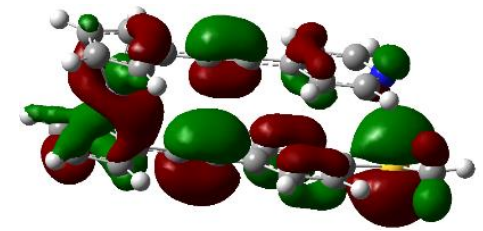

HOMO-4

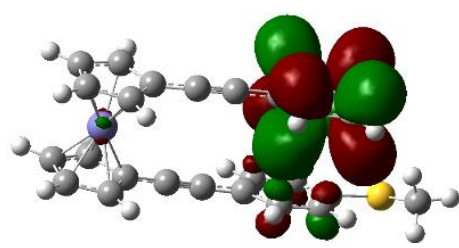

LUMO+3

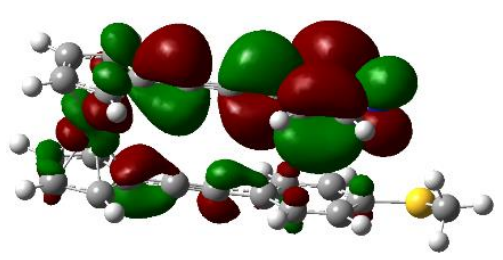

LUMO

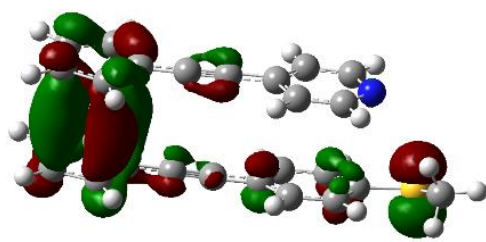

HOMO-2

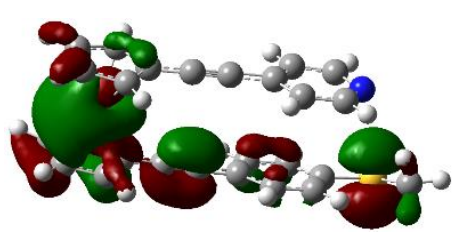

HOMO-5

Figure S88: Calculated iso-surfaces of HOMO-5 to LUMO-5 for compound 3ac

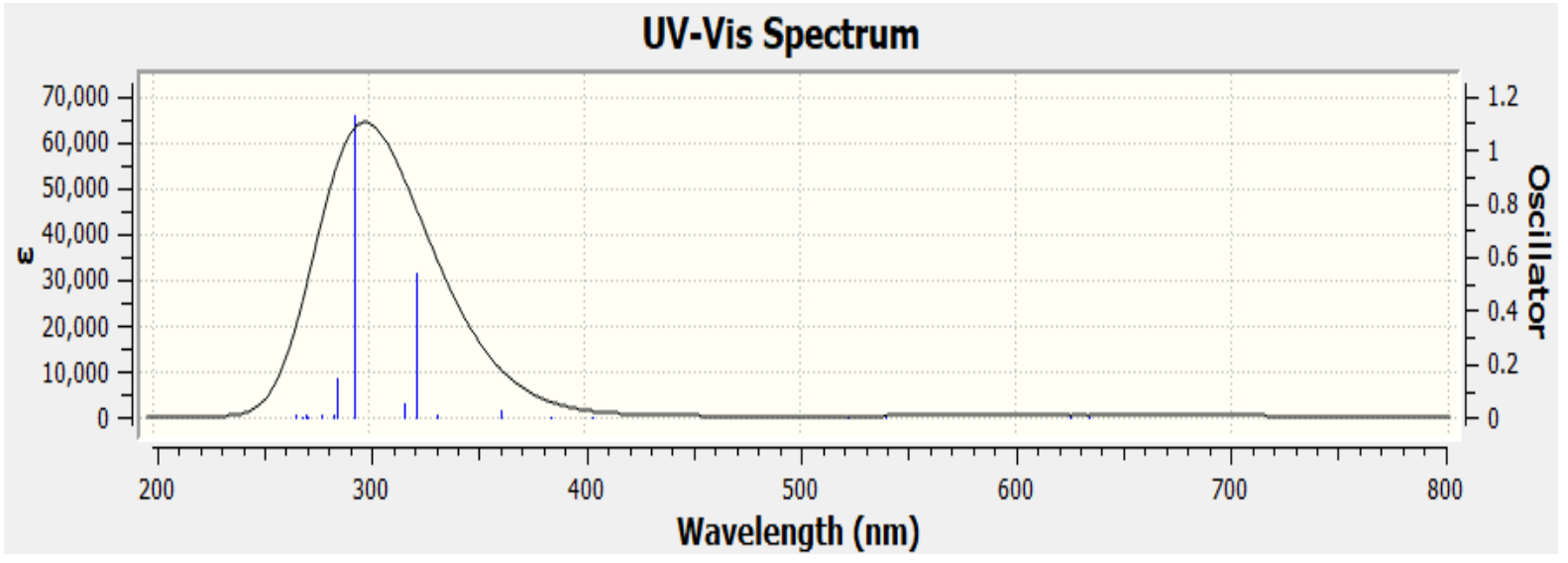

Figure S89: Calculated UV/Vis spectrum for compound 3ac 
Table S4: Assignment of transitions observed in the calculated UV/Vis spectra of compound 3ac

\begin{tabular}{|c|c|c|c|}
\hline $\begin{array}{l}\text { Wavelength } \\
\qquad(\mathrm{nm})\end{array}$ & $\begin{array}{l}\text { Oscillator } \\
\text { Strength } \\
\text { (f) }\end{array}$ & Assignment & $\begin{array}{c}\text { Excited } \\
\text { State }\end{array}$ \\
\hline 633.72 & 0.0036 & $\begin{array}{l}H-2 \rightarrow L(10.20 \%), H-2 \rightarrow L+1(3.14 \%), \\
H-2 \rightarrow L+3(5.47 \%), H-2 \rightarrow L+4 \\
(3.64 \%), H-2 \rightarrow L+5(12.18 \%), H-1 \rightarrow L \\
(5.35 \%), H-1 \rightarrow L+1(3.48 \%), H- \\
1 \rightarrow L+3(8.02 \%), H-1 \rightarrow L+5(9.39 \%) \\
H \rightarrow L(11.33 \%), H \rightarrow L+1(4.41 \%) \\
H \rightarrow L+5(11.71 \%)\end{array}$ & 1 \\
\hline 404.15 & 0.0019 & $\begin{array}{l}H-5 \rightarrow L(8.82 \%), H-5 \rightarrow L+1(2.92 \%), \\
H-5 \rightarrow L+5(10.30 \%), H-4 \rightarrow L(5.75 \%), \\
H-4 \rightarrow L+1(3.16 \%), H-4 \rightarrow L+5 \\
(7.73 \%), H-3 \rightarrow L(4.00 \%), H-3 \rightarrow L+5 \\
(3.22 \%), H-2 \rightarrow L(2.38 \%), H-2 \rightarrow L+3 \\
(11.12 \%), H-2 \rightarrow L+4(3.40 \%), H- \\
2 \rightarrow L+5(2.27 \%), H-1 \rightarrow L(3.82 \%), H- \\
1 \rightarrow L 3(13.31 \%), H-1 \rightarrow L+4(5.64 \%) . \\
H \rightarrow L(6.25 \%)\end{array}$ & 5 \\
\hline 361.85 & 0.0268 & $\begin{array}{l}H-2 \rightarrow L(5.46 \%), H-2 \rightarrow L+1(3.42 \%), \\
H-2 \rightarrow L+5(2.03 \%), H-1 \rightarrow L(2.81 \%), \\
H \rightarrow L(76.74 \%)\end{array}$ & 7 \\
\hline 322.55 & 0.5364 & $\begin{array}{l}H-3 \rightarrow L(11.35 \%), H-2 \rightarrow L(2.13 \%), H- \\
1 \rightarrow L(6.39 \%), H-1 \rightarrow L+1(2.73 \%) \\
H \rightarrow L+1(70.12 \%)\end{array}$ & 9 \\
\hline 294.04 & 1.1321 & $\begin{array}{l}\mathrm{H}-4 \rightarrow \mathrm{L}(2.72 \%), \mathrm{H}-3 \rightarrow \mathrm{L}(45.74 \%), \mathrm{H}- \\
2 \rightarrow \mathrm{L}+1(12.43 \%), \mathrm{H}-1 \rightarrow \mathrm{L}(3.88 \%), \mathrm{H}- \\
1 \rightarrow \mathrm{L}+1(16.13 \%), \mathrm{H} \rightarrow \mathrm{L}+1 \text { (9.23\%), }\end{array}$ & 11 \\
\hline 285.67 & 0.1491 & $\begin{array}{l}\mathrm{H}-5 \rightarrow \mathrm{L}(2.81 \%), \mathrm{H}-3 \rightarrow \mathrm{L}(24.02 \%), \mathrm{H}- \\
2 \rightarrow \mathrm{L}+1(9.99 \%), \mathrm{H}-2 \rightarrow \mathrm{L}+5(4.16 \%) \\
\mathrm{H}-1 \rightarrow \mathrm{L}+1(8.72 \%), \mathrm{H}-1 \rightarrow \mathrm{L}+5 \\
(5.40 \%), \mathrm{H} \rightarrow \mathrm{L}+1(3.09 \%), \mathrm{H} \rightarrow \mathrm{L}+5 \\
(28.19 \%)\end{array}$ & 12 \\
\hline
\end{tabular}




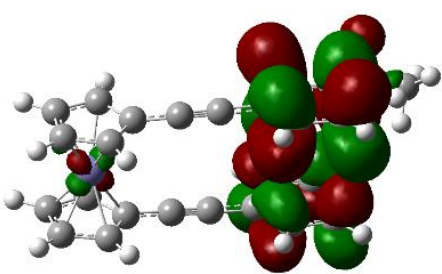

LUMO+5

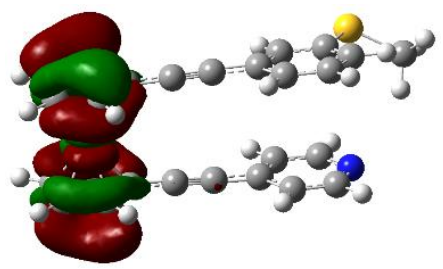

LUMO+2

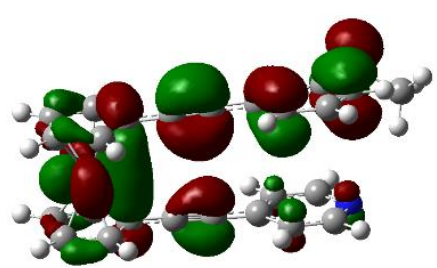

HOMO

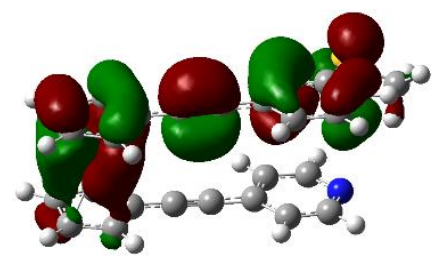

HOMO-3

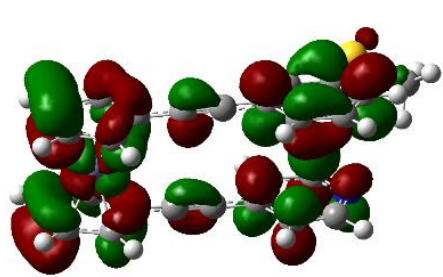

LUMO+4

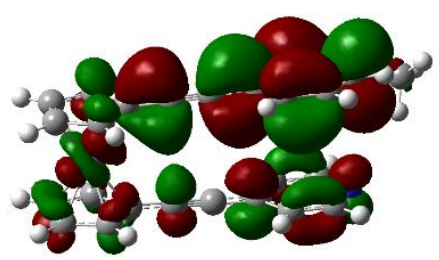

LUMO+1

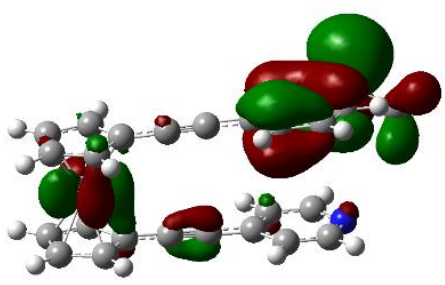

HOMO-1

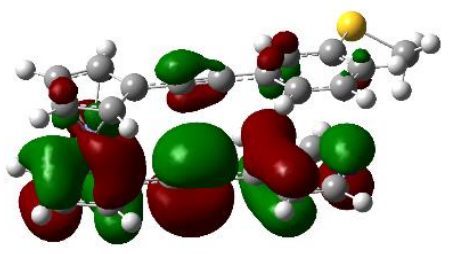

HOMO-4

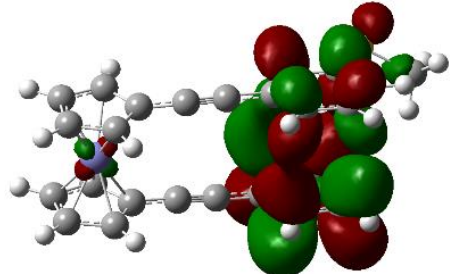

LUMO+3

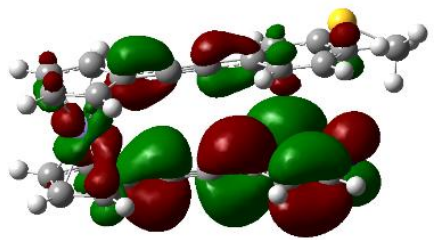

LUMO

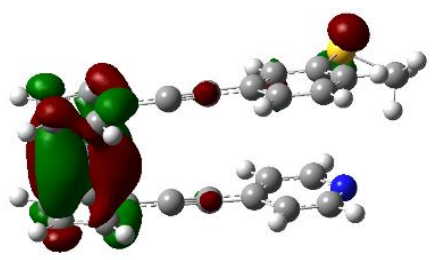

HOMO-2

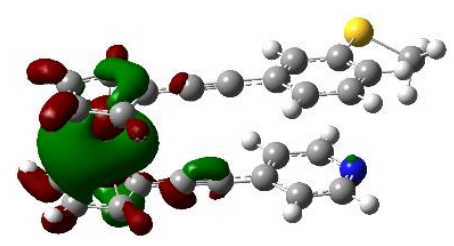

HOMO-5

Figure 590: Calculated iso-surfaces of HOMO-5 to LUMO-5 for compound 3ad

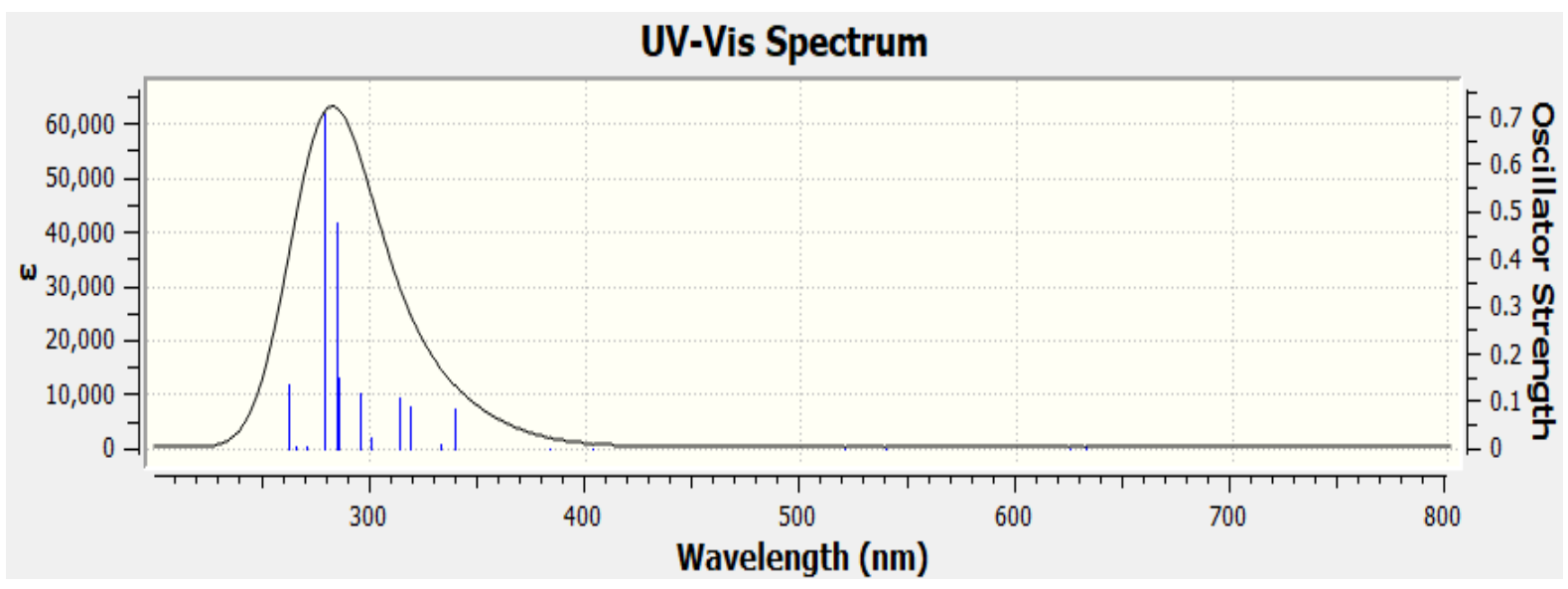

Figure S91: Calculated UV/Vis spectrum for compound 3ad 
Table S5: Assignment of transitions observed in the calculated UV/Vis spectra of compound 3ad

\begin{tabular}{|c|c|c|c|}
\hline $\begin{array}{c}\text { Wavelength } \\
(\mathrm{nm})\end{array}$ & $\begin{array}{l}\text { Oscillator } \\
\text { Strength } \\
\text { (f) }\end{array}$ & Assignment & $\begin{array}{l}\text { Excited } \\
\text { State }\end{array}$ \\
\hline 631.91 & 0.0033 & $\begin{array}{l}H-3 \rightarrow L(2.41 \%), H \rightarrow L+5(4.04 \%), H- \\
2 \rightarrow L(3.05 \%), H-2 \rightarrow L+5(4.06 \%), H- \\
1 \rightarrow L+3(15.00 \%), H-1 \rightarrow L+4(3.43 \%), \\
H \rightarrow L(22.22 \%), H \rightarrow L+1(8.44 \%), \\
H \rightarrow L+5(25.84 \%)\end{array}$ & 1 \\
\hline 403.70 & 0.0015 & $\begin{array}{l}H-5 \rightarrow L(14.72 \%), H-5 \rightarrow L+1(5.59 \%), \\
H-5 \rightarrow L+5(17.63 \%), H-4 \rightarrow L(2.41 \%), \\
H-3 \rightarrow L(2.84 \%), H-3 \rightarrow L+5(2.56 \%) \\
H-1 \rightarrow L+3(28.12 \%), H-1 \rightarrow L+4 \\
(6.11 \%), H \rightarrow L(8.63 \%), H \rightarrow L+5 \\
(2.20 \%)\end{array}$ & 5 \\
\hline 339.58 & 0.0839 & $\begin{array}{l}H-3 \rightarrow L(5.99 \%), H-3 \rightarrow L+3(2.89 \%), \\
H-2 \rightarrow L(5.55 \%), H-2 \rightarrow L+1(2.96 \%), \\
H \rightarrow L(55.30 \%), H \rightarrow L+1(16.57 \%), \\
H \rightarrow L+5(3.38 \%)\end{array}$ & 7 \\
\hline 318.95 & 0.0877 & $\mathrm{H}-2 \rightarrow \mathrm{L}(76.07 \%), \mathrm{H} \rightarrow \mathrm{L}+1$ (15.67\%) & 9 \\
\hline 314.26 & 0.1081 & $\begin{array}{l}H-4 \rightarrow L(18.70 \%), H-3 \rightarrow L(21.64 \%), \\
H-3 \rightarrow L+1(2.60 \%), H-2 \rightarrow L(8.74 \%), \\
H \rightarrow L(2.01 \%), H \rightarrow L+1(34.41 \%), \\
H \rightarrow L+5(3.36 \%)\end{array}$ & 10 \\
\hline 295.86 & 0.1162 & $\begin{array}{l}H-4 \rightarrow L(17.86 \%), H-3 \rightarrow L(40.63 \%) \\
H-3 \rightarrow L+1(9.11 \%), H-2 \rightarrow L+1 \\
(13.83 \%), H \rightarrow L(2.50 \%) H \rightarrow L+5 \\
(6.65 \%)\end{array}$ & 12 \\
\hline 285.66 & 0.1504 & $\begin{array}{l}H-4 \rightarrow L(4.09 \%), H-1 \rightarrow L(18.28 \%), H- \\
1 \rightarrow L+1(20.74 \%), H-1 \rightarrow L+5 \\
(37.26 \%), H \rightarrow L+1(2.47 \%)\end{array}$ & 13 \\
\hline 284.71 & 0.4782 & $\begin{array}{l}\mathrm{H}-5 \rightarrow \mathrm{L}(2.55 \%), \mathrm{H}-4 \rightarrow \mathrm{L}(41.70 \%), \mathrm{H}- \\
1 \rightarrow \mathrm{L}+1(2.45 \%), \mathrm{H}-1 \rightarrow \mathrm{L}+5(5.16 \%) \\
\mathrm{H} \rightarrow \mathrm{L}(2.04 \%), \mathrm{H} \rightarrow \mathrm{L}+1(12.37 \%) \\
\mathrm{H} \rightarrow \mathrm{L}+5(21.39 \%)\end{array}$ & 14 \\
\hline 279.54 & 0.7065 & $\begin{array}{l}\mathrm{H}-4 \rightarrow \mathrm{L}(5.59 \%), \mathrm{H}-3 \rightarrow \mathrm{L}+1(69.37 \%), \\
\mathrm{H}-2 \rightarrow \mathrm{L}+1(3.19 \%), \mathrm{H} \rightarrow \mathrm{L}+5(8.77 \%)\end{array}$ & 15 \\
\hline 262.88 & 0.1369 & $\begin{array}{l}H-7 \rightarrow L(5.53 \%), H-3 \rightarrow L+1(2.08 \%) \\
H-2 \rightarrow L+1(2.30 \%), H-2 \rightarrow L+2 \\
(11.55 \%), H-2 \rightarrow L+4(3.18 \%), H \rightarrow L+2 \\
(63.93 \%)\end{array}$ & 18 \\
\hline
\end{tabular}




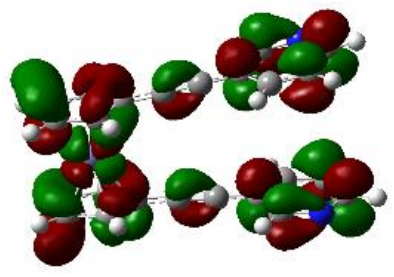

LUMO+5

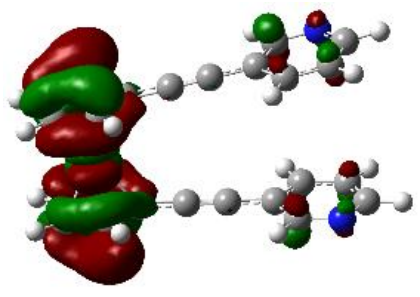

$\mathrm{LUMO}+4$

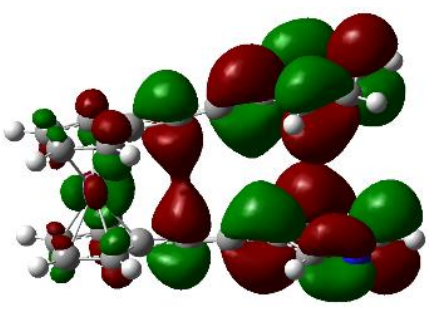

LUMO+1

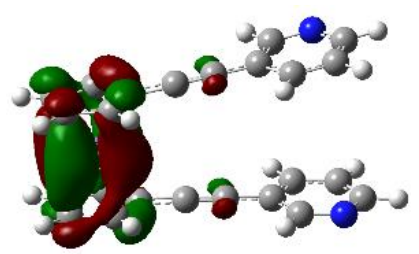

HOMO-1

HOMO-4

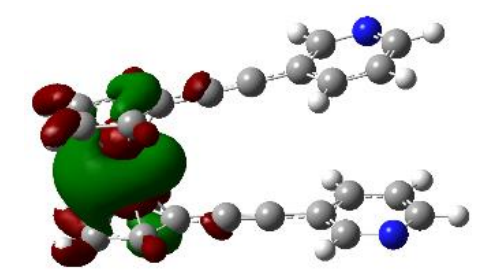

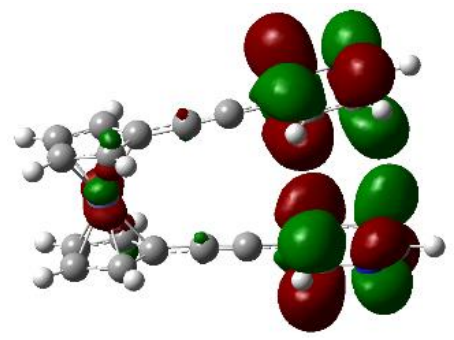

LUMO+3

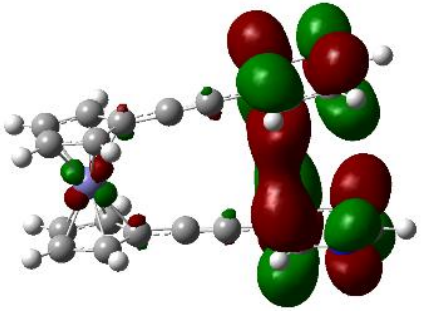

LUMO+2

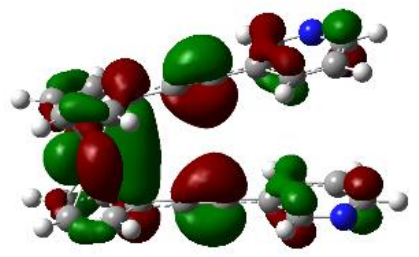

HOMO

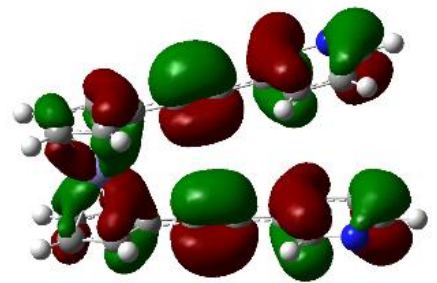

HOMO-3

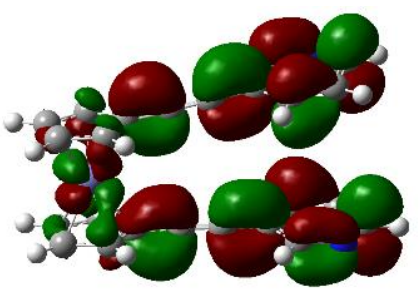

LUMO

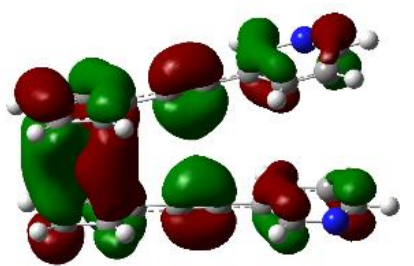

HOMO-2

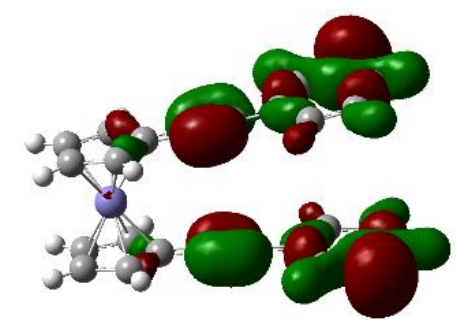

HOMO-5

Figure S92: Calculated iso-surfaces of HOMO-5 to LUMO-5 for compound $3 \mathbf{b b}$

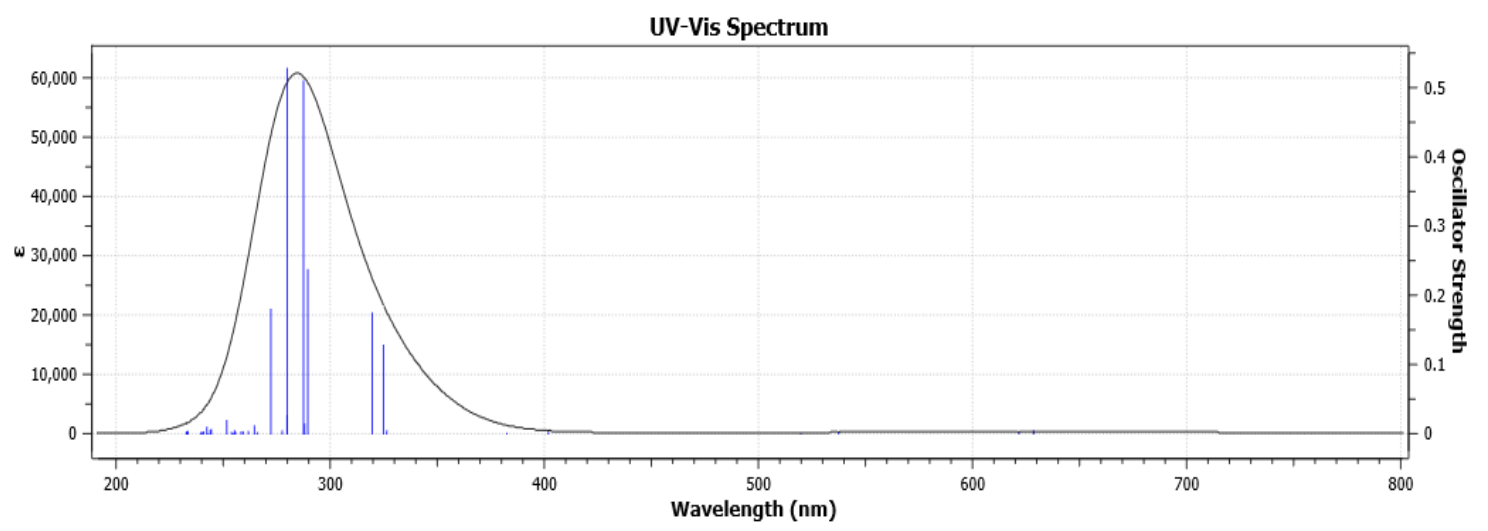

Figure S93: Calculated UV/Vis spectrum for compound $\mathbf{3 b b}$ 
Table S6: Assignment of transitions observed in the calculated UV/Vis spectra of compound $\mathbf{3 b b}$

\begin{tabular}{|c|c|c|c|}
\hline $\begin{array}{l}\text { Wavelength } \\
\qquad(\mathrm{nm})\end{array}$ & $\begin{array}{l}\text { Oscillator } \\
\text { Strength } \\
\text { (f) }\end{array}$ & Assignment & $\begin{array}{l}\text { Excited } \\
\text { State }\end{array}$ \\
\hline 628.53 & 0.0038 & $\begin{array}{l}H-2 \rightarrow L(5.10 \%), H-2 \rightarrow L+6(6.60 \%), \\
H-1 \rightarrow L+5(17.41 \%), H \rightarrow L(30.76 \%), \\
H \rightarrow L+2(2.12 \%), H \rightarrow L+5(29.15)\end{array}$ & 1 \\
\hline 401.88 & 0.0012 & $\begin{array}{l}H-4 \rightarrow L(19.56 \%), H-4 \rightarrow L+5 \\
(18.89 \%), H-3 \rightarrow L(6.91 \%), H-3 \rightarrow L+5 \\
(4.64 \%), H-1 \rightarrow L+4(34.64 \%), H \rightarrow \\
L+4, H \rightarrow L(9.61 \%), H \rightarrow L+5(2.83 \%)\end{array}$ & 5 \\
\hline 382.55 & 0.0001 & $\begin{array}{l}\mathrm{H}-5 \rightarrow \mathrm{L}+4(55 \%), \mathrm{H}-1 \rightarrow \mathrm{L}(12.12 \%), \mathrm{H}- \\
1 \rightarrow \mathrm{L}+5(4.01 \%), \mathrm{H} \rightarrow \mathrm{L}+4(25.34 \%)\end{array}$ & 6 \\
\hline 326.41 & 0.032 & $\begin{array}{l}\mathrm{H}-1 \rightarrow \mathrm{L}+1(89.67), \mathrm{H}-1 \rightarrow \mathrm{L}+11 \\
(5.11 \%)\end{array}$ & 7 \\
\hline 324.89 & 0.1274 & $\mathrm{H}-3 \rightarrow \mathrm{L}(7.40 \%), \mathrm{H} \rightarrow \mathrm{L}+1$ (85.23\%) & 8 \\
\hline 319.63 & 0.1740 & $\begin{array}{l}H-3 \rightarrow L+1(9.10 \%), H-2 \rightarrow L(35.63 \%) \\
H-2 \rightarrow L+5(2.83 \%), H \rightarrow L(44.40 \%) \\
H \rightarrow L+5(9.05 \%)\end{array}$ & 9 \\
\hline 289.55 & 0.2364 & $\begin{array}{l}H-3 \rightarrow L(16.62 \%), H-1 \rightarrow L(37.90 \%), \\
H-1 \rightarrow L+2(3.53 \%), H-1 \rightarrow L+5 \\
(30.49 \%), H \rightarrow L+1(2.28 \%)\end{array}$ & 10 \\
\hline 287.55 & 0.5088 & $\begin{array}{l}\mathrm{H}-3 \rightarrow \mathrm{L}(64.83 \%), \mathrm{H}-2 \rightarrow \mathrm{L}+1(3.03 \%) \\
\mathrm{H}-1 \rightarrow \mathrm{L}(7.99 \%), \mathrm{H}-1 \rightarrow \mathrm{L}+5(9.95 \%) \\
\mathrm{H} \rightarrow \mathrm{L}+1 \text { (3.99\%) }\end{array}$ & 12 \\
\hline 279.93 & 0.5277 & $\begin{array}{l}H-2 \rightarrow L+1(81.52 \%), H \rightarrow L+1(2.37 \%) \\
H \rightarrow L+3(7.27 \%)\end{array}$ & 13 \\
\hline 272.30 & 0.1793 & $\begin{array}{l}H-3 \rightarrow L(2.10 \%), H-3 \rightarrow L+2(3.66 \%) \\
H-2 \rightarrow L+2(6.48 \%), H-2 \rightarrow L+3 \\
(3.91 \%), H \rightarrow L+3(79.31 \%)\end{array}$ & 16 \\
\hline
\end{tabular}



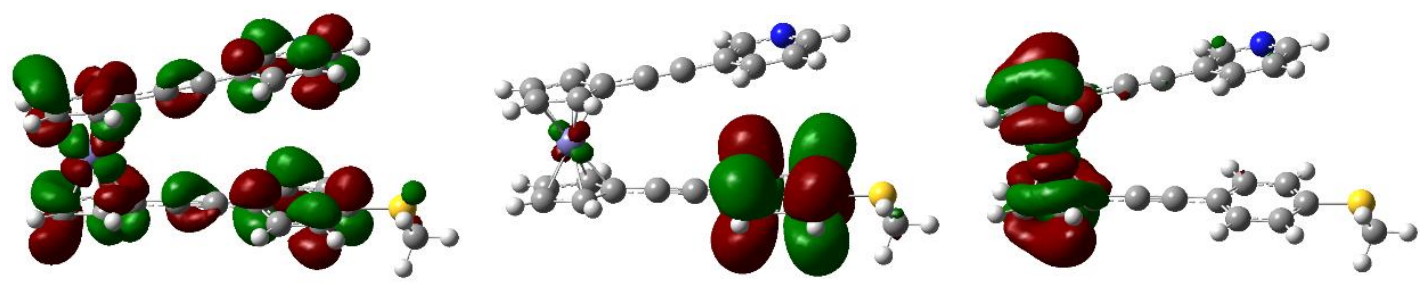

LUMO+5

LUMO+4

LUMO+3
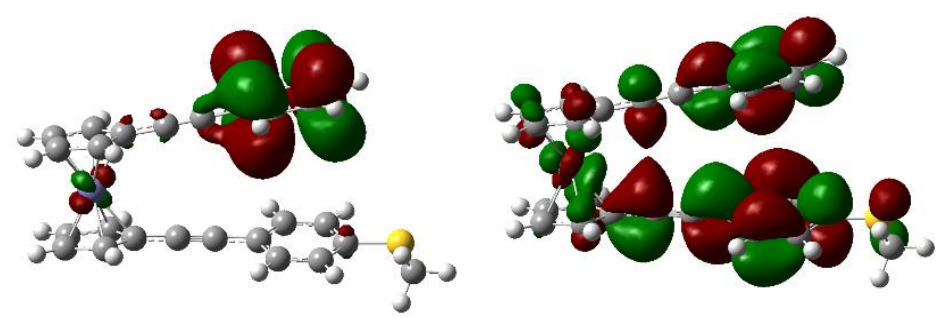

LUMO+2

LUMO+1

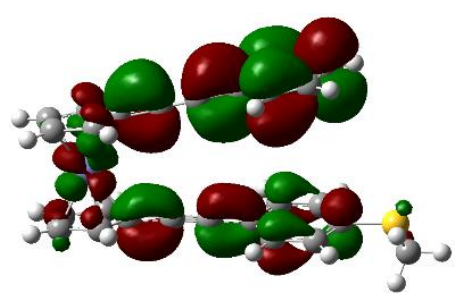

LUMO

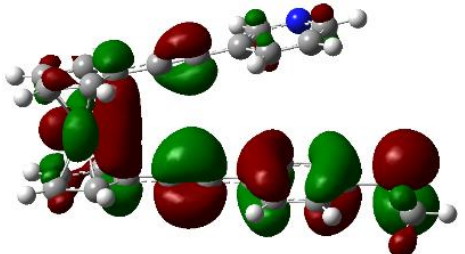

HOMO

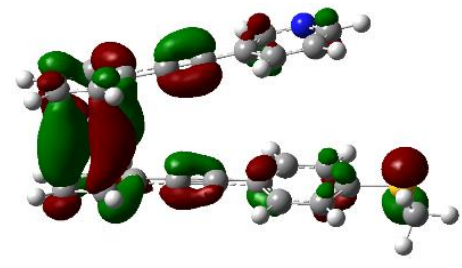

HOMO-1

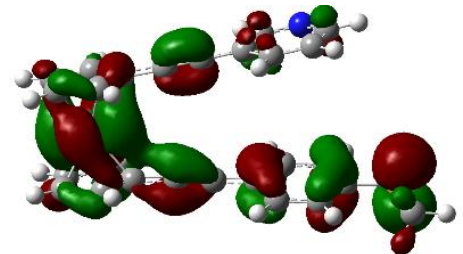

HOMO-2

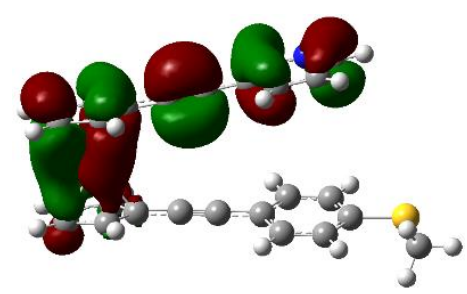

HOMO-3

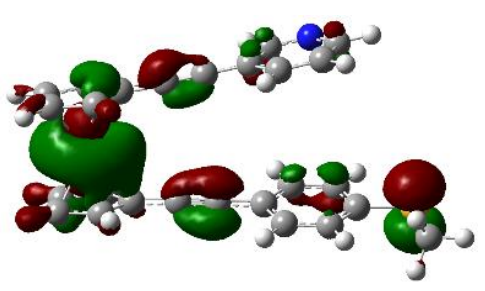

HOMO-4

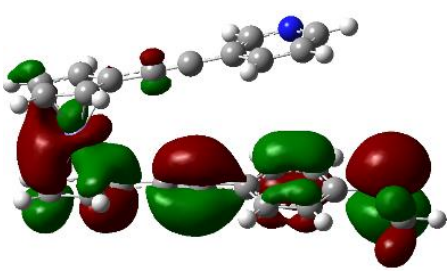

HOMO-5

Figure S94: Calculated iso-surfaces of HOMO-5 to LUMO-5 for compound 3bc

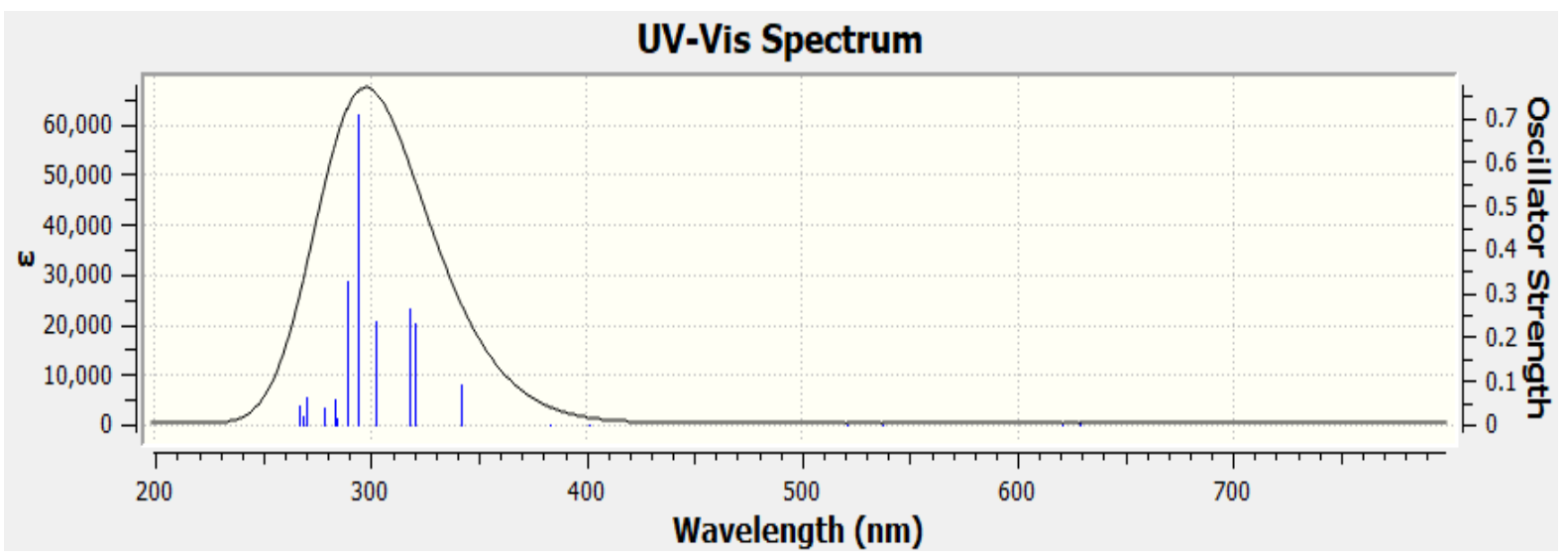

Figure S95: Calculated UV/Vis spectrum for compound 3bc 
Table S7: Assignment of transitions observed in the calculated UV/Vis spectra of compound 3bc

\begin{tabular}{|c|c|c|c|}
\hline $\begin{array}{l}\text { Wavelength } \\
\text { (nm) }\end{array}$ & $\begin{array}{l}\text { Oscillator } \\
\text { Strength } \\
\text { (f) }\end{array}$ & Assignment & Excited State \\
\hline 629.10 & 0.0042 & $\begin{array}{l}H-3 \rightarrow L(2.36 \%), H-3 \rightarrow L+5(3.46 \%), H-2 \rightarrow L \\
(10.72 \%), H-2 \rightarrow L+4(6.57 \%), H-3 \rightarrow L+5 \\
(11.43 \%), H-1 \rightarrow L(4.26 \%), H-1 \rightarrow L+4 \\
(12.25 \%), H-1 \rightarrow L+5(6.04 \%), H \rightarrow L(15.02 \%), \\
H \rightarrow L+1(2.24 \%) H \rightarrow L+5(13.78 \%)\end{array}$ & 1 \\
\hline 401.24 & 0.0015 & $\begin{array}{l}\mathrm{H}-5 \rightarrow \mathrm{L}(5.11 \%), \mathrm{H}-5 \rightarrow \mathrm{L}+5(5.12 \%), \mathrm{H}-4 \rightarrow \mathrm{L} \\
(13.26 \%), \mathrm{H}-4 \rightarrow \mathrm{L}+1(2.45 \%) \mathrm{H}-4 \rightarrow \mathrm{L}+5 \\
(14.07 \%), \mathrm{H}-3 \rightarrow \mathrm{L}(4.31 \%), \mathrm{H}-3 \rightarrow \mathrm{L}+5(2.93 \%), \\
\mathrm{H}-2 \rightarrow \mathrm{L}(3.45 \%), \mathrm{H}-2 \rightarrow \mathrm{L}+4(10.64 \%), \%), \mathrm{H}- \\
2 \rightarrow \mathrm{L}+5(2.42 \%), \mathrm{H}-1 \rightarrow \mathrm{L}(2.23 \%), \mathrm{H}-1 \rightarrow \mathrm{L}+4 \\
(21.58 \%), \mathrm{H} \rightarrow \mathrm{L}(5.12 \%)\end{array}$ & 5 \\
\hline 342.59 & 0.0925 & $\begin{array}{l}H-3 \rightarrow L(2.40 \%), H-2 \rightarrow L(7.35 \%), H-2 \rightarrow L+1 \\
(3.70 \%), H-1 \rightarrow L+5(2.04 \%), H-1 \rightarrow L(2.92 \%) \\
H \rightarrow L(72.22 \%), H \rightarrow L+1(2.10 \%)\end{array}$ & 7 \\
\hline 321.24 & 0.2327 & $\begin{array}{l}H-3 \rightarrow \mathrm{L}(6.79 \%), \mathrm{H}-2 \rightarrow \mathrm{L}(4.06 \%), \mathrm{H}-2 \rightarrow \mathrm{L}+1 \\
(11.83 \%), \mathrm{H}-1 \rightarrow \mathrm{L}(5.70 \%), \mathrm{H}-1 \rightarrow \mathrm{L}+1 \\
(21.64 \%), \mathrm{H} \rightarrow \mathrm{L}+1(39.80 \%)\end{array}$ & 8 \\
\hline 318.70 & 0.2663 & $\begin{array}{l}H-3 \rightarrow L(6.66 \%), H-2 \rightarrow L+1(7.51 \%), H-1 \rightarrow L \\
(6.45 \%), H-1 \rightarrow L+1(30.05 \%), H-1 \rightarrow L+12 \\
(2.57 \%), H \rightarrow L+1(37.86 \%)\end{array}$ & 9 \\
\hline 303.12 & 0.2373 & $\begin{array}{l}\mathrm{H}-3 \rightarrow \mathrm{L}(6.01 \%), \mathrm{H}-2 \rightarrow \mathrm{L}(36.76 \%), \mathrm{H}-2 \rightarrow \mathrm{L}+2 \\
(19.38 \%), \mathrm{H}-2 \rightarrow \mathrm{L}+5(2.81 \%), \mathrm{H}-1 \rightarrow \mathrm{L} \\
(17.55 \%), \mathrm{H}-1 \rightarrow \mathrm{L}+1(7.16 \%), \mathrm{H} \rightarrow \mathrm{L}+5(2.88 \%)\end{array}$ & 10 \\
\hline 294.51 & 0.7088 & $\begin{array}{l}H-3 \rightarrow L(19.35 \%), H-2 \rightarrow L+2(24.38 \%), H-1 \rightarrow L \\
(4.75 \%), H-1 \rightarrow L+1(14.28 \%), H \rightarrow L+1(9.07 \%), \\
H \rightarrow L+2(16.94 \%)\end{array}$ & 11 \\
\hline 289.59 & 0.3271 & $\begin{array}{l}H-3 \rightarrow L(19.70 \%), H \rightarrow L+1(2.17 \%), H \rightarrow L+2 \\
(69.17 \%)\end{array}$ & 12 \\
\hline 283.41 & 0.0594 & $\begin{array}{l}H-3 \rightarrow L(22.16 \%), H-2 \rightarrow L(2.84 \%), H-2 \rightarrow L+2 \\
(8.46 \%), H-2 \rightarrow L+5(4.65 \%), H-1 \rightarrow L+2 \\
(4.16 \%), H-1 \rightarrow L+5(3.71 \%), H \rightarrow L(2.36 \%) \\
H \rightarrow L+2(9.82 \%), H \rightarrow L+3(3.09 \%) H \rightarrow L+5 \\
(26.84 \%)\end{array}$ & 14 \\
\hline 270.20 & 0.0605 & $\begin{array}{l}H-4 \rightarrow \mathrm{L}(3.98 \%), H-4 \rightarrow \mathrm{L}+1(2.41 \%), H-3 \rightarrow \mathrm{L}+1 \\
(74.13 \%), H-2 \rightarrow \mathrm{L}+2(4.77 \%), H-1 \rightarrow L+2 \\
(4.08 \%)\end{array}$ & 16 \\
\hline
\end{tabular}




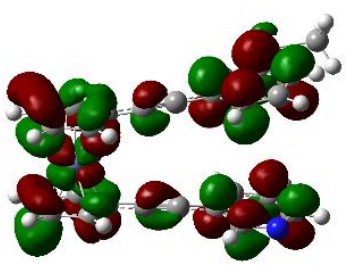

LUMO+5

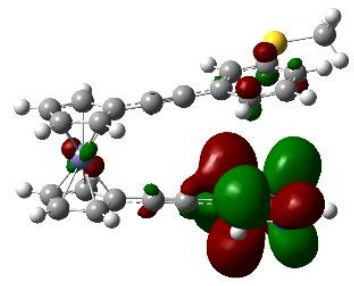

LUMO+2

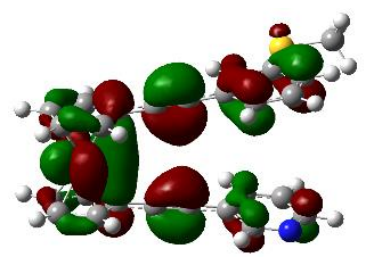

HOMO

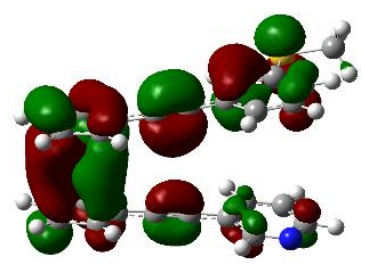

HOMO-3

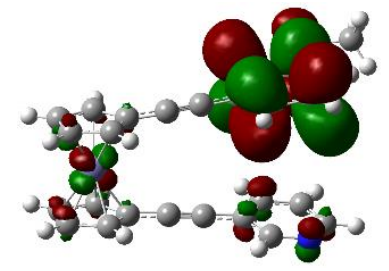

LUMO+4

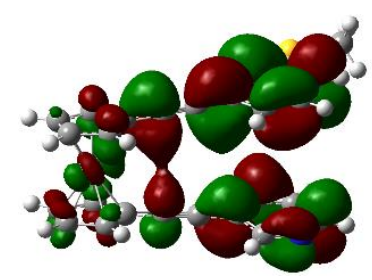

LUMO+1

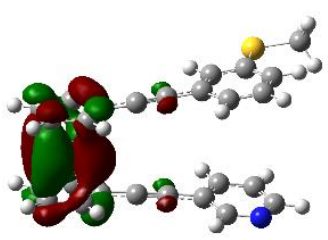

HOMO-1

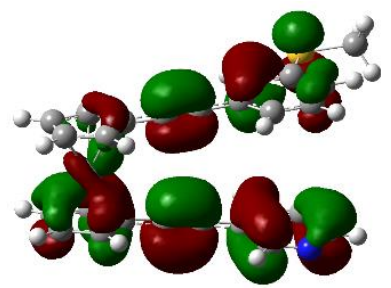

HOMO-4

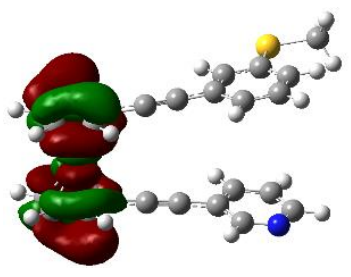

LUMO+3

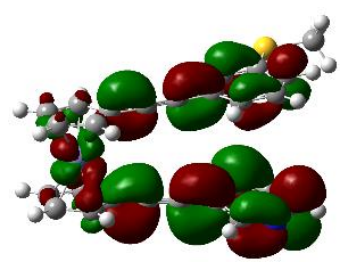

LUMO

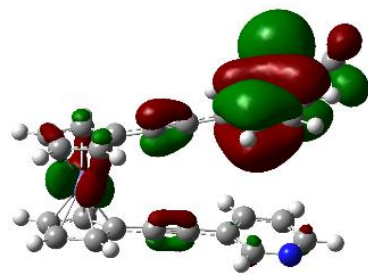

HOMO-2

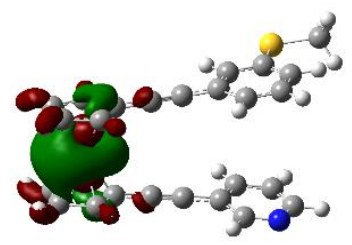

HOMO-5

Figure S96: Calculated iso-surfaces of HOMO-5 to LUMO-5 for compound 3bd

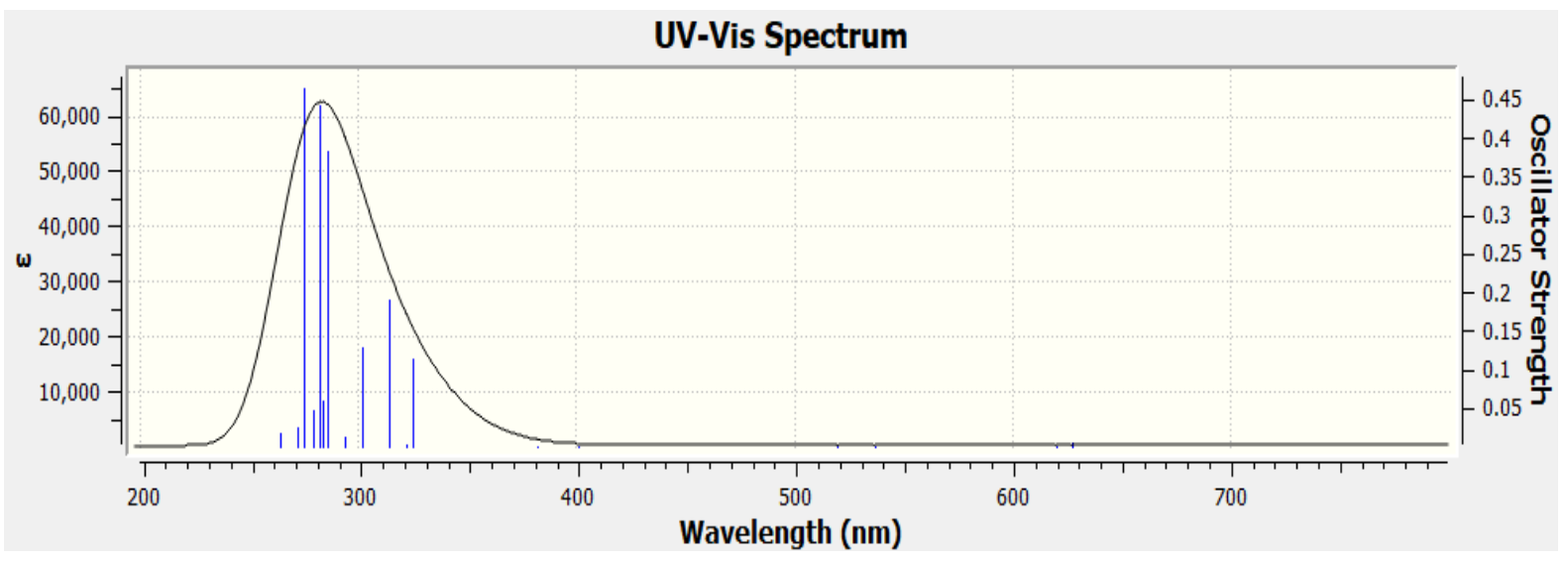

Figure S97: Calculated UV/Vis spectrum for compound 3 bd 
Table S8: Assignment of transitions observed in the calculated UV/Vis spectra of compound 3bd

\begin{tabular}{|c|c|c|c|}
\hline $\begin{array}{l}\text { Wavelength } \\
\qquad(\mathrm{nm})\end{array}$ & $\begin{array}{l}\text { Oscillator } \\
\text { Strength } \\
\text { (f) }\end{array}$ & Assignment & $\begin{array}{l}\text { Excited } \\
\text { State }\end{array}$ \\
\hline 627.37 & 0.0039 & $\begin{array}{l}H-3 \rightarrow L(4.35 \%), H-3 \rightarrow L+5(5.74 \%) H- \\
2 \rightarrow L(2.20 \%) H-2 \rightarrow L+5(2.53 \%) H- \\
1 \rightarrow L+3(2.67 \%) H-1 \rightarrow L+4(16.88 \%) \\
H \rightarrow L(26.71 \%) H \rightarrow L+1(3.99 \%) \\
H \rightarrow L+5(25.51 \%)\end{array}$ & 1 \\
\hline 400.91 & 0.0012 & $\begin{array}{l}H-5 \rightarrow L(18.51 \%), H-5 \rightarrow L+1(2.77 \%), \\
H-5 \rightarrow L+5(18.20 \%), H-3 \rightarrow L(5.53 \%), \\
H-3 \rightarrow L+5(3.87 \%), H-1 \rightarrow L 3(4.86 \%), \\
H-1 \rightarrow L 4(29.20 \%), H \rightarrow L(8.22 \%), \\
H \rightarrow L+5(2.46 \%)\end{array}$ & 5 \\
\hline 325.49 & 0.1126 & $\begin{array}{l}\mathrm{H}-3 \rightarrow \mathrm{L}(7.31 \%), \mathrm{H}-2 \rightarrow \mathrm{L}(5.68 \%), \mathrm{H}- \\
2 \rightarrow \mathrm{L}+1(2.83 \%), \mathrm{H} \rightarrow \mathrm{L}(41.17 \%) \\
\mathrm{H} \rightarrow \mathrm{L}+1(30.93 \%), \mathrm{H} \rightarrow \mathrm{L}+5(2.61 \%)\end{array}$ & 7 \\
\hline 314.7 & 0.1907 & $\begin{array}{l}H-4 \rightarrow L(11.39 \%), H-3 \rightarrow L(16.21 \%), \\
H-2 \rightarrow L(3.73 \%), H \rightarrow L(9.59 \%), \\
H \rightarrow L+1(45.88 \%), H \rightarrow L+5(3.63 \%)\end{array}$ & 9 \\
\hline 302.36 & 0.1284 & $\begin{array}{l}H-3 \rightarrow L(8.48 \%), H-3 \rightarrow L+1(2.83 \%), \\
H-2 \rightarrow L(68.38 \%), H-2 \rightarrow L+1(6.39 \%)\end{array}$ & 10 \\
\hline 286.04 & 0.3826 & $\begin{array}{l}H-4 \rightarrow L(34.07 \%), H-3 \rightarrow L(28.51 \%), \\
H-2 \rightarrow L+2(3.78 \%), H-1 \rightarrow L(2.05 \%) \\
H \rightarrow L(3.02 \%), H \rightarrow L+1(2.33 \%) \\
H \rightarrow L+2(8.64 \%), H \rightarrow L+5(6.91 \%)\end{array}$ & 12 \\
\hline 283.74 & 0.0601 & $\begin{array}{l}H-3 \rightarrow L(6.25 \%), H-1 \rightarrow L(27.69 \%), H- \\
1 \rightarrow L+1(5.27 \%), H-1 \rightarrow L+2(2.2 \%), H- \\
1 \rightarrow L+5(33.82 \%) H \rightarrow L+5(6.38 \%)\end{array}$ & 13 \\
\hline 282.54 & 0.4429 & $\begin{array}{l}H-4 \rightarrow L(38.82 \%), H-3 \rightarrow L(6.48 \%), H- \\
1 \rightarrow L(5.25 \%), H-1 \rightarrow L+5(8.04 \%) \\
H \rightarrow L(2.80 \%), H \rightarrow L+1(6.03 \%) \\
H \rightarrow L+2(2.51 \%), H \rightarrow L+5(18.11 \%)\end{array}$ & 14 \\
\hline 279.53 & 0.0462 & $\begin{array}{l}H-4 \rightarrow L+2(2.09 \%), H-3 \rightarrow L(5.85 \%), \\
H-3 \rightarrow L+1(18.27 \%), H \rightarrow L+2 \\
(61.07 \%), H \rightarrow L+5(3.27 \%)\end{array}$ & 15 \\
\hline 275.31 & 0.4639 & $\begin{array}{l}H-4 \rightarrow L+2(7.73 \%), H-3 \rightarrow L+2 \\
(55.59 \%), H \rightarrow L+2(15.85 \%), H \rightarrow L+5 \\
(5.2 \%)\end{array}$ & 16 \\
\hline
\end{tabular}




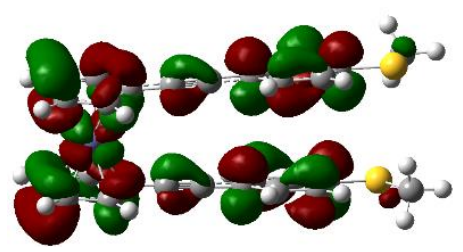

LUMO+5

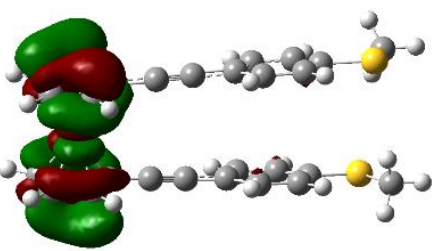

LUMO+2

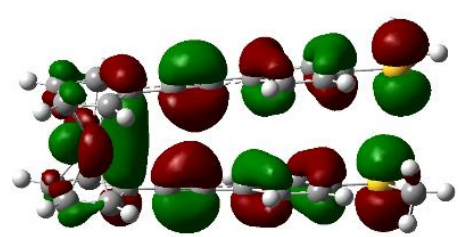

HOMO

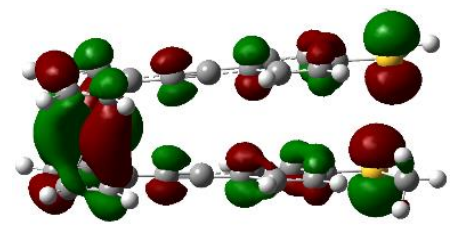

HOMO-3

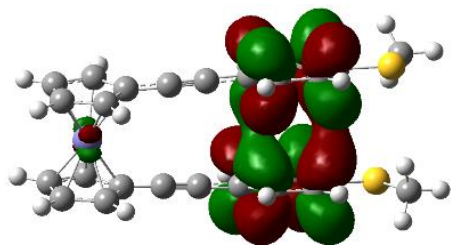

LUMO+4

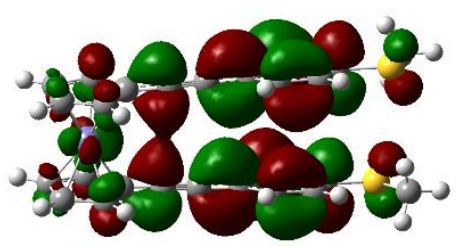

LUMO+1

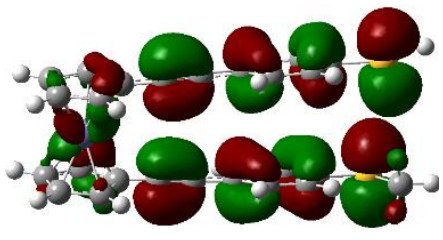

HOMO-1

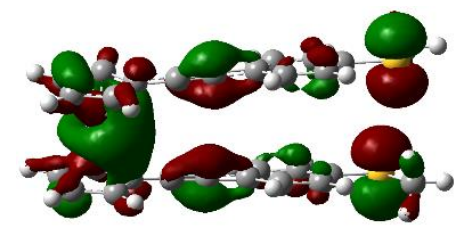

HOMO-4

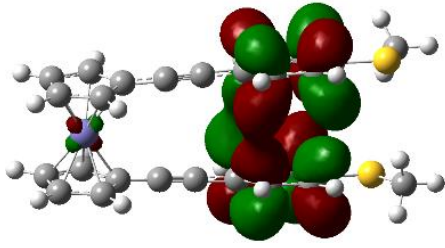

LUMO+3

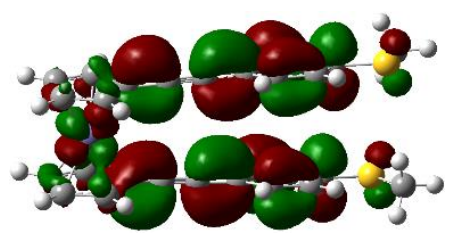

LUMO

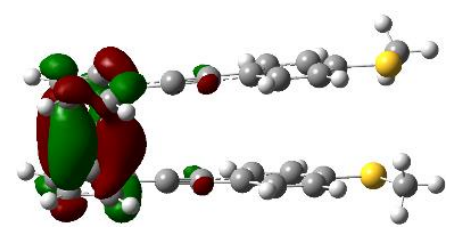

HOMO-2

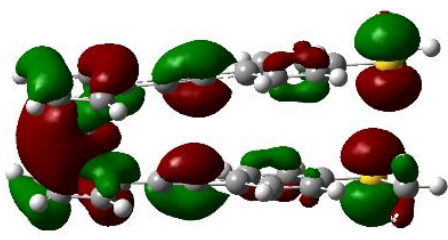

HOMO-5

Figure S98: Calculated iso-surfaces of HOMO-5 to LUMO-5 for compound 3cc

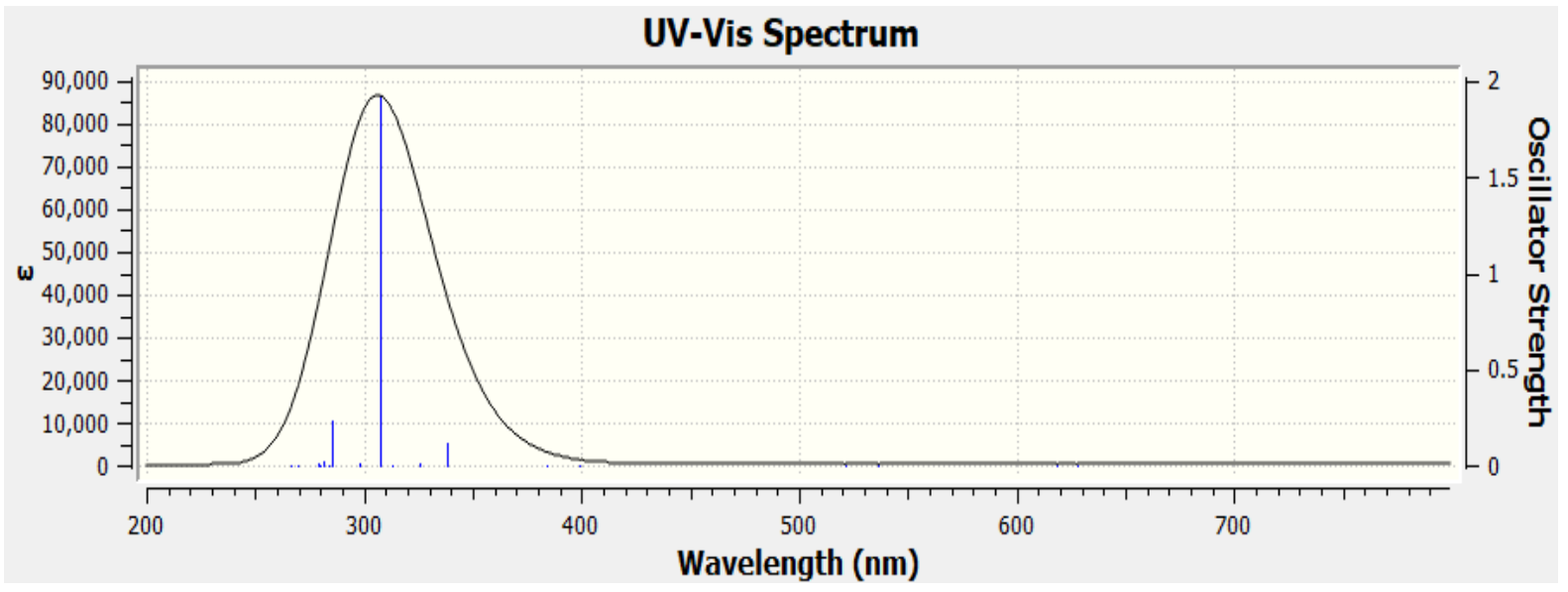

Figure S99: Calculated UV/Vis spectrum for compound 3cc 
Table S9: Assignment of transitions observed in the calculated UV/Vis spectra of compound 3cc

\begin{tabular}{|c|c|c|c|}
\hline $\begin{array}{c}\text { Wavelength } \\
(\mathrm{nm})\end{array}$ & $\begin{array}{l}\text { Oscillator } \\
\text { Strength } \\
\text { (f) }\end{array}$ & Assignment & $\begin{array}{c}\text { Excited } \\
\text { State }\end{array}$ \\
\hline 627.67 & 0.0036 & $\begin{array}{l}H-3 \rightarrow L(20.45 \%), H-3 \rightarrow L+5 \\
(19.98 \%), H-2 \rightarrow L+3(3.02 \%), H- \\
2 \rightarrow L+4(16.65 \%), H \rightarrow L(18.91 \%) \\
H \rightarrow L+5(13.76 \%)\end{array}$ & 1 \\
\hline 399.32 & 0.0013 & $\begin{array}{l}H-5 \rightarrow L(14.59 \%), H-5 \rightarrow L+5 \\
(12.59 \%), H-4 \rightarrow L(9.66 \%), H- \\
4 \rightarrow L+5(7.81 \%), H-3 \rightarrow L(9.59 \%) \\
H-3 \rightarrow L+5(4.87 \%), H-2 \rightarrow L+3 \\
(5.10 \%), H-2 \rightarrow L+4(27.76 \%) \\
H \rightarrow L(4.94 \%)\end{array}$ & 5 \\
\hline 388.56 & 0.1142 & $\begin{array}{l}H-3 \rightarrow L(19.37 \%), H-3 \rightarrow L+5 \\
(3.14 \%), H-1 \rightarrow L+1(2.88 \%), H \rightarrow L \\
(67.00 \%), H \rightarrow L+5(2.96 \%)\end{array}$ & 7 \\
\hline 307.44 & 1.9244 & $\begin{array}{l}H-1 \rightarrow L(57.92 \%), H \rightarrow L+1 \\
(36.40 \%)\end{array}$ & 10 \\
\hline 285.48 & 0.2295 & $\begin{array}{l}H-3 \rightarrow L+1(73.32 \%), H \rightarrow L+1 \\
(4.23 \%)\end{array}$ & 12 \\
\hline
\end{tabular}




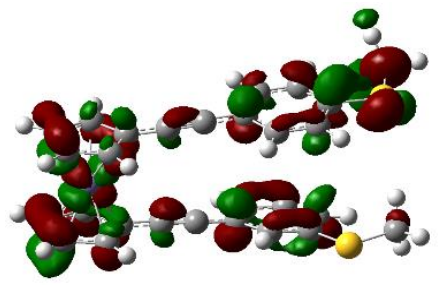

LUMO+5

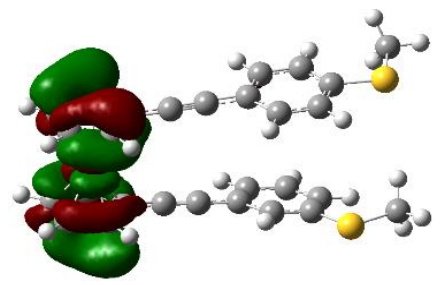

$\mathrm{LUMO}+2$

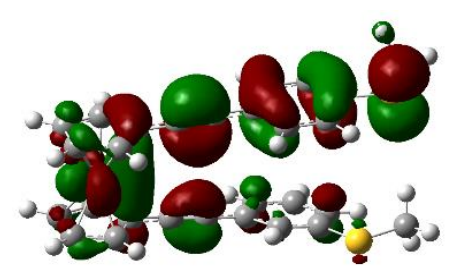

HOMO

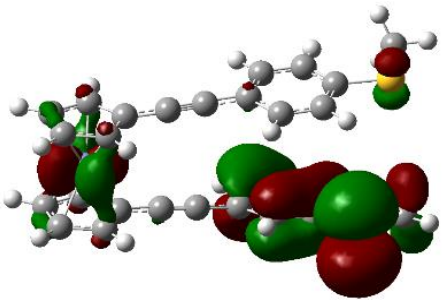

HOMO-3

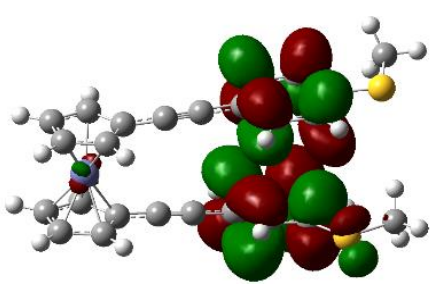

LUMO+4

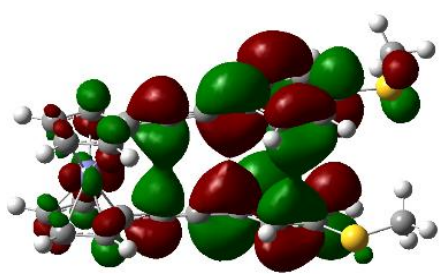

LUMO+1

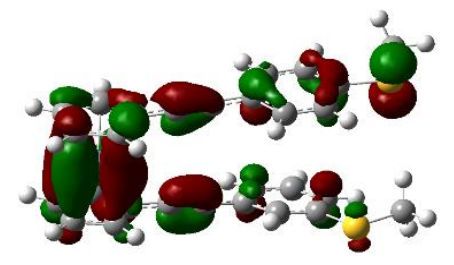

HOMO-1

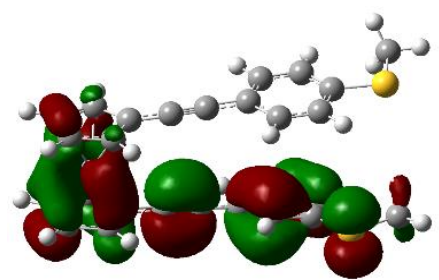

HOMO-4

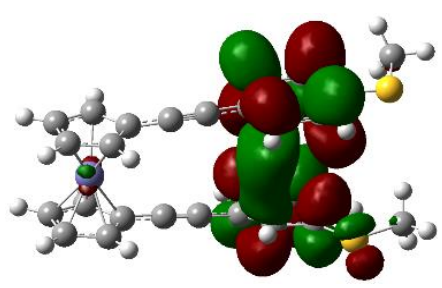

LUMO+3

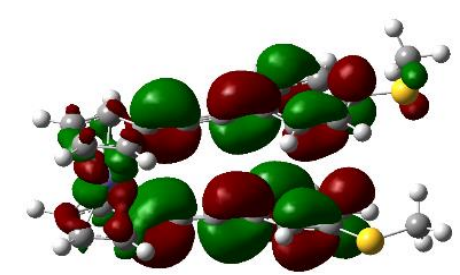

LUMO

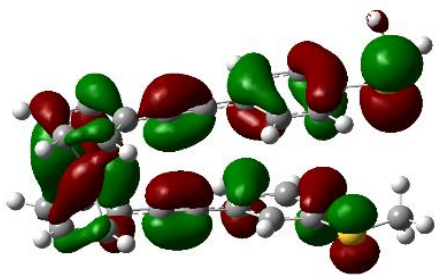

HOMO-2

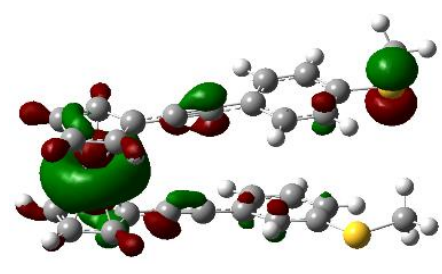

HOMO-5

Figure S100: Calculated iso-surfaces of HOMO-5 to LUMO-5 for compound 3cd

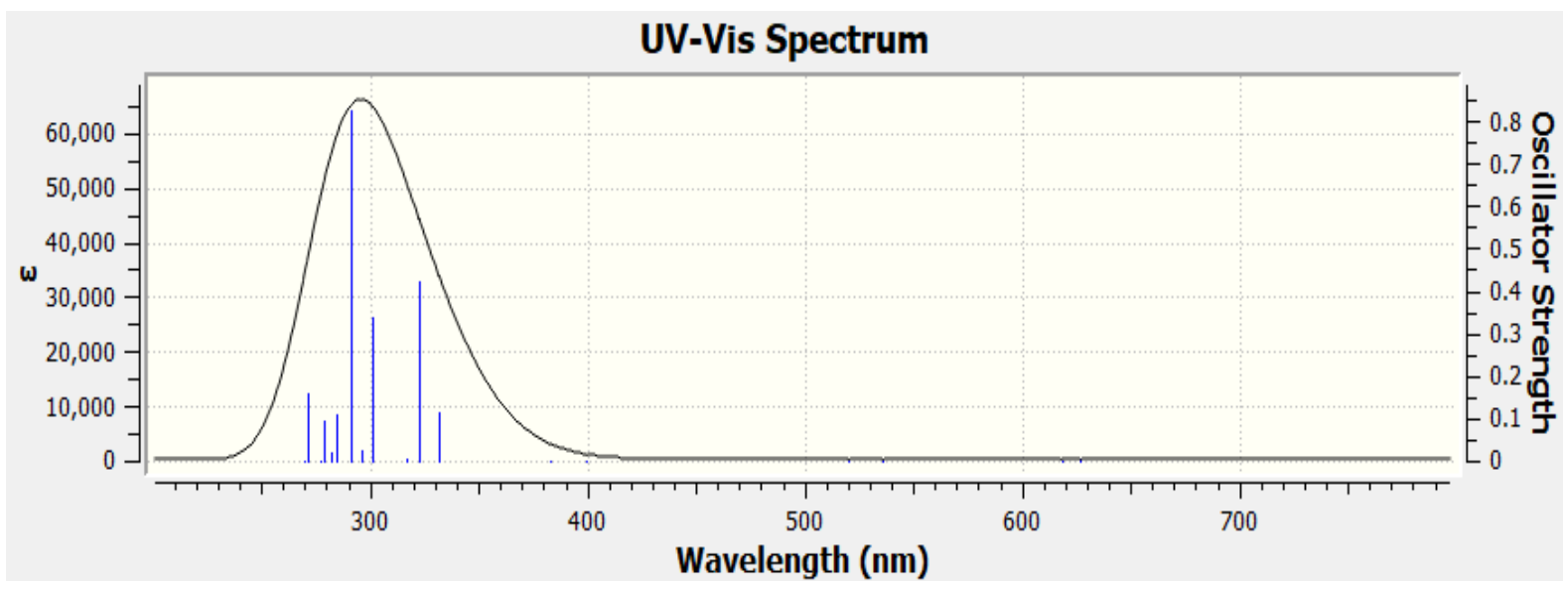

Figure S101: Calculated UV/Vis spectrum for compound 3cd 
Table S10: Assignment of transitions observed in the calculated UV/Vis spectra of compound 3cd

\begin{tabular}{|c|c|c|c|}
\hline $\begin{array}{l}\text { Wavelength } \\
\qquad(\mathrm{nm})\end{array}$ & $\begin{array}{l}\text { Oscillator } \\
\text { Strength } \\
\text { (f) }\end{array}$ & Assignment & $\begin{array}{l}\text { Excited } \\
\text { State }\end{array}$ \\
\hline 627.01 & 0.0037 & $\begin{array}{l}H-4 \rightarrow L(3.10 \%), H-4 \rightarrow L+5(3.22 \%), \\
H-3 \rightarrow L(5.07 \%), H-3 \rightarrow L+5(4.60 \%), \\
H-2 \rightarrow L(3.97 \%), H-2 \rightarrow L+3(11.90 \%), \\
H-2 \rightarrow L+5(3.97 \%), H-1 \rightarrow L(9.07 \%), \\
H-1 \rightarrow L+3(6.78 \%), H-1 \rightarrow L+5 \\
(7.56 \%), H \rightarrow L(19.46 \%), H \rightarrow L+5 \\
(13.59 \%)\end{array}$ & 1 \\
\hline 399.55 & 0.0011 & $\begin{array}{l}H-6 \rightarrow L(4.80 \%), H-6 \rightarrow L+5(3.80 \%), \\
H-5 \rightarrow L(18.47 \%), H-5 \rightarrow L+5 \\
(14.37 \%), H-4 \rightarrow L(4.45 \%), H-4 \rightarrow L+5 \\
(2.56 \%), H-3 \rightarrow L(2.47 \%), H-2 \rightarrow L+3 \\
(23.16 \%), H-1 \rightarrow L(3.58 \%), H-1 \rightarrow L+3 \\
(9.18 \%), H \rightarrow L(4.90 \%)\end{array}$ & 5 \\
\hline 331.66 & 0.1167 & $\begin{array}{l}H-4 \rightarrow \mathrm{L}(2.96 \%), \mathrm{H}-3 \rightarrow \mathrm{L}(3.01 \%), \mathrm{H}- \\
2 \rightarrow \mathrm{L}(4.65 \%), \mathrm{H}-1 \rightarrow \mathrm{L}(8.41 \%), \mathrm{H}- \\
1 \rightarrow \mathrm{L}+1(3.66 \%), \mathrm{H} \rightarrow \mathrm{L}(61.06 \%) \\
\mathrm{H} \rightarrow \mathrm{L}+1 \text { (7.69\%) }\end{array}$ & 7 \\
\hline 322.51 & 0.4243 & $\begin{array}{l}\mathrm{H}-4 \rightarrow \mathrm{L}(7.42 \%), \mathrm{H}-3 \rightarrow \mathrm{L}(3.31 \%), \\
\mathrm{H} \rightarrow \mathrm{L}(4.42 \%), \mathrm{H} \rightarrow \mathrm{L}+1 \text { (75.88\%) }\end{array}$ & 8 \\
\hline 300.93 & 0.3370 & $\begin{array}{l}H-3 \rightarrow L(52.67 \%), H-4 \rightarrow L+1 \\
(11.01 \%), H-2 \rightarrow L+1(6.32 \%), H-1 \rightarrow L \\
(14.03 \%), H \rightarrow L+1(4.50 \%)\end{array}$ & 10 \\
\hline 291.55 & 0.8245 & $\begin{array}{l}H-4 \rightarrow L(33.56 \%), H-3 \rightarrow L+1(8.81 \%), \\
H-2 \rightarrow L+(6.43 \%), H-2 \rightarrow L+1(3.09 \%), \\
H-1 \rightarrow L(18.57 \%), H-1 \rightarrow L+1(5.32 \%), \\
H \rightarrow L+1(6.43 \%), H \rightarrow L+5(2.90 \%)\end{array}$ & 12 \\
\hline 284.76 & 0.1092 & $\begin{array}{l}\mathrm{H}-4 \rightarrow \mathrm{L}(5.85 \%), \mathrm{H}-3 \rightarrow \mathrm{L}(18.70 \%), \mathrm{H}- \\
3 \rightarrow \mathrm{L}+1(66.70 \%)\end{array}$ & 13 \\
\hline 278.93 & 0.0965 & $\begin{array}{l}\mathrm{H}-4 \rightarrow \mathrm{L}(29.39 \%), \mathrm{H}-3 \rightarrow \mathrm{L}(4.10 \%), \mathrm{H}- \\
2 \rightarrow \mathrm{L}(2.30 \%), \mathrm{H}-2 \rightarrow \mathrm{L}+5(2.76 \%), \mathrm{H}- \\
1 \rightarrow \mathrm{L}(2.81 \%), \mathrm{H}-1 \rightarrow \mathrm{L}+5(4.57 \%), \\
\mathrm{H} \rightarrow \mathrm{L}(3.24 \%), \mathrm{H} \rightarrow \mathrm{L}+2(2.70 \%), \\
H \rightarrow L+5(33.82 \%)\end{array}$ & 15 \\
\hline 271.12 & 0.1607 & $\begin{array}{l}H-4 \rightarrow L(2.25 \%), H-4 \rightarrow L+1(83.77 \%), \\
H-3 \rightarrow L(2.11 \%)\end{array}$ & 17 \\
\hline
\end{tabular}




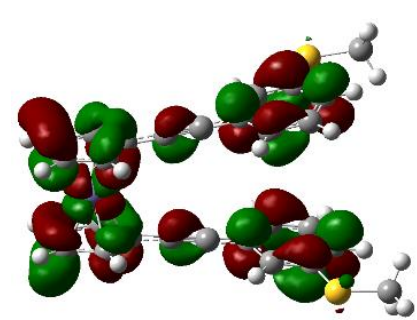

LUMO+5

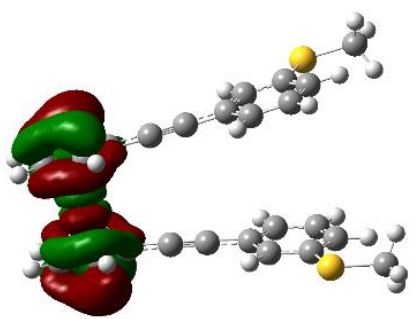

LUMO+2

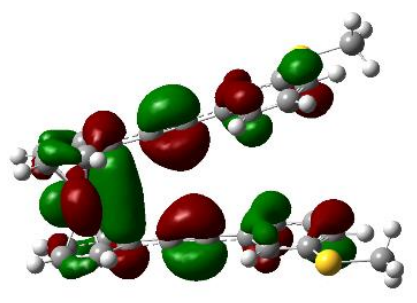

HOMO

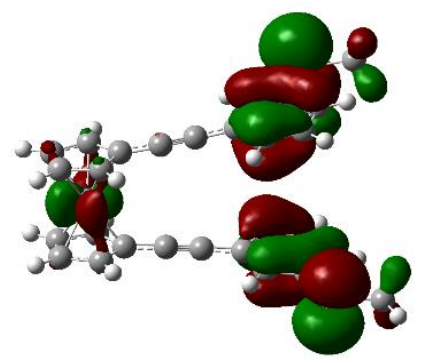

HOMO-3

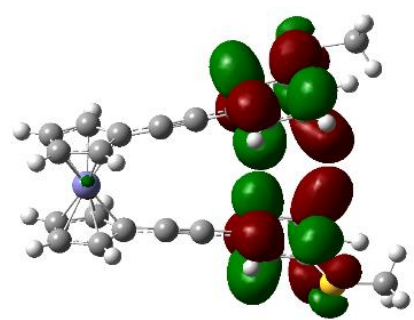

LUMO+4

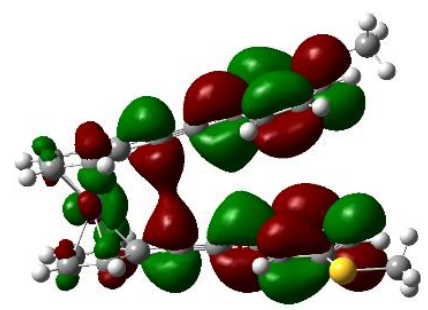

LUMO+1

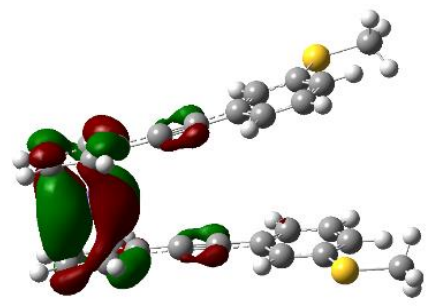

HOMO-1

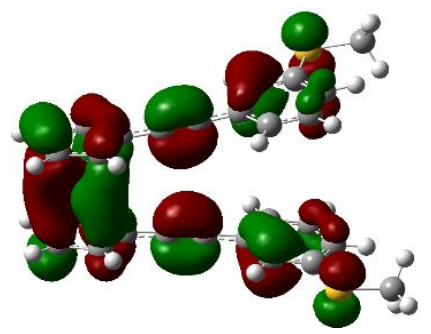

HOMO-4

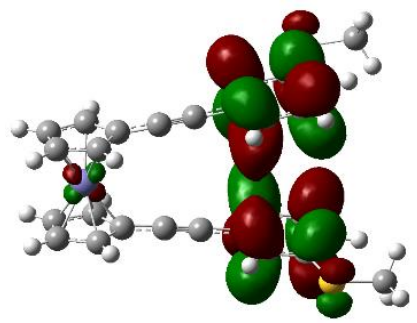

LUMO+3

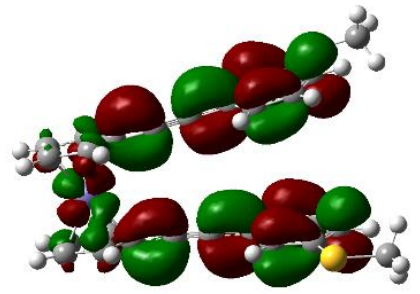

LUMO

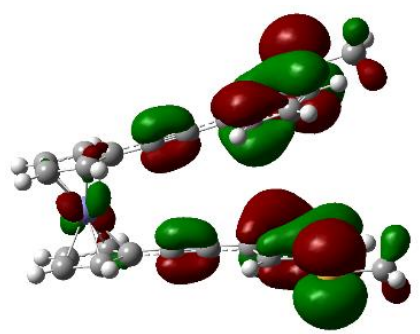

HOMO-2

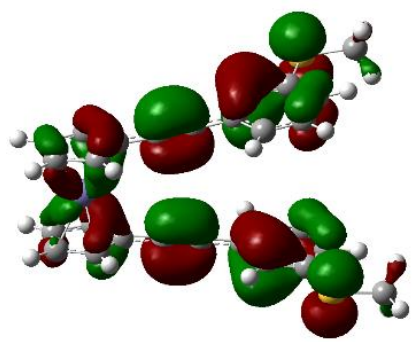

HOMO-5

Figure S102: Calculated iso-surfaces of HOMO-5 to LUMO-5 for compound 3dd

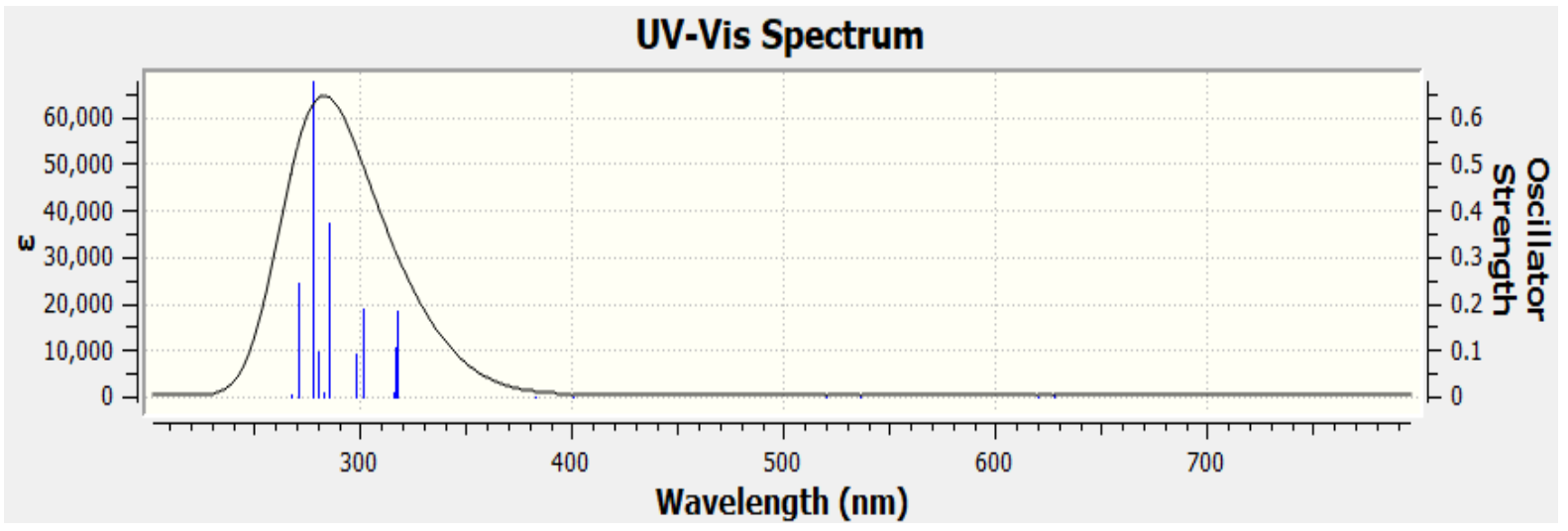

Figure S103: Calculated UV/Vis spectrum for compound 3dd 
Table S11: Assignment of transitions observed in the calculated UV/Vis spectra of compound 3dd

\begin{tabular}{|c|c|c|c|}
\hline $\begin{array}{l}\text { Wavelength } \\
\qquad(\mathrm{nm})\end{array}$ & $\begin{array}{l}\text { Oscillator } \\
\text { Strength } \\
\text { (f) }\end{array}$ & Assignment & $\begin{array}{c}\text { Excited } \\
\text { State }\end{array}$ \\
\hline 627.45 & 0.0043 & $\begin{array}{l}H-4 \rightarrow L(5.52 \%), H-4 \rightarrow L+5(5.92 \%), \\
H-3 \rightarrow L(3.93 \%), H-3 \rightarrow L+5(3.75 \%), \\
H-1 \rightarrow L+3(9.40 \%), H-1 \rightarrow L+4 \\
(9.88 \%), H \rightarrow L(30.51 \%), H \rightarrow L+5 \\
(24.11 \%)\end{array}$ & 1 \\
\hline 400.32 & 0.0012 & $\begin{array}{l}H-6 \rightarrow L(22.32 \%), H-6 \rightarrow L+5 \\
(18.26 \%), H-4 \rightarrow L(6.76 \%), H-4 \rightarrow L+5 \\
(3.97 \%), H-1 \rightarrow L+3(16.39 \%), H- \\
1 \rightarrow L+4(17.01 \%), H \rightarrow L(8.35 \%) \\
H \rightarrow L+5(2.32 \%)\end{array}$ & 5 \\
\hline 317.37 & 0.1843 & $\begin{array}{l}\mathrm{H}-4 \rightarrow \mathrm{L}(21.24 \%), \mathrm{H}-4 \rightarrow \mathrm{L}+5(2.11 \%), \\
\mathrm{H}-3 \rightarrow \mathrm{L}(14.18 \%), \mathrm{H}-1 \rightarrow \mathrm{L}+1(6.68 \%), \\
\mathrm{H} \rightarrow \mathrm{L}(42.64 \%), \mathrm{H} \rightarrow \mathrm{L}+5(5.40 \%)\end{array}$ & 7 \\
\hline 316.27 & 0.1040 & $\begin{array}{l}H-5 \rightarrow L(5.83 \%), H-2 \rightarrow L(6.94 \%) \\
H \rightarrow L+1(77.43 \%)\end{array}$ & 8 \\
\hline 301.15 & 0.1873 & $\begin{array}{l}H-3 \rightarrow L+1(10.67 \%), H-2 \rightarrow L \\
(68.37 \%), H-2 \rightarrow L+2(2.04 \%), H \rightarrow L+1 \\
(6.45 \%)\end{array}$ & 10 \\
\hline 298.43 & 0.0931 & $\begin{array}{l}H-5 \rightarrow L+1(3.80 \%), H-4 \rightarrow L(19.49 \%), \\
H-3 \rightarrow L(50.21 \%), H-2 \rightarrow L+1 \\
(12.06 \%), H \rightarrow L+2(3.26 \%)\end{array}$ & 11 \\
\hline 285.13 & 0.3746 & $\begin{array}{l}\mathrm{H}-5 \rightarrow \mathrm{L}(31.35 \%), \mathrm{H}-3 \rightarrow \mathrm{L}+1 \\
(43.97 \%), \mathrm{H}-2 \rightarrow \mathrm{L}(17.68 \%)\end{array}$ & 12 \\
\hline 280.43 & 0.0969 & $\begin{array}{l}H-5 \rightarrow L(2.15 \%), H-3 \rightarrow L+1(3.30 \%), \\
H-2 \rightarrow L(3.03 \%), H-1 \rightarrow L(35.68 \%), H- \\
1 \rightarrow L+5(43.01 \%)\end{array}$ & 14 \\
\hline 278.02 & 0.6779 & $\begin{array}{l}H-5 \rightarrow L(45.74 \%), H-4 \rightarrow L+1(4.18 \%), \\
H-3 \rightarrow L+1(28.74 \%), H-1 \rightarrow L(3.55 \%), \\
H-1 \rightarrow L+5(5.14 \%), H \rightarrow L+1(5.98 \%)\end{array}$ & 16 \\
\hline
\end{tabular}




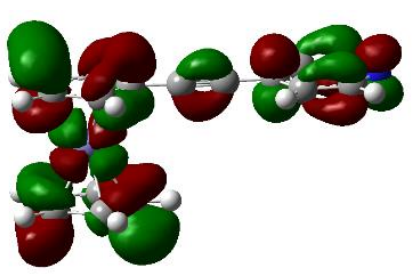

$\mathrm{LUMO}+3$

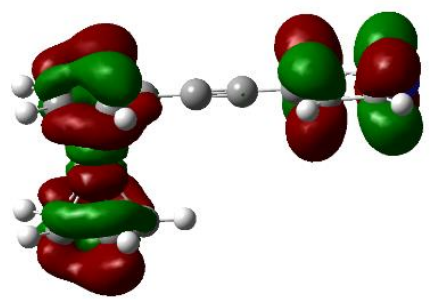

$\mathrm{LUMO}+1$

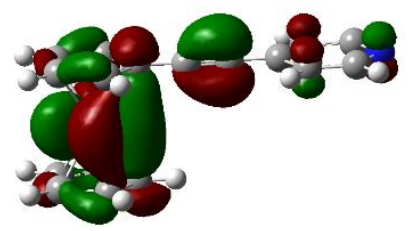

HOMO

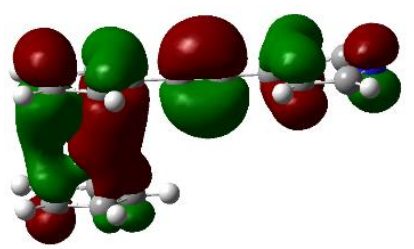

HOMO-2

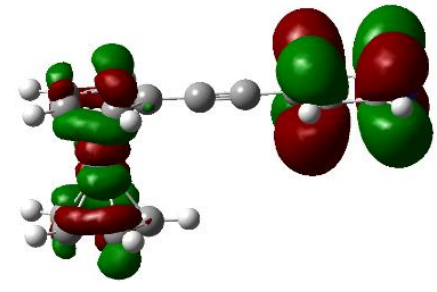

LUMO+2

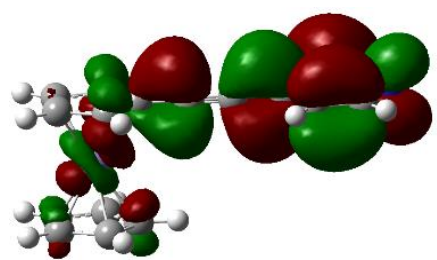

LUMO

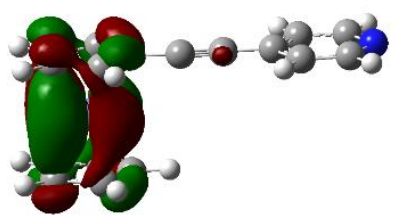

HOMO-1

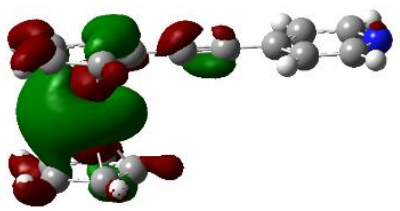

HOMO-3

Figure S104: Calculated iso-surfaces of HOMO-3 to LUMO-3 for compound 4a

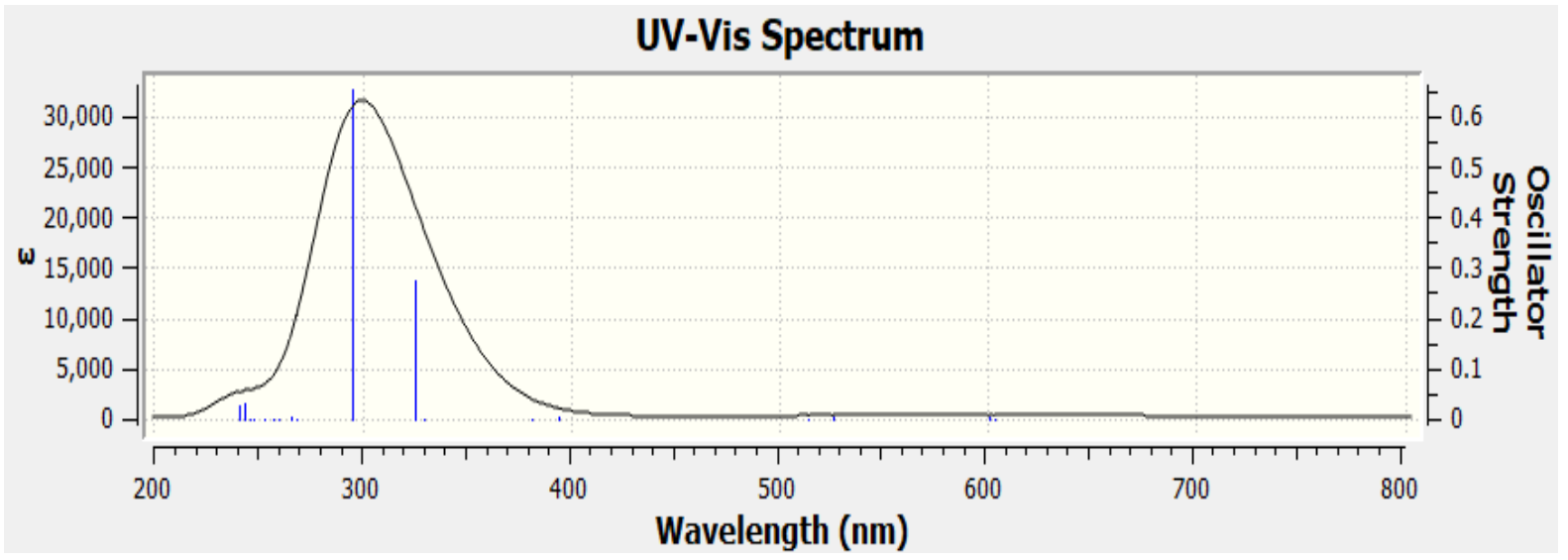

Figure S105: Calculated UV/Vis spectrum for compound 4a 
Table S12: Assignment of transitions observed in the calculated UV/Vis spectra of compound 4a

\begin{tabular}{|c|c|c|c|}
\hline $\begin{array}{c}\text { Wavelength } \\
(\mathrm{nm})\end{array}$ & $\begin{array}{l}\text { Oscillator } \\
\text { Strength } \\
\text { (f) }\end{array}$ & Assignment & $\begin{array}{l}\text { Excited } \\
\text { State }\end{array}$ \\
\hline 603.08 & 0.00006 & $\begin{array}{l}H-1 \rightarrow L(19.13 \%), H-1 \rightarrow L+3 \\
(47.72 \%), H \rightarrow L+2(28.08 \%)\end{array}$ & 1 \\
\hline 525.7 & 0.0022 & $\begin{array}{l}H-3 \rightarrow L(10.52 \%), H-3 \rightarrow L+3 \\
(36.41 \%), H-1 \rightarrow L+2(36.39 \%), H \rightarrow L \\
(3.85 \%), H \rightarrow L+3(8.44 \%)\end{array}$ & 3 \\
\hline 394.25 & 0.0046 & $\begin{array}{l}H-3 \rightarrow L(12.80 \%), H-3 \rightarrow L+3 \\
(28.81 \%), H-2 \rightarrow L(3.26 \%), H-2 \rightarrow L+3 \\
(4.33 \%), H-1 \rightarrow L+2(29.28 \%), H \rightarrow L \\
(13.99 \%), H \rightarrow L+3(7.15 \%)\end{array}$ & 5 \\
\hline 325.47 & 0.2729 & $\mathrm{H} \rightarrow \mathrm{L}(61.65 \%), \mathrm{H} \rightarrow \mathrm{L}+3$ (29.59\%) & 8 \\
\hline 294.74 & 0.6551 & $\begin{array}{l}\mathrm{H}-3 \rightarrow \mathrm{L}(2.82 \%), \mathrm{H}-2 \rightarrow \mathrm{L}(89.49 \%), \mathrm{H}- \\
\mathrm{L}+3(2.89 \%)\end{array}$ & 9 \\
\hline 243.21 & 0.0329 & $\begin{array}{l}H-10 \rightarrow L+1(2.17 \%), H-7-\rightarrow L \\
(44.43 \%), H-2 \rightarrow L+1(20.63 \%) \\
H \rightarrow L+1(29.87 \%)\end{array}$ & 17 \\
\hline 241.14 & 0.0257 & $\begin{array}{l}\mathrm{H}-5 \rightarrow \mathrm{L}(65.93 \%), \mathrm{H}-5 \rightarrow \mathrm{L}+3(6.49 \%) \\
\mathrm{H}-4 \rightarrow \mathrm{L}+2(4.88 \%), \mathrm{H} \rightarrow \mathrm{L} 4(14.29 \%)\end{array}$ & 18 \\
\hline
\end{tabular}




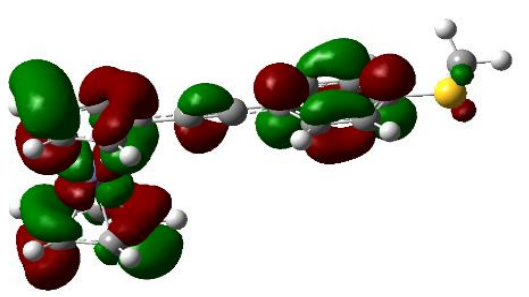

$\mathrm{LUMO}+3$

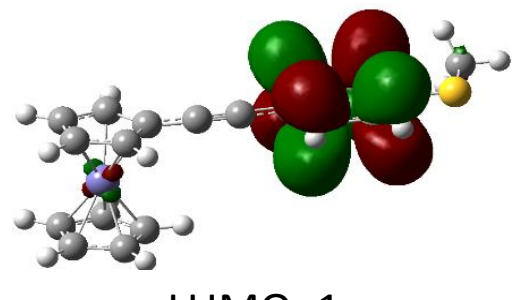

LUMO+1

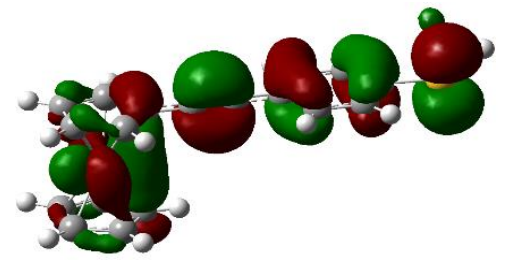

HOMO

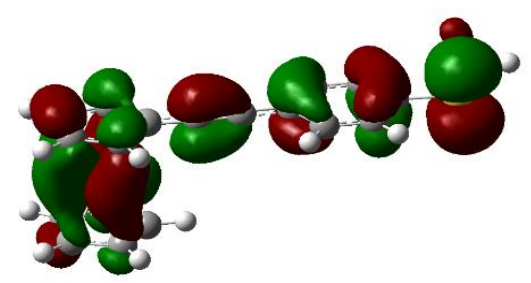

HOMO-3

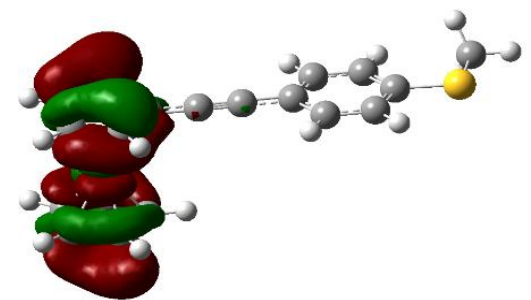

LUMO+2

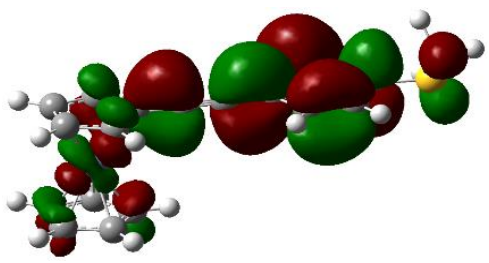

LUMO

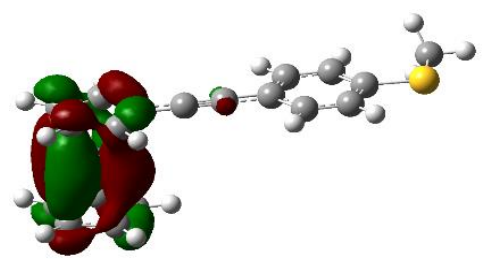

HOMO-1

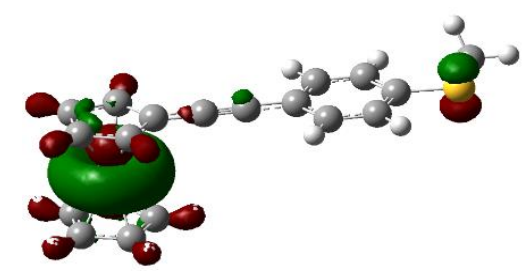

HOMO-4

Figure S106: Calculated iso-surfaces of HOMO-3 to LUMO-3 for compound 4c

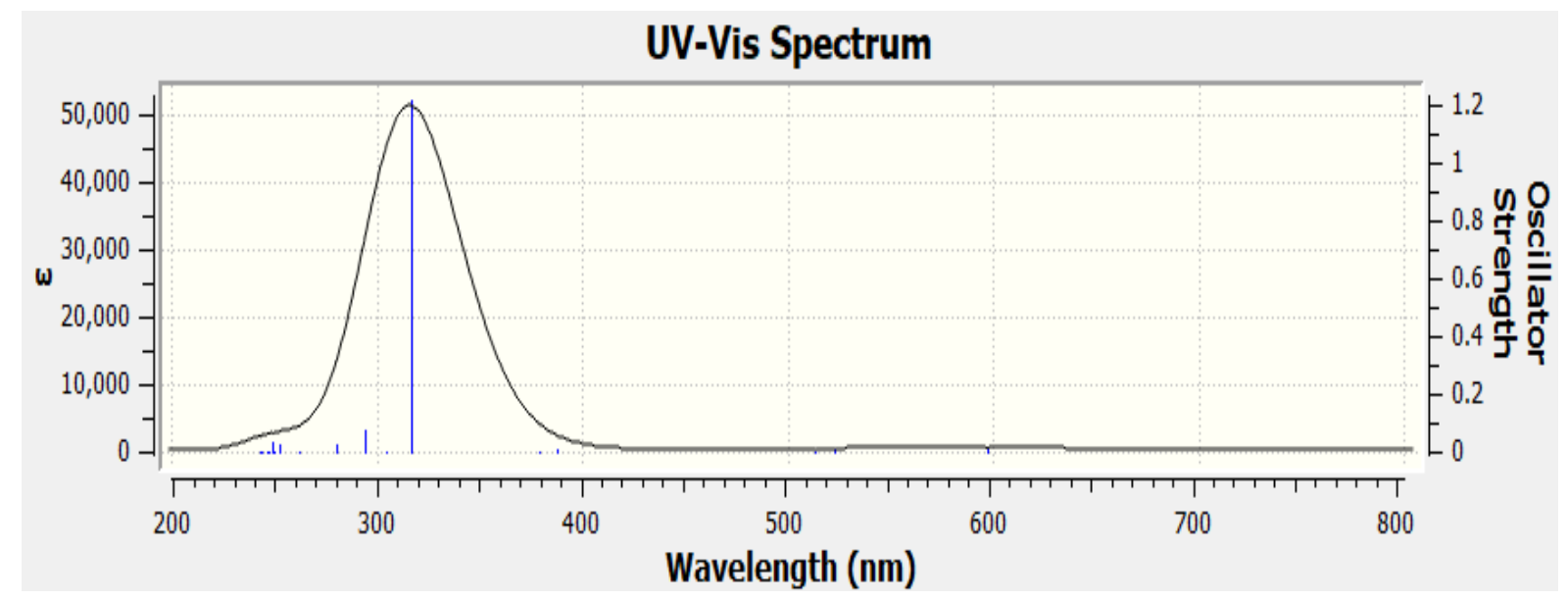

Figure S107: Calculated UV/Vis spectrum for compound 4c 
Table S13: Assignment of transitions observed in the calculated UV/Vis spectra of compound 4c

\begin{tabular}{|l|l|l|l|}
\hline $\begin{array}{c}\text { Wavelength } \\
(\mathrm{nm})\end{array}$ & $\begin{array}{c}\text { Oscillator } \\
\text { Strength } \\
\text { (f) }\end{array}$ & \multicolumn{1}{|c|}{ Assignment } & $\begin{array}{c}\text { Excited } \\
\text { State }\end{array}$ \\
\hline 597.2 & 0.0063 & $\begin{array}{l}\mathrm{H}-2 \rightarrow \mathrm{L}(9.04 \%) \mathrm{H}-2 \rightarrow \mathrm{L}+3(22.75 \%), \\
\mathrm{H}-1 \rightarrow \mathrm{L}+2(32.66 \%), \mathrm{H} \rightarrow \mathrm{L}(9.76 \%), \\
\mathrm{H} \rightarrow \mathrm{L}+3(20.87 \%)\end{array}$ & 2 \\
\hline 387.99 & 0.0026 & $\begin{array}{l}\mathrm{H}-3 \rightarrow \mathrm{L}(15.41 \%), \mathrm{H}-3 \rightarrow \mathrm{L}+3 \\
(30.89 \%), \mathrm{H}-2 \rightarrow \mathrm{L}(7.17 \%), \mathrm{H}-2 \rightarrow \mathrm{L}+3 \\
(7.70 \%), \mathrm{H}-1 \rightarrow \mathrm{L}+2(28.53 \%), \mathrm{H} \rightarrow \mathrm{L} \\
(4.56 \%), \mathrm{H} \rightarrow \mathrm{L}+3(3.87 \%)\end{array}$ & 5 \\
\hline 317.11 & 1.2129 & $\begin{array}{l}\mathrm{H}-2 \rightarrow \mathrm{L}(9.54 \%), \mathrm{H}-2 \rightarrow \mathrm{L}+3(3.28 \%), \\
\mathrm{H} \rightarrow \mathrm{L}(77.77 \%), \mathrm{H} \rightarrow \mathrm{L}+3(5.18 \%)\end{array}$ & 7 \\
\hline 294.12 & 0.0742 & $\begin{array}{l}\mathrm{H}-2 \rightarrow \mathrm{L}(65.13 \%), \mathrm{H}-2 \rightarrow \mathrm{L}+3(4.34 \%), \\
\mathrm{H} \rightarrow \mathrm{L}+3(20.81 \%)\end{array}$ & 9 \\
\hline 280.64 & 0.0220 & $\begin{array}{l}\mathrm{H}-6 \rightarrow \mathrm{L}(8.04 \%), \mathrm{H}-2 \rightarrow \mathrm{L}+1(10.80 \%), \\
\mathrm{H} \rightarrow \mathrm{L}+1(78.38 \%)\end{array}$ & 10 \\
\hline
\end{tabular}




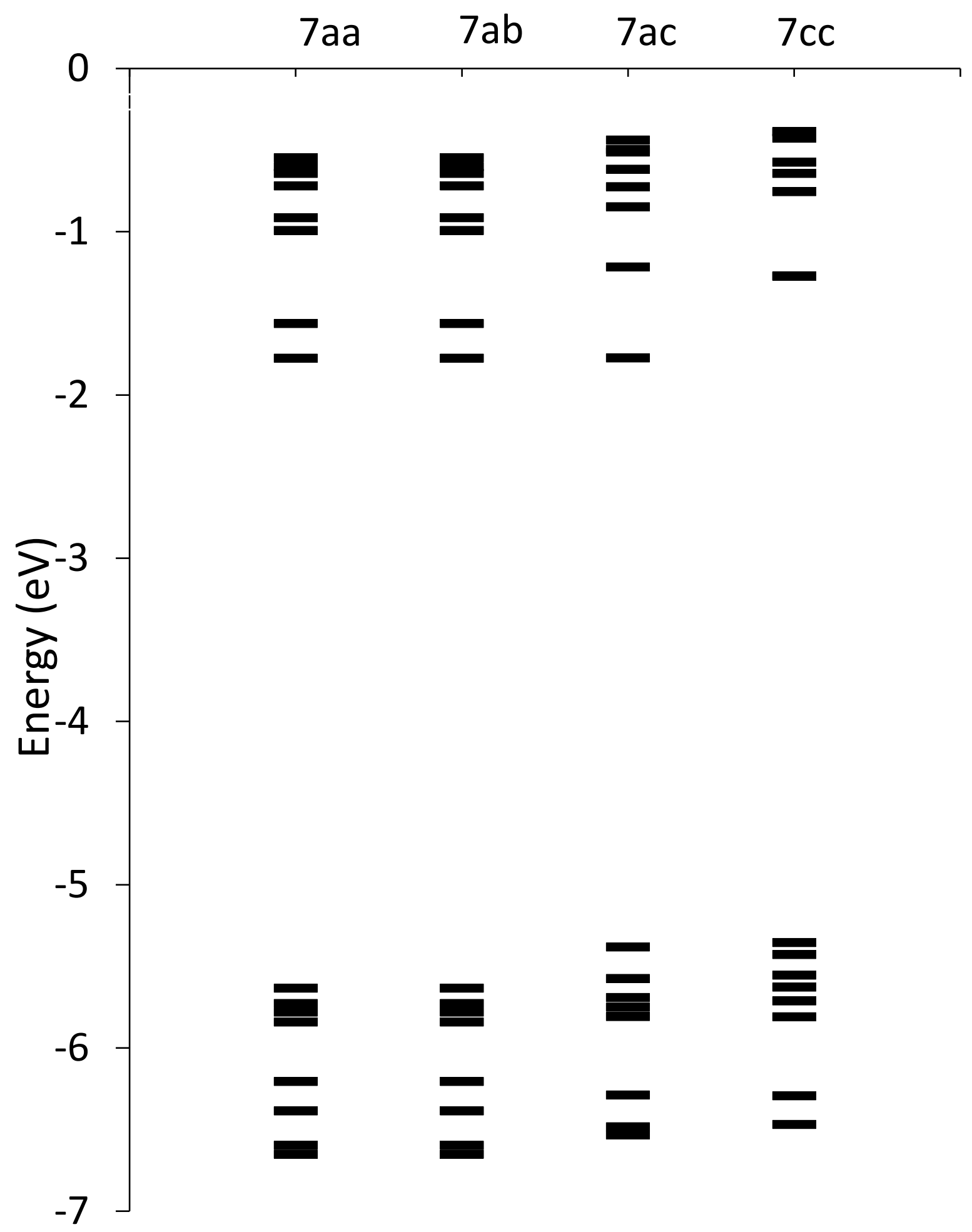

Figure S108: Energy level diagram for the frontier orbitals (HOMO-7 to LUMO+7) for 7xx 

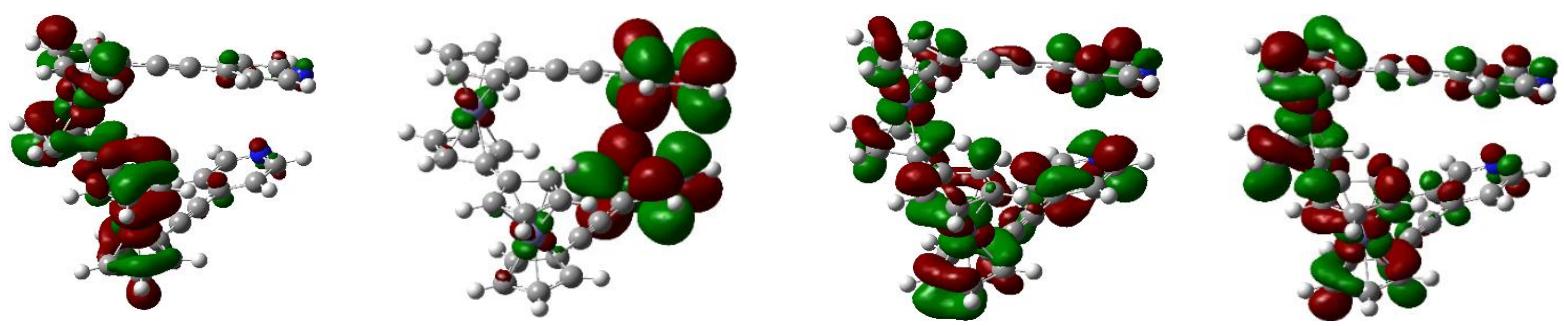

LUMO+7

LUMO+6

LUMO+5

LUMO+4
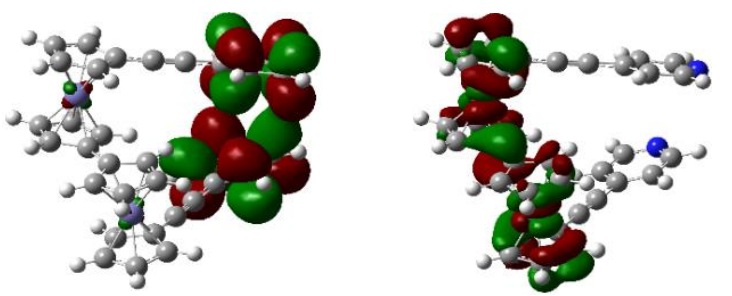

LUMO+2
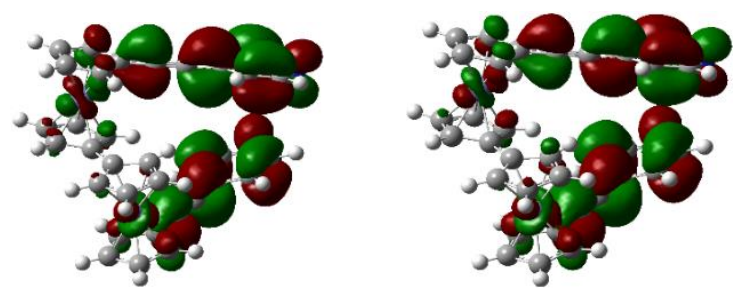

LUMO+3

LUMO+1

LUMO

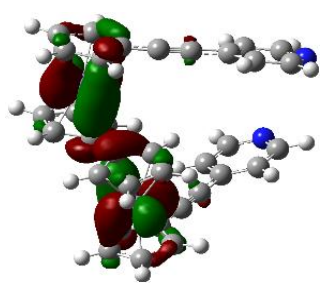

HOMO

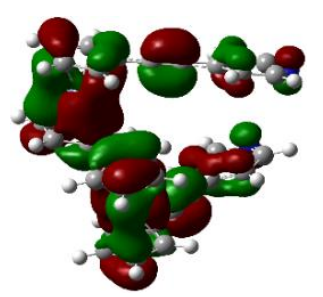

HOMO-4
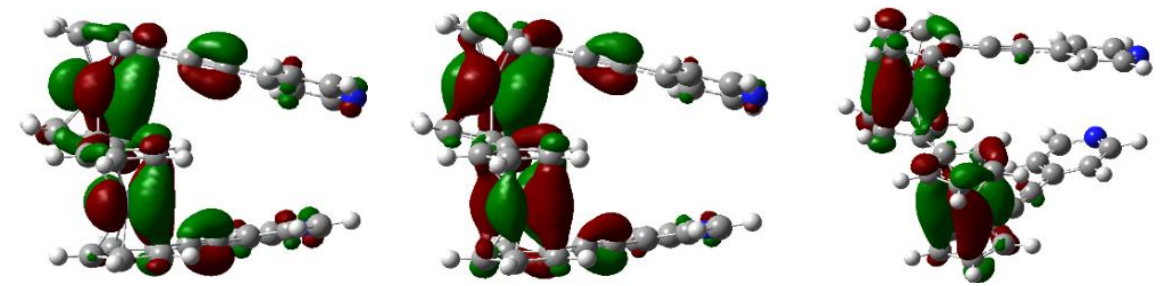

HOMO-

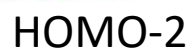

HOMO-3

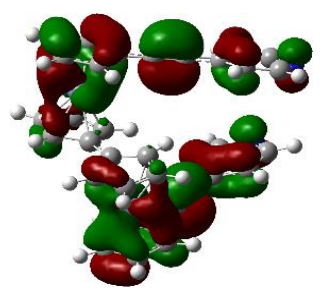

HOMO-5
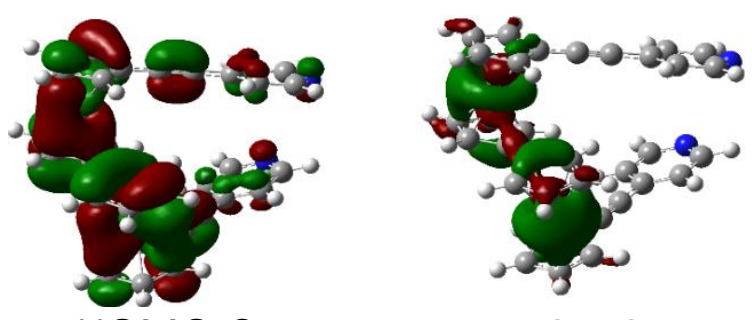

HOMO-6

HOMO-7

Figure S109: Calculated iso-surfaces of HOMO-7 to LUMO-7 for compound 7aa

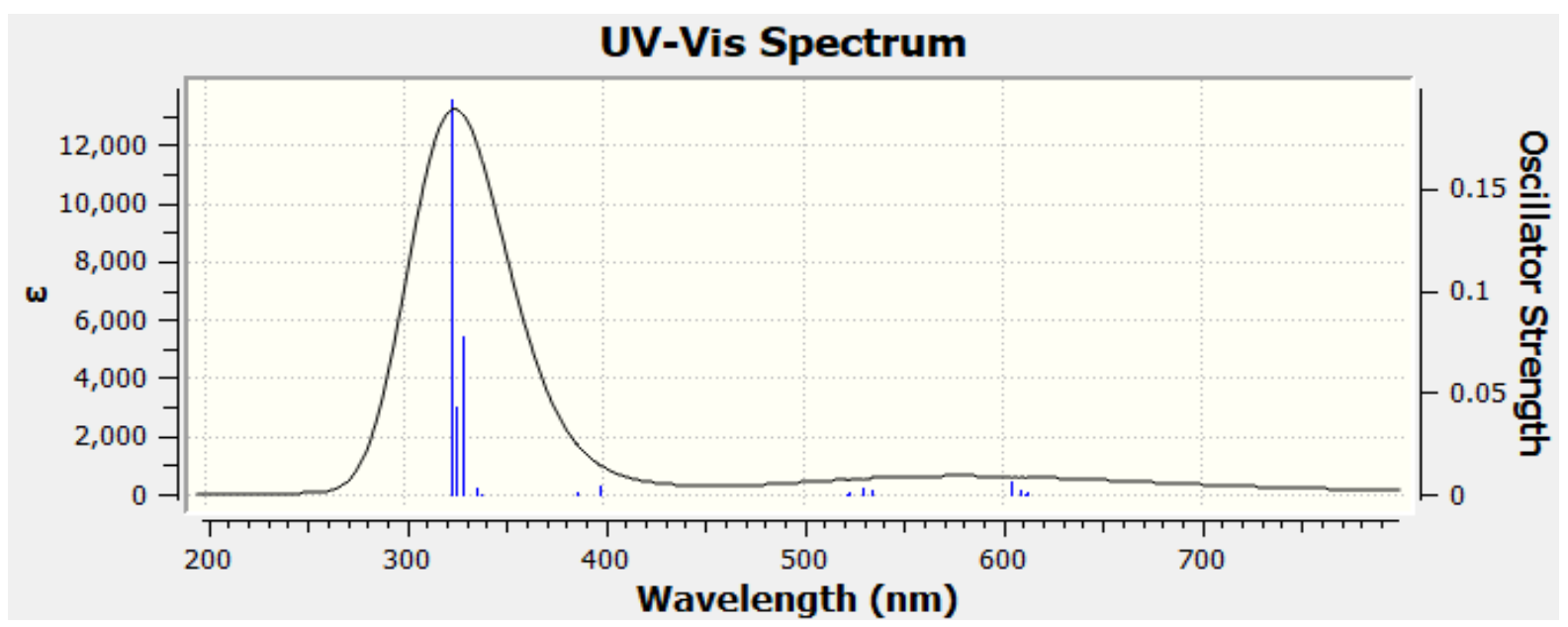

Figure S110: Calculated UV/Vis spectrum for compound 7aa 
Table S14: Assignment of transitions observed in the calculated UV/Vis spectra of compound 7aa

\begin{tabular}{|c|c|c|c|}
\hline $\begin{array}{l}\text { Wavelength } \\
\qquad(\mathrm{nm})\end{array}$ & $\begin{array}{l}\text { Oscillator } \\
\text { Strength } \\
\text { (f) }\end{array}$ & Assignment & $\begin{array}{c}\text { Excited } \\
\text { State }\end{array}$ \\
\hline 605 & 0.0066 & $\begin{array}{l}\mathrm{H}-3 \rightarrow \mathrm{L}+3(14.92 \%), \mathrm{H}-2 \rightarrow \mathrm{L}(6.11 \%) \\
\mathrm{H}-2 \rightarrow \mathrm{L}+5(16.84 \%), \mathrm{H}-2 \rightarrow \mathrm{L}+6 \\
(2.18 \%), \mathrm{H}-1 \rightarrow \mathrm{L}+1(7.95 \%), \mathrm{H}- \\
2 \rightarrow \mathrm{L}+3(2.44 \%), \mathrm{H}-2 \rightarrow \mathrm{L}+6(16.91 \%) \\
\mathrm{H} \rightarrow \mathrm{L}+7(15.19 \%)\end{array}$ & 4 \\
\hline 531 & 0.0034 & $\begin{array}{l}H-8 \rightarrow L+1(5.17 \%), H-8 \rightarrow L+6 \\
(14.65 \%), H-7 \rightarrow L(4.74 \%), H-7 \rightarrow L+5 \\
(13.71 \%), H-7 \rightarrow L+7(4.65 \%), H- \\
3 \rightarrow L+3(16.08 \%), H-2 \rightarrow L+7(6.49 \%), \\
H-1 \rightarrow L+1(2.51 \%), H-1 \rightarrow L+6 \\
(4.75 \%), H \rightarrow L+5(6.03 \%), H \rightarrow L+7 \\
(4.94 \%)\end{array}$ & 6 \\
\hline 398 & 0.0042 & $\begin{array}{l}H-8 \rightarrow L+1(6.67 \%), H-8 \rightarrow L+6 \\
(12.99 \%), H-7 \rightarrow L(6.38 \%), H-7 \rightarrow L+5 \\
(10.37 \%), H-7 \rightarrow L+7(3.35 \%), H- \\
3 \rightarrow L+3(14.11 \%), H-2 \rightarrow L(3.16 \%), H- \\
2 \rightarrow L+7(4.58 \%), H-1 \rightarrow L+1(6.11 \%) \\
H-1 \rightarrow L+6(2.91 \%), H \rightarrow L(5.19 \%) \\
H \rightarrow L+5(7.05 \%), H \rightarrow L+7(2.37 \%)\end{array}$ & 10 \\
\hline 329 & 0.0774 & $\begin{array}{l}\mathrm{H}-4 \rightarrow \mathrm{L}+1(2.27 \%), \mathrm{H}-3 \rightarrow \mathrm{L}(6.98 \%) \\
\mathrm{H}-2 \rightarrow \mathrm{L}+6(2.59 \%), \mathrm{H}-1 \rightarrow \mathrm{L}(60.45 \%) \\
\mathrm{H}-1 \rightarrow \mathrm{L}+5(4.98 \%), \mathrm{H} \rightarrow \mathrm{L}+6(8.80 \%)\end{array}$ & 15 \\
\hline 327 & 0.0429 & $\begin{array}{l}H-3 \rightarrow L+1(9.25 \%), H-3 \rightarrow L+6 \\
(2.06 \%), H-2 \rightarrow L(58.40 \%), H-2 \rightarrow L+5 \\
(4.34 \%), H-1 \rightarrow L+1(5.78 \%), H- \\
1 \rightarrow L+6(4.74 \%), H \rightarrow L(6.90 \%)\end{array}$ & 16 \\
\hline 324 & 0.1933 & $\begin{array}{l}H-2 \rightarrow L+5(4.19 \%), H-2 \rightarrow L+7 \\
(3.09 \%), H-1 \rightarrow L+1(71.92 \%), H- \\
1 \rightarrow L+6(6.93 \%), H \rightarrow L+5(3.26 \%)\end{array}$ & 18 \\
\hline
\end{tabular}




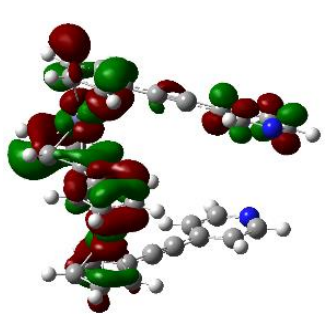

LUMO+7

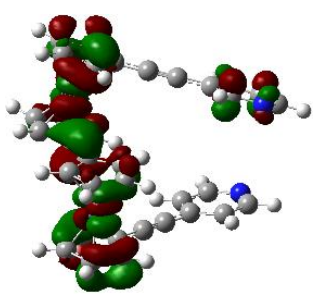

$\mathrm{LUMO}+3$

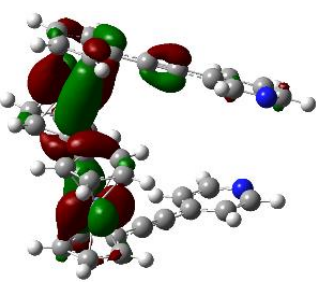

HOMO

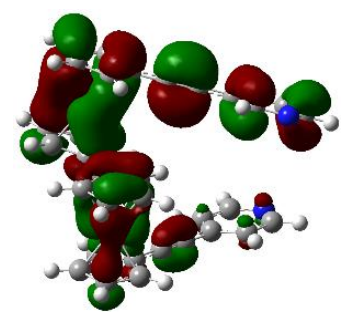

HOMO-4

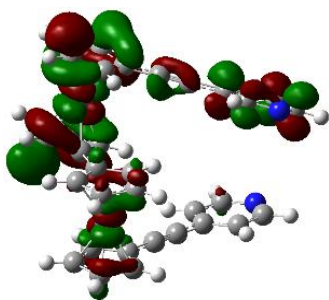

LUMO+6

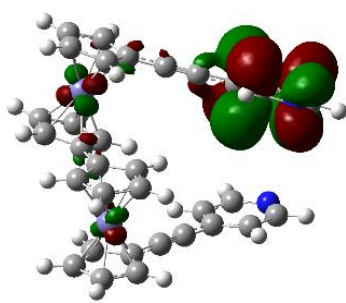

LUMO+2

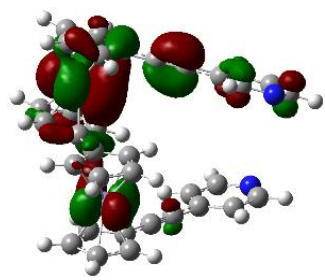

HOMO-

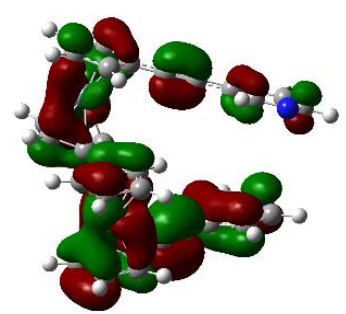

HOMO-5

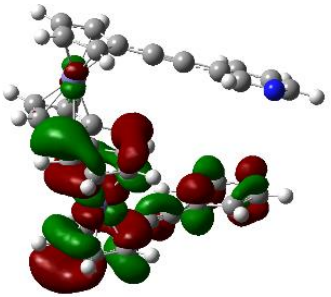

LUMO+5

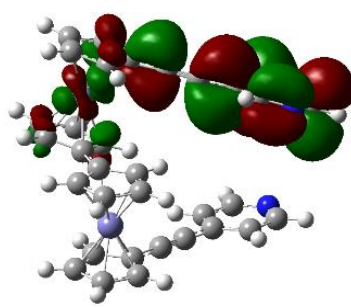

LUMO+1

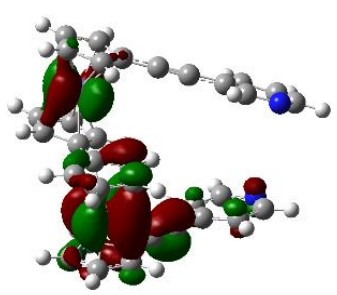

HOMO-2

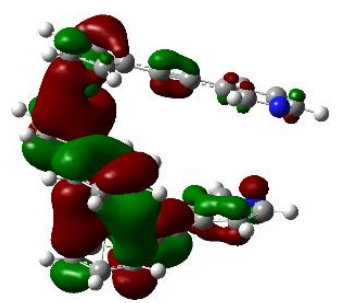

HOMO-6

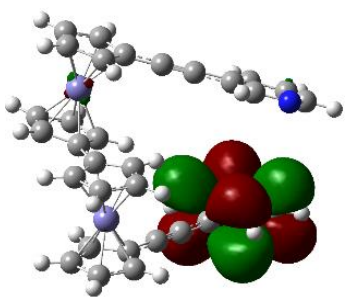

LUMO+4

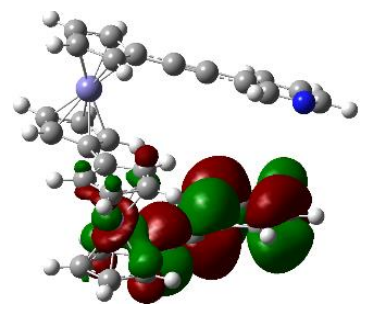

LUMO

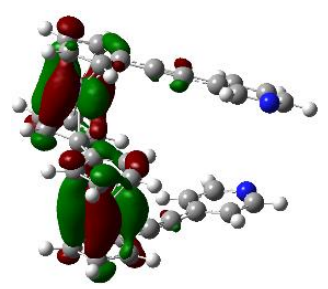

HOMO-3

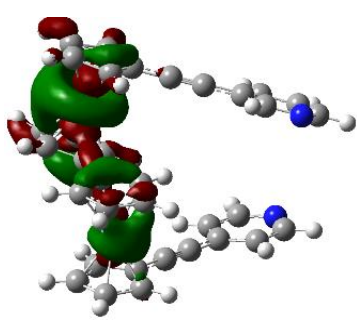

HOMO-7

Figure S111: Calculated iso-surfaces of HOMO-7 to LUMO-7 for compound 7ab

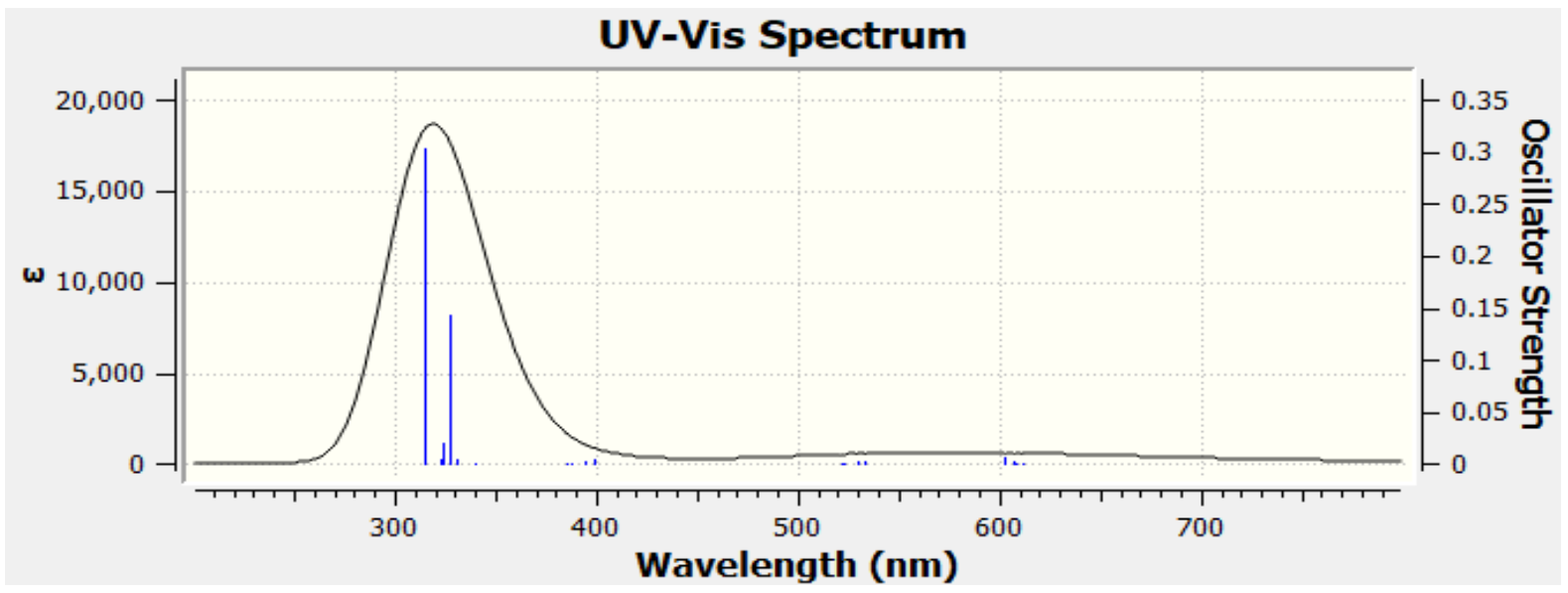

Figure S112: Calculated UV/Vis spectrum for compound 7ab 
Table S15: Assignment of transitions observed in the calculated UV/Vis spectra of compound 7ab

\begin{tabular}{|c|c|c|c|}
\hline $\begin{array}{c}\text { Wavelength } \\
(\mathrm{nm})\end{array}$ & $\begin{array}{l}\text { Oscillator } \\
\text { Strength } \\
\text { (f) }\end{array}$ & Assignment & $\begin{array}{c}\text { Excited } \\
\text { State }\end{array}$ \\
\hline 603 & 0.0058 & $\begin{array}{l}H-3 \rightarrow L+3(5.18 \%), H-3 \rightarrow L+4 \\
(8.13 \%), H-3 \rightarrow L+6(2.23 \%), H-2 \rightarrow L \\
(2.97 \%), H-2 \rightarrow L+5(7.70 \%), H- \\
2 \rightarrow L+6(57.07 \%), H-1 \rightarrow L+1(7.49 \%), \\
H-1 \rightarrow L+5(2.03 \%), H-1 \rightarrow L+6 \\
(18.62 \%), H \rightarrow L+7(15.76 \%)\end{array}$ & 4 \\
\hline 529 & 0.0033 & $\begin{array}{l}H-8 \rightarrow L(2.49 \%), H-8 \rightarrow L+5(8.87 \%), \\
H-8 \rightarrow L+6(2.20 \%), H-7 \rightarrow L+1 \\
(6.16 \%), H-7 \rightarrow L+6(13.08 \%), H- \\
7 \rightarrow L+7(7.29 \%), H-3 \rightarrow L+3(5.80 \%) \\
H-3 \rightarrow L+4(9.38 \%), H-2 \rightarrow L+7 \\
(5.41 \%), H-1 \rightarrow L+1(2.75 \%), H- \\
1 \rightarrow L+6(2.89 \%), H-1 \rightarrow L+7(3.10 \%) \\
H \rightarrow L+6(7.15 \%), H \rightarrow L+7(2.25 \%)\end{array}$ & 6 \\
\hline 399 & 0.0035 & $\begin{array}{l}H-8 \rightarrow L(11.28 \%), H-8 \rightarrow L+5 \\
(23.08 \%), H-7 \rightarrow L+5(3.98 \%), H- \\
3 \rightarrow L+3(3.12 \%), H-3 \rightarrow L+4(4.94 \%), \\
H-3 \rightarrow L+7(3.71 \%), H-2 \rightarrow L(7.06 \%), \\
H-2 \rightarrow L+4(2.64 \%), H-1 \rightarrow L(3.01 \%), \\
H-1 \rightarrow L+5(2.08 \%), H \rightarrow L(4.59 \%), \\
H \rightarrow L+4(2.18 \%), H \rightarrow L+5(4.55 \%)\end{array}$ & 9 \\
\hline 327 & 0.1427 & $\begin{array}{l}H-2 \rightarrow L(39.67 \%), H-2 \rightarrow L+5 \\
(17.07 \%), H-1 \rightarrow L(12.18 \%), H- \\
1 \rightarrow L+5(4.36 \%), H \rightarrow L(4.77 \%) \\
H \rightarrow L+5(3.60 \%)\end{array}$ & 15 \\
\hline 315 & 0.3027 & $\begin{array}{l}H-4 \rightarrow L+1(2.63 \%), H-2 \rightarrow L(3.40 \%), \\
H-2 \rightarrow L+1(3.07 \%), H-1 \rightarrow L+1 \\
(47.91 \%), H-1 \rightarrow L+6(10.23 \%), H- \\
1 \rightarrow L+7(2.60 \%), H \rightarrow L+1(10.27 \%) \\
H \rightarrow L+6(5.12 \%)\end{array}$ & 18 \\
\hline
\end{tabular}




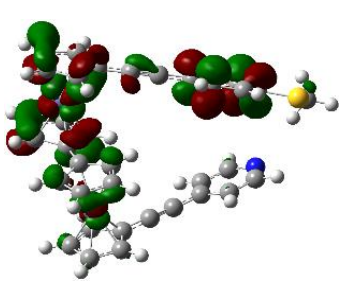

LUMO+7

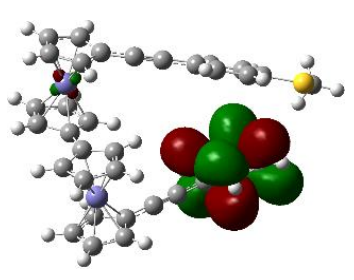

LUMO+3

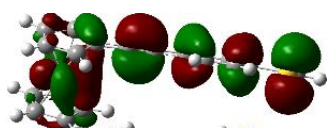

-

HOMO

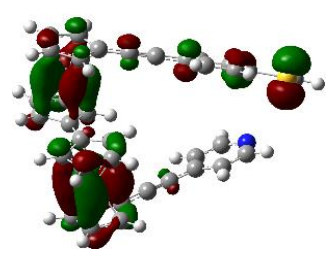

HOMO-4

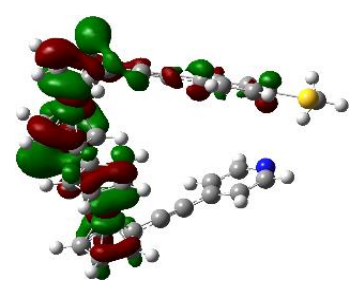

LUMO+6

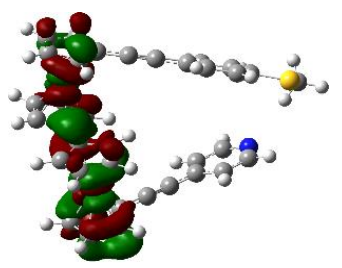

LUMO+2

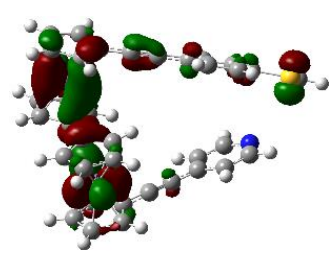

HOMO-

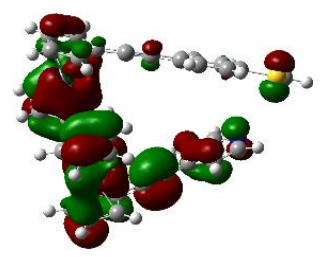

HOMO-5

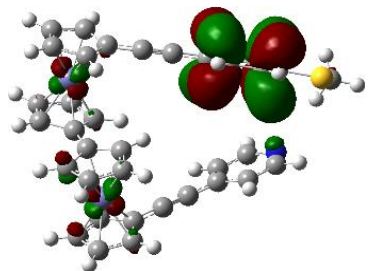

LUMO+5

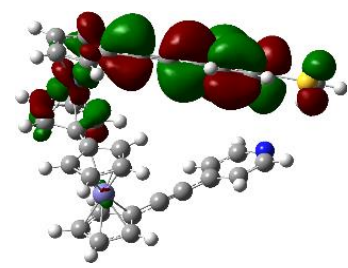

LUMO+1

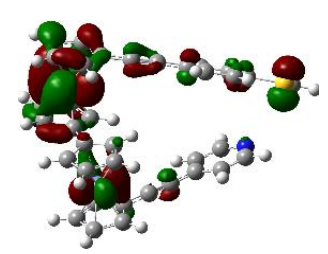

HOMO-2

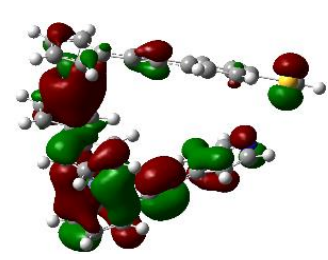

HOMO-6

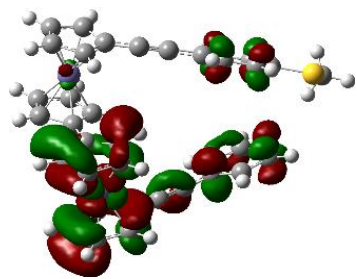

LUMO+4

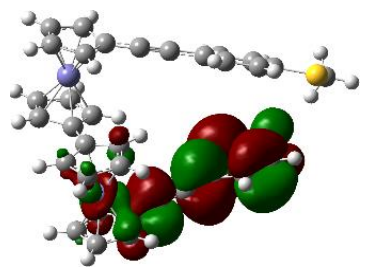

LUMO

Figure S113: Calculated iso-surfaces of HOMO-7 to LUMO-7 for compound 7ac

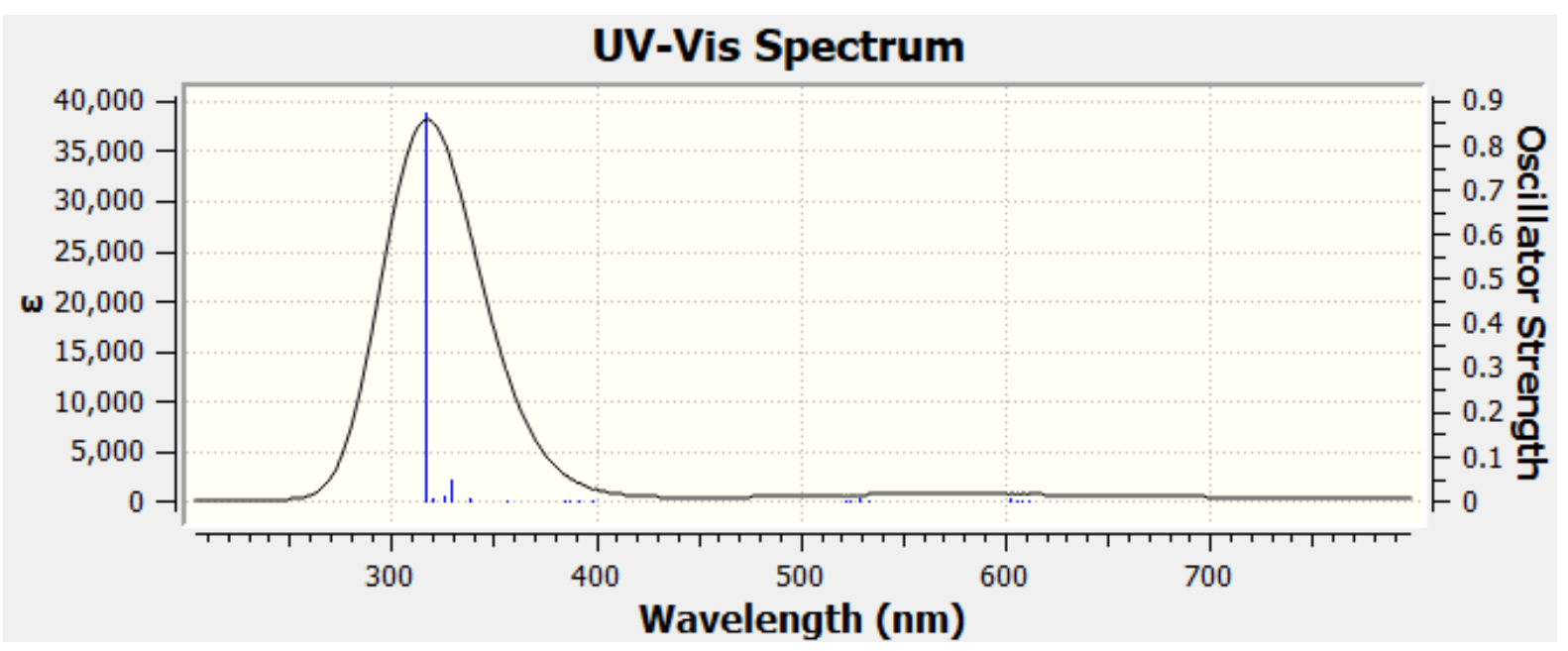

Figure S114: Calculated UV/Vis spectrum for compound 7ac 
Table S16: Assignment of transitions observed in the calculated UV/Vis spectra of compound 7ac

\begin{tabular}{|c|c|c|c|}
\hline $\begin{array}{c}\text { Wavelength } \\
(\mathrm{nm})\end{array}$ & $\begin{array}{l}\text { Oscillator } \\
\text { Strength } \\
\text { (f) }\end{array}$ & Assignment & $\begin{array}{c}\text { Excited } \\
\text { State }\end{array}$ \\
\hline 602 & 0.0048 & $\begin{array}{l}H-4 \rightarrow L+1(2.93 \%), H-4 \rightarrow L+3 \\
(7.86 \%), H-4 \rightarrow L+7(4.38 \%), H- \\
3 \rightarrow L+1(2.14 \%), H-3 \rightarrow L+5(4.37 \%), \\
H-3 \rightarrow L+6(3.64 \%), H-2 \rightarrow L+1 \\
(3.85 \%), H-3 \rightarrow L+3(5.20 \%), H- \\
3 \rightarrow L+6(14.81 \%), H-1 \rightarrow L+6(6.41 \%), \\
H-1 \rightarrow L+7(3.54 \%), H \rightarrow L+1(4.38 \%), \\
H \rightarrow L+3(4.22 \%), H \rightarrow L+7(10.09 \%)\end{array}$ & 4 \\
\hline 528 & 0.0036 & $\begin{array}{l}H-10 \rightarrow L+5(5.75 \%), H-7 \rightarrow L+1 \\
(8.09 \%), H-7 \rightarrow L+6(11.91 \%), H- \\
7 \rightarrow L+7(9.18 \%), H-4 \rightarrow L+3(3.57 \%), \\
H-4 \rightarrow L+6(2.46 \%), H-3 \rightarrow L+3 \\
(5.09 \%), H-2 \rightarrow L+3(3.76 \%), H- \\
2 \rightarrow L+7(7.04 \%), H-1 \rightarrow L+3(3.15 \%), \\
H-1 \rightarrow L+7(3.41 \%), H \rightarrow L+1(2.19 \%), \\
H \rightarrow L+6(6.68 \%)\end{array}$ & 6 \\
\hline 399 & 0.0032 & $\begin{array}{l}H-10 \rightarrow L(10.70 \%), H-10 \rightarrow L+5 \\
(24.00 \%), H-9 \rightarrow L+5(3.42 \%), H- \\
4 \rightarrow L+3(5.39 \%), H-3 \rightarrow L(3.43 \%), H- \\
3 \rightarrow L+3(8.72 \%), H-3 \rightarrow L+7(3.04 \%) \\
H-2 \rightarrow L(3.26 \%), H-1 \rightarrow L(7.91 \%), H- \\
1 \rightarrow L+3(3.14 \%), H-1 \rightarrow L+5(5.11 \%)\end{array}$ & 9 \\
\hline 317 & 0.8716 & $\begin{array}{l}H-4 \rightarrow L(4.51 \%), H-4 \rightarrow L+1(3.01 \%), \\
H-3 \rightarrow L(2.03 \%), H-2 \rightarrow L(4.09 \%), H- \\
1 \rightarrow L+1(7.98 \%), H \rightarrow L+1(59.98 \%) \\
H \rightarrow L+6(2.32 \%)\end{array}$ & 18 \\
\hline
\end{tabular}



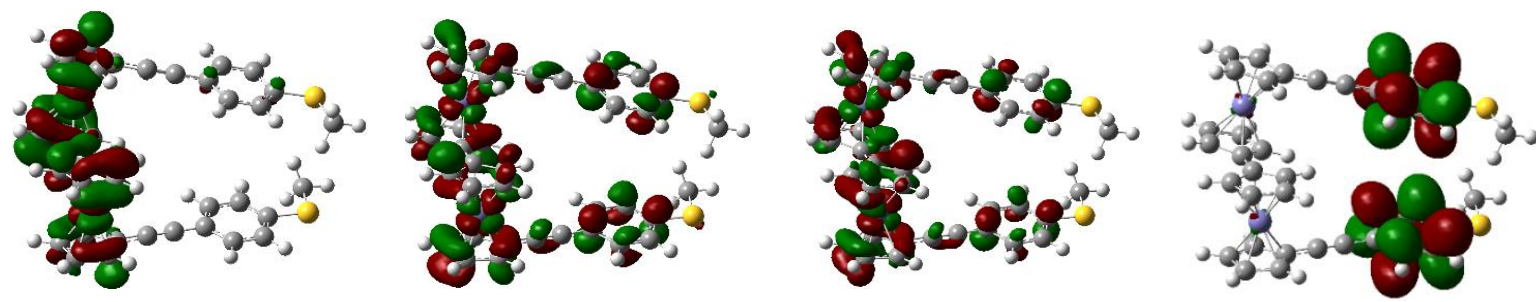

LUMO+7

LUMO+6

LUMO+5

LUMO+4
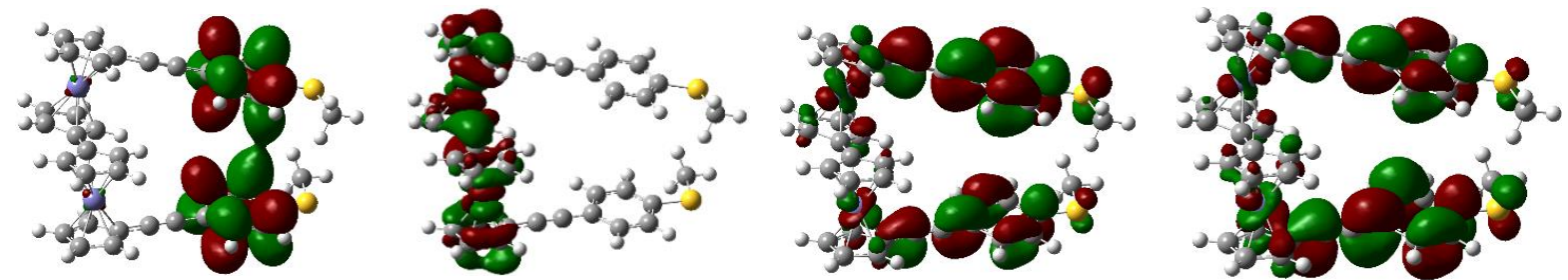

LUMO+3

LUMO+2

LUMO+1

LUMO

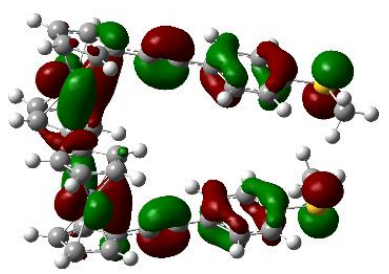

HOMO

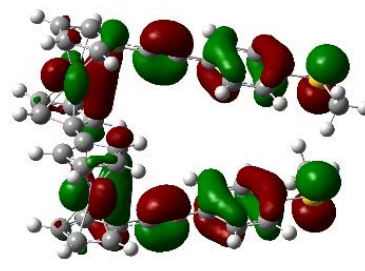

HOMO-

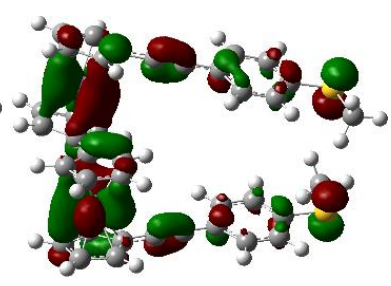

HOMO-2

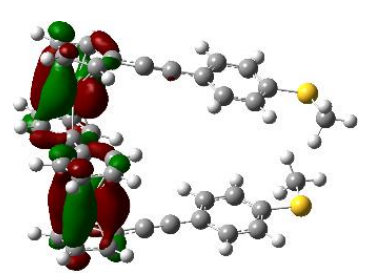

HOMO-3

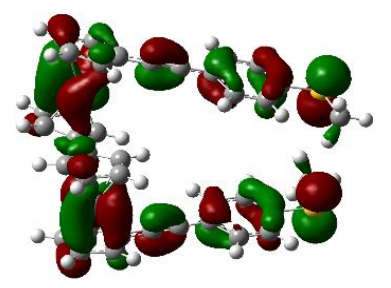

HOMO-4

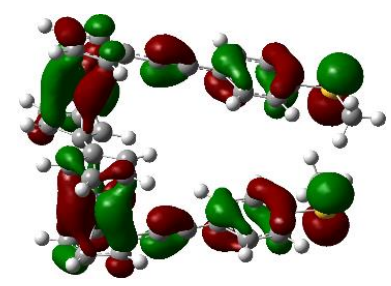

HOMO-5

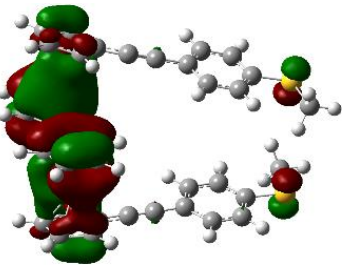

HOMO-6

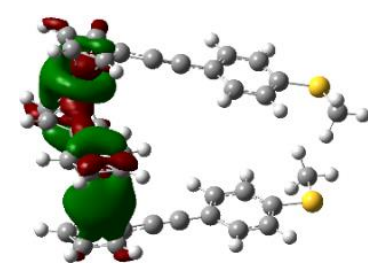

HOMO-7

Figure S115: Calculated iso-surfaces of HOMO-7 to LUMO-7 for compound 7cc

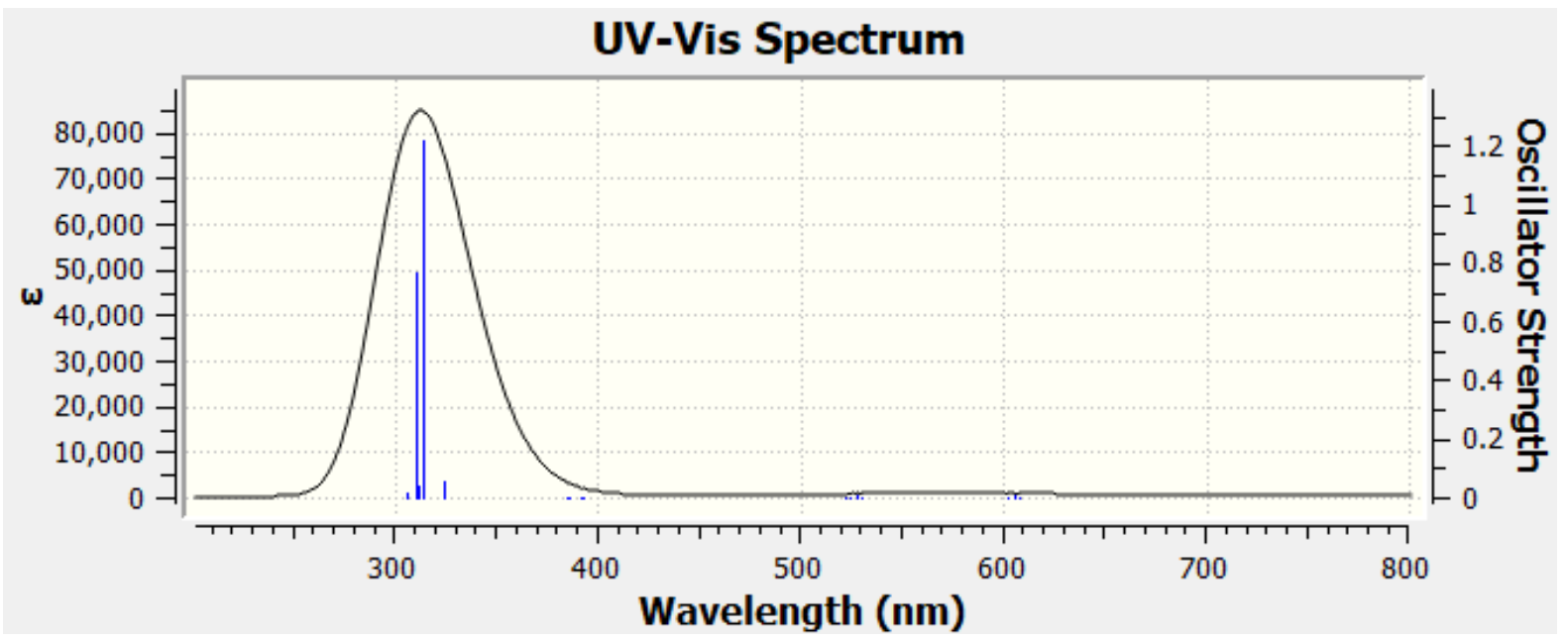

Figure S116: Calculated UV/Vis spectrum for compound 7cc 
Table S17: Assignment of transitions observed in the calculated UV/Vis spectra of compound 7cc

\begin{tabular}{|c|c|c|c|}
\hline $\begin{array}{c}\text { Wavelength } \\
(\mathrm{nm})\end{array}$ & $\begin{array}{l}\text { Oscillator } \\
\text { Strength } \\
\text { (f) }\end{array}$ & Assignment & $\begin{array}{l}\text { Excited } \\
\text { State }\end{array}$ \\
\hline 606 & 0.0046 & $\begin{array}{l}H-5 \rightarrow L+1(2.90 \%), H-5 \rightarrow L+6 \\
(7.58 \%), H-4 \rightarrow L+7(6.32 \%), H- \\
4 \rightarrow L+8(2.32 \%), H-3 \rightarrow L+1(2.40 \%), \\
H-3 \rightarrow L+2(13.64 \%), H-3 \rightarrow L+6 \\
(4.33 \%), H-2 \rightarrow L(3.96 \%), H-2 \rightarrow L+5 \\
(11.07 \%), H-1 \rightarrow L+2(3.49 \%), H- \\
1 \rightarrow L+8(4.66 \%), H \rightarrow L(4.18 \%), \\
H \rightarrow L+5(6.67 \%), H-1 \rightarrow L+7(4.45 \%)\end{array}$ & 3 \\
\hline 528 & 0.0049 & $\begin{array}{l}H-8 \rightarrow L+1(5.75 \%), H-8 \rightarrow L+6 \\
(15.19 \%), H-7 \rightarrow L(4.85 \%), H-7 \rightarrow L+5 \\
(13.72 \%), H-5 \rightarrow L+6(2.60 \%), H- \\
4 \rightarrow L+5(2.03 \%), H-4 \rightarrow L+7(2.15 \%) \\
H-3 \rightarrow L+2(18.76 \%), H-2 \rightarrow L+7 \\
(6.40 \%), H-2 \rightarrow L+8(2.32 \%), H- \\
1 \rightarrow L+6(3.40 \%), H \rightarrow L+5(3.24 \%)\end{array}$ & 6 \\
\hline 392 & 0.0032 & $\begin{array}{l}H-8 \rightarrow L+1(7.84 \%), H-8 \rightarrow L+6 \\
(15.45 \%), H-7 \rightarrow L(7.03 \%), H-7 \rightarrow L+5 \\
(14.57 \%), H-5 \rightarrow L+1(2.80 \%), H- \\
5 \rightarrow L+6(3.04 \%), H-4 \rightarrow L(2.24 \%), H- \\
4 \rightarrow L+5(3.86 \%), H-3 \rightarrow L+2(16.22 \%), \\
H-2 \rightarrow L+7(5.87 \%), H \rightarrow L(3.85 \%), \\
H \rightarrow L+5(2.94 \%)\end{array}$ & 10 \\
\hline 315 & 1.2168 & $\begin{array}{l}H-5 \rightarrow L+6(2.23 \%), H-4 \rightarrow L(2.36 \%) \\
H-1 \rightarrow L+1(2.38 \%), H-1 \rightarrow L+6 \\
(2.72 \%), H \rightarrow L(77.93 \%), H \rightarrow L+5 \\
(3.64 \%)\end{array}$ & 14 \\
\hline 310 & 0.7648 & $\begin{array}{l}\mathrm{H}-2 \rightarrow \mathrm{L}(7.65 \%), \mathrm{H}-1 \rightarrow \mathrm{L}+1 \text { (77.12\%), } \\
\mathrm{H} \rightarrow \mathrm{L}(5.02 \%)\end{array}$ & 16 \\
\hline
\end{tabular}




\section{References}

(1) Inkpen, M. S.; Du, S.; Driver, M.; Albrecht, T.; Long, N. J. Oxidative Purification of Halogenated Ferrocenes. Dalton Trans. 2013, 42 (8), 2813-2816.

(2) Wilson, L. E.; Hassenrück, C.; Winter, R. F.; White, A. J. P.; Albrecht, T.; Long, N. J. Functionalised Biferrocene Systems towards Molecular Electronics. Eur. J. Inorg. Chem. 2017, 2017 (2), 496504.

(3) Yuan, Y.; Yan, J. F.; Lin, D. Q.; Mao, B. W.; Yuan, Y. F. Ferrocene-Alkynyl Conjugated Molecular Wires: Synthesis, Characterization, and Conductance Properties. Chem. Eur. J. 2018, 24 (14), 3545-3555.

(4) Inkpen, M. S.; Albrecht, T.; Long, N. J. Branched Redox-Active Complexes for the Study of Novel Charge Transport Processes. Organometallics 2013, 32 (20), 6053-6060.

(5) Inkpen, M. S.; White, A. J. P.; Albrecht, T.; Long, N. J. Avoiding Problem Reactions at the Ferrocenyl-Alkyne Motif: A Convenient Synthesis of Model, Redox-Active Complexes for Molecular Electronics. Dalton Trans. 2014, 43 (41), 15287-15290.

(6) Hsung, R. P.; Chidsey, C. E. D.; Sita, L. R. Synthesis and Characterization of Unsymmetric Ferrocene-Terminated Phenylethynyl Oligomers $\mathrm{Cp}_{2} \mathrm{Fe}$-[C.Tplbond.C- $\left.\mathrm{C}_{6} \mathrm{H}_{4}\right] \mathrm{n}-\mathrm{X},(\mathrm{X}=\mathrm{SH}, \mathrm{SMe}$, SOMe, and $\mathrm{SO}_{2} \mathrm{Me}$ ). Organometallics 1995, 14 (10), 4808-4815.

(7) Swinehart, D. F. The Beer-Lambert Law. J. Chem. Educ. 1962, 39 (7), 333-335.

(8) Frisch, M. J.; Trucks, G. W.; Schlegel, H. B.; Scuseria, G. E.; Robb, M. A.; Cheeseman, J. R.; Scalmani, G.; Barone, V.; Petersson, G. A.; Nakatsuji, H.; Li, X.; Caricato, M.; Marenich, A. V.; Bloino, J.; Janesko, B. G.; Gomperts, R.; Mennucci, B.; Hratch, D. J. Gaussian 16, Revision C.01. Gaussian, Inc.: Wallingford CT 2016.

(9) Dennington, Roy; Keith, Todd A.; Millam, J. M. GaussView, Version 6. Semichem Inc.: Shawnee Mission, KS 2016. 